AUS DEM LEHRSTUHL

FÜR KLINISCHE CHEMIE, LABORATORIUMSMEDIZIN

UND TRANSFUSIONSMEDIZIN

DIREKTOR: PROF. DR. MED. GERD SCHMITZ

DER FAKULTÄT FÜR MEDIZIN

DER UNIVERSITÄT REGENSBURG

\title{
PLATELET-DERIVED EXTRACELLULAR VESICLES \\ IN PLATELETPHERESIS CONCENTRATES \\ AS A QUALITY CONTROL APPROACH
}

\author{
Dissertation \\ zur Erlangung des Doktorgrades \\ der Medizin \\ der \\ Fakultät für Medizin \\ der Universität Regensburg
}

vorgelegt von

Anne Black (geb. Dzikus) 

AUS DEM LEHRSTUHL

FÜR KLINISCHE CHEMIE, LABORATORIUMSMEDIZIN

UND TRANSFUSIONSMEDIZIN

DIREKTOR: PROF. DR. MED. GERD SCHMITZ

DER FAKULTÄT FÜR MEDIZIN

DER UNIVERSITÄT REGENSBURG

\title{
PLATELET-DERIVED EXTRACELLULAR VESICLES IN PLATELETPHERESIS CONCENTRATES AS A QUALITY CONTROL APPROACH
}

\author{
Dissertation \\ zur Erlangung des Doktorgrades \\ der Medizin \\ der \\ Fakultät für Medizin \\ der Universität Regensburg \\ vorgelegt von \\ Anne Black (geb. Dzikus)
}


Dekan:

1. Berichterstatter:

2. Berichterstatter:

Tag der mündlichen Prüfung:
Prof. Dr. Dr. Torsten E. Reichert

Prof. Dr. Gerd Schmitz

Prof. Dr. Ernst Holler

16.09.2014 
Table of contents

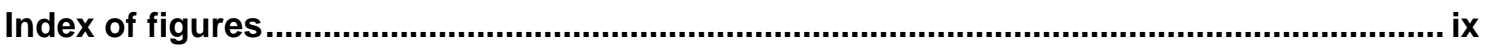

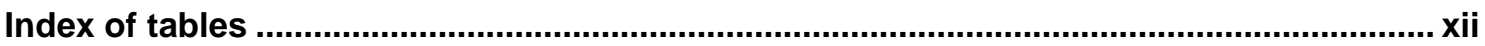

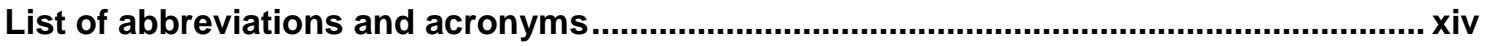

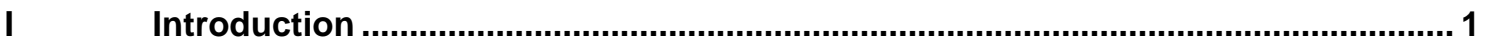

1.1 Platelet-derived extracellular vesicles in platelet storage lesion and platelet senescence $\quad 1$

$\begin{array}{lll}1.2 & \text { Plateletpheresis concentrate quality } & 7\end{array}$

$\begin{array}{lll}1.3 & \text { Extracellular vesicles in general } & 10\end{array}$

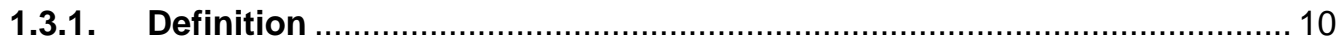

1.3.2. Function of extracellular vesicles ............................................................ 14

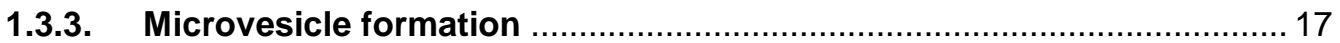

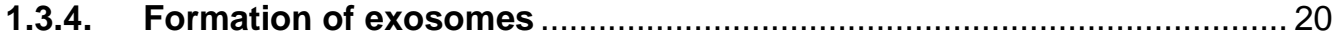

$1.4 \quad$ Platelet-derived extracellular vesicles 21

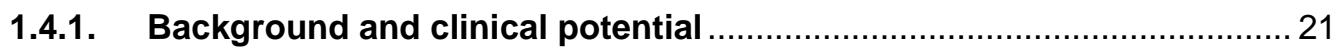

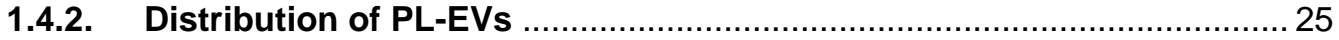

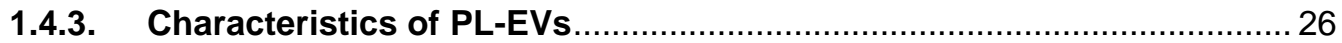

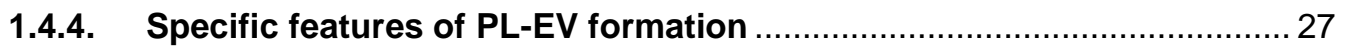

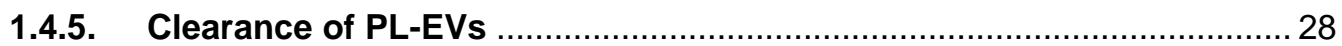

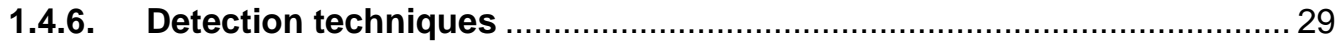

II Aims of the study

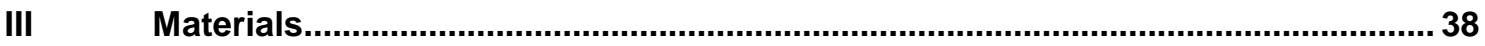

$\begin{array}{lll}3.1 & \text { Technical equipment } & 38\end{array}$

$\begin{array}{lll}3.2 & \text { Consumables } & 39\end{array}$

$\begin{array}{llr}3.3 & \text { Reagents } & 40\end{array}$

$\begin{array}{lll}3.4 & \text { Analytical Software } & 42\end{array}$

3.5 Preparation of solutions 43

3.5.1 TRAP-6

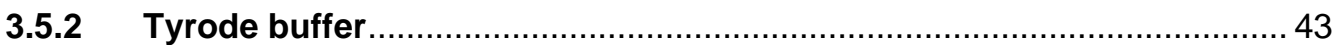


3.6 Plateletpheresis concentrates 43

$\begin{array}{lll}3.7 & \text { Red blood cell units } & 44\end{array}$

$\begin{array}{lll}\text { 3.8 } & \text { Blood samples of healthy donors } & 44\end{array}$

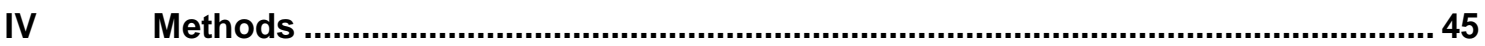

$\begin{array}{lll}4.1 & \text { Background } & 45\end{array}$

4.2 Flow chart of plateletpheresis and sampling for quality control 46

$\begin{array}{lll}\text { 4.3 Sampling of platelet concentrates } & 47\end{array}$

4.4 Sample preparation of platelet concentrates for measurement of platelet-derived and red blood cell-derived extracellular vesicles 50

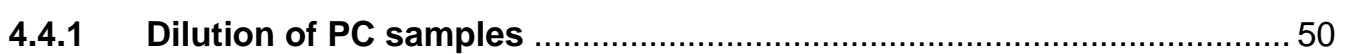

4.4.2 Platelet- and EV-containing plasma preparation................................ 50

4.4.3 Preparative isolation of EVs from plateletpheresis

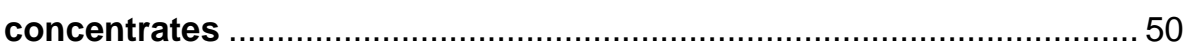

4.5 Sample preparation of red blood cell units for measurement of plateletderived and red blood cell-derived extracellular vesicles 50

$\begin{array}{lll}\text { 4.6 Flow cytometry } & 51\end{array}$

4.6.1 Principles and parameters

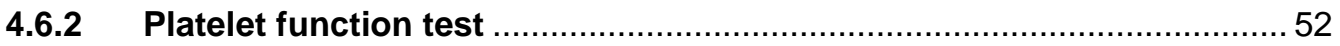

4.6.3 Platelet-derived extracellular vesicles ….......................................... 54

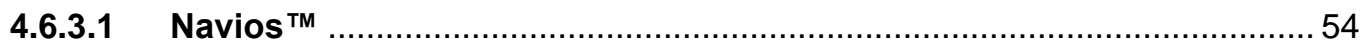

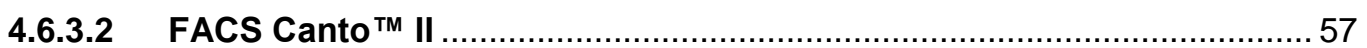

4.6.3.3 Apogee

$\begin{array}{lll}\text { 4.7 } & \text { Nanoparticle Tracking Analysis } & 60\end{array}$

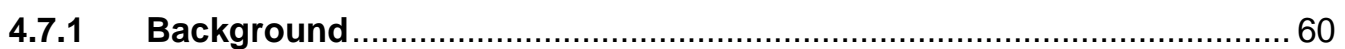

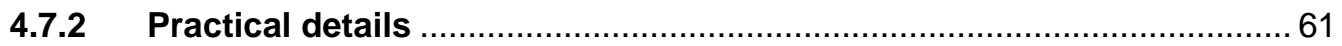

$\begin{array}{lll}4.8 & \text { Aggregometry } & 61\end{array}$

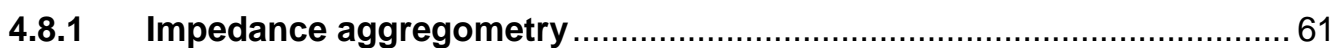

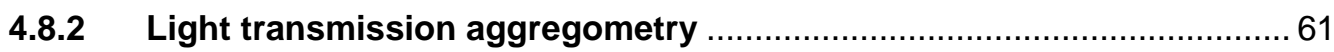

4.9 Total blood count and platelet count analysis 62

$\begin{array}{lll}4.10 & \text { Photometry } & 62\end{array}$ 
$\begin{array}{lll}4.11 & \text { Nephelometry } & 62\end{array}$

4.12 Enzym-Linked Immunosorbent Assay (ELISA) 63

4.13 Chemiluminescence immunoassay (CLIA) 63

$\begin{array}{ll}4.14 & \text { Coagulometry } 63\end{array}$

4.15 Chromogenic method 63

$\begin{array}{ll}4.16 & \text { Immunturbidometry }\end{array}$

$\begin{array}{ll}4.17 & \text { Potentiometric method }\end{array}$

$\begin{array}{lll}4.18 & \text { Statistical analysis } & 64\end{array}$

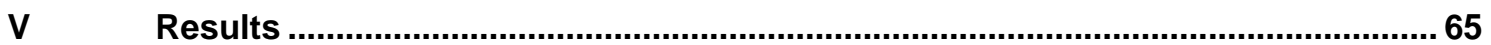

$\begin{array}{lll}5.1 & \text { Results of methodological issues } & 65\end{array}$

5.1.1 Validation of PL-EV measurement with standard flow

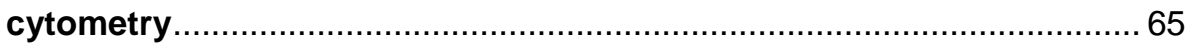

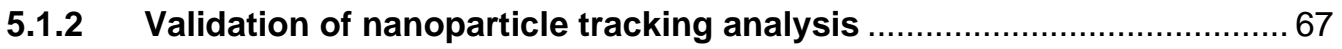

5.1.3 Analysis of plasma-vesicle-measurement by different flow cytometers and nanoparticle tracking system ..................................... 69

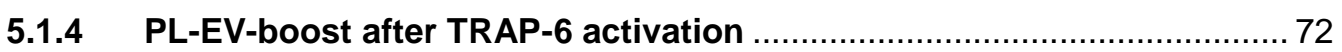

5.1.5 Rebound phenomenon, dependent on type of sampling and

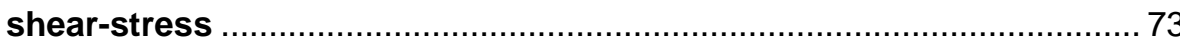

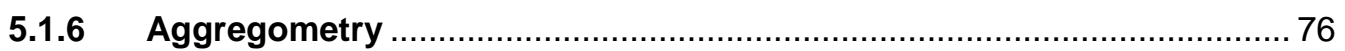

$\begin{array}{lll}5.2 & \text { Donor and apheresis specific values } & 77\end{array}$

$\begin{array}{lll}\text { 5.3 Platelet concentrate specific values } & 84\end{array}$

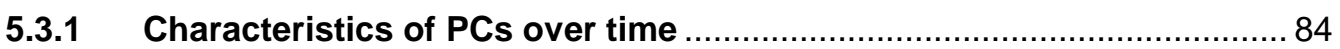

5.3.2 Platelet-derived extracellular vesicles and storage …......................... 85

5.3.3 Comparability of sample types (PC samples and tube samples) ........... 87

5.3.4 Effects of irradiation (Differences between non-irradiated

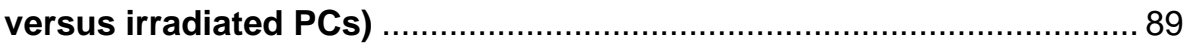

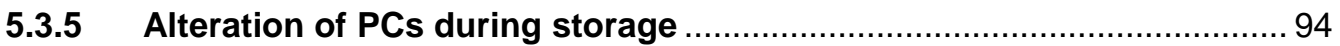

5.3.6 Linear regression analysis of platelet CD62P expression and PL-EV levels (sd-FCM) 100

5.3.7 Correlation between standard PL-EV analysis (sd-FCM) and the new systems (hs-FCM and NTA) 
5.3.8 Correlation between platelets CD62P and PL-EV analyses by hs-FCM and NTA

5.4 Extracellular vesicle composition in plateletpheresis concentrates and red blood cell units

VI Discussion 115

$\mathrm{V}$

Summary. 134

VIII Zusammenfassung 135

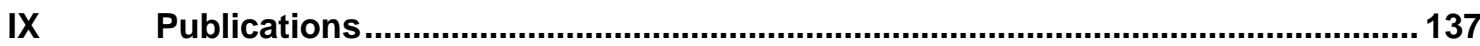

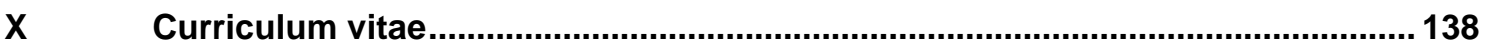

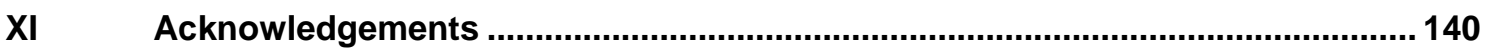

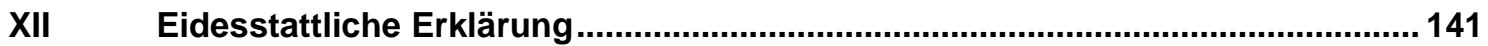

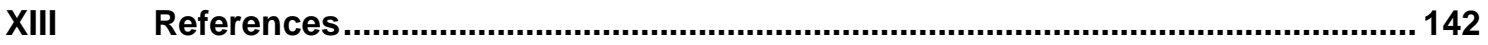




\section{Index of figures}

Figure 1-1: Summary of changes related to platelet storage lesion (PSL) and formation of platelet-derived extracellular vesicles (PL-EVs)

Figure I-2: Potential mechanisms behind the presence of platelet-derived extracellular vesicles (PL-EVs) in plateletpheresis concentrates (PCs)

Figure I-3: Scheme of eukaryotic cell forming microvesicles, exosomes and apoptotic bodies 12

Figure I-4: Summary of the main functions of extracellular vesicles (EVs) .............................. 14

Figure l-5: The formation of microvesicles from the cell membrane ...................................... 18

Figure II-1: Investigation of quality in plateletpheresis concentrates in vitro involves the analysis of separate concentrate components, i.e. plasma, platelets and platelet-derived extracellular vesicles (PL-EVs) 37

Figure IV-1: Workflow diagram for manufacturing and sampling of platelet concentrates $(P C)$ on several days after apheresis......................................................................................... 46

Figure IV-2: Flowchart of sample count $(\mathrm{n}=)$ from plateletpheresis concentrates...................... 46

Figure IV-3: Disconnection of the tube of plateletpheresis set after donation .......................... 48

Figure IV-4: Flexible tube filled with plateletpheresis product after disconnection from the apheresis set from a double donation ............................................................................. 48

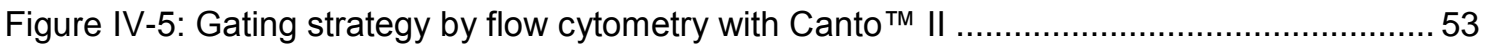

Figure IV-6: Absolute values of platelet events and Mean Fluorescence Intensity by analytical

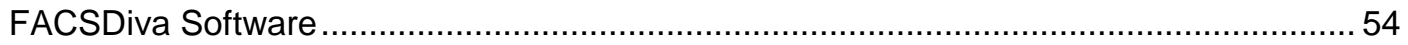

Figure IV-7: Changes of externalization of CD62P after activation with TRAP-6 by flow

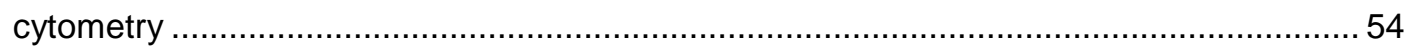

Figure IV-8: Calibration of instrument settings for EV-analysis with Navios ${ }^{\mathrm{TM}}$........................... 55

Figure IV-9: Gating strategy for EV analysis by flow cytometry with Navios ${ }^{\mathrm{TM}} \ldots \ldots \ldots \ldots \ldots \ldots \ldots \ldots . . . . . . . . . . . . .6$

Figure IV-10: Scatter plots of events for platelets and platelet-derived EVs by flow cytometry with Navios ${ }^{\mathrm{TM}}$ .56

Figure IV-11: Calibration of the instrument settings for EV-analysis with Canto ${ }^{\mathrm{TM}}$ II ..................5 57

Figure IV-12: Gating strategies for EV-analysis by flow cytometry with Canto ${ }^{\mathrm{TM}} \|$.................... 58

Figure IV-13: Platelet-derived EV analysis by flow cytometry with Canto ${ }^{\mathrm{TM}} \| \ldots \ldots \ldots \ldots \ldots \ldots \ldots \ldots . . . . . . . . . . . . .58$

Figure IV-14: Histogram of calibration of the instrument settings of Apogee A-50 Micro ...........59

Figure V-1: Validation of PL-EV measurement by standard flow cytometry ............................... 66 
Figure V-2: Linearity of the method for nanoparticle tracking analysis (NTA) with beads 67

Figure V-3: Linearity and accuracy of plasma vesicle size analysis (A) and recovery and accuracy of plasma vesicle concentration (B) by NTA .68

Figure V-4: Cumulative graph represents proof of size recovery for NTA with monodispers bead solutions. .68

Figure V-5: Accuracy of size measurements with mixed up polystyrene bead solutions 69

Figure V-6: Line chart of plasma vesicle size of plateletpheresis concentrates 71

Figure V-7: Inter-assay coefficients of variability .71

Figure V-8: Release of PL-EVs in response to TRAP-6 in fresh PCs on day 0 .72

Figure V-9: Rebound effect of platelet vesiculation and platelet function over time 74

Figure V-10: Differences in rebound phenomenon depending on shear stress .75

Figure V-11: Effects of PL-EVs on platelet aggregation 76

Figure V-12: Effect of single-needle versus double-needle apheresis system on CD62P 81

Figure V-13: Effect of apheresis instruments on PL-EV levels in PCs 81

Figure V-14: Box plots for platelet count values of two sample types of plateletpheresis quality control analysis .87

Figure V-15: Box plots for CD62P expression on platelets from PCs of two sample types....... 88

Figure V-16: Box plots of the interquartile range (IQR) for PL-EV raw data of different sample types ...... 89

Figure V-17: Box plots illustrating the effect of irradiation on platelet count .90

Figure V-18: Box plots illustrating the effect of irradiation on PL-EV levels measured by sd-FCM 90

Figure V-19: Effect of irradiation on the PL-EV/ PLT ratio in fresh PCs measured by hs-FCM.. 91 Figure V-20: Effect of irradiation on PL-EV and PLT count in PCs measured by hs-FCM ......... 92 Figure V-21: Effect of irradiation on the vesicle concentration in PCs measured by NTA .......... 93 Figure V-22: Effect of irradiation on size distribution of vesicles in PCs measured by NTA ....... 93 Figure V-23: Box plots demonstrating changes of platelet count over time. .94

Figure V-24: Box plots of different CD62P externalization on platelets of PCs over time (A) and after irradiation (B) .95

Figure V-25: Box plots of shedded PL-EVs in PCs over time measured by sd-FCM 96 
Figure V-26: Box plots of differences in PL-EVs depending on irradiation measured by sd-FCM

Figure V-27: Scatter plot of the correlation between PL-EVs of fresh versus expired PCs

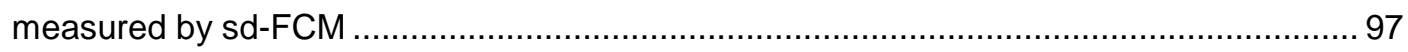

Figure V-28: Box plots of PL-EV ratio of irradiated PCs over time measured by hs-FCM..........98

Figure V-29: Box plots of plasma vesicles concentration of irradiated PCs over time measured by NTA 98

Figure V-30: Box plots of IPF of fresh and stored PCs measured by automated hematology analyzer 99

Figure V-31: Box plots of $\mathrm{pH}$ values of PCs over time and the effects of irradiation .99

Figure V-32: Scatter plot distribution of PL-EVs in correlation/ linear regression to CD62P externalization. 101

Figure V-33: Comparison of sd-FCM and hs-FCM PL-EV analysis on day 0. 102

Figure V-34: PL-EV analysis by hs-FCM (A) and sd-FCM (B) 103

Figure V-35: Evaluation of plasma vesicles from PCs by NTA 104

Figure V-36: Correlation between CD62P expression and the PL-EV-ratio by hs-FCM 106

Figure V-37: Correlation between CD62P (after stimulation with TRAP-6) and PL-EV subpopulations in fresh non-irradiated PCs measured by hs-FCM.

Figure V-38: Correlation between CD62P (after TRAP-6 stimulation) and PL-EV subpopulations in fresh irradiated PCs measured by hs-FCM 109

Figure V-39: Correlation between CD62P (after stimulation with TRAP-6) and PL-EV subpopulations in senescent irradiated PCs measured by hs-FCM 109

Figure V-40: Composition of cell-derived vesicles in PCs 111

Figure V-41: Pie chart of the cell-derived EV percentage in red blood cell units...... 112

Figure V-42: Platelet-derived EVs and erythrocyte-derived EVs from red blood cell units 113

Figure V-43: Plasma and cell-derived vesicles from red blood cell units measured by NTA ... 114 


\section{Index of tables}

Table I-1: Potential mechanisms of PL-EV formation during collection and storage .................... 5

Table I-2: In vitro tests of platelet concentrate quality monitoring ............................................ 8

Table I-3: Parameters and criteria of quality control in manufacturing of plateletpheresis

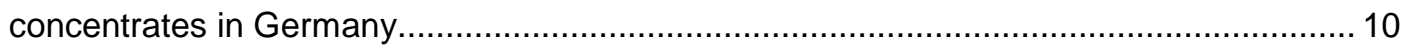

Table I-4: Overview of three subgroups of extracellular vesicles............................................ 13

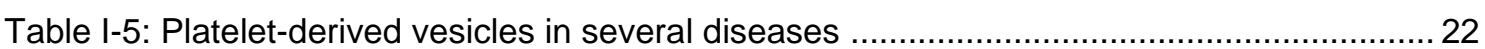

Table I-6: This overview shows common and variable glycoprotein (GP) receptors or activation markers of platelet-derived and megakaryocyte-derived extracellular vesicles (EVs) ....... 27

Table I-7: Pros and cons of detection techniques for measurement of extracellular vesicles .... 30

Table 1-8: Designation of PL-EV concentrations from plasma samples of healthy individuals by flow cytometry as reported in the literature.

Table I-9: PL-EV concentration of platelet concentrate samples measured by flow cytometry as

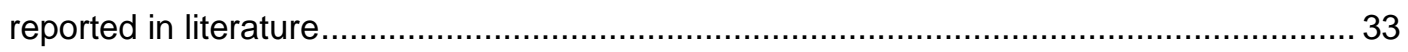

Table I-10: Bead and vesicle characteristics for detection by light scatter ................................. 34

Table IV-1: Summary of count $(n=)$ from plateletpheresis concentrate $(P C)$ samples and tube samples for analysis on day 0 and day 5 (red numbers belong to the irradiated samples) 49

Table IV-2: Preparation of red blood cell units for analysis of extracellular vesicles by ultracentrifugation and density gradient centrifugation .................................................... 51

Table IV-3: Scheme of immunostaining for analyzing PLT function test ................................... 53

Table V-1: Vesicle quantification of plateletpheresis concentrates (PCs) by NTA ..................... 70

Table V-2: Characteristics of donors and of the apheresis process ...................................... 77

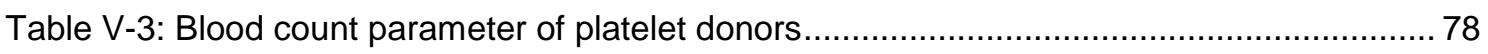

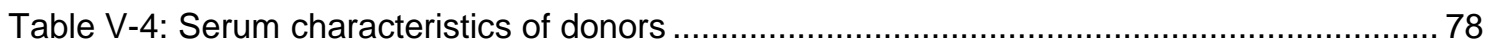

Table V-5: Relevant correlation of donor-specific, clinical or laboratory parameters to PC quality 80

Table V-6: Relevant donor specific parameters in correlation to one another ........................... 83

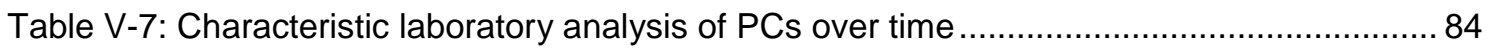

Table V-8: PL-EV and plasma vesicle values in PCs analyzed by flow cytometry and NTA ...... 86

Table V-9: PL-EV analysis by sd-FCM versus hs-FCM or NTA............................................. 105 
Table V-10: Correlation between platelet CD62P expression after TRAP-6 determined by hsFCM and by NTA.

Table VI-1: Comparison of PL-EV concentration from PCs on day 0 or day 1 reported in recent literature 


\section{List of abbreviations and acronyms}

\begin{tabular}{|c|c|}
\hline ADP & adenosine diphosphate \\
\hline AFM & atomic force microscopy \\
\hline Apo A-I & apolipoprotein A-I \\
\hline Apo B100 & apolipoprotein B100 \\
\hline Apo B48 & apolipoprotein B48 \\
\hline Apo C-I & apolipoprotein C-I \\
\hline Apo $E$ & apolipoprotein E \\
\hline Apo J & apolipoprotein $\mathrm{J}$ (clusterin) \\
\hline approx. & approximately \\
\hline ATIII & antithrombin III \\
\hline ATP & adenosine triphosphate \\
\hline BMI & body mass index \\
\hline BMP & bis-(monoacyl)-glycerophosphate \\
\hline C3c & complement factor C3 \\
\hline $\mathrm{cc}$ & correlation coefficient \\
\hline CD & cluster of differentiation \\
\hline CD40L & CD40 ligand, or CD154 \\
\hline CD41 & glycoprotein (GP) Ilb \\
\hline $\mathrm{CD} 42 \mathrm{~b}$ & glycoprotein (GP) Ib \\
\hline CD61 & glycoprotein (GP) IIla \\
\hline CD62P & p-selectin \\
\hline CD63 & a member of the tetraspanin superfamily of integralmembrane proteins \\
\hline CD95L & CD95 ligand, FASL, galectin 9 \\
\hline $\mathrm{CHOL}$ & cholesterol \\
\hline CR1 & complement receptor 1 \\
\hline CRACM1 & calcium release-activated calcium channel protein/modulator 1 \\
\hline CRP & C-reactive protein \\
\hline CV & coefficient of variation \\
\hline C1-INH & C1 esterase inhibitor \\
\hline DC & dendritic cells \\
\hline DIGE & differential in-gel electrophoresis \\
\hline
\end{tabular}




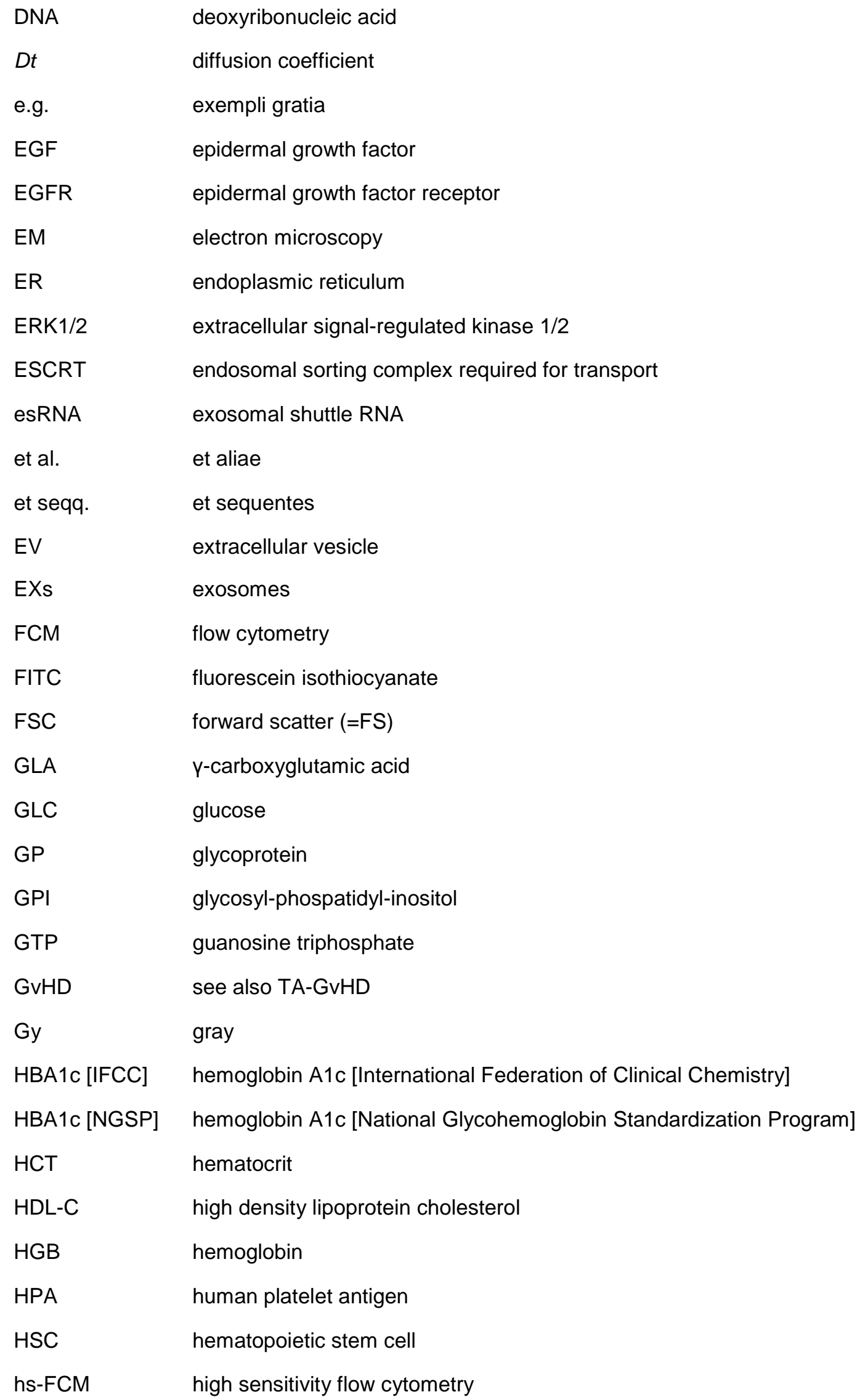




\begin{tabular}{|c|c|}
\hline HSP & heat shock protein \\
\hline i.e. & id est \\
\hline IGF-1 & insulin-like growth factor 1 \\
\hline IGFBP3 & insulin-like growth factor binding protein 3 \\
\hline ILV & intraluminal vesicle \\
\hline IPF & immature platelet fraction \\
\hline ITP & idiopathic thrombocytopenic purpura \\
\hline$K_{B}$ & Boltzmann's constant \\
\hline LALS & large angle light scatter $\approx S S C$ \\
\hline LAMP-1 & lysosome-associated membrane glycoprotein-1 \\
\hline LDL-C & low-density lipoprotein cholesterol \\
\hline L-EV & leukocyte-derived extracellular vesicle \\
\hline $\operatorname{Lp}(a)$ & lipoprotein (a) \\
\hline LPA & lysophosphatidic acid \\
\hline LPS & lipopolysaccharide \\
\hline MFG-E8 & milk fat globule - epidermal growth factor - 8 \\
\hline MFI & mean fluorescent intensity \\
\hline $\mathrm{MHC}$ & major histocompatibility complex \\
\hline $\min$ & minutes \\
\hline miRNA & microRNA \\
\hline MK & megakaryocyte \\
\hline moAb & monoclonal antibody \\
\hline MODS & multiple organ dysfunction syndrome \\
\hline MP & microparticle \\
\hline MPV & mean platelet volume \\
\hline mRNA & messenger RNA \\
\hline MV & microvesicle \\
\hline MVB & multivesicular body \\
\hline MVE & multivesicular endosomes \\
\hline $\mathrm{mW}$ & milliwatt \\
\hline NTA & nanoparticle tracking analysis \\
\hline Orai1 & see also CRACM1 \\
\hline
\end{tabular}


P2X ${ }_{7}$ receptor purinergic receptor $\mathrm{P} 2 \mathrm{X}$, ligand-gated ion channel 7

PAI-1 plasminogen activator inhibitor-1

PBS

PBS-Dulbecco, w/o Ca ${ }^{2+} / \mathrm{Mg}^{2+}$ Buffer

PC

platelet(pheresis) concentrate

PC7

phycoerythrin cyanin 7

PDGF

platelet-derived growth factor

PDW

platelet distribution width

PE

phycoerythrin

PF3

platelet factor 3

PKC

protein kinase $\mathrm{C}$

PLT

platelet

PL-EV

platelet-derived extracellular vesicle

PMP

platelet microparticles

$\mathrm{PNH}$

paroxysmal nocturnal hemoglobinuria

PPI

particles per image

PS

phosphatidylserine

PSD

platelet storage defect

PSGL-1

p-selectin glycoprotein ligand-1

PSL

platelet storage lesion

$\mathrm{P} 2 \mathrm{X}_{1}$

purinergic receptor $\mathrm{P} 2 \mathrm{X}$, ligand-gated ion channel 1

$\mathrm{P} 2 \mathrm{X}_{7}$

purinergic receptor P2X, ligand-gated ion channel 7

$\mathrm{Rab}$

Ras-related in brain

Rap1

Ras-related protein 1

$\mathrm{RBC}$

red blood cell

RBC-EV

RBC-derived vesicle

RDW-CV

red cell distribution width as coefficient of variation from the mean red cell size

RDW-SD red cell distribution width as standard deviation from the mean red cell size

RES reticuloendothelial system

$r_{h} \quad$ hydrodynamic radius

$\mathrm{RI} \quad$ refractive index $(\eta)$

RNA ribonucleic acid 


\begin{tabular}{|c|c|}
\hline ROCK & Rho-associated coiled-coil-containing protein kinase \\
\hline $\mathrm{RR}$ & blood pressure according to Riva-Rocci \\
\hline SALS & small angle light scatter $\approx \mathrm{FSC}$ \\
\hline sd-FCM & standard flow cytometry \\
\hline sec & seconds \\
\hline SERCAs & sarco/ endoplasmic reticulum $\mathrm{Ca}^{2+}$-ATPases \\
\hline SFLLRN & ser-phen-leu-leu-arg-asn \\
\hline SNARE & soluble $\mathrm{N}$-ethylmaleimide-sensitive-factor attachment receptor \\
\hline soc & store-operated calcium \\
\hline SOCE & store-operated calcium entry \\
\hline SSC & side scatter (=SS) \\
\hline STIM1 & stromal-interacting molecule 1 \\
\hline$T$ & temperature \\
\hline TA-GvHD & transfusion associated graft-versus-host disease \\
\hline TCTP & translation controlled tumor protein \\
\hline TF & tissue factor \\
\hline TFPI & tissue factor pathway inhibitor \\
\hline TFG- $\beta 1$ & transforming growth factor $\beta 1$ \\
\hline TLR4 & toll-like receptor 4 \\
\hline TNF- $\alpha$ & tumor necrosis factor $\alpha$ \\
\hline TRAP-6 & thrombin receptor activating peptide 6 \\
\hline TRF & transferrin receptor \\
\hline TRIG & triglyceride \\
\hline TSG101 & tumor susceptibility gene 101 \\
\hline $\mathrm{TXA}_{2}$ & thromboxane A2 \\
\hline UC & ultracentrifuge \\
\hline V & volt \\
\hline VEGF & vascular endothelial growth factor \\
\hline VLDL-C & very low density lipoprotein cholesterol \\
\hline VSMC & vascular smooth muscle cells \\
\hline vWF & von Willebrand factor \\
\hline vWfr & von Willebrand factor receptor \\
\hline
\end{tabular}


$\eta$

$\pi$

${ }^{\circ} \mathrm{C}$ solvent viscosity

$\mathrm{Pi}$

degree centigrade 


\section{Introduction}

\subsection{Platelet-derived extracellular vesicles in platelet storage lesion and platelet senescence}

Anucleated platelets are small $(0.5-1 \mu \mathrm{m})$, discoid blood cells with a lifespan up to 10 days in the human circulatory system $(1,2)$. They play an essential role in primary hemostasis and are filled with $\alpha$-granules, dense granules and lysosomes (3). Intracellular $\alpha$-granules are surrounded by membranes which contain proteins, chemokines, growth factors and immune mediators. Immune mediators constitute a large group of active molecules which includes, among the others, glycoprotein (GP) Ilb-Illa (integrin allb33), p-selectin, factor V (FV), factor IX (FIX), protein $S$, tissue factor (TF), plasminogen, plasminogen activator inhibitor 1 (PAI-1), fibrinogen, von Willebrand factor (vWF), thrombospondin, growth factors (epidermal, insulin-like, vascular endothelium, fibroblast, platelet-derived), complement $\mathrm{C} 3$, complement $\mathrm{C} 4, \mathrm{C} 1$ inhibitor, $\beta 1 \mathrm{H}$ globulin (factor $\mathrm{H}$ ) and immunoglobulin $\mathrm{G}$ (IgG). Dense granules contain $\mathrm{Ca}^{2+}$, $\mathrm{Mg}^{2+}$ and $\mathrm{K}^{+}$, polyphosphate, pyrophosphate, serotonin, histamine and nucleotides (e.g. ADP and ATP). Platelet lysosomes are filled with enzymes degrading proteins (e.g. cathepsin, elastase and collagenase), carbohydrates (e.g. glucosidase, fucosidase and galactosidase) and lipids (e.g. acid phosphatase).

Additionally to granules and cytoskeletal components, platelets also contain other organelles such as mitochondria and the dense tubular system which is analogous to the endoplasmic reticulum connected to the open canalicular system and glycogen stores.

Circulating platelets undergo senescence prior to clearance; the cells are not capable of division, they are metabolically active, though. Ex-vivo resting platelets undergo platelet storage lesion and they are subjected to either activation-mediated death or to storage-induced death.

The storage "phenotype" of platelets, which translates to cell death, may also be responsible for necrotic or apoptotic changes (1), as well as for activation (2).

Platelet storage lesion (PSL) or platelet storage defect (PSD) comprise platelets which lost their typical functional characteristics. This means loss of membrane integrity, $p$-selectin release, shedding of surface proteins (receptors), diminished mitochondrial membrane potential, increase of the intracellular calcium level with a secondary activation of proteases (caspases), externalization of phosphatidylserine (PS) and secretion of platelet-derived extracellular vesicles (PL-EVs) (2), see Figure I-1. The presence of PSL justifiably corresponds to the reductions of in vivo survival and haemostatic activity after transfusion (4). Changes in PSL platelets are influenced by alterations during collection, processing and storage in platelet concentrates (PCs) (5). 


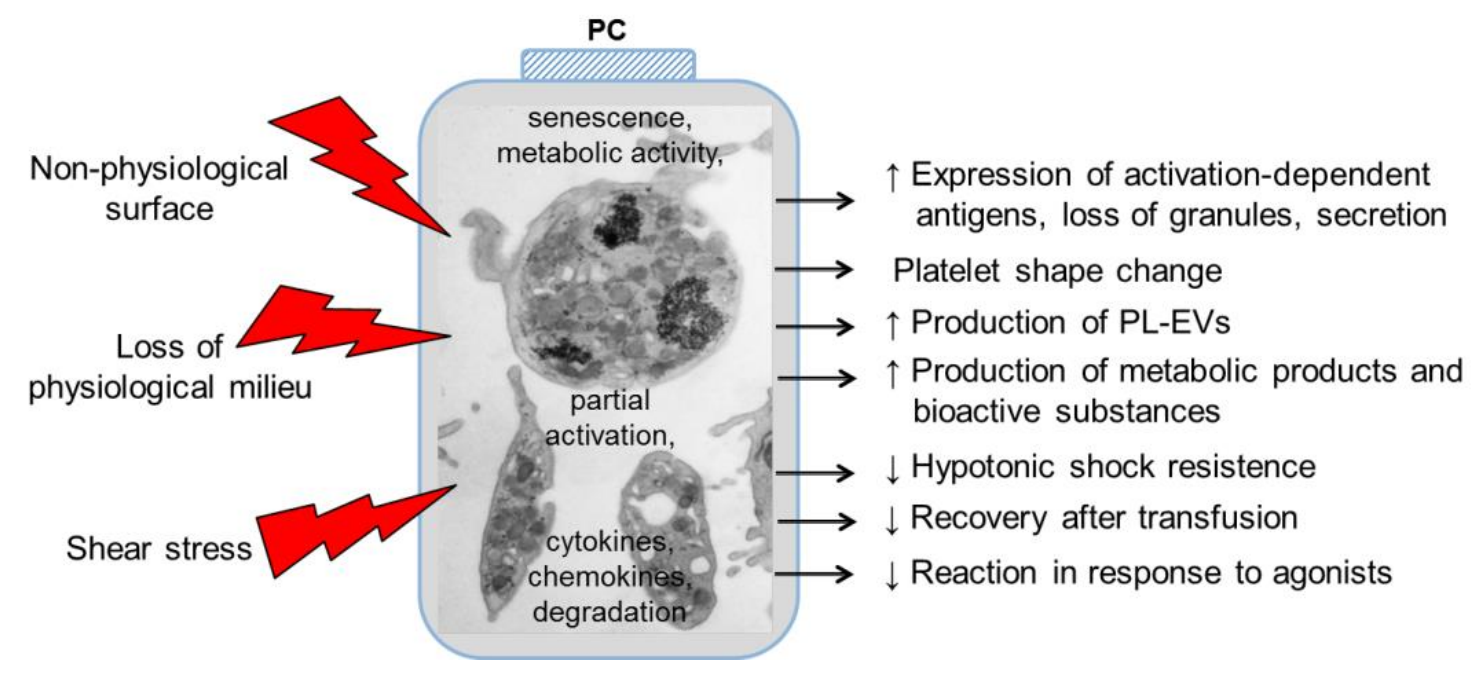

Figure 1-1: Summary of changes related to platelet storage lesion (PSL) and formation of platelet-derived extracellular vesicles (PL-EVs)

The figure demonstrates the effects of storage conditions ex vivo (left) and the modulation of platelet morphology, physiology and function (right).

Storage of platelet concentrates is performed under continuous agitation under standard blood banking conditions at $22^{\circ} \mathrm{C} \pm 2^{\circ} \mathrm{C}$ for 5 days, including the day of apheresis. Agitation is carried out in bags with oxygen exchange in order to maintain the aerobic adenosine triphosphate (ATP) formation. Obviously, there are several factors which influence PSL such as temperature, storage duration, form and intensity of agitation, volume of suspending plasma, permeability of the membranes of a storage container and a leukodepletion technique (5). Exemplarily, storage carried out almost at physiological temperatures $\left(37^{\circ} \mathrm{C}\right)$ improves the viability of platelets due to a decrease of a rapid ATP turnover and a lower metabolic activity of stored platelets $(5,6)$. Additionally, a $16 \%$-release of $\alpha$-granules within seven days of storage was described and the release was independent from different rotators used. This leads to a loss of functional proteins, essential for the adequate coagulation response after transfusion in vivo (7). To control the effects of these factors during storage of PCs, several techniques were used and incorporated into the quality control process (explained in detail in chapter 1.2, page 7).

PL-EVs were described first in 1967 as a coagulable "platelet dust" (8). They are also connected with the term of platelet factor 3 (PF3) activity and its role in coagulation (9). Platelet vesiculation led back to the observation of a 10-fold rise in the PF3 activity during platelet storage (10). The raise in PF3 activity is accompanied by a stable platelet count. Nearly three decades ago, an active PF3 was regarded as a part of PSL in a process of platelet activation and damage (11). The presence of PL-EVs or platelet-derived microvesicles (PL-MVs) in stored PCs has been well demonstrated. Already in 1986/87, Solberg et al. recognized it and found a correlation between the presence of PL-MVs and PF3 activity $(12,13)$. Formation of plateletderived vesicles particularly elevates in PCs exhibiting high $\mathrm{pH}$, associated with $\mathrm{LDH}$ release. 
In 1988, George et al. showed rotation dependency on PL-MV level over 7 days storage as a shear stress model. Inappropriate shear stress and/ or activation of platelets during storage result in elevated vesicle levels (7). During a study on supplementation of platelets with activation inhibitors that protect platelets from damage, the group of Bode demonstrated in 1991 that solely partial inhibition of vesiculation occurred $(40 \%)$, resulting in a hypothesis that not only activated platelets shed MVs. However, MVs seemed to appear already at physiological concentrations in matured platelets (14). The group of Bode delineated two populations of microparticles (MPs): one population corresponding to fluorescent beads smaller than $0.5 \mu \mathrm{m}$ and another one corresponding to fluorescent beads, larger than $0.5 \mu \mathrm{m}$.

Nowadays, it is well known that strongly procoagulant PL-MVs or PL-EVs released from membranes of intact platelets are present in PCs but there are many questions in platelet transfusion medicine which still remain unanswered.

Various functions of PL-MVs in PCs can be ascribed to four pathologic conditions (Figure I-2). The first condition is related to mechanical injury and shear stress during collection and processing. It is not distinguishable to which extent PL-MV components are released from donor platelets in vivo and "collected" in the suspended donor plasma as compared to the release via the non-physiological shear force of the apheresis systems. Rank et al. isolated annexin $\mathrm{V}$ positive microvesicles which reached the concentrates and the group compared the vesicle content of PCs to the MV content in the donor plasma (15). PL-MVs in PCs (93\% of all MVs) differed by 39\% from the PL-MV amount obtained from donor plasma (1.6 to 1), suggesting that plateletpheresis concentrates contain more PL-MVs than the donor's pre-donation samples and they seem to be constant in PL-MV enrichment during storage over 5 days. Rank et al. assumed that the most abundant amount of PL-MVs in PCs results from the collection process and not from the donor plasma. PL-MVs originate to a lesser extent from activated platelets ( $p$ selectin positive PL-MVs in 4.8\%) rather than from resting platelets with stable annexin $\mathrm{V}$ positive MVs. However, the group of Rank found increased degranulation of p-selectin in PLMVs dependent on the storage time. Furthermore, the results indicated that CD63 positive platelet-derived exosomes (PL-EXs) are present in isolated MVs. Additionally, exosomes showed a significant increase on the fifth day of storage thus representing a minor fraction (2.6\%) in comparison to the dominant larger PL-MVs (15). 


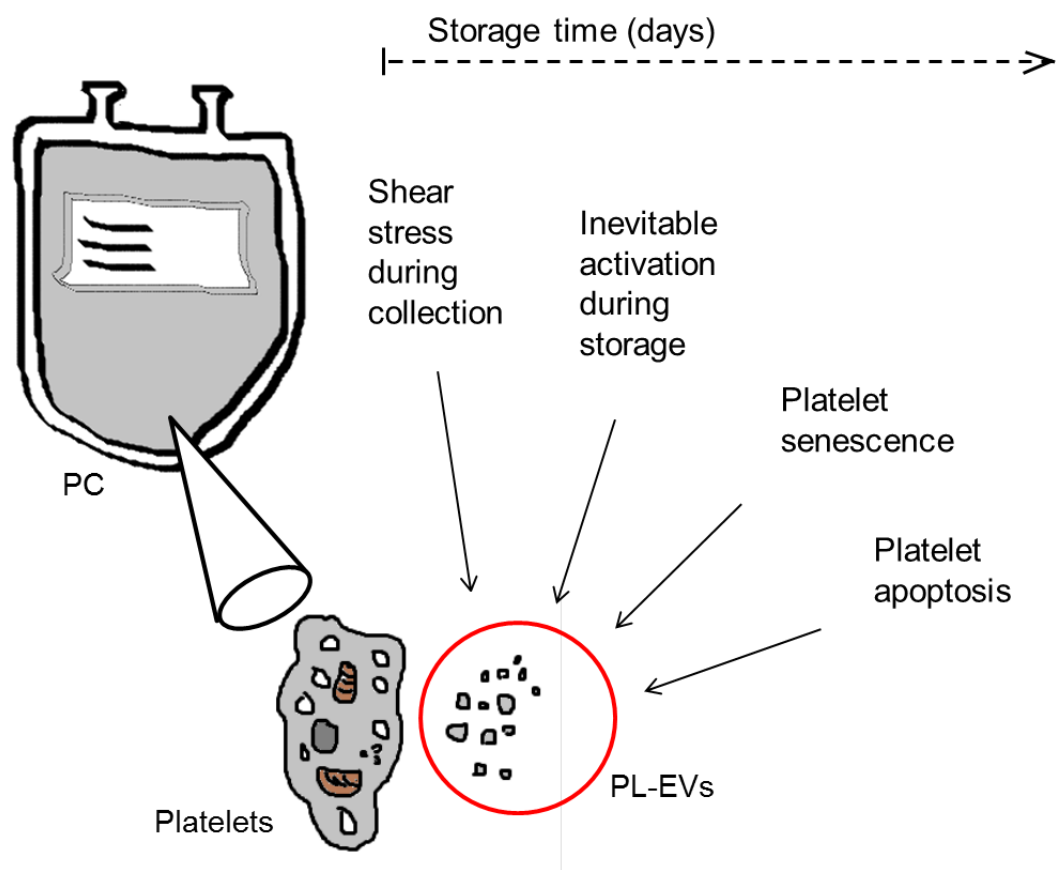

Figure I-2: Potential mechanisms behind the presence of platelet-derived extracellular vesicles (PL-EVs) in plateletpheresis concentrates (PCs)

Sloand et al. observed that the application of different preparation techniques (e.g. apheresis versus whole blood-derived PCs) and different anticoagulants (e.g. ACD versus CPDA-1) result in different amounts of PL-MVs (16). These findings support the hypothesis that the abundant amount of PL-MV in PCs is dependent on the conditions of collection and not necessarily on the donor plasma at the time of apheresis. However, at this point it cannot be excluded that there are donors, who have more sensitive platelet membranes and therefore generate more PL-MVs during plateletpheresis.

In addition, activation of stored platelets due to their contact with the plastic surface of a storage container takes place in the vesiculation fraction of PCs. Reduction of the surface-to-volume ratio $(\mathrm{S} / \mathrm{V})$ in $\mathrm{PCs}$ resulted in a lower $\mathrm{LDH}$ release and it significantly correlated to the total MP count, suggesting that the cytoplasmic content of all vesicles is regulated by the vesiculation of platelets (14). With the decrease in the adhesion of the platelets to the surface of the storage container, fewer platelets become activated thus being more prone to form platelet-derived vesicles.

Since 1997, various mechanisms have already been observed which "lead to microvesiculation of platelets during storage" (17), see Table I-1. Seghatchian et al. described available methods for quantification and characterization of PL-MVs. Nowadays, these methods overlap such evaluation approaches as flow cytometric analysis of specific markers (GPIb, GPIlb/llla) using monoclonal antibodies (moAbs) or analysis of PS exposure by annexin V labeling. Although, since then flow cytometry has been the favored method, the comparison of standard flow 
cytometers with advanced new high-resolution flow cytometers indicates more possibilities in detection of vesicles as far as their lower size and higher sensitivity are concerned.

Table I-1: Potential mechanisms of PL-EV formation during collection and storage

Mechanical disruption and excessive shear stress, e.g. passage through low gauge needles or leukocyte filters, high $g$ force, rigorous agitation

Activation by platelet aggregation agonists, e.g. ADP, thrombin, during collection and storage

Activation and secretion of platelets caused by poor handling and prolonged exposure to low temperatures $\left(4^{\circ} \mathrm{C}\right)$ and high $\mathrm{pH}>7.6$

Lysis of platelets caused by freezing-thawing, lyophilization and rehydration; prolonged exposure to low $\mathrm{pH}<6.2$

Exposure to activated complement components, leukocyte- and platelet-derived cytokines and enzymes, e.g. calpain, histamine

(Table adjusted to (17))

Senescence and apoptosis of platelets in PCs stored for five days could induce increased vesicle formation. With the purpose of explaining to which extent PL-MVs or PL-EXs originate from senescent or apoptotic platelets, it is essential to better define the "phenotype" of these vesicles compared to the vesicles from activated platelets. However, no evidence has been yet found to prove any principal differences between these vesicle groups. On the other hand, understanding of the process of vesicle formation during senescence and/ or apoptosis in PCs is indispensable and needs reinvestigation with the help of currently available more sophisticated methods. Though, despite of the fact that mechanisms of PSL and senescence may overlap, PSL and the apoptotic program are two distinct processes, even if some authors use the names of PSL and senescence interchangeably $(1,18)$.

As aforementioned, when inhibitors applied only, the reduction of activation-dependent generation of PL-EVs in stored platelets averages approximately $40 \%$. Nevertheless, significantly lower PL-EV levels within the storage time of 9 days versus control (14) suggest that remaining PL-EVs originate from resting/ senescent platelets. "In vitro experiments demonstrate that platelet apoptosis can be induced by calcium ionophores, other platelet agonists [during] storage at room temperature under blood banking conditions" (5). It is evident that platelets undergo apoptosis at $37^{\circ} \mathrm{C}$, accompanied by a loss of platelet viability and a gradual rise in caspase- 3 and caspase-9 activities (19). The inhibition of platelet apoptosis by 
cell-permeable caspase-inhibitors could improve the viability of platelets but this approach turned out to be ineffective in this study. Under this condition, gelsolin, a caspase- 3 substrate affected in apoptosis, was cleaved as caspase- 3 activity rose. This process, also observed by Thiele et al., became acknowledged as a cytosolic protein biomarker of apoptosis of platelets subjected to 9-day storage (20). Whereas $97 \%$ of cytosolic proteins remained unchanged, gelsolin and septin 2 belong to the group of $3 \%$ of proteins which undergo changes as analyzed by differential gel electrophoresis (DIGE) and mass spectrometry.

Thiele et al. also found proteins involved in the early storage lesion, e.g. the focal adhesion signaling integrin $\alpha$ llb $\beta 3(21)$, which plays a role as a proteomic biomarker of platelet quality. The investigation of changes of cytosolic proteins during storage, especially of the proteins connected to signaling pathways underlying storage lesion development (22), may at least partially correspond to activation, senescence and apoptosis of platelets and the underlying vesiculation.

PS exposure, detectable by annexin $\mathrm{V}$ binding and release of $p$-selectin provide evidence for an effect of apoptosis on platelet viability.

Although platelet mitochondria maintain their transmembrane potential $(\Delta \psi(m))$ upon storage over 7 days, which does not correlate to the downstream protein markers, selected stressors (i.e. calcium ionophore stimulation) lead to higher degrees of mitochondrial depolarization (23). The preparation techniques also affect the $\Delta \psi(\mathrm{m})$ of platelets and the percentage of platelets with depolarized $\Delta \psi(\mathrm{m})$ significantly increases in buffy-coat PCs as compared with PCs produced by apheresis (24).

In addition to the observed mitochondrial changes, the group of Albanyan showed growing values, both percentage and absolute, of the immature platelet fraction (IPF) as determined by Sysmex XE-2100 in PCs over 7-day storage (25). Simultaneously, the platelet count remained stable, whereas mean platelet volume (MPV) and platelet distribution width (PDW) exhibited a small increase. The fluorescent dye Thiazole Orange, used for detection of immature platelets with residuals of nucleic acid shows non-specific binding to basic proteins and anionic phospholipids which appear on the platelet surface during senescence. Dense granular or other platelet structures may become also unspecifically dyed because of their nucleotides or messenger RNA (mRNA) which affect the elevation of IPF levels. Thus far, no investigation has confirmed the presence of mRNA or micro RNA (miRNA) in PL-EVs. Yet, their presence is very likely to the expression of miRNA, detected in human peripheral blood vesicles (26-28) and most vesicles in circulating blood originate from platelets (or megakaryocytes, see chapter 1.4.2, page 25). It cannot be excluded that platelet-derived vesicles, which contain mRNA or miRNA, contribute to unspecific staining.

Gamma irradiation of platelet concentrates prevents transfusion-associated graft-versus-host disease (TA-GvHD) and may lead to an increase of vesiculation. Upon application of the required irradiation dose of 25 Gray (Gy), as assessed by Food and Drug Administration (FDA), 
no differences were found between irradiated and non-irradiated PCs, including swirling, hypotonic shock response (HSR), the percentage of GPlb-expressing cells and $\mathrm{pH}$ during 7 days of storage (29). On the other hand, development of elasticity points to a slower progression after storage in the irradiated group leading to the assumption, that vesiculation increases under the condition of the loss of membrane elasticity. Gamma irradiation applied on the first day of storage causes pronounced proteome changes, responsible for specific catalytic activities and/ or protein/ nucleic acid binding capacity, involved in the platelet storage lesion in PCs (30).

\section{$1.2 \quad$ Plateletpheresis concentrate quality}

It is essential to carry out laboratory tests in order to guarantee consistent and uniform quality of plateletpheresis concentrates. However, there is no single laboratory test which would reflect potential hemostatic effects of platelets in vivo and there is no established benchmark which can be used to translate the measured loss of platelet function to an appropriate graduation of PC quality (17). Many applicable tests were validated for identifying PSL of platelets. Nevertheless, there are established standards for platelet concentrate quality within quality control (QC) programs to detect problems during collection, processing or storage (5) according to the corresponding guidelines. There are currently several protocols for quality control used; the most popular are as follows: (i) the AABB (American Association of Blood Banks) technical manual (31) for the United States, (ii) the Guide to preparation, use and quality assurance of blood components (32) for Europe and (iii) the German guidelines for production and application of blood and blood components (33).

These guidelines recommend determination of platelet count (number), concentrate volume, supernatant $\mathrm{pH}$ and residual leukocytes in clinical practice, although single test criteria deviate, e.g. the required $\mathrm{pH}$ in the United States is lower than 6.2, whereas in Germany it varies between 6.4 and 7.8. Routinely applied tests in PCs reflect only a minor part of the platelet changes during storage, also mentioned as the PSL. In addition, all these tests (see Table I-2) only determine the viability and function of platelets in vitro. Many investigators favor the measurement of the percentage recovery and in vivo survival of radiolabeled (generally with

${ }^{51}$ chromium or with ${ }^{111}$ indium), autologous platelets as a gold standard (34). This fact should be considered if altered or new storage conditions and/ or platelet substituents are evaluated (5). In healthy individuals, the mean in vivo recovery of fresh platelets from platelet rich plasma (PRP) amounts to $60-70 \%$, whereas, after 5 days of storage, the mean recovery amounts to $45-50 \%$. Higher recovery rates were observed for apheresis concentrates on the $5^{\text {th }}$ day.

However, an acceptable "standard for approval" by FDA does not exist for platelet recovery and a standardization of practical implementation has not yet been established as compared with the standardized techniques for vesicle measurement (see chapter 1.4.6; page 29). 
Table I-2: In vitro tests of platelet concentrate quality monitoring

Platelet structure

Functional tests

Metabolic status

Platelet activation

Possibility of transfusion reactions (TR)
Cellular content (platelet count)

Visual inspection of swirling phenomena

Platelet morphology by microscopy

Platelet size distribution by automated counters (PMV, PDW)

Measurement of reticulated platelets

Immature platelet fraction (IPF)

Platelet aggregation, spontaneous and agonist-directed

Hypotonic shock response (HSP)

Extent of shape change

Thrombin-stimulated ATP release

Supernatant $\mathrm{pH}, \mathrm{pO}_{2}, \mathrm{pO}_{2}, \mathrm{HCO}_{3}$

Glucose consumption

Lactate production

p-selectin (CD62P) surface expression

Soluble $p$-selectin release into supernatant

Platelet factor 4 and $\beta$-thromboglobulin

Annexin $\mathrm{V}$ binding to estimate the reorganization of

phosphatidylserine (PS) exposure

Lactate dehydrogenase (LDH) release into supernatant

Platelet microparticle formation

Bacteriological growth

Cytokines

Activated complement components, i.e. C3a

Residual leukocytes

Residual red blood cells (RBC) 
Two in vitro tests should be explained as examples for all tests listed in Table I-2. The simplest evaluations include visual examination of PCs before they are applied to patients. Upon exposing of the storage bag to any light source, a "swirling" phenomenon occurs due to the fact that resting platelets are discoid and refract light within the moved storage bag. If $\mathrm{pH}$ of stored PCs drops below the value of 6.2 or platelets underlie activation, the former discoid/ spherical shape of platelets undergoes morphological changes with formation of pseudopodia. Shape change causes the disappearance of the "swirling" phenomenon caused by the fact that shapechanged platelets are not able to refract light (5). The weaker "swirling" phenomenon correlates to a lower platelet increment in vivo which is measurable after transfusion $(5,35)$. This happens because $\mathrm{pH}$ lower than 6.3 translates to diminished in vivo survival after transfusion (5). The subsequent discoid/ spherical conversion and microvesiculation result in decrease of mean platelet volume (MPV) (17). MPV is a common parameter, which is measured by automated hematology blood counters and could be easily implemented in the running procedures of clinical practice to maintain the visualized "swirling" phenomenon, which considerably depends on individuals and status of training.

The analysis of platelet activation during manufacturing by flow cytometry and evaluation of the p-selectin (CD62P) exposure at the platelet surface (36) is another useful measurement of PCQC and it is applied as the most common measurement of platelet activation in PCs (5). In contrast to the physiological behavior of activated platelets, ex vivo p-selectin expression on the platelet surface upon activation by cold or pH below 6.2 is not reversible (37). In whole blood and platelet-rich-plasma (PRP), p-selectin expression amounted to $2-10 \%$ versus $20-30 \%$ in PCs (37). The percentage of CD62P positive platelets significantly increased within five days of storage, whereas the mean fluorescent intensity (MFI) values of labeled lysosome-associated membrane proteins 1 and 2 (LAMP-1, LAMP-2) decreased. Simultaneously, the reduction of CD62P positive platelets, LAMP-1 and LAMP-2 on the sixths day of storage was observed (36). The relative microparticle count and p-selectin expression on platelets surface were also determined and an increased MV number over the storage time in correlation to free and membrane-bound p-selectin was demonstrated. In general, the activation of platelets entails the occurrence of adverse effects during storage, but it is hardly evident that the rate of activation is associated with post-transfusion recovery (5). Several studies reported that p-selectin expression does not affect platelet clearance $(38,39)$ and may not reflect platelet survival and function in vivo (5).

To highlight the importance of the applied tests for plateletpheresis concentrate quality monitoring, all subsequent parameters, which are beyond standard quality control (see Table I-3) for testing platelet viability at the university hospital of Regensburg, are described later (chapter 4.6; page 51). More parameters are necessary to guarantee platelet integrity in concentrates and to promote the improvement of cell component based quality control at the cellular, biochemical and molecular level. 
Table 1-3: Parameters and criteria of quality control in manufacturing of plateletpheresis concentrates in Germany

\begin{tabular}{|c|c|c|}
\hline Test parameters & Test criteria & Time of testing \\
\hline Volume & according to specifications & after preparation \\
\hline Platelet count per unit & $>2 \times 10^{11} /$ unit & $\begin{array}{l}\text { after preparation and } \\
\text { at the end of expiry }\end{array}$ \\
\hline Platelet count per mL & according to approval & after preparation \\
\hline Residual erythrocytes & $<1 \times 10^{6} /$ unit & after preparation \\
\hline Residual leukocytes & $<3 \times 10^{9} /$ unit & after preparation \\
\hline $\mathrm{pH}$ value & $6.4-7.8$ & at the end of expiry \\
\hline Visual valuation & $\begin{array}{l}\text { undamaged PC container and } \\
\text { swirling }\end{array}$ & $\begin{array}{l}\text { at the end of expiry and } \\
\text { before application }\end{array}$ \\
\hline Sterility & sterile & at the end of expiry \\
\hline
\end{tabular}

\subsection{Extracellular vesicles in general}

\subsubsection{Definition}

Under physiological or pathological conditions, all human body fluids, such as plasma (40), serum (41), breast milk (42), amniotic fluid (43) or urine (44) in vivo, as well as media from cultured cells in vitro (45) contain cell-derived vesicles. Cell membrane vesicles, which undergo secretion, constitute spherical or tubular structures surrounded by a phospholipid bilayer. Vesicular membranes expose receptors that are constructed as transmembrane proteins with cytosolic components, i.e. soluble hydrophilic or hydrophobic molecules. Because the membrane orientation of cell-derived vesicles is the same as that of the donor cells, vesicles can be considered as non-nucleated miniature versions of a cell (46). Both, eukaryotic and prokaryotic cells have the ability to release such vesicles. The characteristics of vesicles are determined by size, density, visibility under the microscope, sedimentation, lipid composition, main protein markers and intracellular membranes or organelles of subcellular origin $(46,47)$. 
Cell-derived vesicles possess different physiochemical features. Therefore, there is currently no consensus about a uniform nomenclature for all cell-derived vesicles. While scientists still debate the nomenclature, the term "extracellular vesicles (EVs)" has been proposed as a general term covering the complete diversity of vesicles. In relation to the current nomenclature, the International Society for Extracellular Vesicles (ISEV) meanders through multiple definitions of vesicles and offers suggestions for scientists and authors. In addition, a general term of EVs used for subgroups should base on logical arguments referring to physiochemical and functional characteristics and should clearly define the methods for isolation and measurement (48).

There are two subgroups of EVs, i.e. exosomes and microvesicles. Apoptotic vesicles form a third, separate class $(46,47)$. There are more types of vesicles described, such as endosomes, ectosomes, membrane particles or exosome-like vesicles (46). This division is based on different characteristics explained above. The association of the latter four types with plateletderived vesicles is currently unknown and therefore not discussed in this study. The size of the largest subgroup of vesicles, i.e. apoptotic bodies, released during apoptotic cell death, ranges from $500 \mathrm{~nm}$ to $5 \mu \mathrm{m}$ (46); (see Figure I-3 and Table I-4).

Microvesicles of a size ranging between $100 \mathrm{~nm}$ to $1000 \mathrm{~nm}$ (46) are released from the plasma membrane during cell stress. The smallest vesicles, also known as exosomes, vary in their size from 50 to $100 \mathrm{~nm}$ (46). They are formed from intracellular multivesicular bodies (MVB; sometimes also used as multivesicular endosomes, MVE) in a process described as the "classic pathway" (47) or produced in a direct pathway $(46,49)$.

The characteristics of these three subtypes (apoptotic vesicles, microvesicles and exosomes) are summarized in Table I-4. The efficient purification of vesicle subgroups is limited (50) and depends on isolation strategies. As a means to interpret the analytical results, it is necessary to assume a small contamination of vesicles neighbored to the refined vesicle subgroup. Rather, the vesicle subgroups represent a "continuum of vesicle types with overlapping properties [which are almost entirely] present in body fluids" (47). It should be considered that "ex vivo purified vesicles" can be "artificially generated during manual dissociation of tissues" (46) and that exosomes could represent intracellular multivesicular bodies, released from ruptured vesicles and non-secreted exosomes. 


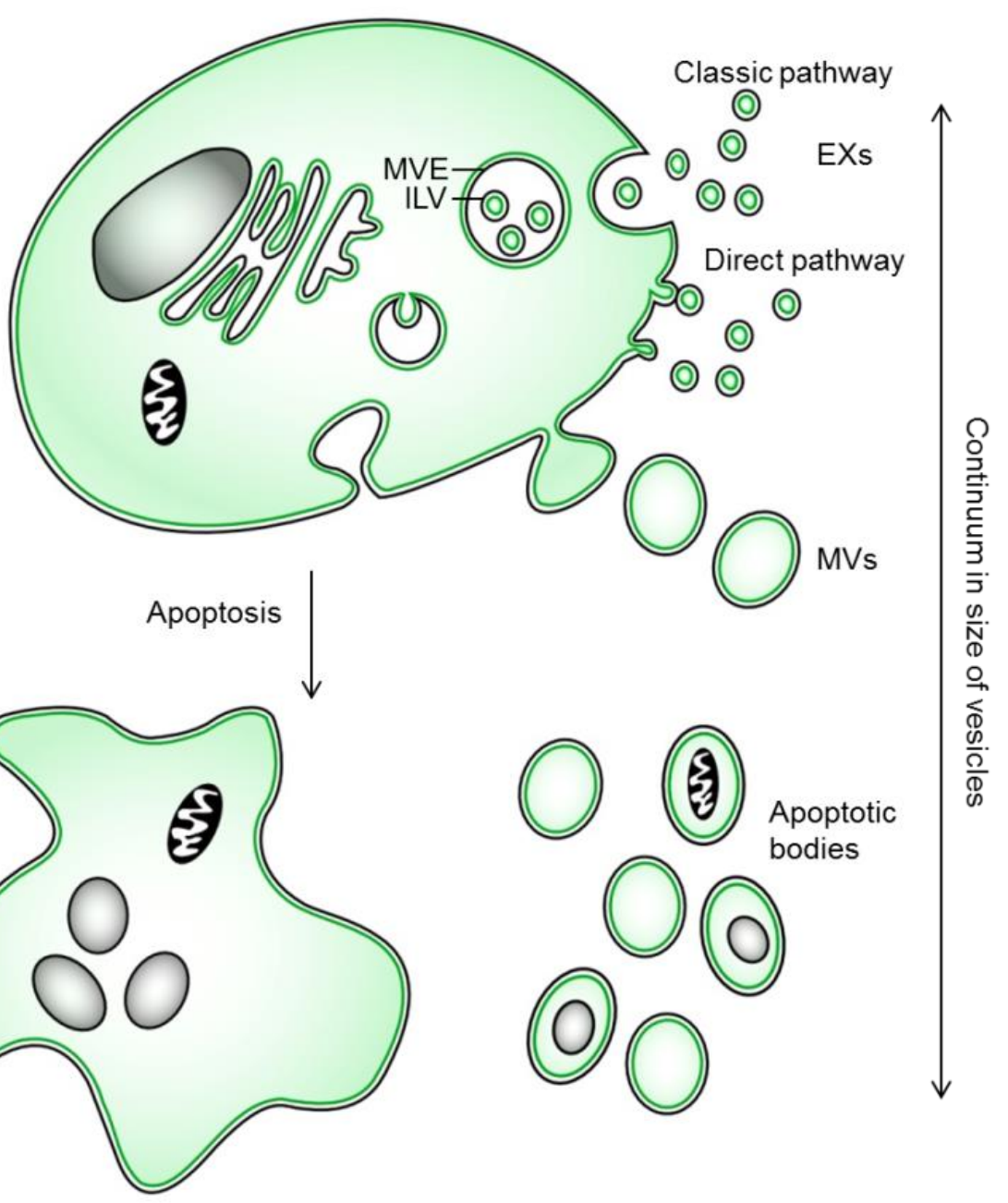

Figure I-3: Scheme of eukaryotic cell forming microvesicles, exosomes and apoptotic bodies

Cells release small exosomes (EXs) via a classic pathway involving formation of intraluminal vesicles (ILV) within multivesicular endosomes (MVE) and via a direct pathway from the plasma membrane. Larger, secreted microvesicles (MVs) can be formed at the plasma membrane by direct budding. Apoptotic bodies including cellular compartments appear during apoptosis. 
Table I-4: Overview of three subgroups of extracellular vesicles

\begin{tabular}{|c|c|c|c|}
\hline & Exosomes & Microvesicles & Apoptotic vesicles \\
\hline $\begin{array}{l}\text { Size of diameter } \\
\text { [nm] }\end{array}$ & $50-100$ & $\begin{array}{l}100-1000 \text { or } \\
20-1000\end{array}$ & $\begin{array}{l}50-500 \text { or } \\
1000-5000\end{array}$ \\
\hline $\begin{array}{l}\text { Density in } \\
\text { sucrose }[\mathrm{g} / \mathrm{ml}]\end{array}$ & $1.13-1.19$ & unknown & $1.16-1.28$ \\
\hline Sedimentation & $100,000 \mathrm{~g}$ & $10,000 \mathrm{~g}$ & $\begin{array}{l}1,200 \mathrm{~g}, 10,000 \mathrm{~g} \text { or } \\
100,000 \mathrm{~g}\end{array}$ \\
\hline $\begin{array}{l}\text { Morphology by } \\
E^{*}\end{array}$ & $\begin{array}{l}\text { cup-shaped, } \\
\text { homogenous }\end{array}$ & $\begin{array}{l}\text { irregular shape, } \\
\text { electron-dense }\end{array}$ & heterogeneous \\
\hline Cellular origin & most cell types & most cell types & all cell types \\
\hline Cell origin & multivesicular bodies & plasma membrane & $\begin{array}{l}\text { plasma membrane } \\
\text { and endoplasmic } \\
\text { reticulum during cell } \\
\text { death }\end{array}$ \\
\hline Composition & $\begin{array}{l}\text { cholesterol, } \\
\text { sphingomyelin, ceramide, } \\
\text { lipid rafts, } \\
\text { glycerophospholipids, } \\
\text { phophatidylserine- } \\
\text { exposure, Rab proteins, } \\
\text { annexin, flotillin, intergins } \\
\text { and tetraspanins (CD63, } \\
\text { CD9, CD81, CD82), Alix, } \\
\text { TSG101, HSP (hsc70, } \\
\text { hsc90), mRNA, microRNA }\end{array}$ & $\begin{array}{l}\text { phosphatidylserine- } \\
\text { exposure, intergrins, } \\
\text { selectins and CD40 } \\
\text { ligand; insufficiently } \\
\text { known }\end{array}$ & $\begin{array}{l}\text { histone, DNA, } \\
\text { organelles }\end{array}$ \\
\hline References & $(27,46,47,51-54)$ & $(46,47,54,55)$ & $(46,47,56-59)$ \\
\hline
\end{tabular}




\subsubsection{Function of extracellular vesicles}

The EV subgroups are released by cells during different stages of cell life. Thereby, EVs reflect dissimilar formation processes and are related to different mechanisms of shedding of intracellular and/ or plasma membrane components into the intercellular environment. Released EVs are recognized by acceptor cells. It is obvious, that both, donor and acceptor cells benefit from the transposition of these cell fragments, where donor cells release vesicles, whereas target cells, which are brought into contact with the released vesicles, are acceptors. There is an increasing interest in functions of EVs and the most relevant properties of EVs shall be elucidated.

In general, cell-derived vesicles may act as "multi-purpose" carriers (45), (see Figure I-4) to express the main functions of extracellular vesicles discussed in this work.

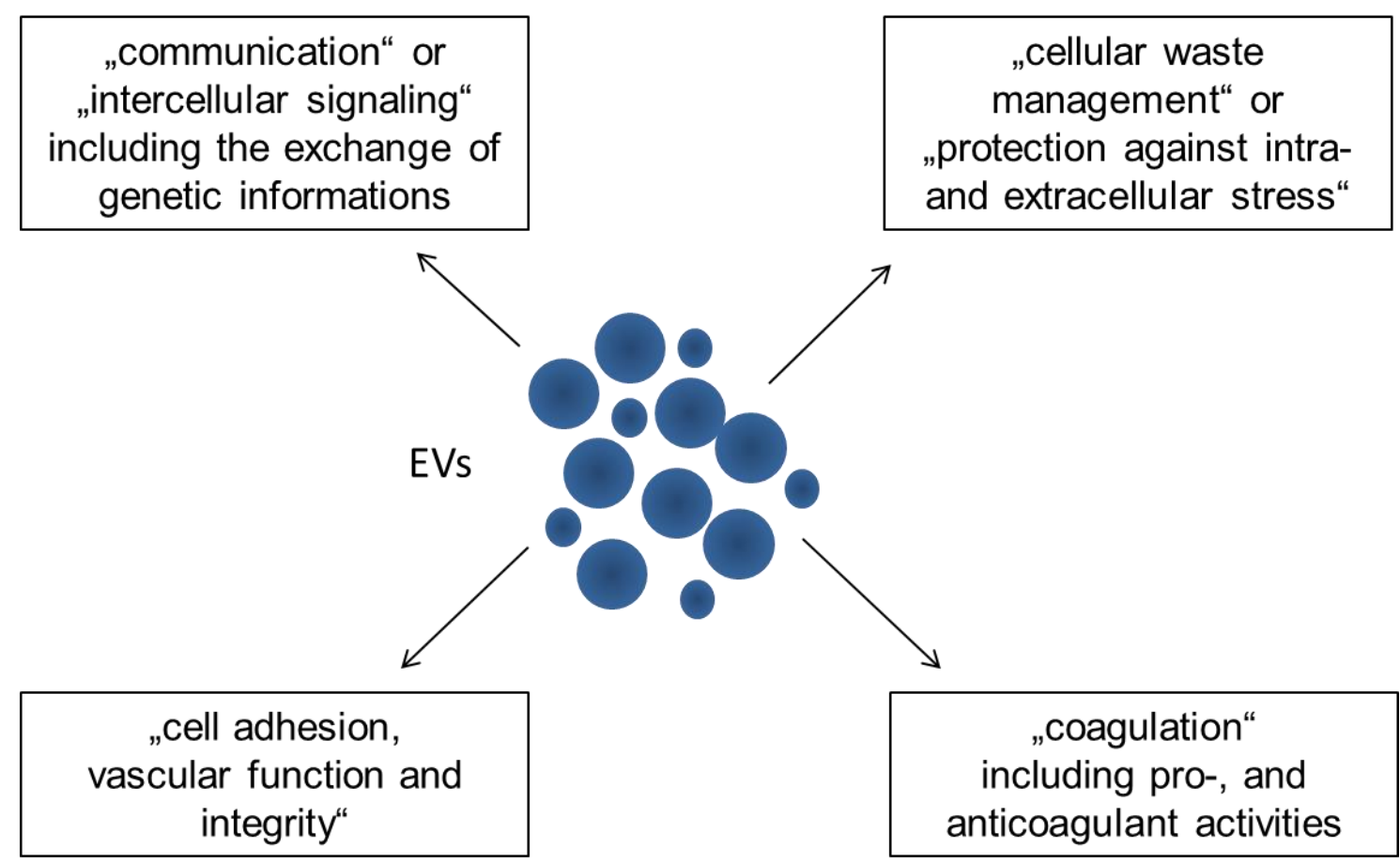

Figure I-4: Summary of the main functions of extracellular vesicles (EVs)

As indicated, the process of intercellular signaling via vesicles as potential communicators and, thus, understanding the function of cell-derived vesicles is an important current topic. The transport of receptors or cytokines was characterized and found to be similar to the transfer of the TF receptor and p-selectin glycoprotein ligand-1 (PSGL-1) between leukocyte-derived vesicles and activated platelets. As a result of this transfer, proteolytic activity of the TF-VIla complex increases after fusion of leukocyte-derived microvesicles and platelets (60). 
The transfer of ligands, e.g. CD95L (FASL), located on tumor cell-derived vesicles induced apoptosis of $T$ cells (61) which is a proof for immunosuppressive effects of the intercellular communication.

The mechanism of promotion of humoral immune response via CD40L (CD154) of plateletderived vesicles followed by $B$ cell activation and production of immunoglobulin $G$ can be considered as sufficiently understood (62). The communication aspect involves an "orchestrating function" of immune response including antigen presentation and the transfer of cellular components.

A further possibility of the communication between released vesicles and cells is defined through the exchange of genetic information. This was shown in many tumor cell lines, e.g. transfer of functional mRNA and miRNA in vesicles from glioblastoma cells (63), or mRNA in colorectal cancer cell line-derived vesicles (64) and in physiological cells, e.g. miRNA from T cells (65) or miRNA from dendritic cells (DCs) (66). The exchange of functional miRNA among DCs results in the repression of target mRNA in acceptor cells. This may be an important key mechanism in saving the function of non-coding regulatory miRNA of cellular origin outside the cell, in which it was produced, because, as in the case of vesicles, miRNA is protected from degradation carried out by RNases (67). The ability of vesicles to facilitate the transfer of functional genetic information was pictured in exosomes of mouse and human mast cell lines and primary bone marrow-derived mouse mast cells. Exosomes contain functional mRNA of approximately 1300 genes undetected in the cytoplasm of the donor cells and miRNA called "exosomal shuttle RNA" (esRNA) (27). Induced expression of target proteins was also observed. Lässer's work presented detectable levels of RNA in vesicles of several body fluids, e.g. human saliva, plasma or milk (68). These findings indicate a flow of information through RNA transfer.

"Cellular waste management" or "protection against intra- and extracellular stress"(45) using the extracorporeal release of harmful substances in vesicles may be a survival strategy of cells. External stress, e.g. complement C5b-9 complex incubated with platelets, causes the release of vesicles enriched with this complex to protect the platelets from complement-induced lysis (69). Reticulocyte-derived transferrin receptor (TFR) or tumor cell-derived shedded vesicles, which contain chemotherapeutics, are active in the process of survival or drug resistance mechanisms (70) and were described as extracellular stress protectors.

Active caspase 3 is the main executioner of apoptosis. Caspase 3 is abundant in vesicles of different cell types and it is assumed that it acts as a protection against internal stress and nemesis of cells $(71,72)$.

Vesicles also play a role in cell adhesion, vascular integrity and repair which results from their expression of adhesion molecules and from the increasingly significant relation of the surface to cell compartment. "All membrane vesicles of any cellular origin express adhesion molecules on their surface, which could favor their capture by recipient cells" (46). 
Platelet-derived vesicles were observed how they bind to fibrinogen-, fibronectin-, and collagencoated surfaces and minimally to vitronectin and von Willebrand factor (73). In a rabbit model, an increased vesicle binding to injured endothelium in vivo, but not to uninjured surfaces, was observed and elevated binding of platelets to immobilized vesicles was shown. This behavior was interpreted as a mechanism by which vesicles promote thrombus formation.

Another example of exosome-mediated adhesion was described for integrins on B cell-derived exosomes, which interact with extracellular matrix components to mediate adhesion to collagen-I, fibronectin and surface adhesion molecules of tumor necrosis factor (TNF)- $\alpha$ activated fibroblasts (74). Similar to the mechanism of protection against intracellular stress through caspase 3 activity, an increased release of endothelial-derived vesicles antagonize apoptotic blebs. Tumor susceptibility gene 101 and translation controlled tumor protein (TCTP), which are abundant in vesicles, trigger an extracellular signal-regulated kinase 1/2 (ERK1/2)dependent antiapoptotic phenotype in vascular smooth muscle cells (VSMCs) (75). In contrast to the antiapoptotic effect, the vesicles from endotoxin-stimulated monocytes trigger caspase 1 dependent apoptosis of vascular smooth muscle cells.

These observations suggest functions beyond the communication between cells and vesicles, and underline the role of vesicles in damage control of vasculature and a potential role of vesicles in coagulation. Section 1.4.1 summarizes historical information on potential coagulatory features under clinical aspects of vesicles.

Two topics referring to coagulation should be discussed. The first topic should concentrate on vesicles as specific or unspecific procoagulants and the second topic should refer to vesicles with anticoagulant activity. In the context of microvesicle-related coagulability, the results of analyses of the material of patients with Castaman syndrome are of interest $(76,77)$. In four unrelated patients with life-long bleeding tendency, a reduced formation of platelet microvesicles was found. These patients were without von Willebrand factor defect and with no evidence of any other platelet function abnormalities accompanied by normal prothrombin consumption. Castaman syndrome, which is at variance with Scott syndrome (see in 1.3.3), was described as disorder in vesicle formation and the EV generation was closely connected with coagulability under physiological conditions. In addition, the procoagulant activity of EVs results from the molecular composition of the vesicle membrane including presentation of anionic phospholipids, e.g. phosphatidylserine, in the outer monolayer (78). This corresponds to positively charged $y$-carboxyglutamic acid (GLA) domains in proteins of the clotting system, such as factors VII, IX, X and II (prothrombin) (79). There is no consensus concerning PS exposure on exosomes and microvesicles. However, PS-positive EVs $(51,80)$ and PS-negative EVs $(54,81,82)$ were reported in context of several diseases.

Tissue factor, as a cofactor of the initiation of the extrinsic coagulation cascade by factor VIla, is also present as a procoagulant on the surface of EVs. The TF/FVIla complex, however, is counter-regulated by tissue factor pathway inhibitor (TFPI), suggesting that not all TF/FVIla 
complexes on EVs remain active (or become activated) $(79,83,84)$. Similarly to PS exposing EVs, the procoagulant activity of TF bearing EVs remains also controversial. The pro- or anticoagulant activity of exosomes and, most likely, of microvesicles depend on physiologic or pathologic conditions (85). It was reported that TF exposing exosomes and microparticles from human saliva, promotes a shortened clotting time in human pericardial wound blood (85). Microvesicles triggered coagulation in a TF-depending pathway. The same working group showed that microvesicles from platelets, erythrocytes and granulocytes in blood samples also support coagulation via a TF-independent way. Antibodies against TF or FVII were ineffective to stop coagulation, suggesting PS-exposure as an initial step of coagulation.

Further, an anticoagulant function was concluded. An inverse correlation of annexin V-positive microparticles and thrombin generation to levels of thrombin-antithrombin-complexes was found. Thrombin in a lower concentration activated protein $C$ and led to an anticoagulant effect of microvesicles (86). PS-positive vesicles present in blood of healthy individuals predominantly originate from platelets, megakaryocytes, and the surface of platelet-derived vesicles showed 50 - to 100 -fold higher procoagulant activity than that of a single platelet (87). Monocytes, however, are likely to be the major donor cells of TF-positive vesicles (79). Moreover, in several diseases, the procoagulant activity of vesicles, e.g. tumor-derived vesicles exposing TF, was found associated with an increased risk of venous thromboembolism (VTE) $(79,88)$. An increased incidence of thrombotic events was observed in patients with paroxysmal nocturnal hemoglobinuria $(\mathrm{PNH})$ with ascending concentrations of microparticles (89). In contrast to these procoagulant activities, the results of TF-exposing microparticles in patients with multiple organ dysfunction syndrome (MODS) and sepsis indicate an inverse effect. Lower numbers of vesicles in these patients could explain this effect (82). Altogether, PS- and TF-bearing EVs play an undisputed role in hemostasis and thrombosis, but the contribution of exosomes or microvesicles is still unclear.

\subsubsection{Microvesicle formation}

Lipid translocases, ions and their plasma membrane channels, as well as such processes as cytoskeletal reorganization, signal transduction, lipid translocase-independent and apoptotic mechanisms are all involved in vesicle formation (78). The role of translocases in vesicle formation and shedding is coupled to the loss of asymmetric distribution of lipids in the outer and inner plasma membrane (90). Exposure of PS towards the outer cell membrane by an ATPdependent protein "floppase" (91) is an initial event in shedding of membrane vesicles (92). A normal bilayer membrane consists of positively charged polar phospholipids, such as phosphatidylcholine and sphingomyelin in the outer leaflet, and negatively charged anionic phospholipids in the inner leaflet, e.g. aminophospholipids, phophatidylserine (PS) or phosphatidylethanolamine (PE) (93). Aminophospholipid translocases with "flippase" activity direct PS and PE back to the inner leaflet of the cell membrane, whereas lipid scramblase is responsible for a bi-directional transfer of phospholipids and thus maintaining membrane 
integrity (91). Scramblase is also the base of a rapid PS exposure towards the cell surface as a part of the physiologic hemostasis in coagulation response $(94,95)$.

As far as the lipid transport mechanisms are concerned, stimulation-dependent increase in cytosolic $\mathrm{Ca}^{2+}$ provokes a disruption of membrane lipid distribution by activation of scramblases and floppases, whereas flippase activity is inhibited (96). Scott Syndrome is a bleeding disorder characterized by a deficiency in floppase activity, impaired shedding of vesicles and decreased PS expression on vascular cells $(97,98)$. Sorting of membrane compartments into the vesicle bleb may be regulated by lipid rafts of the cell membrane (99). The activation of the $\mathrm{Ca}^{2+}$ dependent protease calpain after intracellular influx of calcium ions induces a release of microvesicles from platelets (78) and also promotes proteolysis of the cytoskeleton, which subsequently induces the formation of vesicles (92). The entry of calcium seems to be a common mechanism in platelet activation, while in the resting state of platelets a steady calcium $\left[\mathrm{Ca}^{2+}\right]_{i}$ is maintained by the endoplasmic reticulum (ER) as a calcium store.

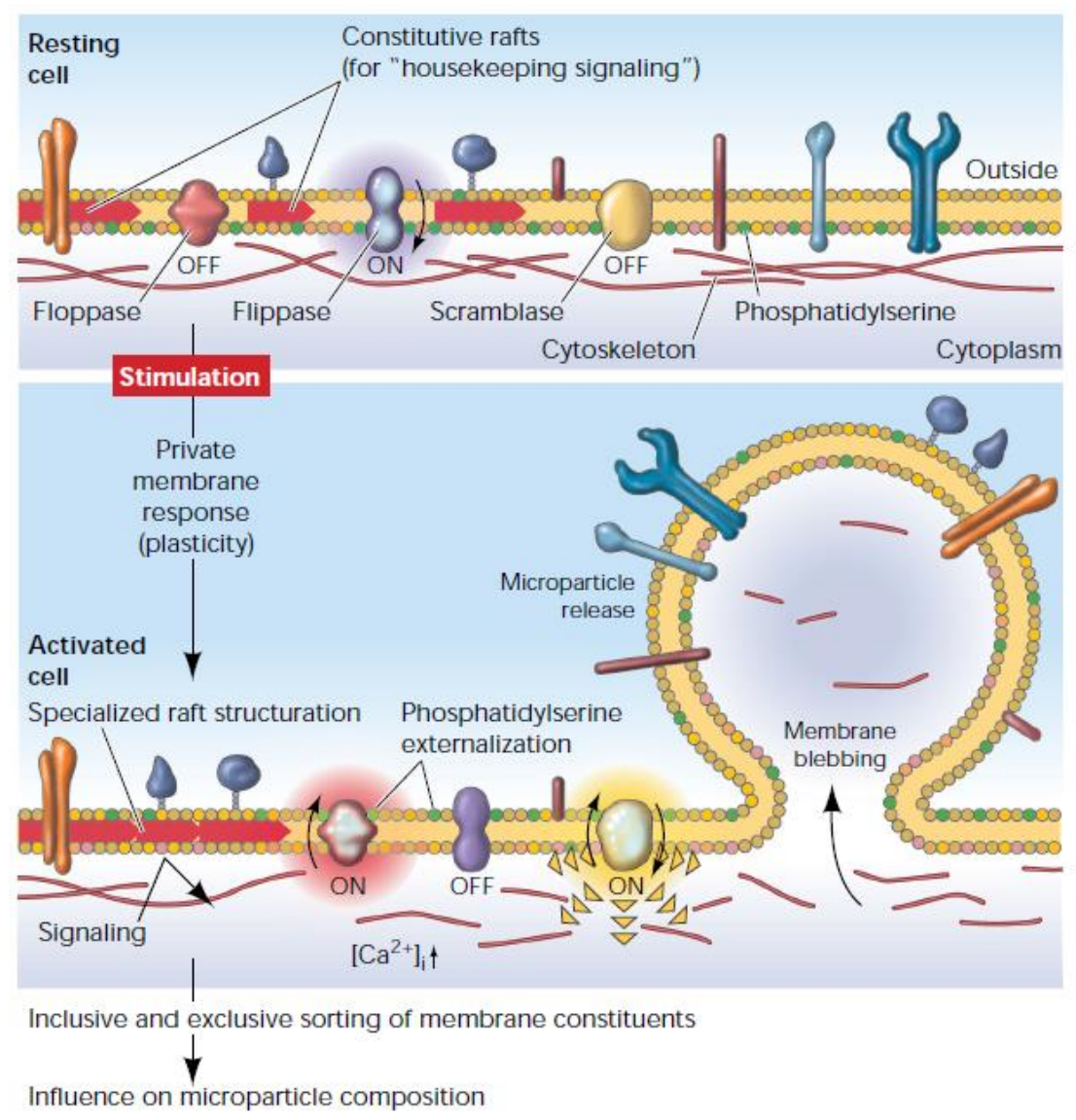

Figure 1-5: The formation of microvesicles from the cell membrane

Upon stimulation of resting cells, the cytosolic $\mathrm{Ca}^{2+}$ level increases, the activation of floppases and scramblases and inhibition of flippases result in the externalization of phophatidylserine on the outer leaflet of the cell membrane. Lipid and protein rafts may control the sorting of membrane compartments, which are excluded from the vesicle blebs (figure from (100)). 
There are two mechanisms which regulate cytosolic calcium $\left[\mathrm{Ca}^{2+}\right]_{i}$ in platelets. The first mechanism is based on the calcium release from the cytosol and on the influx of $\mathrm{Ca}^{2+}$ through the cell membrane mediated by store-operated calcium entry (SOCE). The second mechanism involves the ligand-gated ion channel $P 2 X_{1}$ (purinergic receptor $P 2 X_{1}$ ) (101). In monocytes, $\mathrm{P}_{2} \mathrm{X}_{7}$ receptor (purinergic receptor $\mathrm{P} 2 \mathrm{X}$, ligand-gated ion channel 7 ) can be activated by extracellular ATP (102).

The calcium entry via SOCE mechanism may act as a modulator of PS exposure and of a refilling of the ER calcium stores $(103,104)$. To maintain thousand fold lower concentration of $\left[\mathrm{Ca}^{2+}\right]_{i}$ than in the extracellular space or in the intracellular $\mathrm{Ca}^{2+}$ pools, $\mathrm{Ca}^{2+}$-ATPases translocate $\mathrm{Ca}^{2+}$ across the cell membrane using the energy of ATP hydrolysis. In platelets, different isoforms of sarco/ endoplasmic reticulum $\mathrm{Ca}^{2+}$-ATPases (SERCAs) have been found. They become activated through the intracellular $\mathrm{Ca}^{2+}$ influx and pump $\mathrm{Ca}^{2+}$ back into the intracellular $\mathrm{Ca}^{2+}$ pools (105). Other key proteins acting as ion channels are involved in the store-operated calcium entry (SOCE). As store-operated calcium (SOC) channels, the stromal interaction molecule 1 (STIM1) and further in the system of tubular denses the Orai1 (also known as $\mathrm{CRACM} 1$ = calcium release-activated calcium channel protein/ modulator 1) were detected (106). Both, the STIM1/Orai1 pathway and the thrombin receptor pathway (54) operate in platelets on $\mathrm{Ca}^{2+}$ influx and PS externalization, together with SOCE-independent and celltype specific $\mathrm{Ca}^{2+}$ signaling, as well as other ions such as $\mathrm{K}^{+}, \mathrm{Cl}^{-}, \mathrm{H}^{+}$, and $\mathrm{Na}^{+}(78)$.

Destabilization of the actin cytoskeleton in platelets is stimulated by the allbß3 signaling pathway and by calpain activation accompanied by high concentrations of $\mathrm{Ca}^{2+}$ (107). Caspases, as dominant regulators of the apoptosis cascade, are involved in the reorganization of the cytoskeleton (78). Proteases, caspases and calpain dominate the proteolysis of filamin-1, talin and myosin (108). Recent studies suggest that Rho-associated coiled-coil-containing protein kinase (ROCK) II, activated by caspase-2 in endothelial cells, is linked to the cytoskeletal reorganization, release of microvesicles $(109,110)$ and may be related to inflammation.

Signal transduction pathways regulate formation and secretion of microvesicles from activated cells. Examples are provided in a comprehensive review of Morel who demonstrated that platelet-derived microvesicle formation is associated with phosphorylation of platelet proteins by calmodulin, myosin light chain kinase, and other calmodulin-regulated effectors. This microvesicle formation process was found to be similar to the pathway of activation-induced protein tyrosine dephosphorylation (78). Results from a study on endothelial cells showed reduced microvesicle formation induced by TNF- $\alpha$ through inhibition of p38 mitogen-activated protein kinase (111). In fact, the release of microvesicles is regulated by stimuli which activate cell specific receptors, e.g. Toll-like receptor 4 (TLR4), the receptor for gram-negative bacterial lipopolysaccharide (LPS) on DCs (112) or on platelets (113), which may influence vesicle formation. 
Platelets bound to LPS via TLR4 show increased phosphorylation of p38 mitogen-activated protein kinase and decreased production of prothrombotic PS and CD62P-positive microparticles in response to thrombin receptor agonist peptide (TRAP). Therefore, a direct activation by TLR4 reduces the TRAP-stimulated platelet vesicle formation in vitro. An enhanced thrombotic risk of bacterial infections is likely to reflect the TLR4 activation of other cells which indirectly stimulate platelets through chemokines/ cytokines (113). Pathways involved in microvesicle formation are determined by a cell lineage and a type of cellular stress (78).

In addition, translocase-independent mechanisms of vesicle and transient pore formation in the cell membrane and blebbing mechanisms may be responsible for $\mathrm{Ca}^{2+}$, PS -independent formation of MV $(114,115)$.

During apoptosis, PS occurrence is one of the first changes, prior to DNA fragmentation, leading to the loss of asymmetry of cell membranes $(116,117)$.

In apoptotic cell death, several regulators, including caspases (118), which affect apoptosis in nucleated cells, and various events such as loss of mitochondrial membrane potential, PS exposure and contraction of cells, are assumed to trigger apoptosis (119). In platelets, members of the Bcl-2 apoptosis inhibitory protein family are also present and Bcl-xl, a member of the Bcl2 protein family, plays a role in procoagulant activity of platelets and their viability (119). However, the extent to which the effects of apoptosis and activation affect formation of vesicles is poorly understood. The process of membrane blebbing is associated with phosphorylation of myosin light chain and ROCK I activation due to the cleavage by caspase-3 during apoptosis (120).

\subsubsection{Formation of exosomes}

Exosomes mainly originate from the lumen of multivesicular endosomes (MVE) as forms of intraluminal vesicles (ILV) which are released from MVEs after fusion of their membrane with the cell membrane. Different types of MVEs exist. Some MVEs are subjected to degradation and subsequently lysed by fusion with lysosomes; other MVEs fuse with the outer cell membrane to release their ILVs as exosomes. This MVE secretion pathway was described as the "classical pathway of exosome formation" (47) but consecutive sorting mechanisms have not been elucidated. The endosomal sorting complexes required for transport (ESCRT) execute sorting of transmembrane receptors for vesicles which are predetermined for degradation (121, 122). In dendritic cells, Hrs, an ESCRT-0 protein, was involved in the formation of DC-derived endosomes (123). On the other hand, data from a mouse oligodendrogial cell line provide evidence for an ESCRT-independent pathway of MVE and exosome formation, which may depend on ceramide microdomains (124). Further differences in the degradation and secretion pathways are evident. Cholesterol labeling was found only in the plasma membrane of lymphoblastoid cells and in MVEs, and in exosomes after secretion (125), but it was absent in 
Golgi cisternae and the surrounding membrane of MVBs. The late endosomal marker bis(monoacyl)-glycerophosphate (BMP) is also involved in the formation of vesicles predestined for degradation in intraluminal MVEs, but is not involved in the sorting of exosomes (126). BMPnegative MVBs are exosomal targets for epidermal growth factor (EGF) and the EGF receptor (EGFR) (127), whereas MVEs containing BMP are not trafficking EGF and EGFR and are assigned for lysosomal degradation.

More cytosolic and membrane proteins and lipids are thought to play a role in sorting of exosomes. Endosomes, for example, are formed in an ESCRT-independent manner (128) through other proteins, e.g. tetraspanins CD63 and CD9, or without ubiquination of major histocompatibility complex (MHC) class II molecules on DCs (129). In conclusion, various mechanisms are involved in vesicle sorting. Based on the stimulus-dependent secretion or recycling to MVEs, exosomes employ different sorting ways in different cell types, cell compartments and at various stages of cell maturation.

Trafficking and fusion with the plasma membrane require small guanosine triphosphate (GTP)ases of the Ras-related in brain (Rab) protein family. Rab11 and calcium are involved in MVE fusion from K562 erythroleukaemia cells (130). Rab27a and Rab27b were found in the plasma membrane docking mechanism in HeLa cells (131) and GTP-dependent Rab35 was present on the surface of oligodendrogial cells with an impaired secretion of exosomes (132). The soluble $\mathrm{N}$-ethylmaleimide-sensitive-factor attachment receptor (SNARE) proteins may be involved in the fusion of MVBs for exosome release, this pathway, however, is specific for lysosomal secretion (133).

Beside the classical pathway, exosome release is mediated by a direct fusion of small vesicles with the cell membrane. In this case, exosomes do not originate from MVEs (47). In relation to this phenomenon, the difference between exosomes originating from MVEs or exosomes released directly from the cell membrane is unidentifiable. It was observed, that the HIV accessory protein negative factor (Nef) (134) and diverse Gag (group-specific antigen) proteins $(135,136)$ possess sorting information on exosome release in Jurkat T cells and T cells. "The extent to which such exosomes are also released from other cells or in vivo (e.g., in biological fluids) is unknown" (47).

\section{$1.4 \quad$ Platelet-derived extracellular vesicles}

\subsubsection{Background and clinical potential}

At least since the 1940s, platelets have been considered to play an important role in hemostasis. According to newer reports, however, a thromboplastic protein fraction, sedimentable by high-speed centrifugation, was also discussed as a potential stimulus of coagulation (137). 
In 1967, PL-EVs were observed for the first time and called "platelet dust" (8). The procoagulant effects of platelet-derived subcellular fragments, distinguishable from effects of platelets, were investigated by means of clotting time. The capability of clotting serum was attributed to PLEVs, which were obtained from platelet-poor plasma separated by ultracentrifugation (UC).

In 1972, Warren et al. observed platelet-derived vesicles which originated from "multivesicular membrane sacs" of dendritic platelet pseudopodia. The way of formation was identified with the help of electron microscopy during adhesion of platelets to the subendothelial layer of coronary arteries in man (138). The investigation confirmed the hypothesis, supported by the results of fixation techniques, that "during the activation of human platelets, the release of membraneassociated multivesicular structures may occur concurrently with other mechanisms of secretory granule content release" (139).

Until now, these extracellular vesicles (48), (in this case, derived from platelets and hence named platelet-derived extracellular vesicles (PL-EVs) (9)), have proven their thrombogenic properties $(9,47,82,86,140-142)$. Moreover, studies that are more recent, demonstrated that platelet-derived vesicles might transport and deliver various molecules among cells $(9,143$, 144). Platelet-derived vesicles also participate in innate $(145,146)$ and adapted $(62,147)$ immunity, in inflammation (148), malignancies (149) and in hematopoietic stem cell engraftment (150). They promote endothelial repair and support angiogenesis (151). The participation in several biological pathways was confirmed mainly in healthy individuals or in patients with cardiovascular and inflammatory diseases or cancer (see Table I-5).

Table 1-5: Platelet-derived vesicles in several diseases

Diseases

Changes in the level of PL-EVs

References

\section{Cardiovascular diseases}

Coronary heart disease

Acute coronary syndrome (ACS)

$\uparrow$

$\uparrow$

Peripheral arterial disease

Severe hypertension

Pulmonary hypertension

Acute pulmonary embolism

Valvular atrial fibrillation

Deep vein thrombosis (DVT)
$(156,157)$ 
continuation of Table 1-5: Platelet-derived vesicles in several diseases

Diseases

\section{Cerebrovascular diseases}

Ischemic stroke

$(163,164)$

Cerebral vasospasm

Lacunar infarcts

Multi-infarct dementia

Carotid atherosclerosis

\section{Hematologic diseases}

Immune thrombocytopenia

purpura (ITP)

Thrombotic thrombocytopenic

purpura (TTP)

hemolytic uremic syndrome

(HUS)

Paroxysmal nocturnal

$(89,169)$

hemoglobinuria $(\mathrm{PNH})$

Heparin-induced

thrombocytopenia

Castaman syndrome

$(76,77)$

Glanzman syndrome

Scott syndrome

Stormorken syndrome

Sickle cell disease

\section{Cancer}

Gastric cancer

Lung cancer

ND

Breast cancer

Prostata cancer

ND

$(181,182)$ 
continuation of Table 1-5: Platelet-derived vesicles in several diseases

Diseases

Infectious diseases

Sepsis

Prion disease

\section{Autoimmune diseases}

Systemic lupus erythematodes

Antiphospholipid syndrome

$\uparrow$

$\uparrow$

Systemic sclerosis

Rheumatoid arthritis

Acute vasculitis

Type 1 diabetes mellitus

Multiple sclerosis

Sjögren syndrome

\section{Other}

Type 2 diabetes mellitus

Metabolic syndrome, central

obesity

Obstructive sleep apnea

Uremia

Cerebral malaria

Decompression sickness

Preeclampsia

Spontaneous abortion

Alzheimer disease

Raynaud's phenomenon
$(186,187)$

$(189,190)$

$(187,191,192)$

(196-199)

$(200,201)$

(206-208)

$(210,211)$

(212)

(Table adapted and supplemented by (213)) 
Further interest in PL-EVs is directed towards their content in blood concentrates, especially in plateletpheresis products. Important investigations are currently performed concerning the level of PL-EVs in PCs in comparison to platelets and the surrounding donor plasma (15) and their potential application to quality control measurements of PCs.

\subsubsection{Distribution of PL-EVs}

In bloodstream of healthy individuals, platelet-derived vesicles are believed to represent the major circulating microparticle fraction amounting to $75-90 \%$ of all circulating microparticles (15, 142). They were found at concentrations of 6 to $300 \times 10 \% \mathrm{~L}$, based on the results of the measurements carried out by the new generation of flow cytometry (FCM) instruments (214). In healthy individuals, the concentration of PL-EVs of circulating platelets under resting conditions constitute approximately $3 \%(172)$, whereas after stimulation with collagen the concentration increases to approximately 32\%. Upon treatment with TRAP-6 (SFLLRN), the PL-EV concentration reaches $53 \%$.

Megakaryocytes may also serve as a source of PL-EV because they express platelet-specific glycoprotein (GP)Illb-IIla $(82,86,215)$. Based on the results of the group of Flaumenhaft, there is no evidence of PL-EV release from resting platelets. Circulating PL-EVs in healthy individuals are likely to originate from activated platelets or from megakaryocytes, however, the latter ones are currently less in the focus of investigations (11). Yet, the obtained results of the differentiation of these two groups of PL-EVs have to be confirmed. Megakaryocyte (MK) derived EVs may serve other functions than PL-EVs derived from activated platelets which are involved in cardiovascular diseases. In patients subjected to autologous or allogeneic hematopoietic stem cell transplantation, MK-EVs should be analyzed in blood samples ex vivo. In patients after total body irradiation, MK-EVs disappeared much faster in comparison to PLEVs and platelets (216). In order to remain consistent with the term of "platelet-derived vesicles", the present work focused only on the release of vesicles from platelets, not from megakaryocytes.

PL-EV levels underlay gender-specific differences (female $>$ male) in platelet-poor plasma (PPP) and platelet-free plasma (PFP) of healthy individuals $(217,218)$ and increase with age (156), during pregnancy (219) and after exercise with increased hemostatic potential $(220,221)$. Lowered levels are described in postmenopausal women with decreased estradiol levels, hormone replacement therapy reverses this effect, though $(222,223)$.

Beside the fact that PL-EVs in the circulation are of diagnostic interest, it is also important to gather the information on the presence, concentration and composition of PL-EVs in platelets. An observation that high concentration of PL-EVs in PCs strongly correlates to PS-exposure and correlates to the use of different apheresis systems was already published in 2000 (224). The enrichment of PL-EVs in PCs is up to 4-fold higher in comparison to the plasma of healthy donors because of EV-formation in either rested platelets or activated platelets. However, the 
majority of EV-subpopulations in PCs stem from resting platelets. Over the storage time, the production of PL-EVs in resting platelets remains low in comparison to the increased level of CD62P-and CD63-positive PL-EVs from activated platelets. Rank also reported a decreased amount of cumulative annexin V-and CD61-positive EVs over the storage time of 5 days (15). In addition to PC-specific, namely platelet-derived vesicles, EVs may also originate from other cell types, i.e. red blood cells or endothelial cells (15). The results of this study confirm the presence of EVs of red blood cell origin.

\subsubsection{Characteristics of PL-EVs}

PL-EVs are released from activated or senescent platelets in the form of nano- or microvesicles, budded from the platelet membrane and in the form of exosomes originating from MVEs and $\alpha-$ granules (54). As mentioned in chapter 1.4.2, the present study does not focus on megakaryocyte-derived vesicles due to their low concentration.

Despite the fact that PS-exposure on activated platelets rather comes from PS of the inner leaflet of platelet membranes than from PS on granules (225), it is not completely clear whether the absence of PS-negative PL-EVs corresponds to rapid clearance or to the low in vitro generation of PL-EVs and megakaryocyte-derived vesicles. Heijnen and colleagues reported that PS-exposure with binding of annexin-V was found mainly on larger PL-MVs with a larger diameter and surface, but not on small PL-EXs (54). Exosomes could partially contribute to the procoagulability of microvesicles.

Common detection markers for PL-EVs are listed in Table 1-6. PL-EVs contain numerous platelet-specific molecules, such as adhesion proteins (e.g. CD42a, CD31, fibrinogen, thrombospondin, vWf) or proteins involved in coagulation (e.g. FVa, FVIII, TF), growths factors (e.g. VEGF, PDGF, TGF- $\beta 1$ ). Additionally, detected complement proteins (e.g. CD55, CD59, C5b-9, C3b, C1q, C1-INH), lipids (e.g. PS, arachidonic acid, LPA, TXA 2 ), immune response molecules (CD40L) and others (e.g. CD63 especially on PL-EXs, lactadherin) classified EVs as platelet-derived compounds (140). 
Table I-6: This overview shows common and variable glycoprotein (GP) receptors or activation markers of platelet-derived and megakaryocyte-derived extracellular vesicles (EVs)

\begin{tabular}{|c|c|c|c|}
\hline & $\begin{array}{l}\text { Platelet- } \\
\text { derived EVs }\end{array}$ & $\begin{array}{l}\text { Megakaryocyte- } \\
\text { derived EVs }\end{array}$ & References \\
\hline $\begin{array}{l}\text { CD41, CD61 (fibrinogen receptor } \\
\text { GPIIb-Illa, integrin allb } \beta 3 \text { ) }\end{array}$ & + & + & $(9,226)$ \\
\hline $\begin{array}{l}\text { CD42b (von Willebrand factor } \\
\text { receptor GPIb) }\end{array}$ & + & + & $(9,226)$ \\
\hline Phosphatidylserine (PS) & + & + & (226) \\
\hline CD63 (GP55) & + & + & $(9,227,228)$ \\
\hline $\begin{array}{l}\text { CD62P (p-selectin) after } \\
\text { stimulation }\end{array}$ & $+^{*}$ & - & (226) \\
\hline CD107a (LAMP-1) after stimulation & $+^{*}$ & - & (226) \\
\hline $\begin{array}{l}\text { Full length filamin A after } \\
\text { stimulation }\end{array}$ & - & + & (226) \\
\hline Cleaved filamin A & $+^{*}$ & - & (226) \\
\hline
\end{tabular}

\subsubsection{Specific features of PL-EV formation}

Current knowledge of PL-EV formation at the molecular level is still incomplete. The present understanding encompasses the formation of PL-EV during the process of platelet activation, platelet senescence, destruction, thrombus degradation and during megakaryocytosis (9). The assumed constitutional production of platelets and smaller cell-derived vesicles from megakaryocytes were discussed earlier (see 1.4.3).

In vitro activated platelets of a normal size up to $2 \mu \mathrm{m}$ release two clearly distinguishable populations of PL-EVs. The first population is constituted of small vesicles $(<100 \mathrm{~nm})$ which originate from MVEs and a-granules and express tetraspanin CD63 assigned to exosomes (EXs). The second population is composed of larger vesicles $(100 \mathrm{~nm}-1 \mu \mathrm{m})$ as microvesicles (MVs; formerly microparticles, MPs) of a typical immunophenotype of platelets and 
megakaryocytes $(47,54,227)$. An overlap of both subtypes is apparent and it depends on the non-uniform isolation protocols and detection techniques of PL-EVs in existing studies and on the fluent transition of membrane reticulation.

Vesiculation follows activation of platelets by physiological agonists such as thrombin and collagen. Compounds such as $\mathrm{Ca}^{2+}$ ionophore [A23187, ionomycin], phorbol esters (9), complement protein C5b-9 $(69,229)$ as well as high shear stress $(230-233)$, contact with surfaces (234) and low temperatures (235) also are factors activating platelets. The nonphysiological agonists directly affect second messenger levels (9). The essential step of activation, which translates to the increase of intracellular calcium levels, affects intracellular enzymes (e.g. calpain which degrades actin-binding protein, talin and myosin) and protein kinase $C$ (PKC) (9). The activation of several isoforms of PKC follows the $\left[\mathrm{Ca}^{2+}\right]_{i}$ increase and phosphorylation of intracellular proteins involved in signal transduction and structure. The calcium increase is also caused by platelet activation by ADP or thrombin. Complement activation is a mechanism involved in PL-EV release which remains unaffected by activated enzymes (i.e. calpain). Another stadium of elevated PL-EV concentration is accompanied by fibrin association (236) and disintegration of platelets during and after thrombus formation (237) and implicates that the presence of PL-EVs in blood may reflect degradation of thrombus (9).

PL-EV formation also occurred in stored platelets undergoing senescence (14) or upon induction of apoptosis $(118,238)$. Although programmed cell death involves DNA fragmentation, a process which does not happen in anucleated cells, platelets contain apoptosis-associated proteins (9). The intrinsic pathway of apoptosis triggers caspase 3 formation and cleavage of structural proteins, such as ROCK I.

ROCK I participates in the phosphorylation of myosin light chain and induction of membrane contraction $(78,120)$.

However, there is another process leading to a vesicle production in damaged platelets, i.e. in platelets with a shorter life span. Higher levels of PL-EVs were found in patients with acquired clonal disorder of abnormal hematopoietic stem cells with deficiency in glycosyl-phospatidylinositol (GPI)-anchored proteins (paroxysmal nocturnal hemoglobinuria, PNH). Lack of these GPI-anchored proteins results in a deletion of the complement inhibitors CD55 and CD59 and implicates an increased risk of thrombosis development $(89,169)$.

\subsubsection{Clearance of PL-EVs}

In animals, upon introduction into blood circulation, PL-EV clearance takes only a few minutes, e.g. $30 \mathrm{~min}$ in mice, $10 \mathrm{~min}$ in rabbits $(11,239)$. PL-EV clearance in thrombocytopenic patients after transfusion of platelet concentrates happened with a half-life of 5.3 to 5.8 hours, depending on the subgroups of PL-EVs (228) and was much faster than the clearance of platelets from PCs. 
Several potential clearance pathways relate to enhanced EV-clearance. The consumption "by hemostatic events such as bleeding, thrombosis and clot formation" (1) is one of the clearance mechanisms. Moreover, the generally accepted mechanism involves PS exposure on PL-EVs (240). Beside "find-me" messages, PS exposure serves as the most common "eat-me" signal for phagocytic cells of the reticuloendothelial system (RES), as a sign of altered or pathologic cells and cell fragments (241). It is assumed, that there are multiple distinct PS receptors on phagocytic cells which are able to bind directly or indirectly to PS exposed on cells or cell fragments prior to being subjected to engulfment (240). PS-binding protein lactadherin (also known as milk fat globule-epidermal growth factor-8, MFG-E8), a macrophage-derived indirectly binding opsonin, is supposed to be involved in clearance of PL-EVs (242). Lactadherin-deficient mice have higher plasma levels of PL-EVs and the opsonization may occur in splenic macrophages from these mice. Patients with immune thrombocytopenic purpura (ITP) showed a higher PL-EV level after splenectomy than non-splenectomized ITP patients. However, significantly different results were only obtained for the elevated levels of red blood cell-derived and leukocyte-derived EVs in the splenectomized group (243). This research refers to an alternative pathway of PL-EV-clearance employing the binding of C3b complement on PL-EVs via complement receptor 1 (CR1, CD35) on erythrocytes in order to deliver PL-EVs to macrophages in liver and spleen (11).

Altogether, a different origin of PL-EVs, from resting and activated platelets or megakaryocytes, and rapid clearance mechanisms of PL-EVs let assume that PL-EV production is balanced under physiological and pathological conditions (226).

\subsubsection{Detection techniques}

Because of an increasing interest in the detection of extracellular vesicles, especially of the small exosome fraction, many new techniques have been developed. The classic and most widely used method in the detection of extracellular vesicles is conventional or standard flow cytometry (sd-FCM) $(9,218,244)$, with its ability to detect distinct EVs and their origin from mother cells using specific fluorescent labeled monoclonal antibodies (moAbs). A survey of the International Society of Thrombosis and Hemostasis (ISTH) indicated the use of FCM in $75 \%$ of the reports (245). Beside this method, new techniques are applicable to PL-EV measurement and two of them were used in this study, e.g. high-sensitivity flow cytometry (hs-FCM) (see page 59, chapter 4.6.3.3) and Nanoparticle Tracking Analysis (NTA; see page 60, chapter 4.7). However, it is worth notifying that the evaluation of an absolute particle count is still the greatest challenge (56). Three used techniques in this study with variable possibilities and limitations, which may be complementary, were used to quantify and to characterize PL-EVs from PCs. As mentioned earlier, PL-EVs include larger microvesicles and smaller exosomes; therefore, two new techniques were necessary for the analysis of EVs and EXs beyond conventional FCM. There are detection limits within these three techniques, as well as advantages and disadvantages of their application, as listed in Table I-7. 
Compared with sd-FCM, the hs-FCM indicated enhanced forward scatter (FS) resolution, a lower background noise and a 9-fold increase of the PL-EV count in PFP from the patient's plasma (246).

Table I-7: Pros and cons of detection techniques for measurement of extracellular vesicles

\begin{tabular}{|c|c|c|c|}
\hline & $\begin{array}{l}\text { sd-FCM } \\
\text { (i.e. Navios }{ }^{\mathrm{TM}} \text { ) }\end{array}$ & $\begin{array}{l}\text { hs-FCM } \\
\text { (i.e. A-50 Micro FCM) }\end{array}$ & $\begin{array}{l}\text { NTA } \\
\text { (i.e. NS500) }\end{array}$ \\
\hline $\begin{array}{l}\text { Lower detection } \\
\text { limit of beads }\end{array}$ & $\begin{array}{l}500 \mathrm{~nm}^{*} \\
\text { (polystyrene) }\end{array}$ & $\begin{array}{l}100 \mathrm{~nm}^{*} \\
\text { (polystyrene) }\end{array}$ & $50 \mathrm{~nm}$ \\
\hline $\begin{array}{l}\text { Lower detection } \\
\text { limit for EVs } \\
\left.\text { (bead-eq }^{\star *}\right)\end{array}$ & $\geq 300 \mathrm{~nm}$ & $\sim 180 \mathrm{~nm}$ & $\geq 50 \mathrm{~nm}$ \\
\hline $\begin{array}{l}\text { Specificity } \\
\text { (cellular origin) }\end{array}$ & $\begin{array}{l}+++ \\
\text { (moAbs) }\end{array}$ & $\begin{array}{l}+++ \\
\text { (moAbs) }\end{array}$ & $(+)$ \\
\hline Advantages & $\begin{array}{l}\text { established method; } \\
\text { widely used; } \\
\text { small sample volume }\end{array}$ & $\begin{array}{l}\text { volumetric sample } \\
\text { system gives absolute } \\
\text { count; } \\
\text { small sample volume }\end{array}$ & $\begin{array}{l}\text { fluorescence is } \\
\text { adjustable }\end{array}$ \\
\hline Disadvantages & $\begin{array}{l}\text { quantification only by } \\
\text { count beads; } \\
\text { no detection of EXs }\end{array}$ & $\begin{array}{l}\text { underestimation of } \\
\text { smaller MVs }\end{array}$ & $\begin{array}{l}\text { long capture time } \\
\text { during analysis; } \\
\text { photobleaching of } \\
\text { fluorophores; high } \\
\text { sample volume }\end{array}$ \\
\hline Practicability & +++ & +++ & ++ \\
\hline References & $\begin{array}{l}(246,247) ; \\
\text { by experience }\end{array}$ & $\begin{array}{l}(214,246) \\
\text { Manufacturer; personal } \\
\text { correspondence with O. } \\
\text { Kenyon; by experience }\end{array}$ & $\begin{array}{l}(9,248,249) \\
\text { by experience }\end{array}$ \\
\hline
\end{tabular}


In 2008, Robert et al. introduced a standardized protocol of PL-EV counting with the help of a newer FCM (FC500, Beckmann-Coulter) using fluorescent size-calibrated, polystyrene microspheres in order to apply the protocol to metacentric studies (218). However, the trial did not succeed because the data of PL-EV analysis by FCM are hardly comparable with one another as they are produced by different instruments within one investigation center.

Additionally, FCM protocols and the pre-analytical preparation of samples within one center differ from one another. In the literature, many data on PL-EV concentrations in plasma samples from healthy individuals were reported, as seen in Table I-8. Comparable data on PL-EV concentrations in PC samples are listed in Table I-9. A standard protocol for measurement of PL-EVs needs defined standard criteria for both, PL-EVs in blood samples and PL-EVs in PCs.

The following criteria of PL-EV analysis in blood samples by FCM were described: (i) Standardization of pre-analytical variables is necessary. This includes venipuncture (20- or 21gauge needle; antecubital vein; first 2 - $3 \mathrm{ml}$ should be discarded), collection tube (sodium citrate is most common), transportation (horizontal position), time to/ and differential centrifugation (stable within $2 \mathrm{~h}$ in citrated or CTAD tubes; cell-free plasma with 1,500 - 2,500xg for 15 - $20 \mathrm{~min}$ followed by $13,000 \mathrm{~g}$ for $2 \mathrm{~min})(250,251)$. (ii) The commonly used expression analysis of specific antigens such as GPIIb/IIla or GPIb with or without PS-expression needs to be defined (252). (iii) The definition of EV size from 0.1 to $1 \mu \mathrm{m}$ is necessary (218). (iv) Implementation of adequate calibration beads and the use of bead-equivalent size range according to physical differences between biological vesicles and latex or silica spheres would be of importance $(214,246)$, as shown in Table I-10.

In this context, depending on investigations of the real size distribution of biological vesicles by FCM using the Mie-theory and Rayleigh scattering, it has to be accepted that cell-derived vesicles correspond to the size of beads with an equivalent size and not to the measured bead size. "In theory, a $1.0 \mu \mathrm{m}$ sized platelet microparticle would scatter as much light as a polystyrene microsphere of about $0.5-0.6 \mu \mathrm{m}$ in diameter." "Our data indicate that a size gate based on 0.5 - $0.9 \mu \mathrm{m}$ polystyrene microspheres detects cellular particles of approx. 1 - $2 \mu \mathrm{m}$ of diameter on Apogee A40" (214). Chandler reported, thereby, the approx. 10-fold lower efficacy in light scattering of biological vesicles due to the lower refractive index which means, that the smallest biological vesicle must be larger than $~ 500 \mathrm{~nm}$ in order to be detected by FCM (49). In the view of the fact that the application of hs-FCM facilitates discrimination of different vesicle populations in size, new biological information may become more accessible (246). 
Table I-8: Designation of PL-EV concentrations from plasma samples of healthy individuals by flow cytometry as reported in the literature

\begin{tabular}{|c|c|c|c|}
\hline Flow cytometer & EV region set on beads & $\begin{array}{l}\text { Materials: } \\
\text { PPP, PFP, ND }\end{array}$ & References \\
\hline FC500 (BC) & $\begin{array}{l}>0.5 \mu \mathrm{m},<0.9 \mu \mathrm{m} \\
\text { (polystyrene) }\end{array}$ & $407-3039 / \mu \mathrm{L}$ & (218) \\
\hline Apogee A40 & $\begin{array}{l}<0.4 \mu \mathrm{m} \\
\text { (polystyrene) } \\
\leq 1 \mu \mathrm{m} \text { (cellular origin) }\end{array}$ & $6-300 \times 10^{9} / \mathrm{L}$ & (214) \\
\hline FACSAria (BD) & $\begin{array}{l}0.3 \mu \mathrm{m},<1 \mu \mathrm{m} \\
\text { (polymethyl-methacrylate) }\end{array}$ & $220 / \mu L^{*}, 420 / \mu L^{* *}$ & $(80)$ \\
\hline FACSCalibur (BD) & $\begin{array}{l}\text { unknown; } \\
\text { count beads: } 3 \mu \mathrm{m} \text { (latex) }\end{array}$ & $147 \pm 238 \times 10^{3} / \mathrm{mL}$ & (193) \\
\hline EPICS XL-MCL (BC) & $\begin{array}{l}0.1 \mu \mathrm{m}-6.0 \mu \mathrm{m} \\
\text { (polystyrene) }\end{array}$ & $\begin{array}{l}5,3 \times 10^{3} / \mathrm{mL} \\
\text { of whole blood } \dagger\end{array}$ & (253) \\
\hline$\Delta$ & ND & $\begin{array}{l}\leq 1000 / \mu L \\
=1 \text { PL-EV per } \sim 200 \\
\text { PLT }\end{array}$ & (254) \\
\hline$\Delta$ & ND & $100-4000 / \mu \mathrm{L}$ & (245) \\
\hline
\end{tabular}

PPP: platelet poor plasma; PFP: platelet free plasma; ${ }^{*}$ after ionophore; ${ }^{* *}$ after TRAP-6; tonly annexin V+ PL-EVs; in italic letter: sd-FCM; ND: not determined; $\Delta$ : not mentioned

Interestingly, Rank at al. reported that the PL-EV count (MPs positive for CD61-labeling) from PCs reached approximately $10000 \times 10^{6} / \mathrm{L}$ on the first day. The level of PL-EVs decreased during storage and amounted to approximately $7500 \times 10^{6} / L$ on the fifth day. These results referred to measurement of all cell-derived vesicles by FACScan (Becton Dickinson) according to Berckmans (86) formula which is as follows:

number/ $L=$ multiplication of several dilution steps $x$ used sample volume and the analyzed volume (15).

However, it is not only a challenge to measure size but also to measure concentration of vesicles. EV analysis has still to be improved. This necessity is supported by the "iceberg theory" (49) and theory of the "swarm detection" (255) which imply that the FCM technique significantly reduces the values of EVs due to not only limited detection technique of commonly 
used FCM but also due to the unexpected detection of large numbers of smaller vesicles, the "swarm".

Table I-9: PL-EV concentration of platelet concentrate samples measured by flow cytometry as reported in literature

\begin{tabular}{|c|c|c|c|}
\hline $\begin{array}{l}\text { Materials: } \\
\text { PRP, PPP, PFP }\end{array}$ & Machines & Concentration & References \\
\hline $\begin{array}{l}\text { Buffy coat-derived } \\
\text { platelet concentrates; } \\
\text { PFP }\end{array}$ & A-50 (Apogee) & $\begin{array}{l}24,2 \times 10^{3} / \mu \mathrm{L} \\
\equiv 24200 \times 10^{6} / \mathrm{L} \\
\text { CD42b (+streptavidin-AF } \\
647 \mathrm{~nm}-\mathrm{R}-\mathrm{PE})\end{array}$ & (256) \\
\hline $\begin{array}{l}\text { Plateletpheresis } \\
\text { concentrates; } \\
\text { PPP }\end{array}$ & ND & $\begin{array}{l}1264 \times 10^{6} / \mathrm{L} \\
\text { CD42b+ } \\
\text { (1\% paraformaldehyd } \\
\text { in PBS) }\end{array}$ & (257) \\
\hline $\begin{array}{l}\text { Plateletpheresis } \\
\text { concentrates; } \\
\text { after freeze: PFP } \\
\text { (on day 1) }\end{array}$ & FACScan (BD) & $\begin{array}{l}10000 \times 10^{6} / \mathrm{L} \\
\mathrm{CD} 61+\end{array}$ & (15) \\
\hline $\begin{array}{l}\text { Buffy coat-derived PCs; } \\
\text { lonophore-stimulated } \\
\text { PRP (on day 1) }\end{array}$ & FACScalibur (BD) & $\begin{array}{l}\text { PL-EV ratio: } \\
\text { approx. } 5 \%\end{array}$ & (258) \\
\hline
\end{tabular}

Using NTA, Harrison et al. and Dragovic et al. suggested that $90 \%$ of EVs in PFP are below $200 \mathrm{~nm}$ of a concentration of 1 to $5 \times 10^{10} / \mathrm{mL}$ (lipoproteins excluded) $(248,255)$. In this aspect, measured PL-EV concentrations by FCM become rather estimated values. In addition, the recent literature provides numerous comments on the NTA system. As seen in Table I-10, the material of calibration beads is the most important variable in size-detection by NTA and FCM. FCM is not the only method, which is based on the refractive index of vesicles scattering light. Therefore, more suitable material for standardization of EV measurement, such as silica beads, was preferred $(214,259)$. 
Table I-10: Bead and vesicle characteristics for detection by light scatter

\begin{tabular}{l|ccc}
\hline & $\begin{array}{l}\text { Polystyrene } \\
\text { beads }\end{array}$ & Silica beads & $\begin{array}{l}\text { Organic vesicles } \\
\text { (membrane and } \\
\text { lipid vesicle) }\end{array}$ \\
\hline $\begin{array}{l}\text { Refractive index } \\
\text { (RI; } \boldsymbol{\eta})\end{array}$ & 1.59 & $1.43-1.46$ & 1.39 \\
$\begin{array}{l}\text { Measured amount of scattered } \\
\text { light by NTA (in ratio) }\end{array}$ & 2.57 & 1.5 & 1 \\
$\begin{array}{l}\text { RI applying the Rayleigh } \\
\text { approximation }\end{array}$ & - & - & 1.41 \\
$\begin{array}{l}\text { Predicted scattering ratio by the } \\
\text { Rayleigh approximation }\end{array}$ & 3.16 & & 1 \\
\hline $\begin{array}{l}\text { (Data from (259)) } \\
\text { (25) }\end{array}$ & & & \\
\hline
\end{tabular}

Using the Rayleigh approximation, consistent values of the same refractive indices of polystyrene spheres, silica spheres and EVs were achieved together with comparable values of both EVs and silica beads. Although, silica beads and EVs scatter similar amount of light, EVs may be undervalued as compared to silica beads (259). Gardiner et al. obtained accurate measurements of $60 \mathrm{~nm}$ silica microspheres and provided a very important advice, that the "minimum track length has no effect on concentration measurement but determines the proportion of events used to build a size distribution". In the course of measurement of Cell Mask-labeled EVs from plasma, the fluorescent measurement was consistent to the light scattering size distribution of cell derived vesicles (259). Nonetheless, the phenomenon of photobleaching of fluorophores, the most commonly used to identify cell lineage specificity of EVs, constitutes a serious problem for fluorescence measurement by NTA. Indeed, body fluids or samples from blood concentrates are polydisperse for vesicles and not user-friendly. Gardiner et al. also attended care of overestimating larger particles due to the change of size distribution with different camera levels, especially in samples with very low EV numbers. The problem of analyzing polydisperse samples by NTA deals with different requirement for light intensity of the beam for particles of different sizes to scatter the same light. In order to detect smaller vesicles, usually higher camera levels are needed as compared to larger vesicles. Either one sample has to be measured under three distinct settings (low dilution and low camera level for large EVs; medium dilution and middle camera level for microvesicles; high dilution and high camera level for exosomes) or the larger vesicle has to be diluted so that only small EVs are analyzed (259). 
Additionally, the high amount of the EV-typical sized lipoprotein particles (over 98\%) in plateletfree plasma (PFP) of healthy individuals was demonstrated. At least, the pellet of ultracentrifuged PFP contained $0.5-5.0 \times 10^{10} \mathrm{EV} / \mathrm{mL}$ of an average size of $70-120 \mathrm{~nm}$. 


\section{Aims of the study}

During storage of plateletpheresis concentrates (PC) under blood banking conditions for up to 4 days, platelets develop so-called platelet storage lesion (PLS), which is a major limitation of in vitro platelet storage. Previous investigations have shown that platelet-derived extracellular vesicles (PL-EVs) are a part of PLS. The current approaches to quality control (QC) of PCs from platelet hemapheresis now include the analysis of PL-EVs performed at the University Hospital of Regensburg, as presented in Figure II-1.

The primary aim of this dissertation was to investigate how the amount of PL-EVs from PCs may contribute to the quality-defining process within QC program. The study includes the determination of a correlation between the measurement of PL-EVs in fresh PCs and PCs over time and the measurement of well-established platelet function tests, i.e. expression of CD62P on platelets' surface after activation with thrombin receptor activating peptide 6 (TRAP-6).

The first goal was to validate the method of PL-EV analysis by standard flow cytometry (sdFCM). The two new analytical methods, the high-sensitivity flow cytometry (hs-FCM) and nanoparticle tracking analysis (NTA) were compared to sd-FCM (Inter-assay validation). Further, it was aimed to show, that aggregometry with PC-samples is not the method of choice for $\mathrm{QC}$, since aggregometry required the whole $\mathrm{PC}$ and it does not allow a separate analysis of individual PC components (i.e. platelets, PL-EVs and surrounding plasma). Not only coagulability, number and amount of PL-EVs was detected, it was also questionable, if several conditions of PCs, such as preparation, sampling, storage or activation may affect the attendance of platelet vesiculation, as described in chapter $V 5.1$ of results.

The correlation among donor-specific parameters and laboratory parameters and qualitydefining parameters of PCs was investigated, because donor-specific parameters (i.e. body mass index, etc.), collection-specific parameters (i.e. time of apheresis) and storage-associated parameters (i.e. shear stress) may also influence the quality of PCs, as seen in chapter V 5.2.

Three methods of PL-EV analysis, i.e. sd-FCM, hs-FCM and NTA were correlated to the standard platelet function test (CD62P expression). Changes of PL-EV amount over time during storage was determined, which may correlate with standard quality-defining laboratory tests as well as with extra conditions, i.e. irradiation, as described in chapter V 5.3.

Investigations was necessary in order to evaluate the amount of EVs originating from other cells than from platelets within PCs (as described in chapter V 5.4), because that amount may affect the total number, size and function of cell-derived vesicles in blood components, in this case PCs. 
In vitro analysis of platelet storage lesion (PSL) in platelet concentrates (PCs)

\section{Plasma}

Protein degradation

Enzyme effects

\section{Platelets (PLTs)}

- Shape change
- activation

\section{Methods for validation:}

I. Flow cytometry:

TRAP6-assay

II.
(e.g. Born)

\section{Principle:}
Batch analysis of PLTs and PL-EVs

\section{Principle:}

Separate analysis of PLTs and

PL-EVs; PLT stimulation with TRAP-6 (CD62P expression)
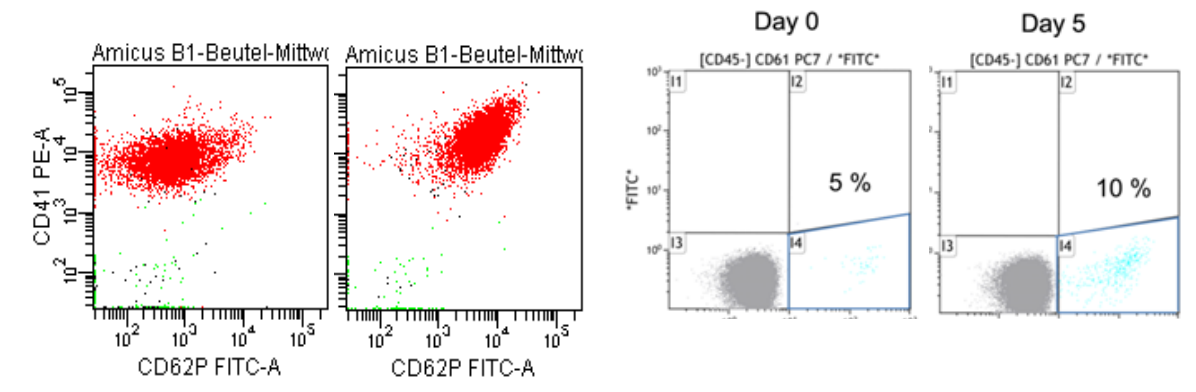

1. Validation of gated PLTs separated from PL-EVs

2. Analysis of PL-EV content during storage

\section{Aggregometry does not reflect specific platelet activation}

(formerly microparticles)

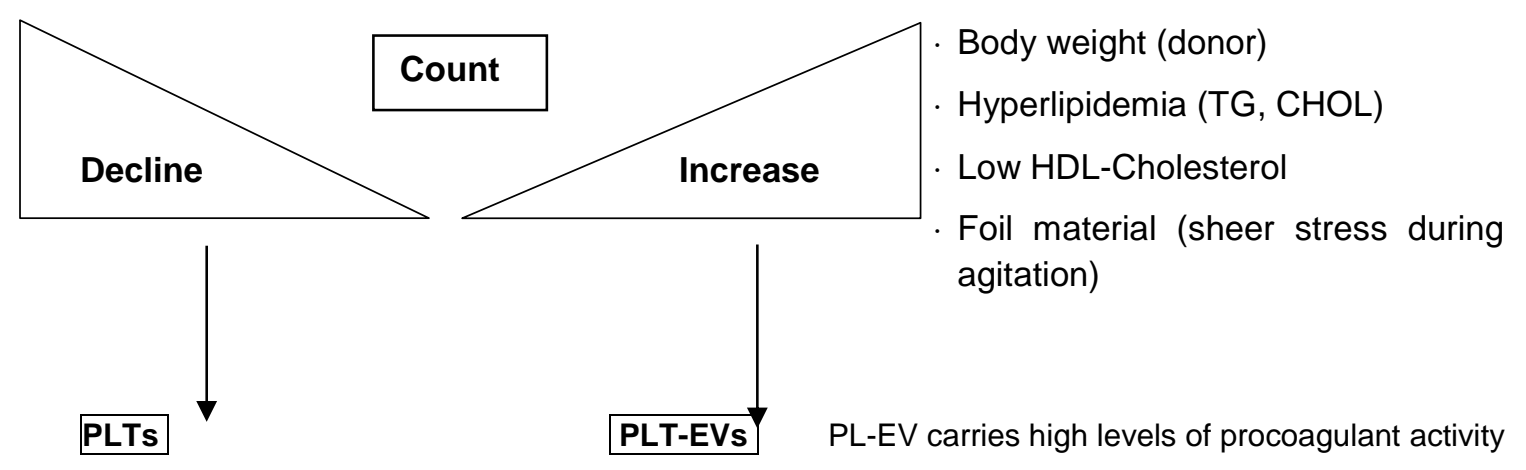

\section{Platelet-derived extracellular vesicles (PL-EVs)}

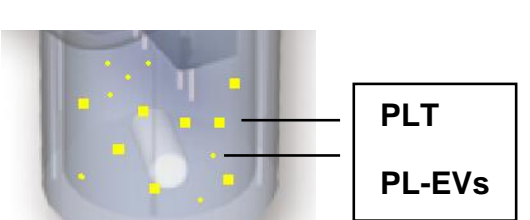

due to the artificial procoagulant activity of PL-EVs

Figure II-1: Investigation of quality in plateletpheresis concentrates in vitro involves the analysis of separate concentrate components, i.e. plasma, platelets and platelet-derived extracellular vesicles (PL-EVs) 
III Materials

\subsection{Technical equipment}

Machine Manufacturer

A50-Micro Flow Cytometer

ABL 90 FLEX

Amicus Crescendo Cell separator

Apact 4S Plus

BCS $\AA$ System

BD FACS Canto ${ }^{\mathrm{TM}} \|$

Blood bank refrigerator BL-520

BN ProSpec

Cobas Integra

Donor Tube Stripper

Fridge, $261844\left(2^{\circ} \mathrm{C}-8^{\circ} \mathrm{C}\right)$

Hematron $^{\mathrm{TM}} 3$

Heraeus Megafuge 1,0 R

Immulite $2000 \mathrm{XPi}$

Irradiation device Type IBL-437

Lamina Air Flow

Multiplate $\AA$ Analyzer

NanoSight NS500

Navios $^{\mathrm{TM}}$, AN 41158

Platelet Agitator PF96i

Platelet Agitator PFS84

Platelet Incubator PC3200h

Pro BP 3400

Reference Pipette 0,5-10 $\mu \mathrm{L}, 500 \mu \mathrm{L}$, 50-200 $\mu \mathrm{L}, 1000 \mu \mathrm{L}, 20 \mu \mathrm{L}, 50 \mu \mathrm{L}$

Research Pipette 10-100 $\mu \mathrm{L}, 100-1000 \mu \mathrm{L}$
Apogee Flow Systems Ltd., UK

Radiometer $\mathrm{GmbH}$, Willich

Fenwal Inc., Lake Zürich, IL, USA

Rolf Greiner Biochemica, Flacht

Siemens Healthcare Diagnostics

Becton Dickinson (BD-Bioscience),

Heidelberg

Philipp Kirsch GmbH, Offenburg, Germany

Siemens Healthcare Diagnostics

Roche Diagnostics, Germany

Fenwal Inc., Lake Zürich, IL, USA

Liebherr, Germany

Fenwal Inc., Lake Zürich, IL, USA

DJB Labcare Ltd, UK

Siemens Healthcare Diagnostics

Isotopen Diagnostik CIS GmbH, Dreieich

Thermo Electron Company

Dynabyte $\mathrm{GmbH}$, Munich, Germany

NanoSight Ltd., Amesbury, UK

Beckman Coulter GmbH, Krefeld

Helmer Laboratories, Noblesville, USA

Helmer Laboratories, Noblesville, USA

Helmer, Laboratories, Noblesville, USA

Welch Allyn Inc., NY

Eppendorf, Germany

Eppendorf, Germany 
continuation of table of technical equipment

\section{Machine}

Scale VC $3 \mathrm{~K} 1 \mathrm{M}$

Sunrise ${ }^{\mathrm{TM}}$ Tecan Photometer

ThermoScan Braun Welch Allyn

Trima-Accel® Cellseperator

TSCD-II Terumo

Ultracentrifuge SW-41TI rotor

Vista Dimension® 1500

Vortex-Genie 2

XE-5000 hematology analyzer

\section{Manufacturer}

Gottl/ Kern \& Sohn GmbH, Germany

Tecan Deutschland GmbH, Crailsheim

Kaz Europe SA

Terumo BCT Inc., USA

Terumo, Germany

Beckman, Fullerton, CA

Siemens Healthcare Diagnostics

USA Scientific Inc., USA

Sysmex, Norderstedt, Germany

\subsection{Consumables}

\section{Product}

Blood Gas Monovette®

Capillary tubes

Falcon tubes, $5 \mathrm{ml}$

Multiplate-disposable-Measuring cell

Pipette tip „epTIPS“ 0,1 - $2500 \mu \mathrm{L}$

Platelet Apheresis Kit

Platelet Apheresis Kit

Polypropylene round bottom tubes

$5 \mathrm{~mL}, 2 \mathrm{~mL}$

Polystyrene round bottom tubes, $5 \mathrm{~mL}$

Rad-Sure Blood irradiation indicator

Safety-Multifly-Set, 20G, 0,9 x 19mm

S-Monovette ${ }^{\circledR}$, Clotting activator

S-Monovette®, EDTA K 3

\section{Manufacturer}

Sarstedt, Germany

Sysmex GmbH, Germany

Sarstedt, Germany

Dynabyte GmbH, Münich, Germany

Eppendorf, Germany

Terumo BCT, Europe n.V. Belgium

Fenwal Inc., Lake Zürich, IL, USA

Sarstedt, Germany

Falcon/BD, Eremdodegem, Belgium

LMB Technologie GmbH, Schwaig

Sarstedt, Germany

Sarstedt, Germany

Sarstedt, Germany 
continuation of table of consumables

Product

S-Monovette $\AA$, Sodium-Citrat

Spiking Mandrel R4R1401

Syringe without Luer Look $20 \mathrm{~mL}, 50 \mathrm{~mL}$

Transfer-needle, 27G

\section{Manufacturer}

Sarstedt, Germany

Fenwal Inc., Lake Zürich, IL, USA

Falcon/BD, Eremdodegem, Belgium

Sarstedt, Germany

\subsection{Reagents}

\section{Substance}

ACD-A, RDB8652

ADP (adenosine diphosphate), $5 \mu \mathrm{M}$

Apolipoprotein A-I Flex® reagent cartridge

Apolipoprotein B Flex® reagent cartridge

Apolipoprotein CI Human ELISA Kit

Apolipoprotein J Human ELISA Kit

Aqua dest.

Arachidonic acid

BC-Thrombin-Reagent

Berichrom-PAI 1 Kit

C3-Complement Flex® reagent cartridge

$\mathrm{CaCl}_{2}$ Solution

Calcium Flex® reagent cartridge

Calibration BeadMix \#1493

Calibration beads, $100 \mathrm{~nm}$

Calibration beads, $100 \mathrm{~nm}$, green fluorescent

Calibration beads, $200 \mathrm{~nm}$

Calibration beads, $50 \mathrm{~nm}$

CD41-PE, Clone P2, Type IgG 1

\section{Manufacturers}

Fenwal Inc., Lake Zürich, IL, USA

Rolf Greiner Biochemica

Siemens Healthcare Diagnostics

Siemens Healthcare Diagnostics

Abcam plc., Cambridge, UK

BioVendor $\mathrm{GmbH}$, Heidelberg

B. Braun, Melsungen

Rolf Greiner Biochemica

Siemens Healthcare Diagnostics

Siemens Healthcare Diagnostics

Siemens Healthcare Diagnostics

Siemens Healthcare Diagnostics

Siemens Healthcare Diagnostics

Apogee Flow Systems Ltd, UK

NanoSight Ltd., Amesbury, UK

NanoSight Ltd., Amesbury, UK

NanoSight Ltd., Amesbury, UK

NanoSight Ltd., Amesbury, UK

IOTest $\AA /$ Beckman Coulter 
continuation of table of reagents

\section{Substance}

CD61-PC7, Clone SZ21, Type IgG 1

CD61-PerCP, Clone RUU-PL 7F12, Type

$\operatorname{lgG}_{1}-$ Kappa

CD62P-FITC, Clon CLB-Throm/6, Type IgG 1

Cellpack (PK-30L, 20 L)

Cholesterol Flex® reagent cartridge

Collagen

C-reactive Protein Flex® reagent cartridge

ELISA Kit Human Apo-B48

Fibrinogen N Antiserum

Glucose Flex® reagent cartridge

Hemoglobin A1c Kit

High-Density-Lipoprotein-Cholesterol Flex®

reagent cartridge

Human-Apolipoprotein E Antiserum

IgG1-PC7, Clone 679.1Mc7, isotypic control

Immulite IGF-BP-3 Test

Immulite IGF-1 Test

Kalium Flex® reagent cartridge

Low-Density-Lipoprotein-Cholesterol Flex®

reagent cartridge

MegaMix

Multifibren U

$\mathrm{NaCl}(0.9 \%)$, HAGE1324

$\mathrm{NaCl}, 0.9 \%$

N-Latex Lp(a)

N-Lp(a) Control SY

N-Lp(a) Standard

Optiprep Density Gradient Medium

\section{Manufacturer}

IOTest $₫ /$ Beckman Coulter

BD- Biosciences

IOTest $\circledast$ / Beckman Coulter

Sysmex GmbH, Germany

Siemens Healthcare Diagnostics

Rolf Greiner Biochemica

Siemens Healthcare Diagnostics

Tecan Deutschland GmbH

Siemens Healthcare Diagnostics

Siemens Healthcare Diagnostics

Roche Diagnostics

Siemens Healthcare Diagnostics

Siemens Healthcare Diagnostics

IOTest®/ Beckman Coulter

Siemens Healthcare Diagnostics

Siemens Healthcare Diagnostics

Siemens Healthcare Diagnostics

Siemens Healthcare Diagnostics

BioCytex, Marseille, France

Siemens Healthcare Diagnostics

Baxter, Germany

B. Braun Melsungen AG, Germany

Siemens Healthcare Diagnostics

Siemens Healthcare Diagnostics

Siemens Healthcare Diagnostics

Sigma-Aldrich, Steinheim, Germany 
continuation of table of reagents

\section{Substance}

PBS-Dulbecco, w/o Ca ${ }^{2+} / \mathrm{Mg}^{2+}$ Buffer

Ristocetin

TRAP-6 (Thrombin-Receptor-Activating-

Peptide, SFLLRN), $1 \mu \mathrm{M}$

Triglyceride Flex® reagent cartridge

Tyrodes buffer

\section{Manufacturer}

Biochrom AG, Berlin

Rolf Greiner Biochemica

Bachem Biochemica, Heidelberg

Siemens Healthcare Diagnostics

All components are acquired from Sigma-

Aldrich, Steinheim, Germany

\subsection{Analytical Software}

Software

Amicus Crescendo Software V 3.2

Apogee Histogram Software, V3-1

FACSDiva Software, Version 6.1.3

Multiplate Software Version V 2.02.11

NanoSight NTA 2.3 Analytical Software

Navios Software, Version 1.0

Office 2012 Office suite

Reference Manager

SigmaPlot 12

SPSS 20 statistical software package

swisslab, Release 2011_03

Trima-Accel®, Software 5.1

XLSTAT MS Excel

\section{Manufacturer}

Fenwal Inc., Lake Zürich, IL, USA

Apogee Flow Systems Ltd, UK

Becton Dickinson (BD-Bioscience),

Heidelberg

Dynabyte medical, Munich

NanoSight Ltd., Amesbury, UK

Beckman Coulter GmbH, Krefeld

Microsoft, Unterschleißheim

Thomson Reuters, San Francisco, USA

Systat Software GmbH, Erkrath

IBM, Ehningen

Roche Deutschland Holding GmbH

Terumo BCT Inc., USA

Microsoft, Unterschleißheim 


\subsection{Preparation of solutions}

\subsubsection{TRAP-6}

TRAP-6 (Thrombin-Receptor-Activating-Peptide, SFLLRN) stock solution, $3 \mu \mathrm{M}$ :

- $5 \mathrm{mg}$ TRAP-6 dissolved in $2225 \mu \mathrm{L}$ Aqua dest. (3 mM stock solution 1)

- $100 \mu \mathrm{L}$ stock solution 1 diluted in 9,9 mL PBS-Dulbecco (30 $\mu \mathrm{M}$ stock solution)

- Aliquots á $20 \mu \mathrm{L}$ stored in cups with screw-cap (Eppendorf) at $-15^{\circ} \mathrm{C}$ or at lower temperatures are stable for one year according to the manufacturers recommendation

- Before utilization, aliquots have to be thawed at room temperature for a few minutes (min)

\subsubsection{Tyrode buffer}

Calcium-free Tyrode-Hepes buffer or modified Typrode-Hepes buffer (Tyrode- $\mathrm{N}-2$ hydroxyethylpiperazine- $N$-2-ethanesulfonic acid), $\mathrm{pH} 7.4$, sterile-filtrated

$\begin{array}{lllll}138 & \mathrm{mM} & \mathrm{NaCl} & \mathrm{MW}: 58.442 & 8.065 \mathrm{~g} / \mathrm{L} \\ 3 & \mathrm{mM} & \mathrm{KCl} & \mathrm{MW}: 74.56 & 0.224 \mathrm{~g} / \mathrm{L} \\ 12 & \mathrm{mM} & \mathrm{NaHCO}_{3} & \mathrm{MW}: 84.01 & 1.008 \mathrm{~g} / \mathrm{L} \\ (0.4 & \mathrm{mM} & \left.\mathrm{NaH}_{2} \mathrm{PO}_{4}\right) & \mathrm{MW}: 137.99 & 0.055 \mathrm{~g} / \mathrm{L} \\ 1 & \mathrm{mM} & \mathrm{MgCl}_{2} & \mathrm{MW}: 203.3 & 0.203 \mathrm{~g} / \mathrm{L} \\ 5 & \mathrm{mM} & \text { glucose } & \mathrm{MW}: 180.2 & 0.901 \mathrm{~g} / \mathrm{L} \\ 10 & \mathrm{mM} & \text { Hepes } & \mathrm{MW}: 238.306 & 2.383 \mathrm{~g} / \mathrm{L} \\ 10 & \mathrm{mM} & \text { EDTA } & \mathrm{MW}: 372.2 & 3.722 \mathrm{~g} / \mathrm{L}\end{array}$

\subsection{Plateletpheresis concentrates}

Hemapheresis donations for platelet concentrates (PCs) were obtained according to the German regulations of Bundesärztekammer (33) for blood donation from healthy volunteer platelet apheresis donors. All donors gave informed consent. For a single-donor apheresis cell separators Trima Accel ${ }^{\circledR}$ (Terumo BCT, with the single-needle-System) and Amicus (Fenwal Inc., with the double-needle-System) were used under standard procedures of blood donation centers. As specified in the manufacturing authorization (PEI.H.03019.01.1, "HumanThrombozytapherese-konzentrat UKR, leukozytendepletiert, bestrahlt"; PEI.H.02563.01.1, "Human-Thrombozyten-Apheresekonzentrat UKR, leukozytendepletiert"), PCs included human platelets from a single donation in amount varying from 2.0 to $4.0 \times 10^{11}$ platelets/ unit in a total volume varying from $200 \mathrm{~mL}$ to $300 \mathrm{~mL}$, whereas leukocyte content was lower than $1 \times 10^{6} /$ unit and erythrocyte content lower than $3 \times 10 \%$ unit.

Further components are autologous plasma $(0.74-0.85 \mathrm{~mL} / \mathrm{mL})$ and Anticoagulant Citrat Dextrose Solution Formula A (ACD-A; Fenwal Inc.; 0.15 - $0.26 \mathrm{~mL} / \mathrm{mL}$ ). 
Gamma irradiation with 25 Gy (Irradiation device Type IBL-437, Isotopen Diagnostik CIS GmbH, Dreieich) of PCs was conducted in our laboratory on the day of platelet collection (day 0 ). Irradiation was successful when the 'NOT' in the window of Rad-Sure indicator darkened after irradiation and the indicator: 'IRRADIATED' was displayed. Platelet concentrates were stored for up to 7 days at $22^{\circ} \mathrm{C} \pm 2^{\circ} \mathrm{C}$ under constant agitation at $60 \mathrm{cycles} / \mathrm{min}$ (Platelet Agitator PF96i, Helmer Laboratories, Noblesville, USA).

\subsection{Red blood cell units}

For analysis of vesicles in red blood cell (RBC) units, five expired and non-irradiated units were used. They were labeled as "Human-Erythrozytenkonzentrat CPD-SAGM L BSD/BRK" of the blood donation center "Bayerisches Rotes Kreuz, GmbH München" and stored under standard blood banking conditions at $4^{\circ} \mathrm{C} \pm 2^{\circ} \mathrm{C}$ (Blood bank refrigerator BL-520 according to DIN 58371, Philipp Kirsch $\mathrm{GmbH}$, Offenburg, Germany). The number of days exceeding the expiry date of RBC units varied from one to three days, i.e. red blood cells reached the age from 43 days up to 45 days. According to the manufacturer information, the standard RBC unit of a volume of 280 up to $380 \mathrm{~mL}$ comprises erythrocytes of a hematocrit level ranging from 0.5 to 0.7 and a stabilizing solution CPD (sodium citrate, citric acid, monohydric glucose, sodium-hydrogen phosphates di-hydrates). Additionally, RBC units contain an additive solution SAG-M (sodium chloride, adenine, glucose monohydrate, and mannitol) and donor plasma with residual leukocytes amounting to less than $1 \times 10^{6}$ per standard container.

\subsection{Blood samples of healthy donors}

Peripheral venous blood samples were collected on day 0 directly before apheresis. Test tubes were filled with ethylenediaminetetraacetic acid $\left(1.6 \mathrm{mg}\right.$ EDTA/ $1 \mathrm{~mL}$ blood; S-Monovette ${ }^{\circ}$, EDTA $K_{3}$, Sarstedt), or citrate (pre-dosed as a 0.106 molar solution, equivalent to $3.2 \%$ trisodium citrate; S-Monovette $\AA$, Sodium-Citrat, Sarstedt) in a mixed ratio of 1:10 (1 part citrate + 9 parts blood) or with beads coated with a clotting activator (silicate; S-Monovette ${ }^{\circledR}$, clotting activator, Sarstedt). Serum was obtained by centrifugation of blood samples at $2500 \mathrm{~g}$ for 15 min. To quantify apolipoprotein C-I, clusterin, apolipoprotein B48, insulin-like growth factor 1 (IGF-1), insulin-like growth factor binding protein 3 (IGFBP-3), samples were immediately stored at $2^{\circ}-8^{\circ} \mathrm{C}$ until measurements were carried out. All analytical techniques, except vesicle analysis, were implemented in the routine workflow at the Institute for Laboratory and Transfusion Medicine, University of Regensburg, Germany. 


\section{Methods}

\subsection{Background}

Altogether, 106 platelet concentrates have been investigated. Donor specific parameters (BMI, post-prandial status, blood pressure, etc.) were determined directly before platelet hemapheresis. Plasma parameters were determined by photometry, nephelometry, ELISA, CLIA, coagulometry or chromogenic method on clinical chemistry analyzers according to standard procedures of manufacturer.

PCs were exposed to effects of agonists of platelet activation and aggregometry of PCs was measured with the help of an impedance aggregometer (Multiplate $\AA$ ) and light transmission aggregometer (Born) according to the instructions of the manufacturer.

Analysis of platelet-derived extracellular vesicles (PL-EVs) was carried out by standard flow cytometry in volume-defined PC samples using a size calibrator micro bead cocktail (Megamix $\AA$ ). Additionally, PL-EVs were measured with a micro flow cytometer and partially fluorescent beads smaller sized as the MegaMix beads (Apogee bead mix \#1493). Concentration and allover particle size distribution of all plasmatic vesicles without immunostaining were analyzed by nanoparticle tracking analysis (NTA, NanoSight, UK). Platelet function tests were performed with an agonist of platelet activation by standard flow cytometry and platelet specific immunostaining.

For comparison, erythrocyte- and leukocyte-derived vesicles from PCs were also measured by flow cytometry. 


\subsection{Flow chart of plateletpheresis and sampling for quality control}

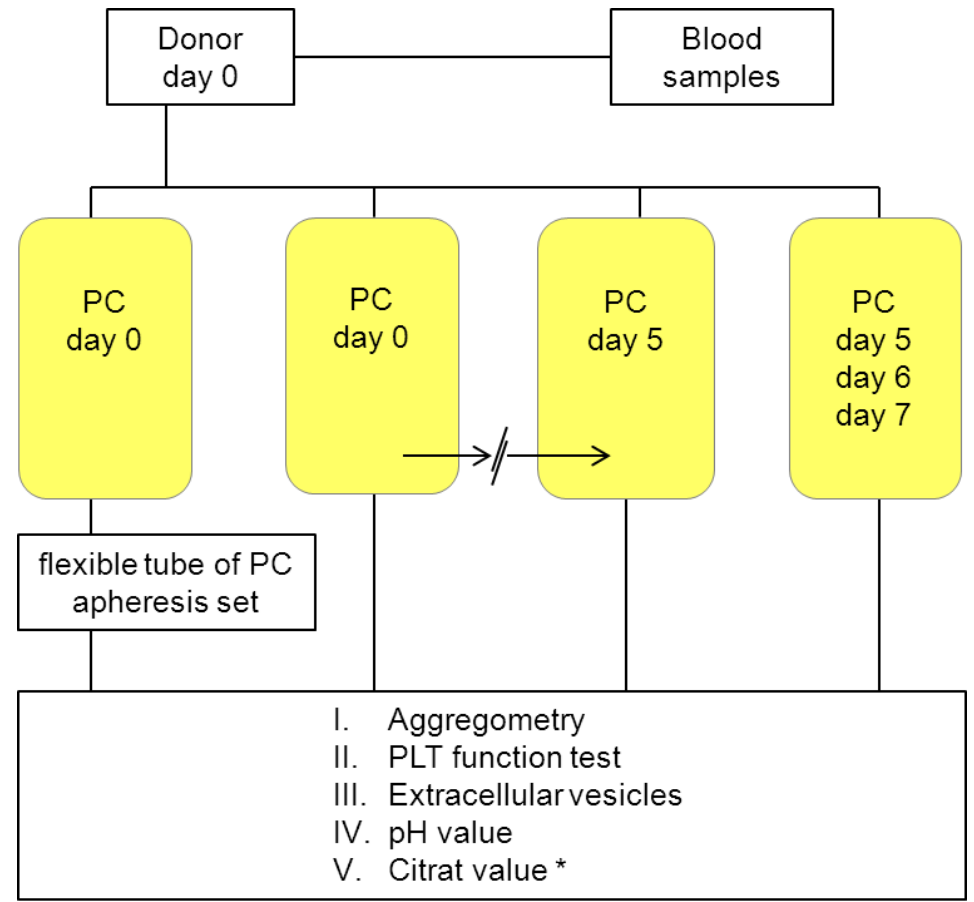

Figure IV-1: Workflow diagram for manufacturing and sampling of platelet concentrates (PC) on several days after apheresis

${ }^{*}$ Citrate values were measured merely on day 0 of PCs.

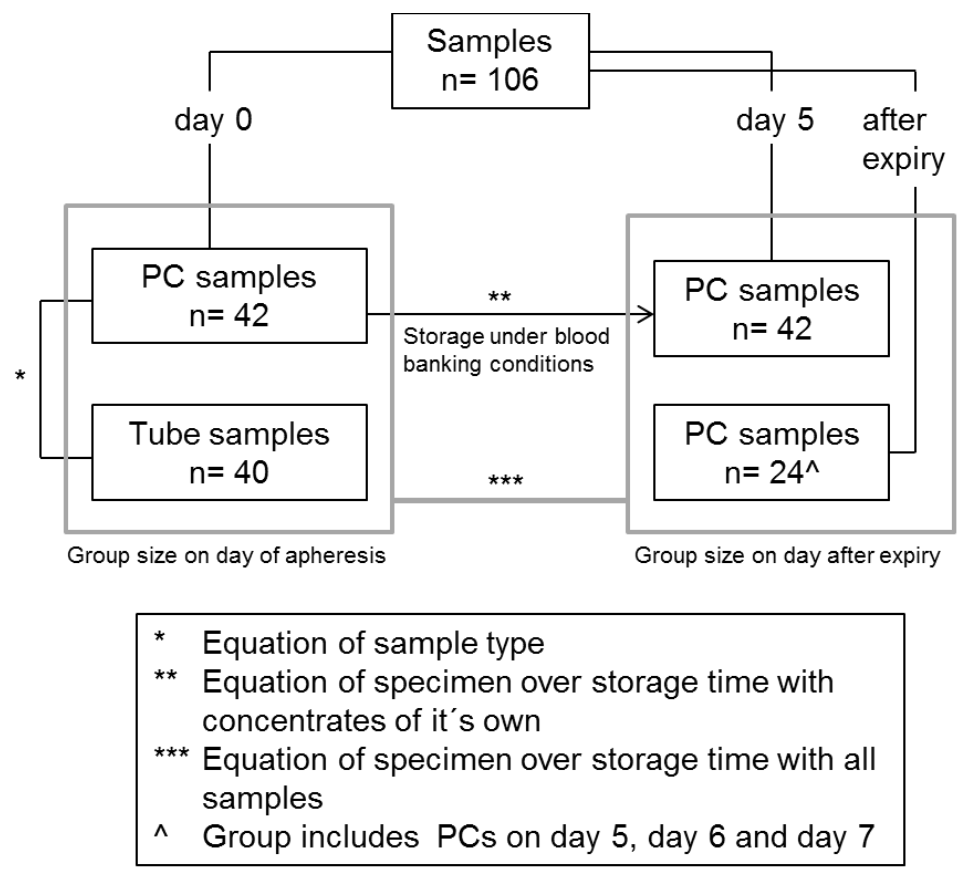

Figure IV-2: Flowchart of sample count $(\mathrm{n}=)$ from plateletpheresis concentrates

Overall, 106 PCs were analyzed. The first group included 42 PCs analyzed on day 0 and subsequently on day 5 . In the second group, 40 PC tube samples were analyzed exclusively on the day of hemapheresis (day 0 ) and in the third group 24 PCs after expiry date. 


\subsection{Sampling of platelet concentrates}

Tests for platelet (PLT) count of PCs, PLT function, pH-value, PL-EV amount and citrate values were performed on samples taken on day 0 and/ or day 5 , at day 6 , or day 7 after donation. Sampling of PCs on day 0 was performed before agitation and incubation at room temperature, sampling on day 5, 6 and 7 was carried out at room temperature after constant agitation of PCs incubated at $22^{\circ} \mathrm{C} \pm 2^{\circ} \mathrm{C}$ according to standard blood banking conditions.

Two different sample types of PCs after apheresis were used. At first, samples were drawn from the content of $\mathrm{PC}$ bags by sterile docking of spiking mandrel after thorough mixing of the bags. Next, samples were drawn from the flexible, concentrate-filled, tube system of the closed apheresis set after stripping the tube twice. Sampling by sterile docking is favorable in aspect of an arbitrary sample volume for tests of PC quality whereas the tube volume of the apheresis sets is limited. The PLT count in both different sample types was equal and amounted approximately $1,000,000,000 \mathrm{PLT} / \mathrm{mL}$.

While sampling, the PC bag concentrates were placed under a laminar flow hood and were connected with a spiking mandrel (R4R1401, Fenwal) by sterile docking immediately after opening the PC bag on injection port. The mandrel was tapped with a needle (Safety-MultiflySet, $20 \mathrm{G}$, and $0.9 \times 19 \mathrm{~mm}$, Sarstedt, Germany) to transport the content of the bag into the previously prepared test tubes.

In case of laminar flow hood conditions were not available, sampling from PC bags was performed by using a container closed to the apheresis set. The container was filled with PC content (the container was gently squeezed with two fingers) and welded with a heat sealer (TSCD-II Terumo, Germany) before the content was conveyed in the prepared tests tubes.

PC sample from a concentrate-filled, flexible tube connected with apheresis set was disconnected with a sterile heat sealer (TSCD-II Terumo, Germany). Perforation of the tube with a needle (Safety-Multifly-Set, 20 G, $0.9 \times 19$ mm, Sarstedt, Germany) under laminar airflow hood was performed to transfer the content of the tube into a prepared test tube. Flexible tubes of a length up to $28 \mathrm{~cm}$ and a radius of $0.15 \mathrm{~cm}$ gave a sample volume of max. $1.979 \mathrm{~mL}$, as determined with the help of the formula: $V=h \times \pi \times r^{2}\left(V=28 \mathrm{~cm} \times 3.1416 \times(0.15 \mathrm{~cm})^{2}=1.979\right.$ $\mathrm{cm}^{3}=1.979 \mathrm{~mL}$ ). Therefore, a tube of $1 \mathrm{~cm}^{3}$ volume corresponds to a sample of $1 \mathrm{~mL}$ and tubes with a length up to $1 \mathrm{~cm}$ bring about a sample volume of $\max 0.0707 \mathrm{~mL}(\mathrm{~V}=1 \mathrm{~cm} \times 3.1416 \mathrm{x}$ $\left.(0.15 \mathrm{~cm})^{2}=0.0707 \mathrm{~cm}^{3}=0.0707 \mathrm{ml}\right)$. The average platelet count in tubes of $1 \mathrm{~cm}$ length is 70.678.571 PLTs and in tubes of $28 \mathrm{~cm}$ length amounted to 1.979 .000 .000 PLTs. 


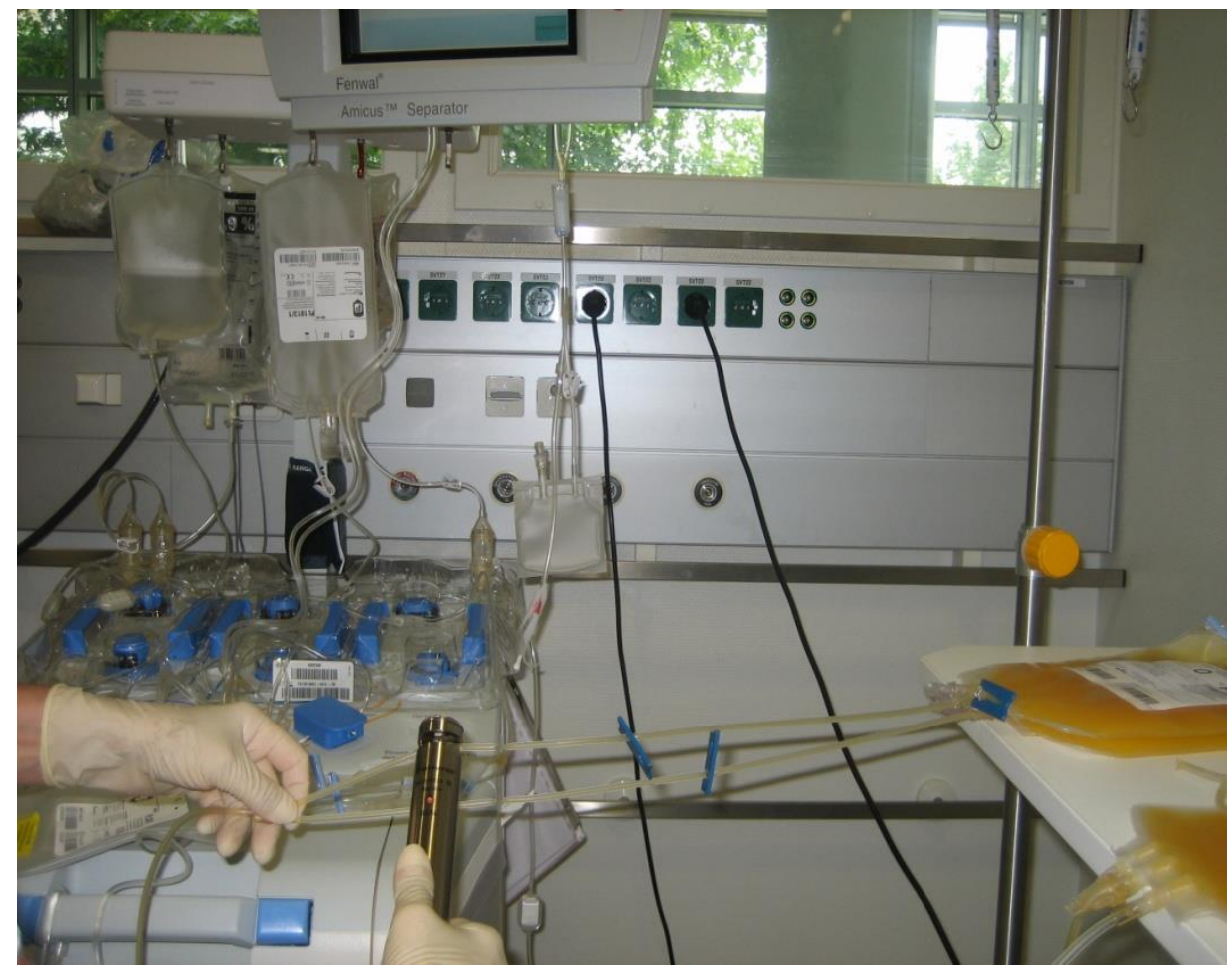

Figure IV-3: Disconnection of the tube of plateletpheresis set after donation

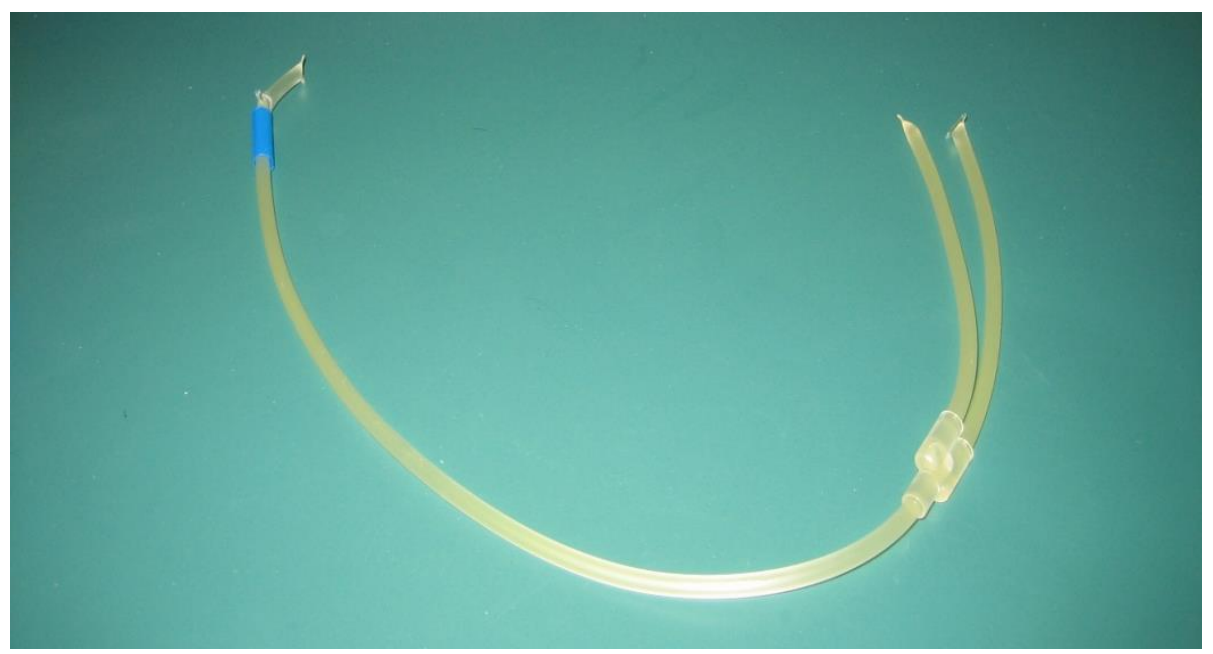

Figure IV-4: Flexible tube filled with plateletpheresis product after disconnection from the apheresis set from a double donation 
Table IV-1: Summary of count $(\mathrm{n}=)$ from plateletpheresis concentrate $(\mathrm{PC})$ samples and tube samples for analysis on day 0 and day 5 (red numbers belong to the irradiated samples)

\begin{tabular}{|c|c|c|c|c|c|c|c|c|c|c|c|c|c|c|c|c|c|c|c|}
\hline & \multicolumn{19}{|c|}{ Count of samples for specimen analysis $(n=)$} \\
\hline & & & & & & & & & & & \multicolumn{4}{|c|}{ Impedance aggregometry } & \multicolumn{5}{|c|}{ Light transmission aggregometry } \\
\hline & 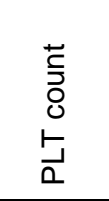 & $\stackrel{\underline{a}}{\underline{\underline{a}}}$ & I & 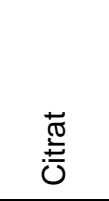 & 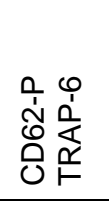 & 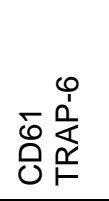 & 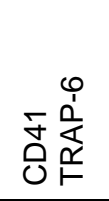 & 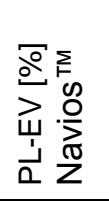 & 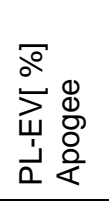 & 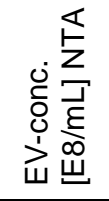 & 号 & 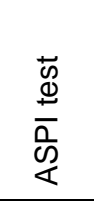 & 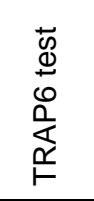 & 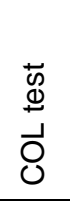 & 虽 & $\begin{array}{l}\frac{c}{\bar{d}} \\
\bar{\Xi} \\
\overline{\overline{0}} \\
0\end{array}$ & 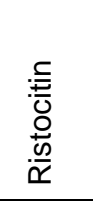 & 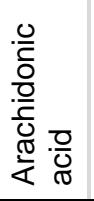 & $\begin{array}{l}\stackrel{0}{1} \\
\stackrel{1}{\mathbb{L}} \\
\mathbb{1} \\
\vDash\end{array}$ \\
\hline $\begin{array}{l}P C \\
\text { day } 0\end{array}$ & $27 / 15$ & $26 / 15$ & $27 / 15$ & $15 / 15$ & $27 / 15$ & $15 / 15$ & $15 / 15$ & $15 / 15$ & $15 / 15$ & $15 / 15$ & - & - & - & & - & - & & - & - \\
\hline $\begin{array}{l}P C \\
\text { day } 5\end{array}$ & $45 / 21$ & $10 / 15$ & $27 / 15$ & - & $44 / 18$ & $32 / 18$ & $32 / 18$ & $45 / 21$ & $0 / 15$ & $0 / 15$ & $19 / 11$ & $15 / 0$ & $25 / 6$ & $1 / 0$ & $12 / 6$ & $3 / 1$ & $12 / 1$ & $7 / 1$ & $3 / 16$ \\
\hline $\begin{array}{l}\text { Tube } \\
\text { day } 0\end{array}$ & $40 / 0$ & - & - & - & $39 / 0$ & $39 / 0$ & $38 / 0$ & $40 / 0$ & - & - & 29 & 2 & 36 & - & 24 & - & - & 2 & 27 \\
\hline $\begin{array}{l}\text { Tube } \\
\text { day } 5\end{array}$ & - & - & - & - & - & - & - & - & - & - & - & - & - & - & - & - & - & - & - \\
\hline $\begin{array}{l}\text { All } n \\
\text { day } 0\end{array}$ & 82 & 41 & 42 & 30 & 81 & 69 & 68 & 70 & 15 & 15 & 29 & 2 & 36 & - & 24 & - & - & 2 & 27 \\
\hline $\begin{array}{l}\text { All } n \\
\text { day } 5\end{array}$ & 66 & 25 & 42 & - & 62 & 50 & 50 & 66 & 15 & 15 & 30 & 15 & 31 & 1 & 35 & $0 / 1$ & $0 / 1$ & 8 & 19 \\
\hline
\end{tabular}




\subsection{Sample preparation of platelet concentrates for measurement of platelet-derived and red blood cell-derived extracellular vesicles}

\subsubsection{Dilution of PC samples}

Analysis of plasmatic and platelet-derived extracellular vesicles of PCs was performed on samples diluted with PBS-Dulbecco (DPBS), w/o Ca ${ }^{2+} / \mathrm{Mg}^{2+}$ Buffer (Biochrom AG, Berlin). For measurement of PL-EVs by flow cytometry with Navios ${ }^{\mathrm{TM}}$ (Beckman Coulter $\mathrm{GmbH}$, Krefeld ), PC samples was diluted first at 1:500 ratio and analyzed in a final dilution of 1:2500. PC samples for measurement with A50-Micro Flow Cytometer (Apogee Flow Systems Ltd., UK) were diluted first at 1:100 and used in a final dilution of 1:500.

Analysis of plasmatic EVs by nanoparticle tracking analysis (NTA) with NanoSight NS500 (NanoSight Ltd, Amesbury, UK) was performed on PC samples diluted at a ratio of 1:500.

\subsubsection{Platelet- and EV-containing plasma preparation}

Aggregometry was carried out in centrifuged PC samples at $336 \times \mathrm{g}$ for $10 \mathrm{~min}$ at room temperature to remove erythrocytes, leukocytes and most platelets and to obtain platelet rich plasma (PRP). Additionally, PRP was centrifuged anew at $1500 \times \mathrm{g}$ for $10 \mathrm{~min}$ at room temperature to produce platelet poor plasma (PPP).

\subsubsection{Preparative isolation of EVs from plateletpheresis concentrates}

EVs from PCs were isolated on day 0 and day 5 by differential centrifugation. Platelets were eliminated by centrifugation twice at $1.500 \times \mathrm{g}$ for $15 \mathrm{~min}$, followed by filtration of the supernatant through $1 \mu \mathrm{m}$ diameter pore filter. To pellet EVs, the filtrate was centrifuged at $30.000 \times \mathrm{g}$ for $45 \mathrm{~min}$ and the pellet was washed twice with $40 \mathrm{~mL}$ Tyrode-Hepes buffer and stored at $-80^{\circ} \mathrm{C}$ until analysis was carried out. The aforementioned protocol was used to isolate EVs from thrombocyte concentrate $(200 \mathrm{~mL})$ for preparative purposes (lipidomics, transcriptomics and proteomics) and is a refinement of the protocol published in PienimaekiRoemer et al. Transfusion July 2012.

\subsection{Sample preparation of red blood cell units for measurement of platelet-derived and red blood cell-derived extracellular vesicles}

EVs from red blood cell units were isolated by differential centrifugation. At first, one sample of RBC unit (25ml) was diluted with 25ml PBS-Dulbecco, w/o Ca ${ }^{2+} / \mathrm{Mg}^{2+}$ Buffer (Biochrom AG, Berlin) and subjected to centrifugation for 20 min twice at $1850 \mathrm{xg}$ in order to eliminate red blood cells. Subsequently, the obtained supernatant was centrifuged for $20 \mathrm{~min}$ at $3200 \mathrm{x} \mathrm{g}$. 
In addition, the supernatant was centrifuged by ultracentrifugation once at $120.000 \times \mathrm{g}$ for 90 min, followed by wash steps with PBS, three times at $120.000 \times \mathrm{g}$ for $90 \mathrm{~min}$. Next, the pellet (P) was resuspended in $2 \mathrm{ml}$ PBS, supplemented with an enzyme inhibitor ( 1 to $2 \mu \mathrm{L}$ ) and the mixture was stored at $5^{\circ} \mathrm{C}$ until the analysis was performed ( 2 to 3 days). All vesicles were divided in subfractions (fraction I, II, III) by density gradient centrifugation with OptiPrep Density Gradient Medium in diverse solutions (10\%, 15\%, 20\%, 25\%, and 30\%) at $40.000 \times \mathrm{g}$ for $3 \mathrm{~h}$. Subfractions were captured with a $27 \mathrm{G}$ needle, aliquoted and stored at $-80^{\circ} \mathrm{C}$.

Table IV-2: Preparation of red blood cell units for analysis of extracellular vesicles by ultracentrifugation and density gradient centrifugation

\begin{tabular}{|c|c|c|}
\hline Preparation step & Procedure & \\
\hline 1 & \multicolumn{2}{|l|}{ undiluted RBC unit } \\
\hline 2 & \multicolumn{2}{|l|}{ dilution with PBS 1:1 } \\
\hline 3 & \multicolumn{2}{|l|}{$20 \min , 1850 \times g$} \\
\hline 4 & \multicolumn{2}{|l|}{$20 \min , 1850 \times g$} \\
\hline 5 & \multicolumn{2}{|l|}{$20 \min , 3200 \times g$} \\
\hline 6 & \multicolumn{2}{|l|}{$90 \mathrm{~min}, 120.000 \times \mathrm{g}$} \\
\hline 7 & \multirow{2}{*}{$\begin{array}{l}\text { first wash step } 90 \min 120.000 \times g \\
\text { second wash step } 90 \min 120.000 \times g\end{array}$} & ( \\
\hline 8 & & (pentel < (rL) \\
\hline 9 & \multicolumn{2}{|l|}{ third wash step $90 \mathrm{~min} 120.000 \times \mathrm{g}$} \\
\hline 10 & \multicolumn{2}{|l|}{$\circlearrowleft$ pellet $(P 4)$} \\
\hline 11 & \multicolumn{2}{|l|}{ fraction I } \\
\hline 12 & \multicolumn{2}{|c|}{ density gradient centrifugation } \\
\hline 13 & \multicolumn{2}{|l|}{ fraction III } \\
\hline
\end{tabular}

\subsection{Flow cytometry}

\subsubsection{Principles and parameters}

Flow cytometry is a routine laboratory method for the analysis of single living cells of a size ranging from $400 \mathrm{~nm}$ to $40 \mu \mathrm{m}$. Conventional flow cytometers are also used for the analysis of single extracellular vesicles within the range of approximately $300 \mathrm{~nm}$ up to $1 \mu \mathrm{m}$. Because of the limitations in resolution of conventional flow cytometry and the small size of extracellular 
vesicles, numerous techniques were developed for quantitative and qualitative analysis of vesicle populations of smaller diameters then $70 \mathrm{~nm}$ (260). The instruments used for the experiments in this thesis were standard flow cytometers as Navios ${ }^{\mathrm{TM}}$ (Beckman Coulter $\mathrm{GmbH}$, Krefeld), Canto ${ }^{\text {TM }}$ II (Becton Dickinson, Heidelberg) and a high-resolution flow cytometer, the A50-Micro Flow Cytometer (Apogee Flow Systems Ltd, UK). All further descriptions and technical data refer to these instruments.

Prior to the analysis, cells were pushed from the sample tube through an injection tube. Then, sheath fluid pushed the cells through the flow chamber and positioned them in the middle of the laser beam by means of hydrodynamic focusing.

Two light scattering parameters are measured directly without further light filtering. Forward scatter (FSC) is a morphological parameter which is dependent on the relative size of the cell, whereas side scatter (SSC) is measured parallel to the laser beam and depends on the relative granularity or on homogeneity of the analyzed cell. Additional fluorescence channels can be used to investigate surface markers. For this purpose, optical filters are positioned in the light path to separate the signals emitted from the different fluorochromes. These fluorochromes absorb the laser light from the argon laser at $488 \mathrm{~nm}$ and emit at a specific longer wavelength. The signal is quantified with photomultipliers and further analyzed with the help of appropriate software.

\subsubsection{Platelet function test}

Externalization of platelet $p$-selectin (CD62P) in response to thrombin receptor activating peptide-6 (TRAP-6) as a measure of platelet activation marker was assessed on day 0 (day of production) and day 5 (after expiry date). The evaluation was carried out by flow cytometry according to standard protocols with evaluation of the expression of the major platelet membrane glycoproteins which support platelet adhesion and aggregation (GPIlb/GPIIla). The fluorescence of platelets was analyzed (FACSDiva Software, Version 6.1.3, Becton Dickinson, Heidelberg) and given in the mean fluorescent intensity (MFI) of positively stained platelets. This was related to the basal expression of $p$-selectin, granulophysin and the formed glycoproteins.

PC samples after resting storage on day 0 and agitated storage on day 5 were diluted with PBS-Dulbecco, w/o Ca ${ }^{2+} / \mathrm{Mg}^{2+}$ Buffer (Biochrom AG, Berlin) to a final concentration of platelets of $\leq 20000 / \mu \mathrm{L}$. Diluted PC samples $(20 \mu \mathrm{L})$ were incubated with $10 \mu \mathrm{L}$ TRAP-6 in molar mass of $10 \mu \mathrm{M}$ for 10 minutes (min) versus control with $10 \mu \mathrm{L}$ PBS-Dulbecco, w/o Ca $\mathrm{Ca}^{2+} / \mathrm{Mg}^{2+}$ Buffer at room temperature in darkness. Immunostaining was performed with the monoclonal antibodies CD62P-FITC, CD41-PE and CD61-PerCP according to the standard protocols of manufacturer. 
Table IV-3: Scheme of immunostaining for analyzing PLT function test

$20 \mu \mathrm{L}$ of diluted PC sample was incubated with fluorescence labeled antibodies after activation of platelets in vitro by thrombin receptor activating peptide-6 (TRAP-6, Bachem Biochemica, Heidelberg). Standard labeling was performed with three platelet specific antibodies. All antibodies were used for elaborated analysis of platelet function

\begin{tabular}{|c|c|c|}
\hline CD41-PE & CD61-PerCP & CD62P-FITC \\
\hline $20 \mu \mathrm{L}$ & $20 \mu \mathrm{L}$ & $20 \mu \mathrm{L}$ \\
\hline
\end{tabular}

Flow cytometry measurements were performed with the standard 3-colour instrument setting (FACS Canto ${ }^{\mathrm{TM}}$ II, BD). For each sample, data were collected until the 10,000-event stop criterion was met. The whole platelet population was gated on forward and side scatter with adjusted gains. Gating strategy was applied to forward scatter (FSC) and to side scatter (SSC) according to CD41 and/ or CD61 positively stained platelet population; subsequently, percentage of CD62P positive platelets was gated.

.

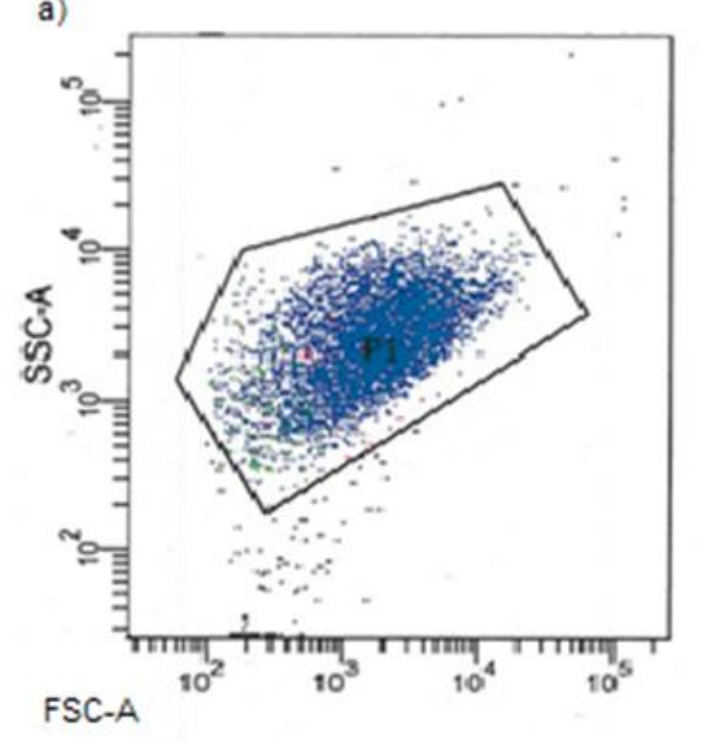

b)

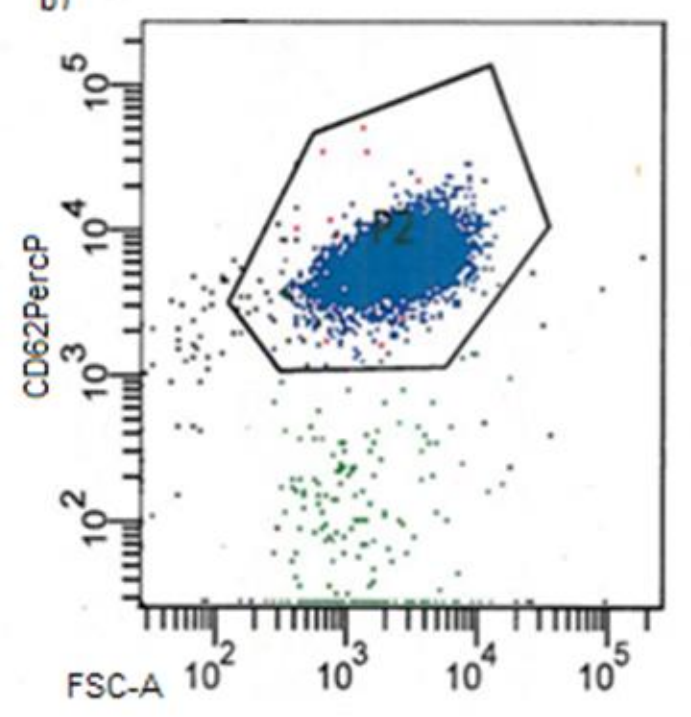

Figure IV-5: Gating strategy by flow cytometry with Canto ${ }^{\mathrm{TM}}$ ॥I

a) Gating of CD61 (not shown) labeled platelets on FSC to SSC (P1). b) Gate of P1 corresponds to platelet populations labeled with CD62P to SSC (P2). Events of lower intensity are the equivalent of platelet debris and were not considered as a correct population. 


\begin{tabular}{|c|c|c|c|c|}
\hline a) & \multicolumn{2}{|c|}{ CD62P FIT... } & \multicolumn{2}{|c|}{ CD41 PE-A CD61 Per... } \\
\hline Population & \#Events & Mean & Mean & Mean \\
\hline P2 & 4,910 & 6.079 & 16,547 & 11,429 \\
\hline P3 & 4,890 & 6,088 & 16,614 & 11,445 \\
\hline b) & \multirow{2}{*}{\multicolumn{2}{|c|}{ CD62P FIT... }} & & \\
\hline Population & & Meân & $\begin{array}{r}\text { CD41 PE-A } \\
\text { Meàn }\end{array}$ & $\begin{array}{r}\text { CD61 Per... } \\
\text { Mean }\end{array}$ \\
\hline P2 & 4,811 & 363 & 7,699 & 6,500 \\
\hline P3 & 4,791 & 341 & 7,730 & 6,481 \\
\hline
\end{tabular}

Figure IV-6: Absolute values of platelet events and Mean Fluorescence Intensity by analytical FACSDiva Software

The CD62P Mean Fluorescence Intensity (MFI, without dimension) of a) activated platelets (incubated with TRAP-6) was calculated in percent to basal CD62P MFI of b) platelets without activation (incubated with PBS-Dulbecco) in vitro set up to 100\%.

a)

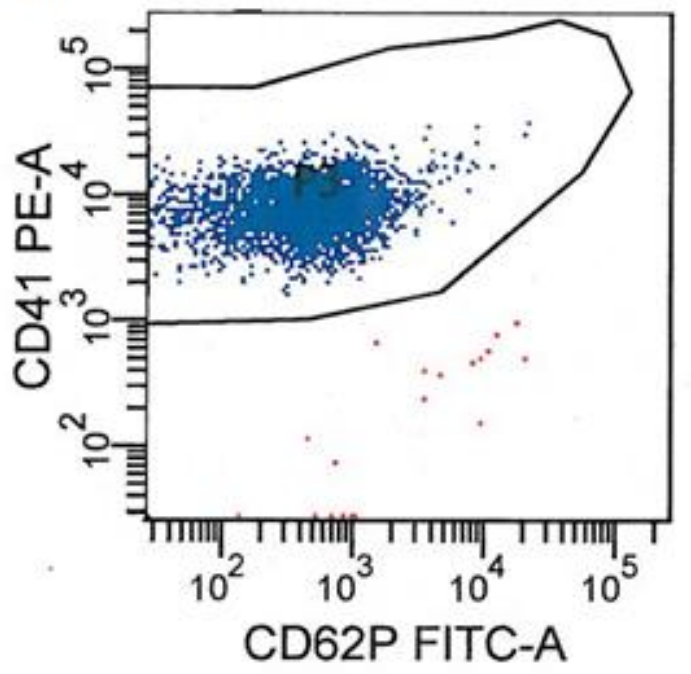

b)

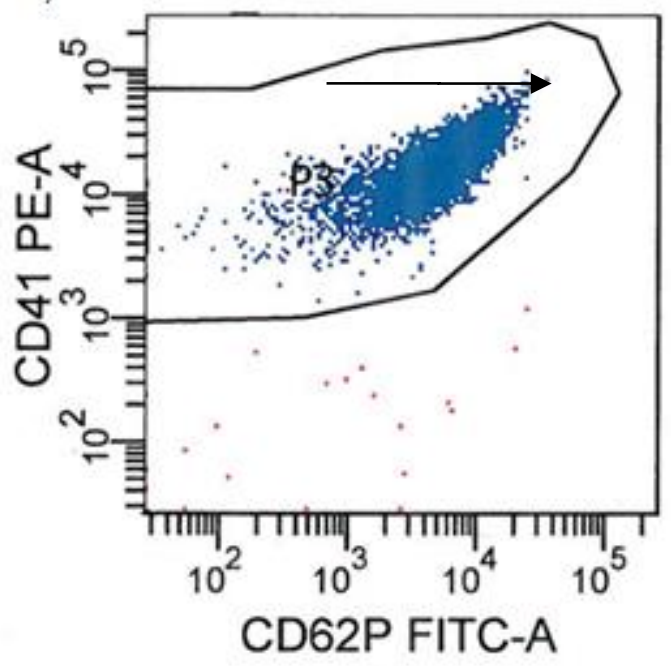

Figure IV-7: Changes of externalization of CD62P after activation with TRAP-6 by flow cytometry

a) Gated platelets (P3) with basal activatability (MFI $=100 \%$ ) and b) activated after stimulation with TRAP-6 $(\mathrm{MFI}=\mathrm{x} \%)$. Platelet population shifts to the right of the $x$-axis $(\rightarrow)$ which indicates higher intensity of CD62P externalization. As expected, PC stimulated with CD62P with sufficient quality characteristics amounts at least to $200 \%$ on day 0 and at least to $140-150 \%$ on day 5 .

\subsubsection{Platelet-derived extracellular vesicles}

\subsubsection{Navios $^{\mathrm{TM}}$}

The flow cytometer (Navios ${ }^{\mathrm{TM}}$, Beckman Coulter, Krefeld, Germany) scatter size was calibrated by fluorescent polystyrene beads with a refractive index (RI) of 1.61 (Megamix beads; BioCytex, 
Marseille, France) to discriminate platelets (approx. $0.9-3.0 \mu \mathrm{m}$ ) from platelet-derived extracellular vesicles (PL-EVs; approx. $0.5-0.9 \mu \mathrm{m}$ ) as described earlier (261). Staining of 100 $\mu \mathrm{L}$ of 1:500 diluted platelet rich plasma (PRP in phosphate buffered saline, PBS-Dulbecco w/o $\left.\mathrm{Ca}^{2+} / \mathrm{Mg}^{2+}\right)$ was performed by incubation with saturating amount $(2.5 \mu \mathrm{L})$ of $\mathrm{R}$ PhycoerythrinCyanine (PC)7-labeled mouse IgG 1 against CD61 glycoprotein GPIIla (IOT®, Beckman Coulter) at room temperature for $10 \mathrm{~min}$ in darkness. After addition of $500 \mu \mathrm{L}$ PBS (final dilution of $1: 2500)$, samples were analysed immediately as described. Each sample run produced data of 500,000 events occurring within 180 seconds in a defined volume of samples. The relative PL$\mathrm{EV}$ and platelet counts were used to calculate the count of released PL-EVs/ platelet (as ratio in $\%$ ). IgG1-PC7 (IOT®, Beckman Coulter) was used as isotypic control to detect unspecific binding.
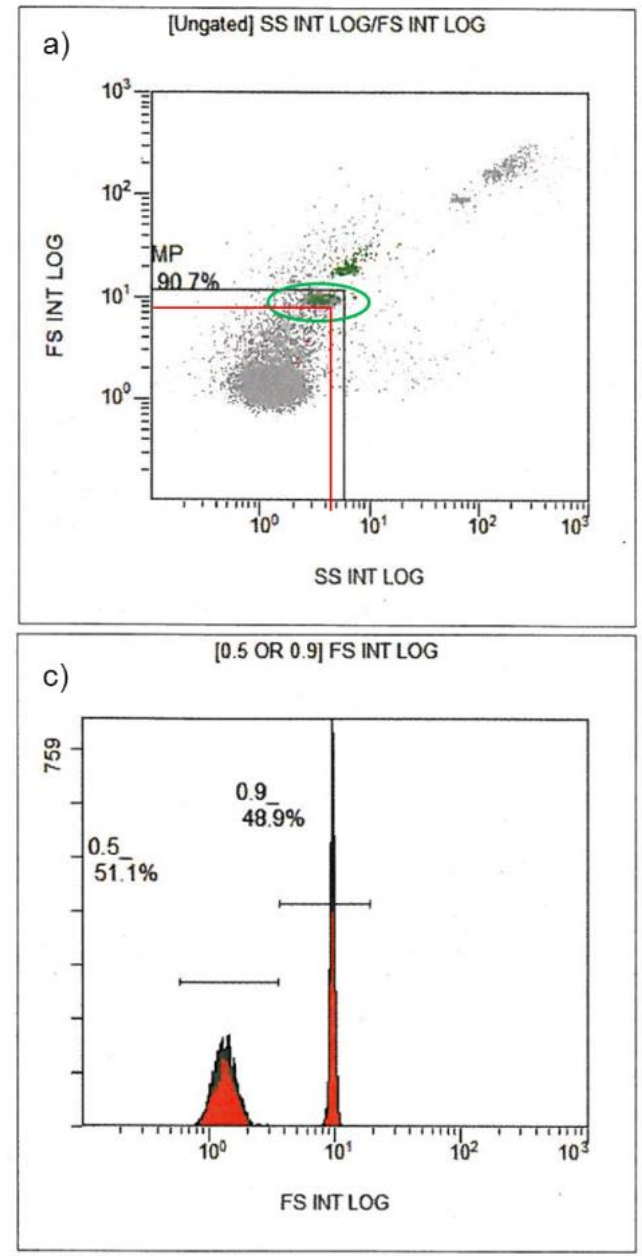

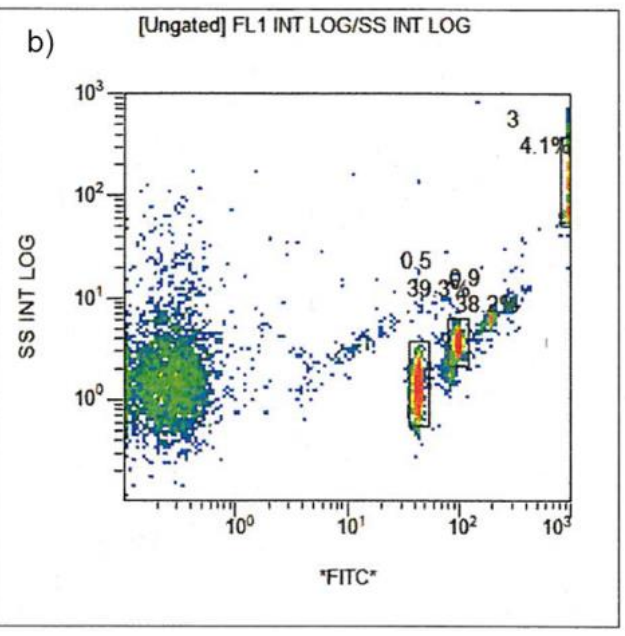

Gates in FITC/SS INT LOG:

$A=$ Beads mit $0,5 \mu \mathrm{m}$

$B=$ Beads mit $0,9 \mu \mathrm{m}$

$\mathrm{C}=$ Beads mit 3,0 $\mu \mathrm{m}$

Figure IV-8: Calibration of instrument settings for EV-analysis with Navios ${ }^{\mathrm{TM}}$

a) EV gate (pictured as red box) excludes $0.5 \mu \mathrm{m}$ bead population (green circled). b) Gates in FITC/SS INT LOG belongs to bead populations: $0.5 \mu \mathrm{m}, 0.9 \mu \mathrm{m}, 3.0 \mu \mathrm{m}$, the green bulk corresponds to the instrument noise. c) FS INT LOG for $0.5 \mu \mathrm{m}$ bead rate is measured up to $50 \%$. 


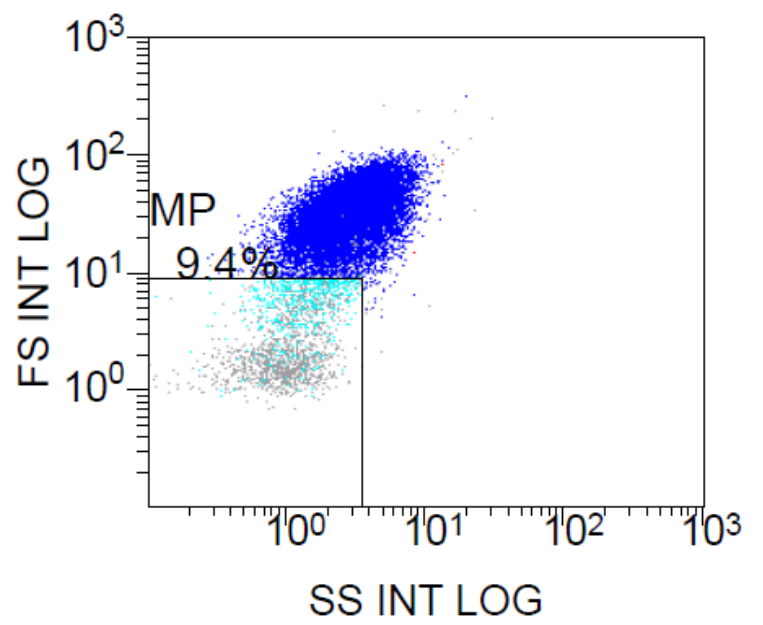

Figure IV-9: Gating strategy for EV analysis by flow cytometry with Navios ${ }^{\mathrm{TM}}$

Gating of PL-EVs (light blue) smaller than $500 \mathrm{~nm}$ corresponds to the gating strategies of bead gating.
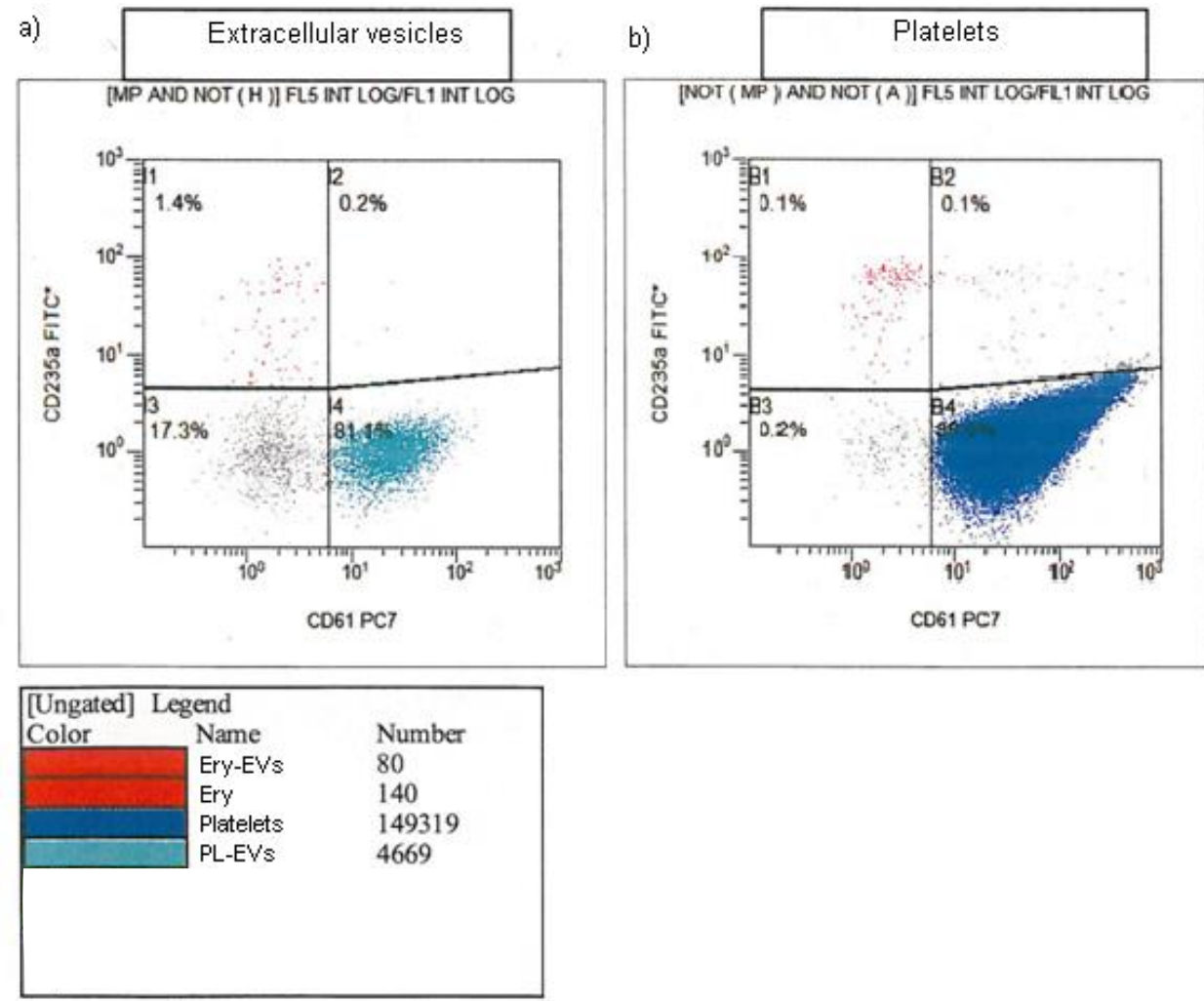

Figure IV-10: Scatter plots of events for platelets and platelet-derived EVs by flow cytometry with Navios ${ }^{\mathrm{TM}}$

a) CD61-PC7 positive labeled PL-EVs according to the size calibration with $500 \mathrm{~nm}$ beads. b) Platelets labeled with CD61-PC7 distinguishable from PL-EVs. The box below presents the legend with data of the occurred events. The PL-EV and platelet counts were used to calculate the count of released PL-EVs per platelets (in \%). For representative results, semi quantitative determination of PL-EVs on day 0 was $3.12 \%$. PL-EVs of PC amount to $5-10 \%$ on day 0 and at least to $10 \%$ on day 5 . 


\subsubsection{FACS Canto ${ }^{\mathrm{TM}}$ II}

The flow cytometer FACS Canto ${ }^{\mathrm{TM}}$ II (Becton Dickinson, Heidelberg) scatter size was calibrated by a bead mix (Apogee Flow Systems Ltd, UK) with eight subpopulations of beads which differ in size and refractive index (RI). The bead mix contains silica spheres of the following diameters: $180 \mathrm{~nm}, 240 \mathrm{~nm}, 300 \mathrm{~nm}, 590 \mathrm{~nm}, 880 \mathrm{~nm}$ and $1300 \mathrm{~nm}$ with a refractive index of $\eta=1.42$, and $110 \mathrm{~nm}$ and $500 \mathrm{~nm}$ green fluorescent beads with a refractive index of $\eta=1.59$ (latex). Staining of $100 \mu \mathrm{L}$ 1:500-diluted platelet rich plasma (PRP in phosphate buffered saline, PBS-Dulbecco w/o Ca ${ }^{2+} / \mathrm{Mg}^{2+}$ ) was performed by incubation with saturating amount $(2.5 \mu \mathrm{L})$ of R Phycoerythrin-Cyanine (PC)7-labeled mouse IgG 1 against CD61 glycoprotein GPIIla (IOT®, Beckman Coulter) at room temperature for 10 min in darkness. After addition of $500 \mu \mathrm{L}$ PBS, samples were immediately analysed. For each sample run, data were acquired for up to 15,000 events of the longest time of $3 \mathrm{~min}$, in a defined volume of sample. The relative PL-EV and platelet counts were used to calculate the count of released PL-EVs/ platelets (in \%).

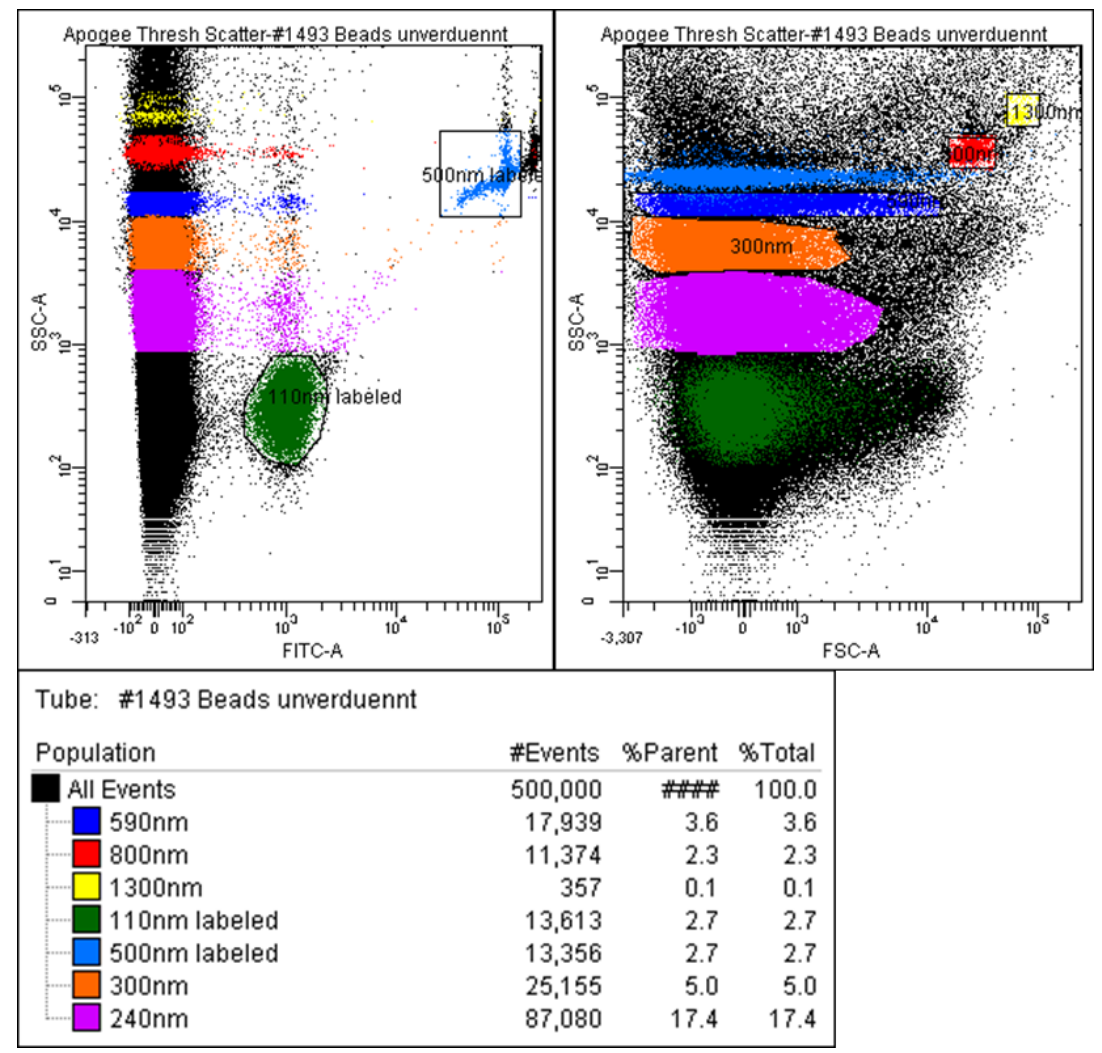

Figure IV-11: Calibration of the instrument settings for EV-analysis with Canto ${ }^{\mathrm{TM}}$ II

Fluorescent bead populations $(110 \mathrm{~nm}, 500 \mathrm{~nm}$ ) and non-flourescent bead populations (240 $\mathrm{nm}, 300 \mathrm{~nm}, 590 \mathrm{~nm}, 800 \mathrm{~nm}, 1300 \mathrm{~nm}$ ) of the bead mix are presented. 180nm beads are not distinguishable from $240 \mathrm{~nm}$ beads and are included in this bead population. 


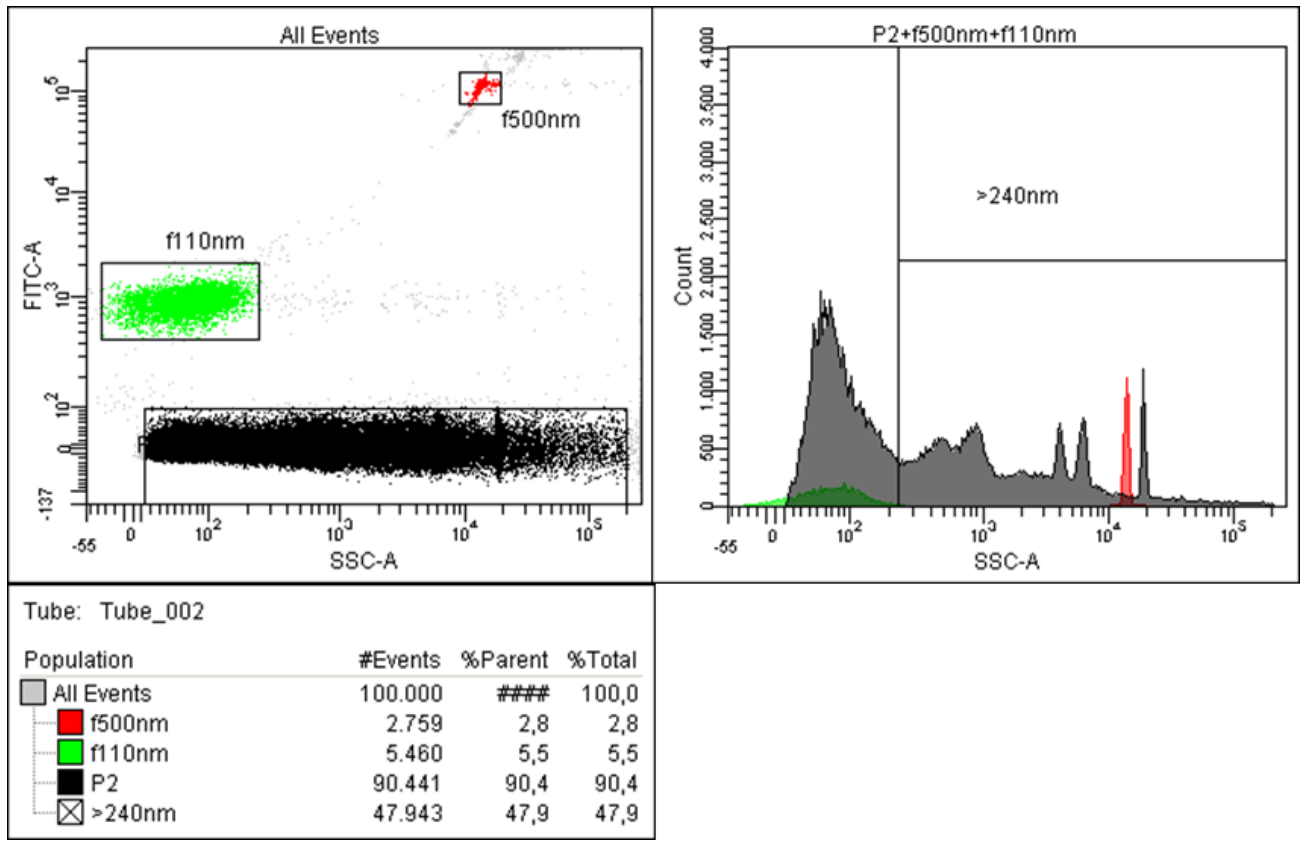

Figure IV-12: Gating strategies for EV-analysis by flow cytometry with Canto ${ }^{\mathrm{TM}}$ II

Two fluorescent bead populations of the bead mix $(110 \mathrm{~nm}, 500 \mathrm{~nm})$ are presented in the upper right histogram. Instrument noise overlapped with the $110 \mathrm{~nm}$ bead population and was excluded from the analysis of events $240 \mathrm{~nm}$.

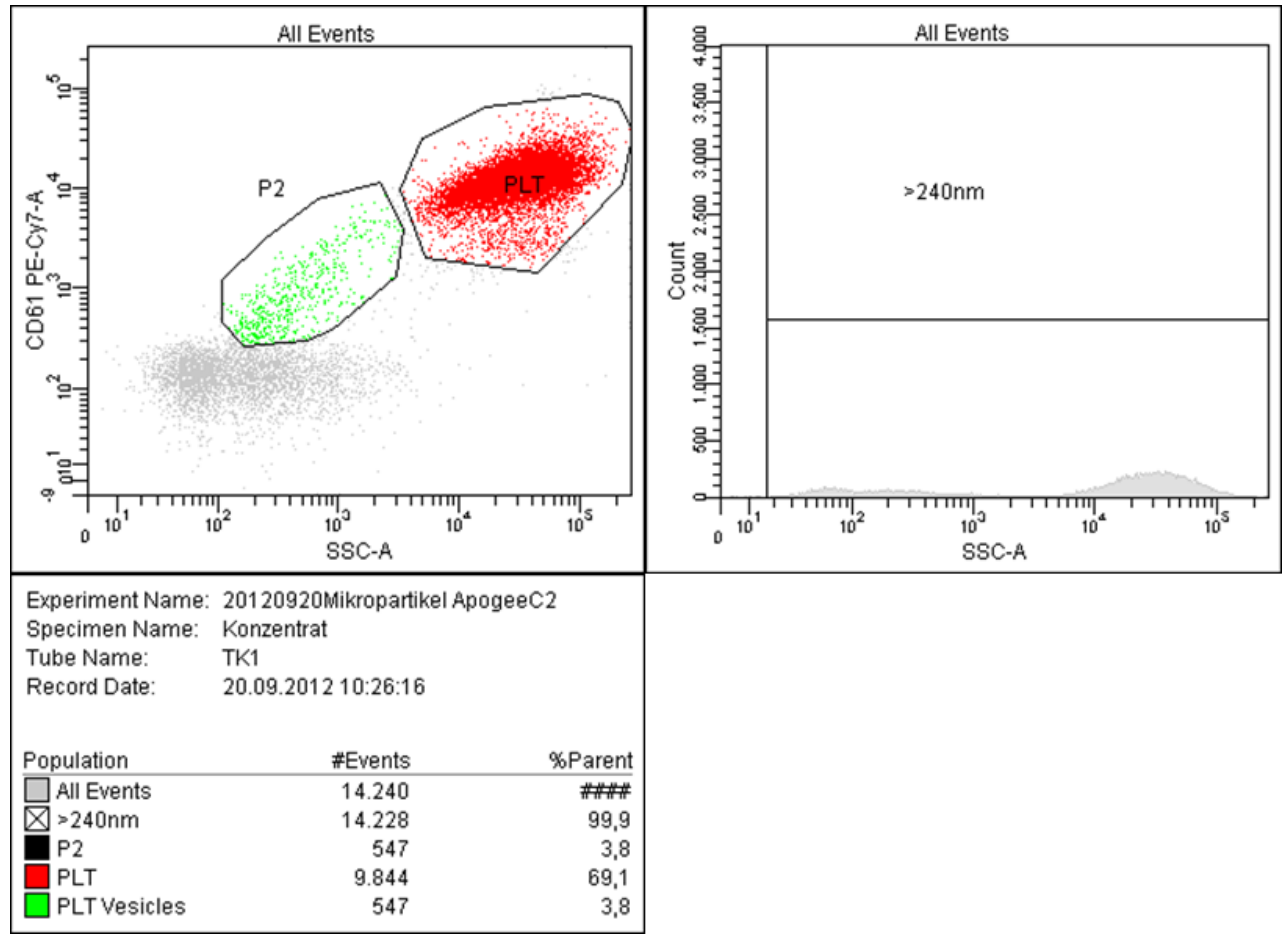

Figure IV-13: Platelet-derived EV analysis by flow cytometry with Canto ${ }^{\mathrm{TM}}$ ॥

The same $>240 \mathrm{~nm}$ gate of the calibration setting with beads was applied for the measurement of EVs from PC samples. Top left: discrimination of platelet population and PL-EVs in SSC. 


\subsubsection{Apogee}

\subsection{Background}

In contrast to a conventional flow cytometer, the Apogee A50-Micro model offers a resolution of a much higher light scatter sensitivity and absolute count ability for EV subpopulations without the use of count beads. According to manufacturer's information, the Small Angle Light Scatter (SALS: identical to Forward Scatter or FSC) sensitivity is higher than that of $110 \mathrm{~nm}$ silica beads (NanoSight, $100 \mathrm{~nm}$ beads) and the Large Angle Light Scatter (LALS: identical to Side Scatter or SSC) sensitivity is higher than that of $110 \mathrm{~nm}$ silica beads.

\subsection{Practical details}

The flow cytometer Apogee A-50 Micro (Apogee Flow Systems Ltd, UK), fitted with 2 light scatters (LS), 4 fluorescent filter blocks (FL) and a blue laser (488 nm, $50 \mathrm{~mW}$ ), was used to analyse PL-EVs from PC samples on day 0 and day 5 . The scatter size was calibrated by a bead mix (Apogee Flow Systems Ltd, UK) containing silica spheres with the following diameters: $180 \mathrm{~nm}, 240 \mathrm{~nm}, 300 \mathrm{~nm}, 590 \mathrm{~nm}, 880 \mathrm{~nm}$ and $1300 \mathrm{~nm}$ diameter with a refractive index of $\eta=1.42$, and $110 \mathrm{~nm}$ and $500 \mathrm{~nm}$ green fluorescent beads with a refractive index of $\eta=1.59$ (latex). The beads were used to set up the EV gate in FSC and SSC with a voltage of $250 \mathrm{~V}$ for FSC and a voltage of $270 \mathrm{~V}$ for SSC. The threshold was set at 8 (or 65535) units for FSC and 8 (or 65535) units for SSC to discriminate the smallest beads (110 nm) from instrument noise (determinate with PBS).

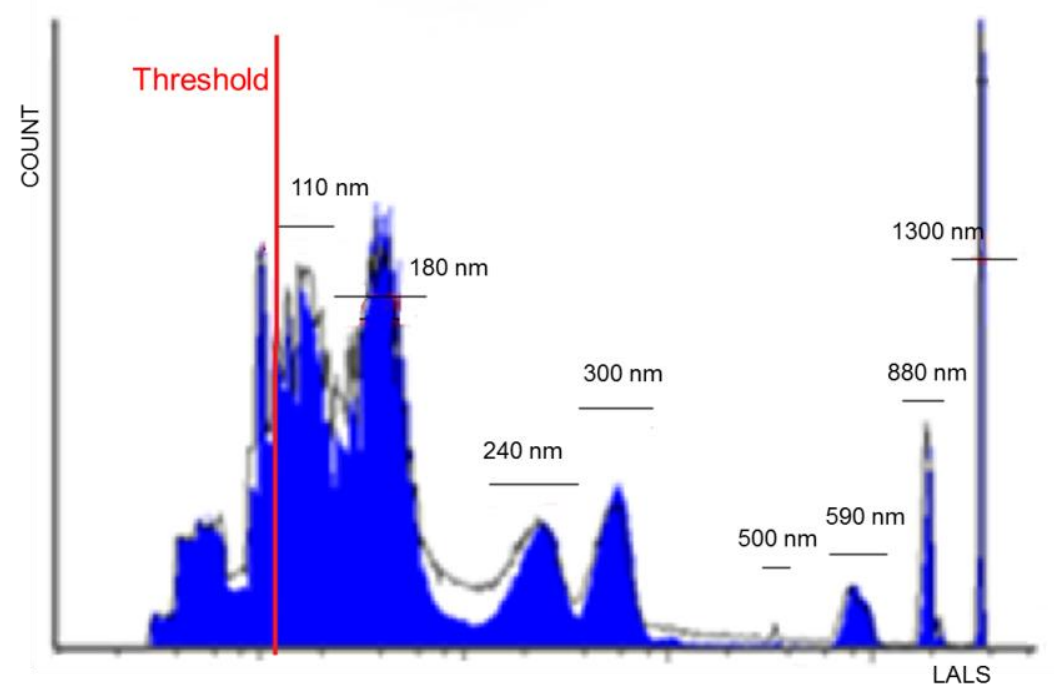

Figure IV-14: Histogram of calibration of the instrument settings of Apogee A-50 Micro

The bead mix (Apogee) includes bead populations with diameters $110 \mathrm{~nm}, 180 \mathrm{~nm}, 240 \mathrm{~nm}$, $300 \mathrm{~nm}, 500 \mathrm{~nm}, 590 \mathrm{~nm}, 880 \mathrm{~nm}$ and $1300 \mathrm{~nm}$ and are presented as blue peaks under the gates (black horizontal lines). 
Staining of $100 \mu \mathrm{L}$ 1:100-diluted PRP (in phosphate buffered saline, PBS) was performed by incubation with saturating amount of $R$ Phycoerythrin-Cyanine (PC)7-labeled mouse $\lg _{1}$ against CD61 glycoprotein GPIIla (IOTest $\AA /$ Beckman Coulter) at room temperature for $10 \mathrm{~min}$ in darkness. After addition of $500 \mu \mathrm{L}$ PBS, samples were analysed immediately for CD61positive events in the far red zone by gating for platelets $(2-3 \mu \mathrm{m})$ and PL-EVs in the range of values lower than $180 \mathrm{~nm}$ (size: S), 180 - $300 \mathrm{~nm}$ (size: M) and 300 - $590 \mathrm{~nm}$ (size: L). Flow rate was standardized to $1.39 \mu \mathrm{L} / \mathrm{min}$ and a sample run of $130 \mu \mathrm{L}$ to maintain the number of events/ sec amounting to approximately 2,000-5,000. Samples was acquired within at least 90 seconds. The concentration of PLT/ $\mu \mathrm{L}, E V s / \mu \mathrm{L}$ was calculated from the flow rate and the measurement time. Data were compared by analysing events/ $\mu \mathrm{L}$. IgG1-PC7 (IOT®, Beckman Coulter) was used as isotypic control to detect unspecific binding.

\subsection{Nanoparticle Tracking Analysis}

\subsubsection{Background}

Nanoparticle tracking analysis (NTA) is based on light scattering, which illuminates particles in suspension. Light is induced by a laser beam, therefore, rapidly moving particles are visualized by an optical microscope equipped with a conventional video camera (CCD: Charge-coupled Device) to capture them and produce a movie. Vesicles are present as small spheres of light (262). Software program dynamically analyses the paths the particles take under Brownian motion over a defined period of time captured by the camera. Speed of motion or diffusion coefficient $(D t)$ is related to the size of each particle. The software selects trajectories whose lifetime is sufficiently long to ensure statistically accurate results and it measures the average distance, which the particle covers per frame. The NTA software calculates the particle size with the particle movement velocity by tracking individual particles and their positional changes in two dimensions. This calculation is based on the Stokes-Einstein equation:

$$
D t=\frac{K_{B} T}{6 \pi \eta r_{h}}
$$

In the equation $D t$ is the diffusion coefficient (the mean squared displacement in two dimensions, $\left.<x, y>^{2}\right), K_{B}$ stands for the Boltzmann's constant, $T$ is the temperature of solvent in Kelvin, $\eta$ means solvent viscosity and $r_{h}$ corresponds to the hydrodynamic radius. The measured Dt of a particle by NTA belongs to a spherical particle, as assessed by StrokesEinstein equation, and is further ascribed to the correlating diameter of the particle.

The estimated concentration of particles is based on the assumed scattering volume calculated from the dimensions of the vision's field. The sample chamber is approximately $500 \mathrm{~nm}$ deep and the depth of laser beam is around $20 \mu \mathrm{m}$ at the time of analysis. The average concentration per scattering volume is estimated by counting the number of tracked particles. 


\subsubsection{Practical details}

For NTA the NanoSight NS500 system (NanoSight, Amesbury, UK) was used, equipped with a green laser (Excitation 532 nm, Emission 565 nm long pass) and an EMCCD-Camera (EMCCD high sensitivity camera) with $20 \times$ objective magnification applied as a standard. Calibration of instrument settings was carried out with 1:500 diluted (PBS-Dulbecco w/o Ca ${ }^{2+} / \mathrm{Mg}^{2+}$ Buffer, Biochrom AG, Berlin) monodispers solutions of polystyrene beads in diameter of $50 \mathrm{~nm}, 100$ $\mathrm{nm}, 200 \mathrm{~nm}$ and $400 \mathrm{~nm}$ (Nanosight, Amesbury, UK).

All measurements of native PC samples without additives (diluted 1:500 with DPBS) were performed under the same conditions as following: temperature of $22^{\circ} \mathrm{C}$, camera level at 9 , high threshold at $9882 \pm 50$ and tracking period time of $120 \mathrm{sec}$, during which the videos corresponding to the settings for high polydispersity and high reproducibility of the values are made. The PC samples were diluted in a total volume of $1000 \mathrm{~mL}$ to achieve the recommended particle concentration ranging between $10^{8} / \mathrm{mL}$ and $10^{9} / \mathrm{mL}$ for optimum determination of particle concentration, shown as 40 particles per image (PPI) in the movie. The pre-processing settings were used in the auto-capture mode.

Staining of PL-EVs was not implemented; hence the analyzed particle population includes not only PL-EVs, but also immunocomplexes and lipid vesicles.

\section{8 $\quad$ Aggregometry}

\subsubsection{Impedance aggregometry}

Platelet aggregability of PCs on day 5 was analysed by impedance aggregometry on the Multiplate $\AA$ platelet function analyser (Multiplate Analyzer, Software Version 2.02.11, Dynabyte $\mathrm{GmbH}$, Munich, Germany) using various platelet agonists to induce in vitro activation and platelet-to-platelet adhesion. Immediately after sample collection, platelet rich plasma (PRP) was centrifuged at $1500 \mathrm{~g}$ for $10 \mathrm{~min}$ at room temperature to generate platelet poor plasma (PPP). PRP was diluted in PPP to a concentration of 400 platelets/ $\mathrm{nL}$.

For analysis using Multiplate $\AA, 300 \mu \mathrm{L}$ of the plasma sample was diluted in a ratio of $1: 1$ with $\mathrm{NaCl}$ (for ASPItest) or with $\mathrm{NaCL}-\mathrm{CaCl}_{2}$ (for COLtest, ADPtest and TRAPtest) and stimulated with agonists yielding the following final concentrations at $37^{\circ} \mathrm{C}$ : ADP $(6.5 \mu \mathrm{mol} / \mathrm{L})$, collagen $(3.2 \mu \mathrm{g} / \mathrm{mL}$ ), arachidonic acid (AA, $0.5 \mathrm{mmol} / \mathrm{L})$, Thrombin-Receptor-Activating-Peptide (TRAP)-6 (32 $\mu \mathrm{mol} / \mathrm{L})$. Multiplate assay measures changes in electrical resistance given as aggregation units over time ( $\left.\mathrm{AU}^{*} \mathrm{~min}\right)$.

\subsubsection{Light transmission aggregometry}

Platelet aggregation analysis of PCs after 5 days of storage was carried out by Born aggregometry on the APACT 4 S Plus (Rolf Greiner BioChemica GmbH, Flacht Germany) using 
various platelet agonists to induce in vitro activation and platelet-to-platelet adhesion. Immediately after sample collection, platelet rich plasma (PRP) was centrifuged at $1500 \mathrm{~g}$ for 10 min at room temperature to generate platelet poor plasma (PPP). PRP was diluted in PPP to a concentration of 400 platelets/ $\mathrm{nL}$. For Born aggregometry, $200 \mu \mathrm{L}$ diluted plasma sample were stimulated by agonists yielding the following final concentrations at $37^{\circ} \mathrm{C}$ : ADP $(20 \mu \mathrm{mol})$, collagen $(10 \mu \mathrm{g} / \mathrm{mL})$, ristocetin $(1.5 \mathrm{mg} / \mathrm{mL})$, arachidonic acid $(A A, 0.5 \mathrm{mg} / \mathrm{mL})$, thrombinreceptor-activating-peptide (TRAP)-6 $(0.1 \mathrm{mM})$. Maximal aggregation of platelets was registered as a change of transmission of light within at least $8 \mathrm{~min}$ as percentage of aggregation $(0-100 \%)$ and was compared to that for PPP alone.

\subsection{Total blood count and platelet count analysis}

Blood counts of donors were measured on the XE-5000 hematology analyzer (Sysmex, Norderstedt) with impedance measurement by using hydrodynamic focusing technologies and flow cytometry according to the standard procedures using ethylenediamine tetra-acetic acid (EDTA) blood samples on day 0. Platelet count and immature platelet factor (IPF) of donor and PC samples were measured by the fluorescent optical method and impedance measurement was conducted by hydrodynamic focusing technologies and flow cytometry. Analysis of PCs is based on the internally defined standard procedures for blood concentrates (validated method) with diluted PC samples $(50 \mu \mathrm{L})$ in capillary tubes (Sysmex, Germany) with $200 \mu \mathrm{L}$ Cellpackbuffer (Sysmex, Germany) in a mixed ratio of 1:5.

\subsection{Photometry}

Serum parameters of glucose and lipid metabolism were determined by bichromatic and polychromatic endpoint measurement on the clinical chemistry analyzer Dimension Vista $₫ 1500$ (Siemens Healthcare Diagnostics) according to standard procedures of manufacturer. Tests included the measurement of cholesterol $(540 \mathrm{~nm}, 452 \mathrm{~nm}, 700 \mathrm{~nm}$ ), LDL-cholesterol (540 nm, $700 \mathrm{~nm})$, HDL-cholesterol (600 nm, $700 \mathrm{~nm})$, triglyceride $(510 \mathrm{~nm}, 700 \mathrm{~nm})$ and calcium blood as well as non-fasting serum glucose $(340 \mathrm{~nm}, 383 \mathrm{~nm})$ of donor blood samples with clot activator (day 0).

\subsection{Nephelometry}

Serum parameters of lipid metabolism and the initial point of complement cascade in donor blood samples with clot activator (day 0) including apolipoprotein A-I, apolipoprotein B100 and complement $\mathrm{C} 3$ and $\mathrm{C}$ reactive protein (CRP) were determined by nephelometry on the clinical 
chemistry analyzer Dimension Vista® 1500 (Siemens Healthcare Diagnostics) according to standard procedures.

In addition, lipoprotein (a) and apolipoprotein E were determined by nephelometry on the clinical chemistry analyzer BN ProSpec (Siemens Healthcare Diagnostics) according to standard procedures.

\subsection{Enzym-Linked Immunosorbent Assay (ELISA)}

Serum parameters of lipid metabolism in donor blood samples with clot activator (day 0), i.e. apolipoprotein B48, apolipoprotein C-I, and clusterin (apo J), were determined by a Sunrise ${ }^{\mathrm{TM}}$ Tecan Photometer at $450 \mathrm{~nm}$. Tests conformed to the standard procedures of a quantitative sandwich enzyme immunoassay technique with polyclonal and biotinylated polyclonal antibodies specific for human apolipoproteins which were recognized by a streptavidinperoxidase conjugate. The intensity of color was measured.

\subsection{Chemiluminescence immunoassay (CLIA)}

Serum insulin-like growth factor 1 (IGF1) and insulin-like growth factor-binding protein 3 (IGFPB3) were determined in blood samples with clot activator from donors on day 0 using an automated CLIA analyzer Immulite 2000 XPi (Siemens Healthcare Diagnostics) according to manufacturer's protocols.

\subsection{Coagulometry}

Multifibren $U$ reagent (Siemens Healthcare Diagnostics) is a bovine thrombin reagent and was used in the modified Clauss determination of fibrinogen in citrate blood samples from donors on day 0 by coagulometry in the BCS $\AA$ System (Siemens Healthcare Diagnostics) according to standard protocols of manufacturer.

\subsection{Chromogenic method}

The Berichrom Antithrombin III (A) Kit (Siemens Healthcare Diagnostics) is a chromogenic activity assay and was used in the BCS® System (Siemens Healthcare Diagnostics) to measure antithrombin III (ATIII) in citrate blood samples from donors on day 0 before apheresis. The Berichrom PAI Kit (Siemens Healthcare Diagnostics) is a chromogenic test system and was used in the BCS $\circledast$ System (Siemens Healthcare Diagnostics) to analyze plasminogen activator inhibitor -1 (PAI-1) in blood samples according to the standard protocols of manufacturer. 


\subsection{Immunturbidometry}

$\mathrm{HbA1c}$ measurements were performed in ethylenediamine tetra-acetic acid (EDTA) blood samples from donors on day 0 with the help of the Hemoglobin A1c Kit (Roche Diagnostics, Germany) by immunturbidimetric method on the Cobas Integra (Roche Diagnostics, Germany) according to the standard protocol of manufacturer.

\subsection{Potentiometric method}

The measurement of $\mathrm{pH}$-values was performed in PC samples (Blood Gas Monovette®, Sarstedt, Germany) on day 0 and day 5 at $22^{\circ} \mathrm{C} \pm 2^{\circ} \mathrm{C}$ by potentiometric method on ABL 90 FLEX (Radiometer $\mathrm{GmbH}$, Willich) according to the standard protocols of manufacturer and to the intern validated method for $\mathrm{pH}$-analysis of platelet concentrates.

\subsection{Statistical analysis}

Data were collected and organized in Microsoft Excel spreadsheets. Summary files containing means of all experimental data were assembled to facilitate graphic and statistical evaluation of the data. For statistical computing, the corresponding data were imported and analyzed using the statistics package SPSS 20 (IBM). In all experiments, statistically significant differences or correlation were considered when $p$ values were less than $<0.05\left(^{*}\right),<0.01\left(^{* *}\right)$ and $<0.001{ }^{\left({ }^{* *}\right)}$. Graphical presentations were carried out by using SigmaPlot 8.0 and SPSS 19.0 softwares. Graphs were created in Excel 2010 (Microsoft), SPSS 20 (IBM) or in Sigmaplot 8.0 (Systat Software). The applied tests and the resulting $p$-values are mentioned in the figure legends. 


\section{Results}

\subsection{Results of methodological issues}

\subsubsection{Validation of PL-EV measurement with standard flow cytometry}

First, a validation of the semiquatitative analysis of PL-EVs in PCs was performed. The aim was to define the sensitivity of the method (see Figure $\mathrm{V}-1, \mathrm{~A}$ ). The saturating amount of the monoclonal antibody (moAb) CD61 (PE-Cy7) was titrated in three PC samples on day 0. Maximal saturation of $1 \mu \mathrm{L}$ moAb solution was required to saturate CD61 epitopes in 100,000 platelets absolute count according to the manufacturer's protocols. Absolute platelet count in PC samples was about 197,400 platelets per assay. Saturation of moAb was detectable already at the level of $0.5 \mu \mathrm{L}$ of moAb, for both PLTs and PL-EVs.

In order to define accuracy of the method (see Figure V-1, B), a single, three-day stored PC was analyzed in triplicate. Platelet poor plasma (PPP) of this PC was aliquoted and subjected to analysis. The same standard configurations were applied to all samples.

A minor deviation of the PL-EV values and a recovery rate of $97.85 \%$ to $99.44 \%$ in samples with PPP could be assigned, indicative that the accuracy of method is acceptable.

Proof of linearity was limited, possibly due to the fact that the presented method cannot be applied to the absolute quantification of PL-EVs. Three PC samples on day 3 were analyzed as controls and compared to the same samples, but spiked with autologous PPP (see Figure V-1, C). As a result, a linear increase of PL-EVs was observed. However, statistical analysis of the data is not achievable since bead-based calibrators with the same refractive index and density as PL-EVs are currently not available. Therefore, the amount of PL-EVs [in \%] in PC samples without spiking was outlined as $100 \%$ and was related to PL-EVs [in \%] of spiked samples $(+1$ $\mu \mathrm{L}$ PPP, $+2 \mu \mathrm{L}$ PPP, $+3 \mu \mathrm{L}$ PPP, $+4 \mu \mathrm{L}$ PPP). Data of PL-EVs from PCs show a linear coherence between increased volume of spikes and amount of PL-EVs (dashed line in Figure $\mathrm{V}-1, \mathrm{C}$ depicts PL-EVs from PPP). Increase of PL-EVs reached $81.1 \%$ (mean) per addition of 1 $\mu \mathrm{L}$ PPP $(\mathrm{SD}=2.3)$.

In order to obtain reliable results of intra-assay variation (see Figure V-1, D), 5 samples of 3 PCs on day 2 (PC1, PC2, PC3) were analyzed under the same standard immunolabeling and flow cytometer settings, as described in 4.6.3.1. First, a measurement was performed without mixing of sample. The following measurements were performed with PC samples which were gently manually mixed. Coefficients of variation were determined for all PCs and amounted to $3.1 \%, 3.1 \%$ and $2.3 \%$, respectively. Analysis of stability of the method (see Figure V-1, E) was performed with 3 PC samples on day 2 (PC1, PC2, PC3) after 0-30 minutes, 1h, 2h, 4h and 24h after plateletpheresis. All samples were diluted in PBS-Dulbecco medium at a ratio of 1:500 and incubated with moAb CD61-PerCP at room temperature for 10 minutes. Each sample of a total volume of $500 \mu \mathrm{L}$ was measured within 180 seconds by flow cytometry (Navios ${ }^{\mathrm{TM}}$, Beckmann 
Coulter). The analyzer was previously calibrated with a standard calibration bead mix (Megamix Beads $(0.5 \mu \mathrm{m}, 0.9 \mu \mathrm{m}, 3.0 \mu \mathrm{m}$ Polystyrene-Beads, $\mathrm{Rl}=1.6)$. Coefficients of variation of the method stability within 24 hours were determined for PC1, PC2 PC3 at $3.0 \%, 4.9 \%$ and $7.3 \%$, respectively.
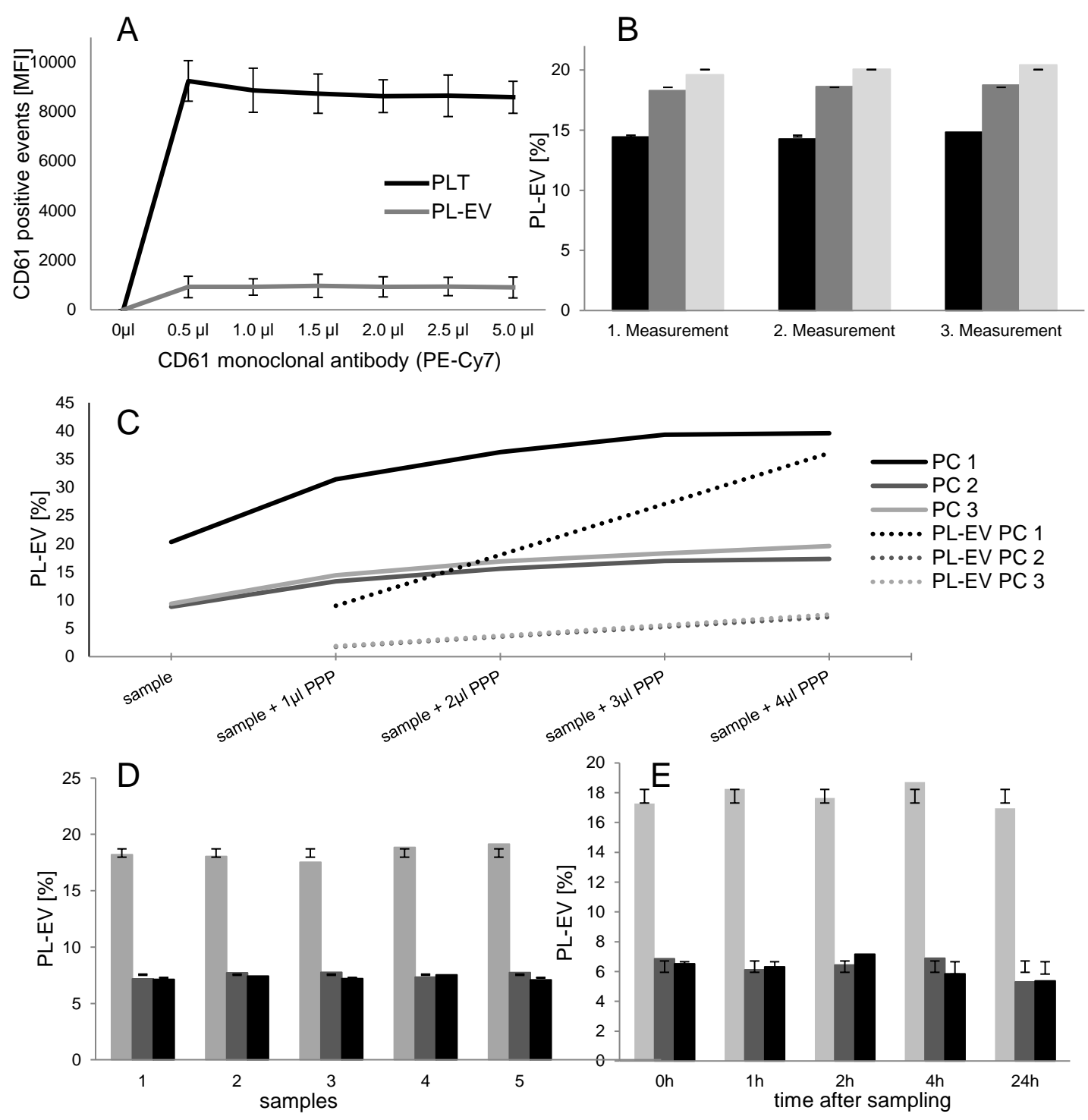

Figure V-1: Validation of PL-EV measurement by standard flow cytometry

A: Titration curve of saturating amount of moAb against CD61 as a sensitivity assay (PECy7=PC7). For the detection of the CD61 positive events of platelets and platelet-derived extracellular vesicles (PL-EV) [Mean fluorescent intensity $=\mathrm{MFI}$, without dimension] varying volumes of moAb CD61-PECy7 were added. B: Accuracy of the method for the analysis of PLEVs $(n=3)$ was proven by measurements in triplicate. C: Linearity assay of PL-EV analysis. Increase of PL-EV in PCs (continuous line) after addition of platelet poor plasma (PPP) of different volumes $(1 \mu \mathrm{L}, 2 \mu \mathrm{L}, 3 \mu \mathrm{L}, 4 \mu \mathrm{L})$. PL-EV amount in PPP (dashed lines). D: Intra assay precision examination of the method for PL-EV analysis $(n=3)$. E: 24h-stability of the measurement of PL-EVs $(n=3)$. Values of $A, B, D, E$ are presented as mean $\pm S D$. 


\subsubsection{Validation of nanoparticle tracking analysis}

In order to prove linearity of quantification of vesicles in a solution, an analysis of diluted samples was performed by nanoparticle tracking analysis (NTA, NS500; NanoSight, UK) with monodispers $100 \mathrm{~nm}$ diameter bead solution and different dilutions of PC samples. The solution of polystyrene beads was diluted with PBS-Dulbecco medium at a ratio of 1:20 to 1:10000 and measured with the same instrument settings. As pictured in Figure V-2, the curve depicting a dilution factor below 1:1000 runs parallel to the curve of the computed values of the bead concentrations per $\mathrm{mL}$. To confirm the data, the analysis was repeated with other sizes of monodispers bead solutions $(50 \mathrm{~nm}, 200 \mathrm{~nm})$ and similar results of linearity were obtained. When a bead solution is intensively mixed, NTA discriminates different bead populations according to their size and to their bead concentration in different dilutions. Subsequently, the obtained results were compared to the computed values. This procedure was successfully repeated in human vesicle preparations from plateletpheresis concentrates in order to test performance of linearity of concentrations and precision with triplet measurements in highly polydisperse samples.

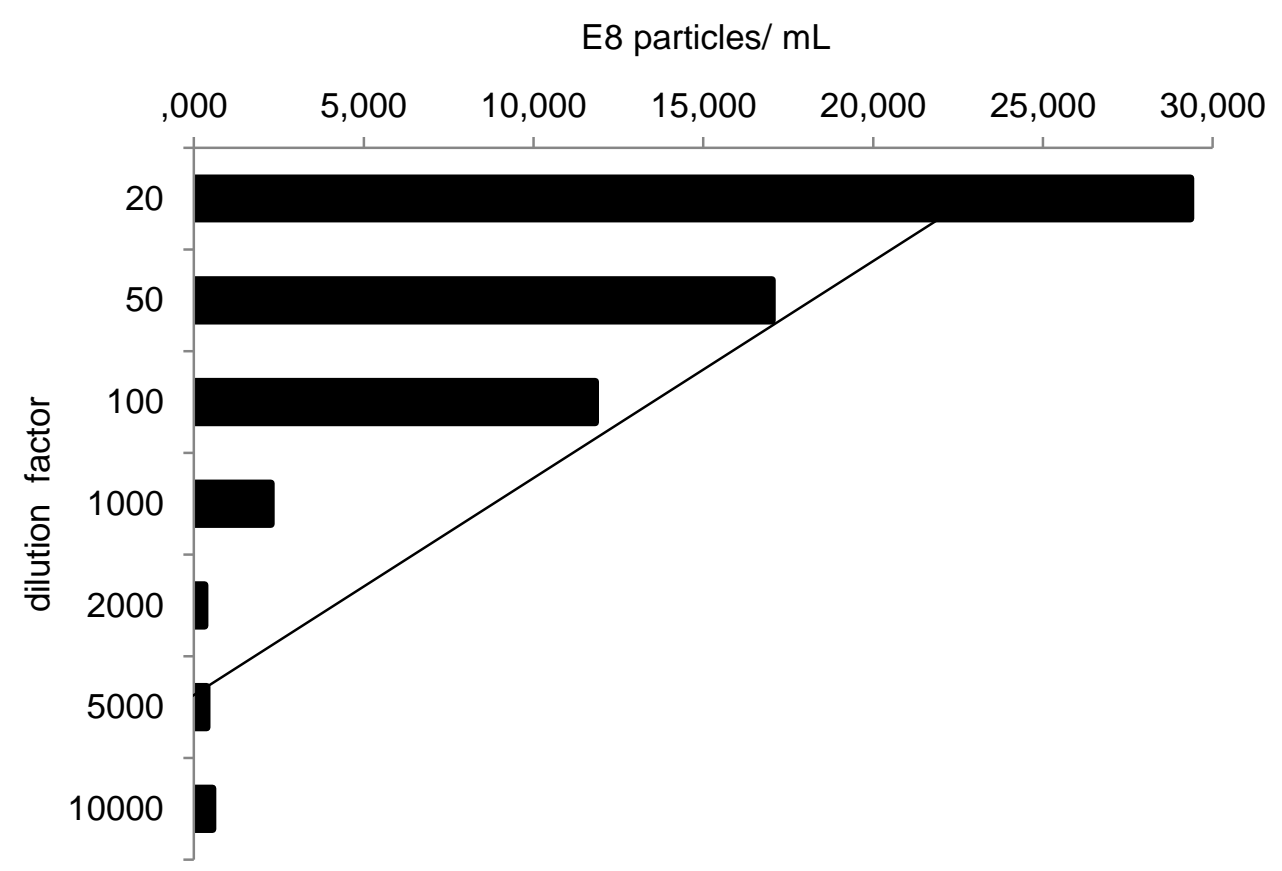

Figure V-2: Linearity of the method for nanoparticle tracking analysis (NTA) with beads

Concentrations of several diluted samples (1 to 20 down to 1 to 10000) of monodispers polystyrene bead spheres of size of $100 \mathrm{~nm}$ are shown as black bars and approximate linearity was proven for dilutions up to 1:1000. 

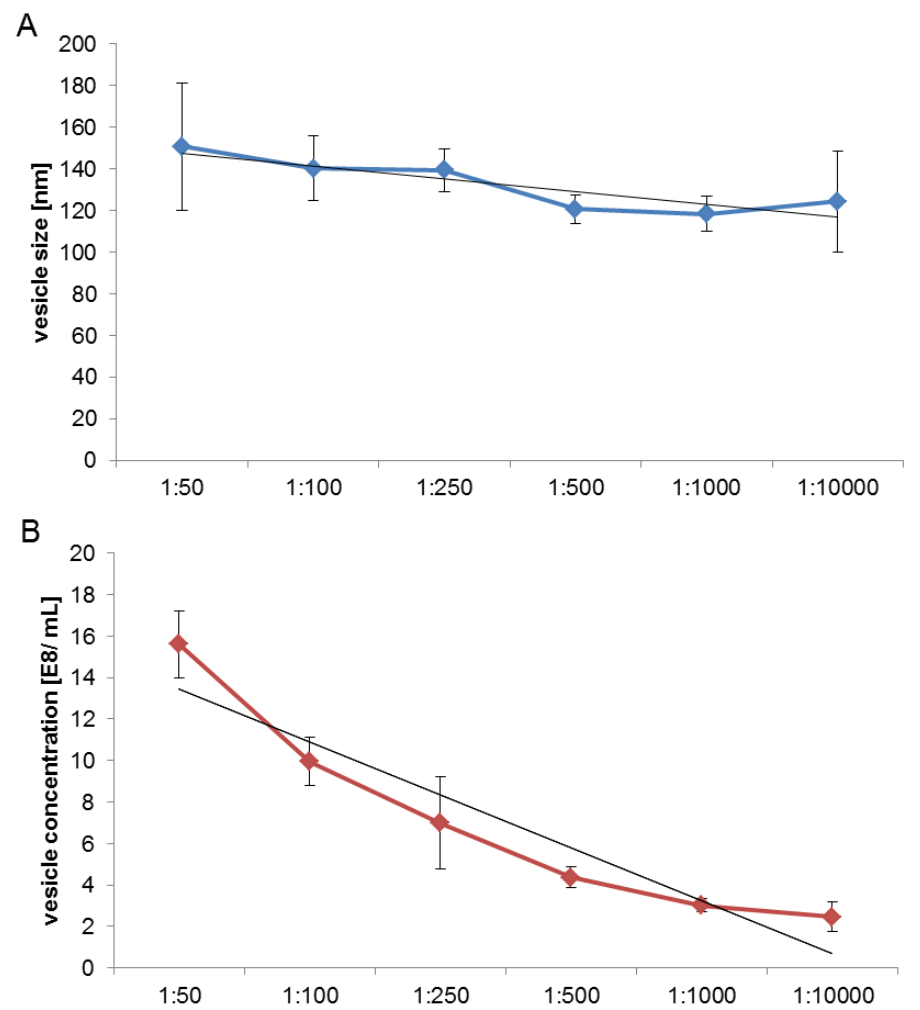

Figure V-3: Linearity and accuracy of plasma vesicle size analysis (A) and recovery and accuracy of plasma vesicle concentration (B) by NTA

Measurement in triplicate was performed with one fresh PCs of different dilutions with PBSDulbecco. In order to confirm accuracy of the method, coefficients of variation of size analysis were computed for the following dilution factors: 50, 100, 250, 500, 1000 and $10000(20.3 \%$, $11 \%, 7.3 \%, 5.6 \%, 7 \%$ and $19.4 \%)$ corresponding to particle concentration $(10.3 \%, 11.6 \%$, $31.7 \%, 11.8 \%, 9.8 \%, 28.3 \%)$.

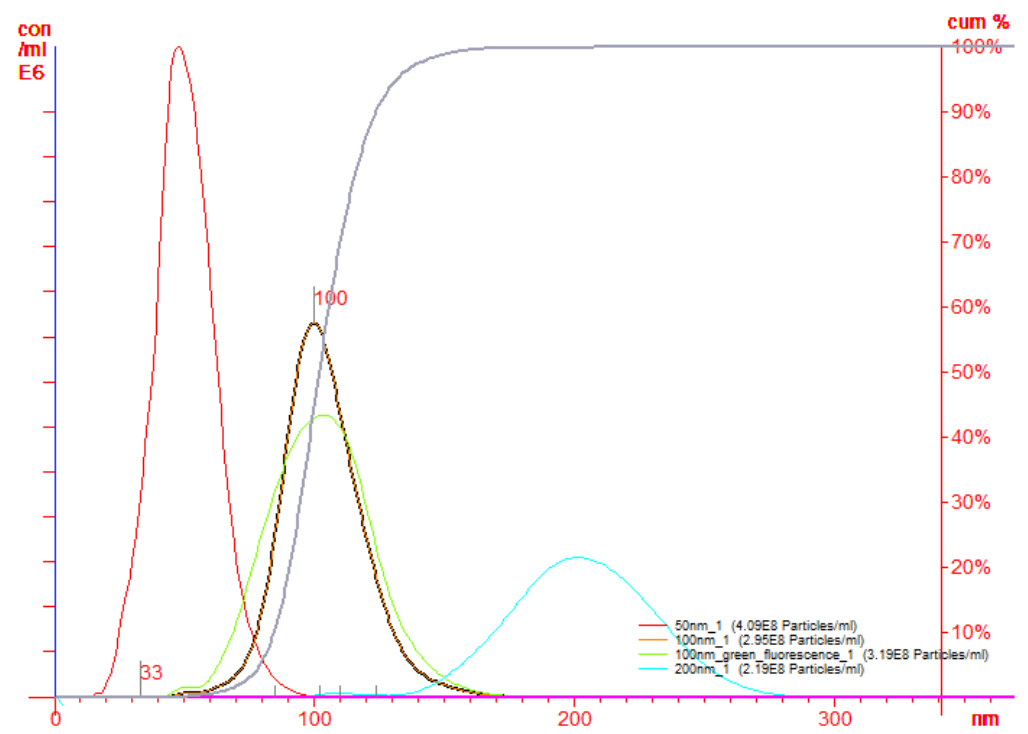

Figure V-4: Cumulative graph represents proof of size recovery for NTA with monodispers bead solutions

The red curve shows $50 \mathrm{~nm}$ sized beads, whereas the brown curve corresponds to $100 \mathrm{~nm}$ sized beads, the green one to the fluorescent beads $(100 \mathrm{~nm})$ and the blue curve depicts the $200 \mathrm{~nm}$ sized beads. 


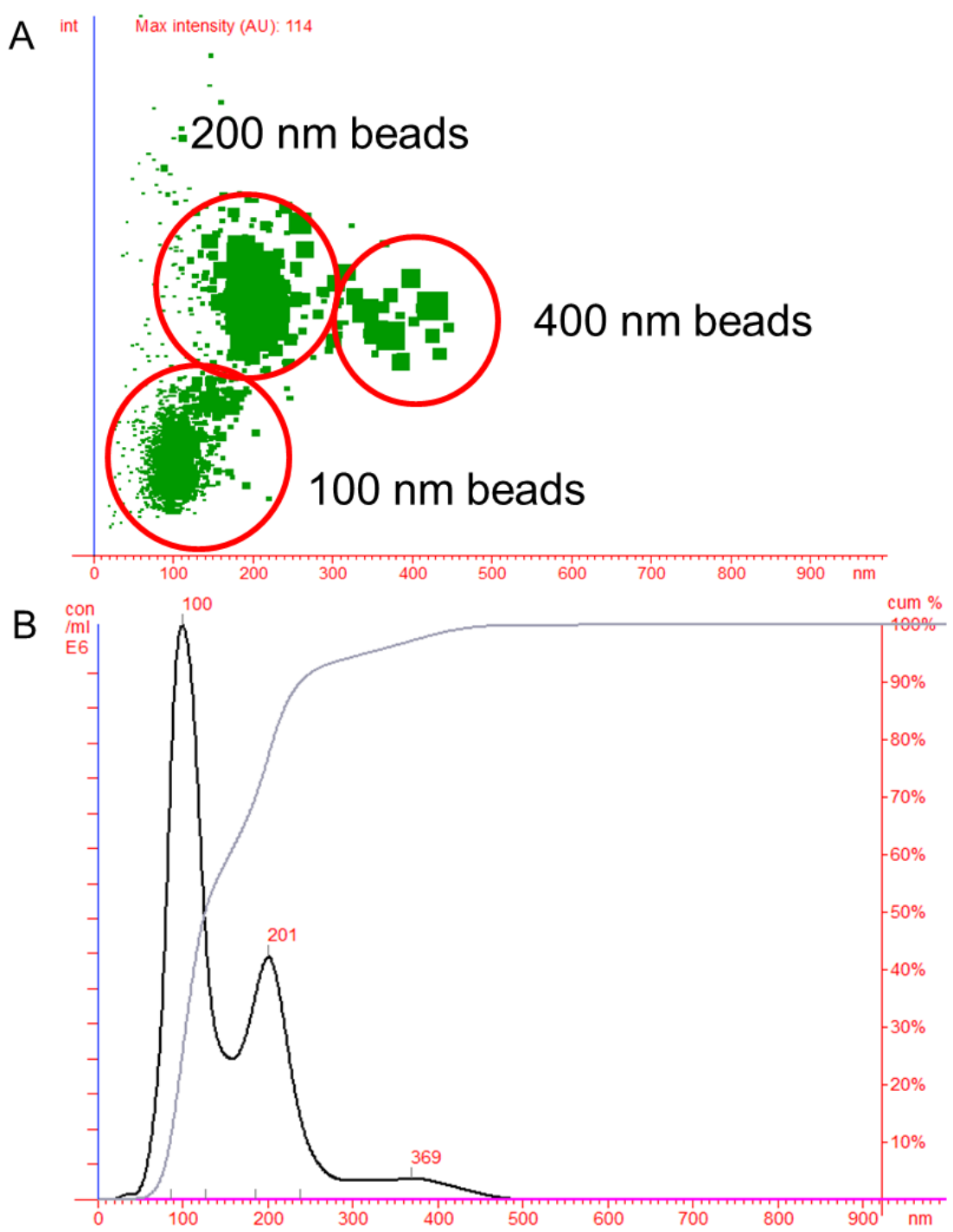

Figure V-5: Accuracy of size measurements with mixed up polystyrene bead solutions

Solutions of beads of size of $100 \mathrm{~nm}, 200 \mathrm{~nm}$ and $400 \mathrm{~nm}$ were mixed and diluted with PBS to achieve 1:100 dilution for analysis by NTA. The intensity of scattered light of each bead and its corresponding size is figured in panel $\mathrm{A}$. Red circles surround population of the same size. The diagram in panel $B$ represents concentration $\times 10^{6} / \mathrm{mL}$ versus the size of bead populations in $\mathrm{nm}$.

\subsubsection{Analysis of plasma-vesicle-measurement by different flow cytometers and nanoparticle tracking system}

In order to analyze EV four different analytical systems, two standard flow cytometers, one high sensitivity flow cytometer and one nanoparticle tracking system (NTA), were tested as tools to validate inter- and intra-assay performance. Both methods, flow cytometry and NTA, are not directly comparable with each other. The three flow cytometric methods remained in focus of the quantitative comparison of the results, while NTA system provides information related to 
changes of size distribution of blood products over storage time. Nonetheless, size distribution of all plasma vesicles, lipoproteins or plasma proteins and their complexes need to be discriminated by the NTA system to identify changes of blood products over storage time. With the purpose of inter-assay validation tests the same PC samples were examined by all instruments in parallel. Comparable results of size and concentration of plasma vesicles in PCs were obtained (see Table V-1 and Figure V-6) and NTA can be assumed as a reliable method of vesicle quantification and vesicle size distribution. Plasma vesicles including PL-EVs were identified between 40 and $530 \mathrm{~nm}$ with a main peak at approximately $100 \mathrm{~nm}$.

Table V-1: Vesicle quantification of plateletpheresis concentrates (PCs) by NTA

Immunolabeling was not required, when analyzing vesicle count and size. Dilution factor of the correct analysis of NTA software depends on the cumulative amount of vesicles in concentrates ( $n=6$, on day 4 and 7 ). Particle size values are reported as the absolute value of the most abundant subpopulation of vesicles.

\begin{tabular}{l|lll}
\hline & Particles/ ml & Range of size [nm] & Peak of size [nm] \\
\hline PC 1 (1:500) & $4.03 \times 10^{8}$ & $40-380$ & $100>172>340$ \\
PC 2 (1:1000) & $4.77 \times 10^{8}$ & $40-420$ & $77>141>217$ \\
PC 3 (1:500) & $3.69 \times 10^{8}$ & $40-380$ & $97>322$ \\
PC 4 (1:500) & $3.78 \times 10^{8}$ & $40-480$ & $110>206$ \\
PC 5 (1:500) & $2.25 \times 10^{8}$ & $50-530$ & $130>195$ \\
PC 6 (1:500) & $2.27 \times 10^{8}$ & $40-480$ & $170>260>370$ \\
\hline
\end{tabular}




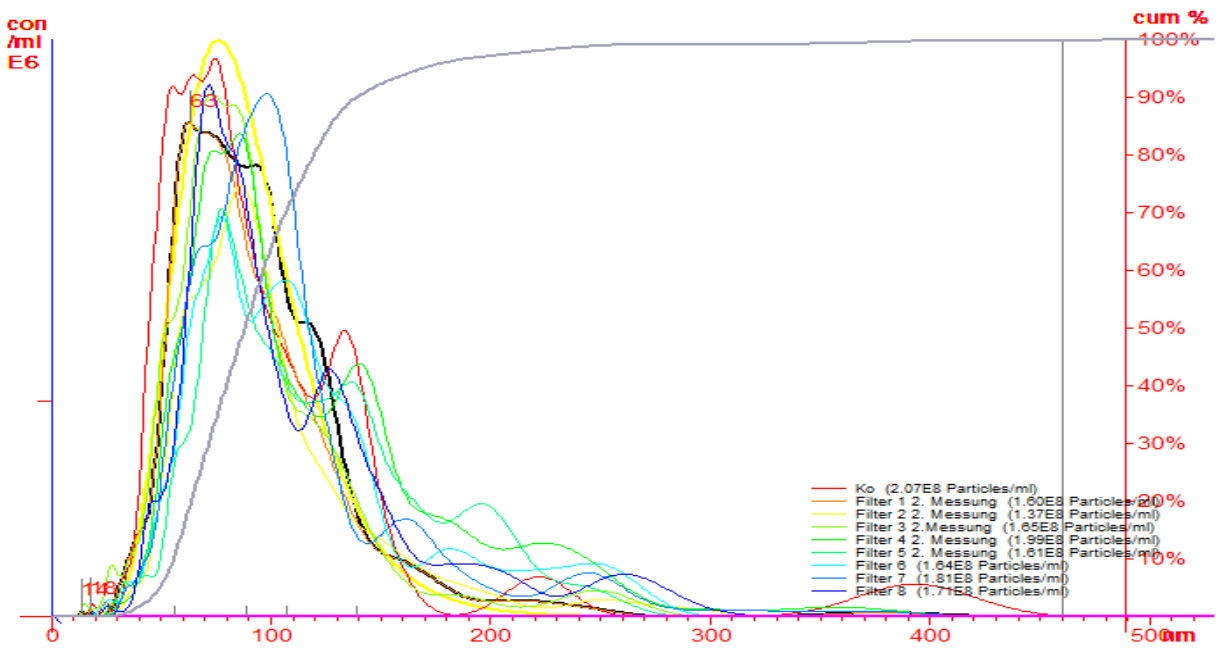

Figure V-6: Line chart of plasma vesicle size of plateletpheresis concentrates

PC samples on day $4(n=3)$ and day $7(n=4)$ were analyzed with the NTA-system NS500 (NanoSight, UK) at dilutions of 1:500.

The analysis was carried out by flow cytometry with immunolabeling of platelets and plateletderived vesicles showing PL-EV values which are comparable to other systems ( $C V=6.2 \%)$ on day 4 only. Differences in PL-EV levels were due to the discrimination of smaller vesicles in the micro flow system. Nevertheless, for the purpose of a routine analysis, the amount of PL-EVs within PCs on day 4 can be estimated by both flow cytometers Navios ${ }^{\mathrm{TM}}$ and Apogee.

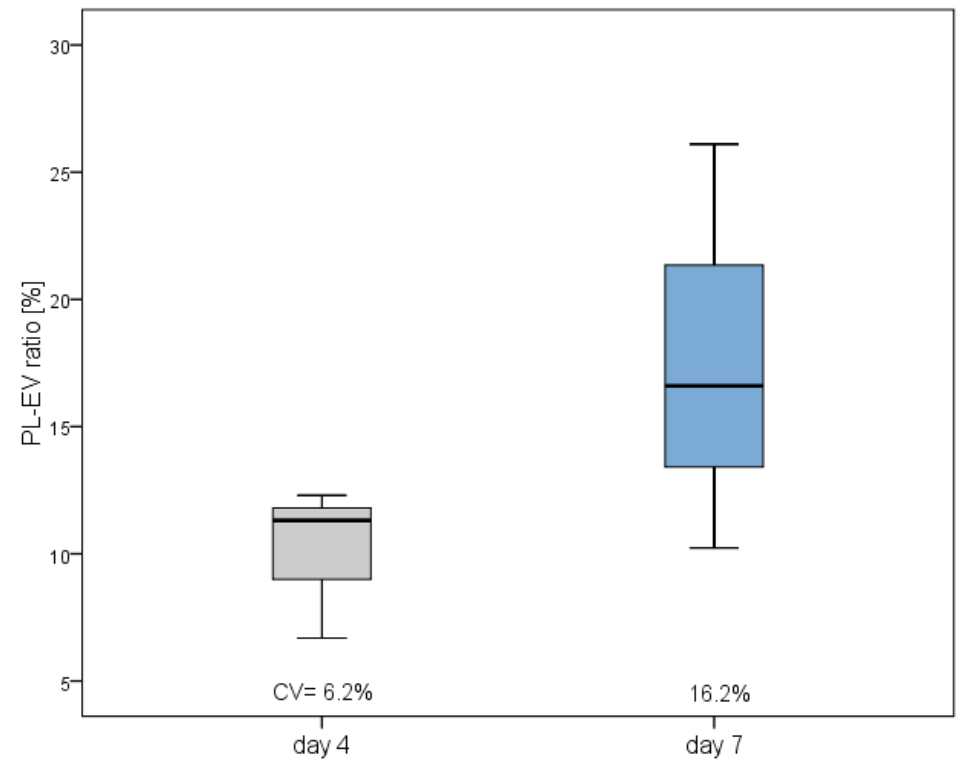

Figure V-7: Inter-assay coefficients of variability

Grey bar demonstrates the results of the PL-EV analysis on day 4, blue bar corresponds to the analysis on day 7. Navios ${ }^{\mathrm{TM}}$ (Beckman Coulter), Canto ${ }^{\mathrm{TM}}$ II (Becton Dickinson) and A50-Micro Flow Cytometer (Apogee Flow Systems Ltd., UK) were applied for the examination. 


\subsubsection{PL-EV-boost after TRAP-6 activation}

TRAP-6 activation of fresh platelets from PCs results in elevated levels of CD61 positive PLEVs. The increase reaches up to $26.4 \%$ as compared to control. Detection of PL-EVs revealed the presence of PL-EVs in a size corresponding to smaller than $500 \mathrm{~nm}$ beads measured by standard flow cytometry (sd-FCM) with Navios ${ }^{\mathrm{TM}}$ (Beckman Coulter). Incubation with PBSDulbecco, w/o $\mathrm{Ca}^{2+} / \mathrm{Mg}^{2+}$ Buffer shows no vesiculation effect of platelets. Increase of PL-EV correlates with the p-selectin surface exposure on platelets as an activation marker after stimulation.

One of the PCs showed more vesiculation as the other two PCs, because of the physiological inter-individual differences among blood donors. The mean of initial values of PL-EVs was $10.2 \%$ (the values were ranging from $7.6-11.8 \%$ in control). However, stimulated platelets show PL-EV values ranging from $10.2 \%$ to $13.8 \%$ (mean 13.3\%). Interestingly, the PC with the lowest PL-EV level before activation was most prone to activation among all PCs resulting in the highest PL-EV level after stimulation.

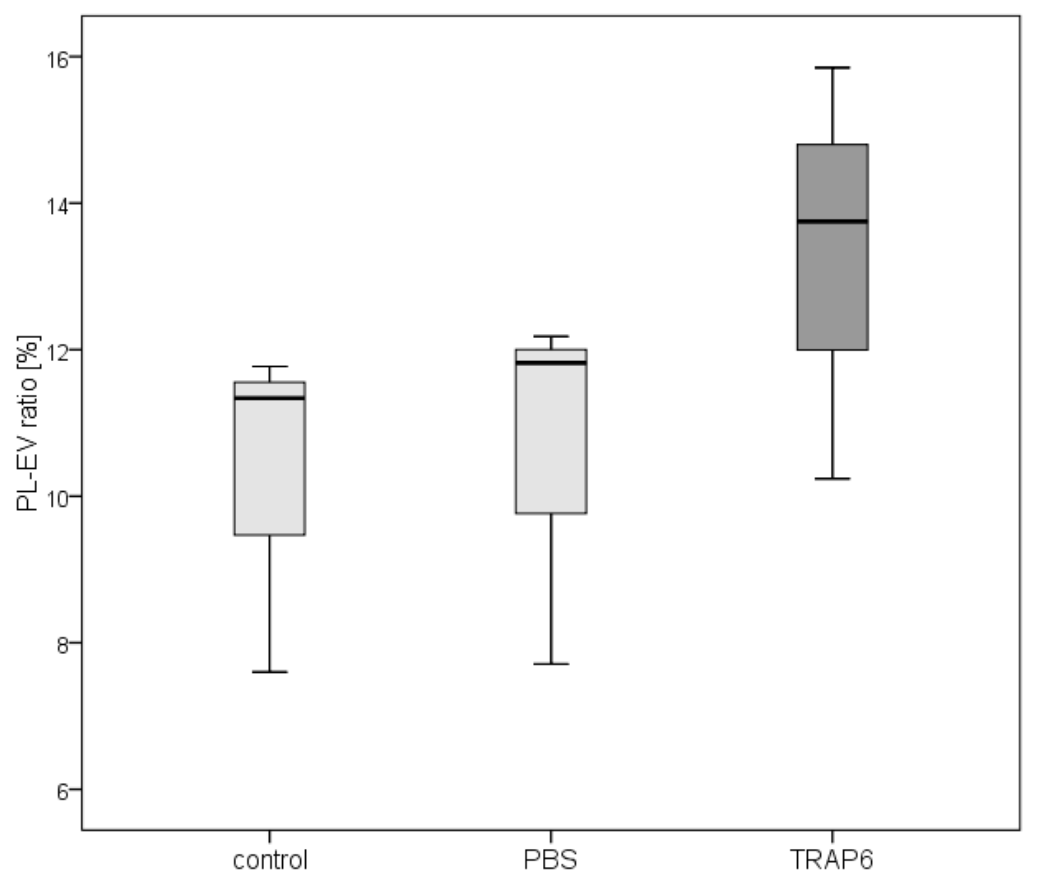

Figure V-8: Release of PL-EVs in response to TRAP-6 in fresh PCs on day 0

PL-EVs are depicted as grey box plots, marked with moAb CD61-PC7, in correlation to the activation with TRAP-6 versus control groups without stimulation. Control groups reflect native values of PL-EV production from platelets in fresh PCs. PBS-Dulbecco does not induce release of PL-EVs. Mean increase of PL-EV after TRAP- 6 stimulation against the control group reached $26.4 \%$ (ranging from 16.3 to $32.8 \%$ ). No statistically significant differences between control and TRAP-6-stimulated group (Wilcoxon, $p=0.1$ ) were found. 


\subsubsection{Rebound phenomenon, dependent on type of sampling and shear-stress}

Subsequently, it was investigated whether the initiation of platelet vesiculation and CD62P expression are reversible after resting of platelets at room temperature in sample tubes (shown in Figure V-9 and Figure V-10).

This study employed samples subjected to minimal shear stress (titled 'without shear stress') and samples subjected to strong shear stress (titled 'with shear stress') during sampling. Differences of altered vesiculation and CD62P externalization in the two sample types were investigated.

PL-EV values of both sample types reached their maxima four hours after sampling and then decreased (Figure V-9, A). Statistically significant changes over time were only computed for PL-EVs from samples subjected to shear stress. Statistical significant differences of PL-EVs between the two groups are rejectable.

24 hours after sampling the lowest CD62P values were observed for both groups, which, however, gradually elevated after 144 hours (Figure V-9, B). It is supposed that platelets subjected or not subjected to shear stress are capable of reestablishing their functionality.

Further, no statistically significant differences after 144 hours were found between the two groups, indicative that all platelets are able to sediment after hours and regain their functionality, despite no standard storage conditions were given. 

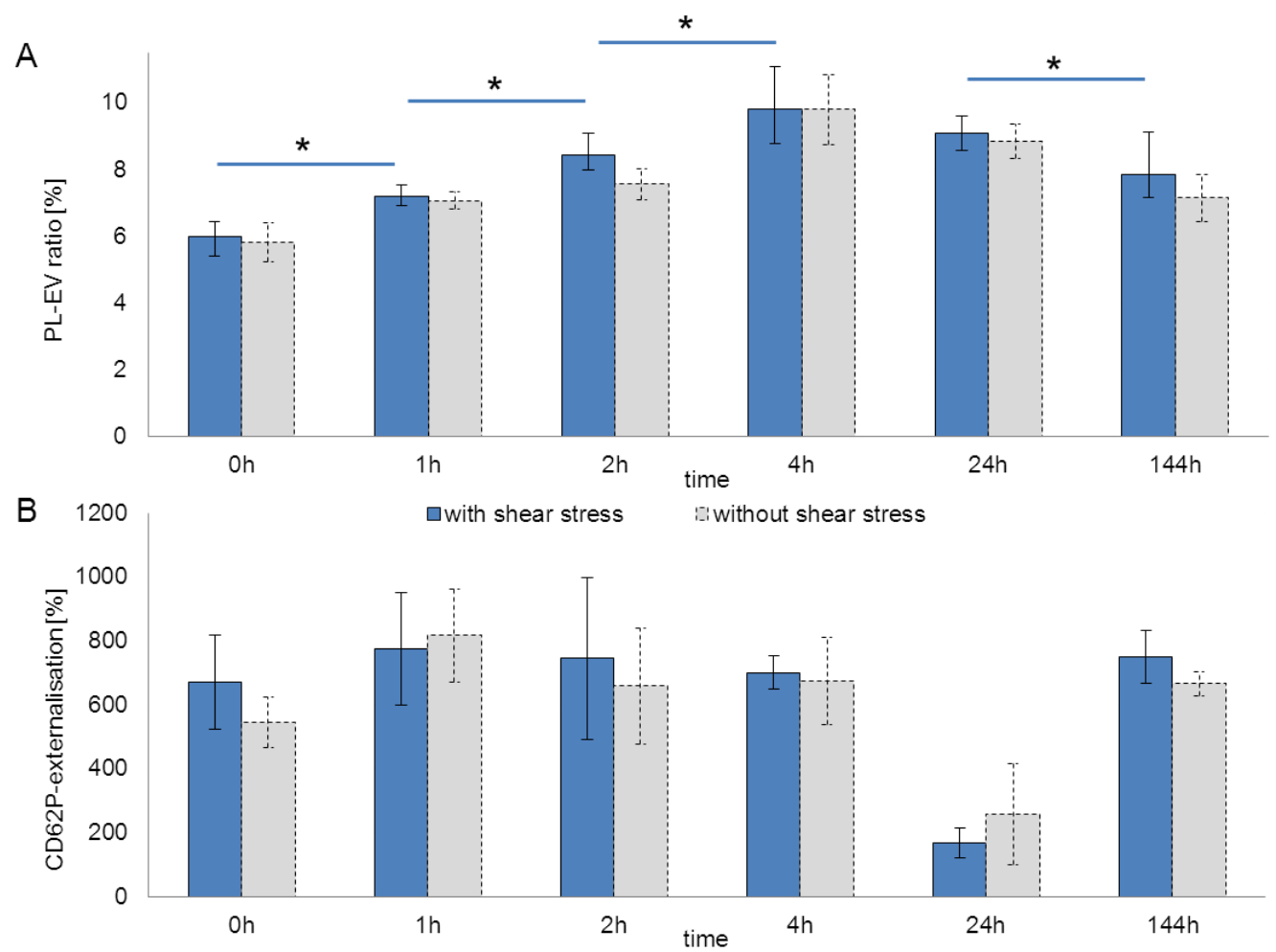

Figure V-9: Rebound effect of platelet vesiculation and platelet function over time

A: PL-EV level in PCs after activation by shear stress versus control (without shear stress). Significant differences in PL-EV amount over time were observed only among samples subjected to shear stress (Wilcoxon; $\mathrm{p}<0.05{ }^{*}$ ). B: CD62P expression on platelets after activation by shear stress versus control. In both groups, no statistically significant differences among samples over time were computed. No statistically significant differences between the two groups, neither for PL-EV nor for CD62P were determined (Mann-Whitney-U-test; $p>0.05$ ).

When correlating PL-EV-ratio and CD62P, differences within each group and differences between both groups over time occurred, that are shown in Figure $\mathrm{V}-10$.

A strong negative coherence between PL-EVs and CD62P is assumable, but only significant for samples exposed to stress at 4 hours after sampling.

The amount of PL-EVs translates to the ability of platelets to undergo activation. During the course of time the negative correlation between the presence of more PL-EVs and lower externalization of $p$-selectin upon stimulation may reflect a low quality of PCs with stressed platelets. 


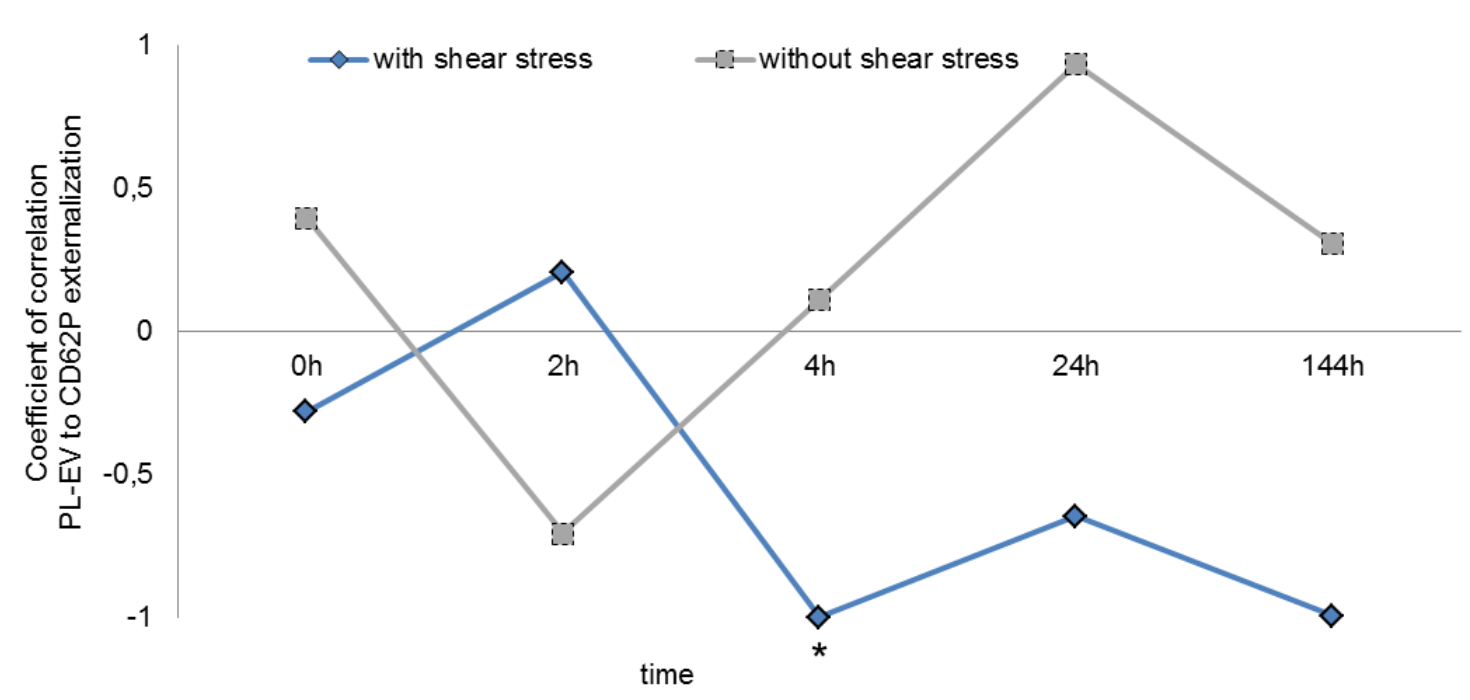

Figure V-10: Differences in rebound phenomenon depending on shear stress

In the line chart, the correlation coefficients (cc) between PL-EVs and CD62P of PC samples at various times after sampling are given, computed by Pearson's correlation (two-way-tailed, with assumed significance of $\left.0.05^{\star}\right)$. The grey line and grey dots are related to samples without shear stress, the blue line and blue dots represent samples of the same PCs with shear stress. All samples were collected manually by different type of sampling. Positive cc (data above the zero line) let assume that the more PL-EVs are measurable, the more CD62P, as a marker of activation of platelets, is expressed. Under sampling conditions without shear stress, PL-EVs are formed in a physiological way. For samples with shear stress negative cc (data below the zero line) was computed $(4-144 \mathrm{~h})$. PL-EVs formed under shear stress correlate with loss of platelet function.

For platelets without shear stress, a positive correlation between platelet activation (CD62P) and PL-EV level at the time of sampling (Oh) and after 4-144 hours was found. The effects of exhaustion of platelets, that means maximal secretion of a-granules and expression of CD62P, was reached at 2 hours after sampling and disappeared and base line values of activation response was found after 4 to $144 \mathrm{~h}$.

Opposite results were analyzed in samples subjected to shear stress. At base line, the depletion of platelet granules, shown as a negative correlation between PL-EVs and CD62P expression, was followed by a short resting after 2 hours. PC sampling under shear stress condition revealed negative correlation between PL-EVs and CD62P after 4 to 144 hours, without reaching base line values.

The increase of PL-EVs significantly correlate to lowered CD62P response on platelets (4h, $\left.c c=0.99^{*}, p=0.024\right)$. Shear stress as a platelet activator affected PL-EV response by up to $9.8 \%$ (mean; SD: $1.3 \%$ ) as compared to PL-EV level from platelets, not subjected to shear stress, which reached level of $5.1 \%$ (mean; SD: $0.6 \%$ ). 


\subsubsection{Aggregometry}

To demonstrate the effect of PL-EVs on coagulation, platelet free plasma (PFP) and plateletderived exosomes were analyzed for platelet aggregation as AUC upon TRAP-6 activation using the Multiplate $\AA$ platelet function analyser. The results show a significant aggregation difference between control (PL-EV free plasma) and PFP. Isolated exosomes (the smallest EV fraction) from PCs were added and a considerable elevation of aggregation of platelets could be detected, see Figure V-11. It is suggested, that spiked PL-EV stimulated platelet aggregation. Therefore, this batch method is not appropriate to analyze platelet aggregation in PCs resembling a mixture of platelets and procoagulant PL-EVs that cannot be discriminate.
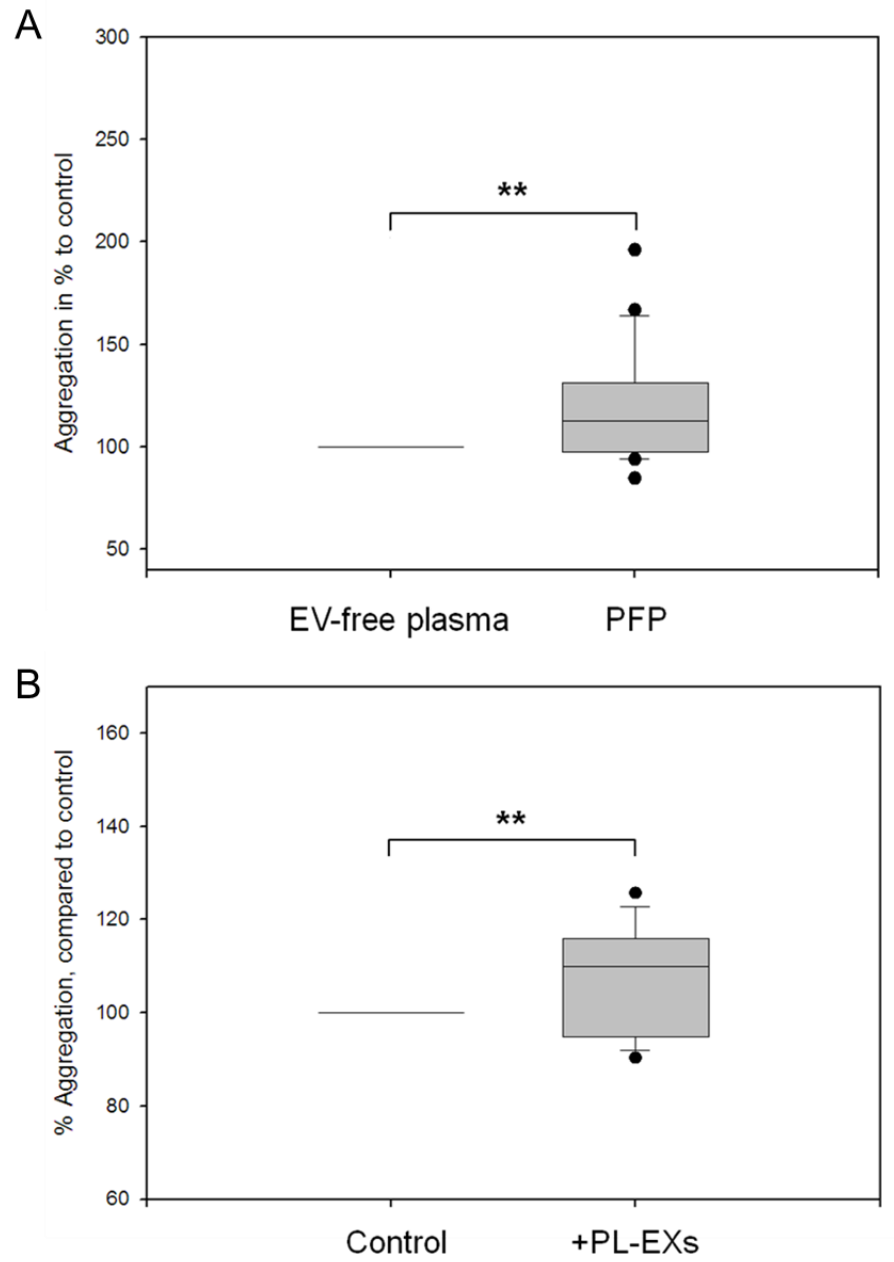

Figure V-11: Effects of PL-EVs on platelet aggregation

Both figures show the results of platelet aggregation analysis of PC-samples by TRAP-6 test using Multiplate ${ }^{\circledR}$ platelet function analyzer. A: PC-samples were prepared as EV-free plasma as control (left) versus EV-containing platelet free plasma (PFP, right). B: Analysis was also performed with PBS as control (left) versus isolated PL-exosomes (PL-EXs, right). Aggregation results, shown as area under the curve (AUC in \%) were influenced due to the fact, that PL-EVs and PL-EXs have coagulant properties. Altogether, 21 fresh PCs were used for this test. 


\subsection{Donor and apheresis specific values}

The intake of medication by donors before apheresis was excluded. All donors were healthy and no interrupts of donation were recorded. Table V-2 summarizes donor characteristics. Results of blood count (see Table V-3) and serum parameters (see Table V-4) from donors show no deviation from reference levels. All healthy volunteers classified to the study were normal weight, with normal blood pressure and with age ranging from 20 to 62 years. Due to the premenopausal fluctuation of blood count, i.e. alteration in hemoglobin and hematocrit values in women, mostly male donors were selected. No gender-related differences in the amount of PLEVs from PCs were found, not for PL-EVs measured by sd-FCM or by hs-FCM and not for determination of size/ concentration measured by NTA (Mann-Whitney-U test, $p>0.05$ ).

It is important to mention, that in the group of healthy volunteers $17 \%$ of donors showed elevated $\mathrm{Lp}$ (a) level exceeding $30 \mathrm{mg} / \mathrm{dL}$ (up to $78.3 \mathrm{mg} / \mathrm{dL}$ ), yet without any influence on the quality-defining parameters of PCs.

Oral substitution of calcium (on average $633.3 \mathrm{mg}, \mathrm{n}=15$ ) during apheresis may negatively affect the amount of PL-EVs on the day of apheresis before irradiation ( $c c=-0.5)$ and after irradiation ( $c c=-0.5$ ). Citrate consumption during donation (on average of $419.8 \mathrm{ml}, \mathrm{n}=15$ ) did not affect the level of substituted calcium ( $c c=0.4, n=15)$, but may affect the PL-EVs from freshly irradiated PCs $(\mathrm{cc}=-0.5, \mathrm{n}=15)$ and irradiated $\mathrm{PCs}$ on day $5(\mathrm{cc}=-0.59, \mathrm{n}=15)$.

Table V-2: Characteristics of donors and of the apheresis process

106 healthy donors with normal anamnesis volunteered for the study. Donor specific variables, conceived before plateletpheresis (b. p.) and after plateletpheresis (a. p.) are displayed. All data, accepted sex and apheresis system ( $\left.{ }^{*}\right)$, are reported as the mean \pm SD (range).

\begin{tabular}{ll}
\hline Variable & Value \\
\hline Sex* & 27 females/ 79 males \\
Age (years) & $34 \pm 10.6(20-62)$ \\
BMI & $24.47 \pm 2.7(18.6-31.9)$ \\
RR systolic, b. p. & $126 \pm 13.1(97-160)$ \\
RR diastolic, a. p. & $78 \pm 8.5(61-107)$ \\
RR systolic, b. p. & $116 \pm 10.9(94-145)$ \\
RR diastolic, a. p. & $74 \pm 6.9(60-93)$ \\
Pulse (per minute), b. p. & $68 \pm 10.8(47-105)$ \\
Pulse (per minute), a. p. & $71 \pm 10.0(43-101)$ \\
Duration of apheresis & $59.3 \pm 10.9(40-95)$ \\
Processed blood volume & $3397.3 \pm 545.0(2263-4814)$ \\
Apheresis system by Amicus (double-needle system) ${ }^{*}$ & 68 \\
Apheresis system by Trima (single-needle system) & 38 \\
\hline
\end{tabular}


Table V-3: Blood count parameter of platelet donors

Automated hematological analysis of specimens from 106 healthy donors, obtained within 4 hours after drawing blood samples by XE5000 (Sysmex). HGB: hemoglobin, HCT: hematocrit, RBC: red blood cells, RDW-SD: red cell distribution width as standard deviation from the mean red cell size, RDW-CV: red cell distribution width as a coefficient of variation from the mean red cell size. PLT: platelets, PDW: platelet distribution width, MPV: mean platelet volume, IPF: immature platelet factor. All values are reported as the mean \pm SD (range).

\begin{tabular}{lll}
\hline Variable & Value & Reference level \\
\hline HGB [g/ dL] & $14.4 \pm 0.9(12.3-17.1)$ & $11.2-15.7$ \\
HCT [\%] & $42.2 \pm 2.5(36.9-48.2)$ & $34.1-44.9$ \\
RBC [10 $/ \boldsymbol{\mu L}]$ & $4.8 \pm 0.4(3.9-5.8)$ & $3.93-5.22$ \\
RDW-SD [fL] & $42.1 \pm 2.6(36.9-50.9)$ & $36.4-46.3$ \\
RDW-CV [\%] & $13.1 \pm 0.7(11.9-15.6)$ & $11.7-14.4$ \\
PLT [10 $/ \mu L]$ & $264.6 \pm 38.4(186-373)$ & $182-369$ \\
PDW [fL] & $11.7 \pm 1.5(8.5-18)$ & $9.8-16.2$ \\
MPV [fL] & $10.2 \pm 0.7(8.6-12.8)$ & $9.4-12.5$ \\
IPF [\%] & $2.6 \pm 1.3(0.6-9.6)$ & $1.1-6.1$ \\
IPF [nL] & $6.8 \pm 3.4(1.4-19.3)$ & - \\
\hline
\end{tabular}

Table V-4: Serum characteristics of donors

Analysis of serum blood samples of donors was carried out with automated laboratory analyzer by photometry, nephelometry, immunturbidometry, ELISA, CLIA, coagulometry, chromogenic method. Modified number of samples is displayed in the last slot (n). CHOL: total cholesterol, LDL-C: low density lipoprotein cholesterol, HDL-C: high density lipoprotein cholesterol, TRIG: triacylglycerol, $\operatorname{Lp}(\mathrm{a})$ : lipoprotein (a), VLDL-C: very low density lipoprotein cholesterol, Apo E: apolipoprotein E, ApoB100: apolipoprotein B100, ApoB48: apolipoprotein B48, Apo A-I: apolipoprotein A-I, Apo C-I: apolipoprotein C-I, Apo-J: apolipoprotein J (also known as clusterin), GLC: blood glucose, HBA1c: hemoglobin A1-C, IGF-1: insulin-like growth factor 1, IGFPB3: insulin-like growth factor binding protein 3, CRP: c-reactive protein, C3c: complement factor c3, ATIII: antithrombin 3, PAI-1: plasminogen activating inhibitor 1. All values are reported as the mean $\pm S D$ (range).

\begin{tabular}{llll}
\hline Variable & Value & Reference level & $\mathbf{n}$ \\
\hline CHOL $[\mathrm{mg} / \mathbf{d L}]$ & $186.4 \pm 33.4(113-280)$ & $<200$ & 81 \\
LDL-C $[\mathrm{mg} / \mathbf{d L}]$ & $109.6 \pm 29.6(55-186)$ & $<100$ & 81 \\
HDL-C [mg/ dL] & $54.6 \pm 13.4(29-88)$ & $40.0-60.0$ & 81 \\
TRIG $[\mathrm{mg} / \mathbf{d L}]$ & $133.0 \pm 57.8(47-346)$ & $<150$ & 81 \\
Lp(a) $[\mathrm{mg} / \mathbf{d L}]$ & $19.9 \pm 18.5(5.0-78.3)$ & $<30$ & 80 \\
VLDL-C [mg/ dL] & $21.2 \pm 8.5(9.0-52.0)$ & $0.0-40.0$ & 35 \\
Apo E $[\mathbf{m d} / \mathbf{d L}]$ & $3.4 \pm 1.0(1.83-8.44)$ & $2.2-6.3$ & 46 \\
Apo B100 [mg/ dL] & $84.1 \pm 20.4(50.3-140.0)$ & $55.0-125.0$ & 35
\end{tabular}


continuation of Table V-4: Serum characteristics of donors

\begin{tabular}{|c|c|c|c|}
\hline Variable & Value & Reference level & $\mathbf{n}$ \\
\hline Apo B48 $[\mu \mathrm{g} / \mathrm{mL}]$ & $17.1 \pm 14.6(4.1-91.6)$ & $3.53-7.29$ & 35 \\
\hline Apo A-I [mg/ dL] & $156.9 \pm 21.0(124.0-212.0)$ & $125.0-215.0$ & 47 \\
\hline Apo C-I $[\mu \mathrm{g} / \mathrm{dL}]$ & $4.4 \pm 0.9(3.02-6.51)$ & $4.0-7.0$ & 35 \\
\hline Apo $\mathrm{J}[\mu \mathrm{g} / \mathrm{mL}]$ & $53.8 \pm 10.3(37.3-78.5)$ & $80.5-165.0$ & 35 \\
\hline GLC $[\mathrm{mg} / \mathrm{dL}]$ & $78.3 \pm 29.9(12.0-165.0)$ & $74.0-106.0$ & 48 \\
\hline HBA1c [NGSP] & $5.2 \pm 0.2(4.8-5.7)$ & $4.8-5.9$ & 35 \\
\hline HBA1c [IFCC] & $33.6 \pm 2.4(29.0-39.5)$ & $29.0-42.0$ & 35 \\
\hline IGF-1 [ng/ mL] & $174.2 \pm 47.7(95-330)$ & $93.5-251.9$ & 35 \\
\hline IGFBP3 [ $\mu \mathrm{g} / \mathrm{mL}]$ & $4.6 \pm 0.7(3.3-6.3)$ & $3.4-6.7$ & 35 \\
\hline CRP [mg/ L] & $2.9 \pm 0.1(2.9-3.8)$ & - & 62 \\
\hline $\mathrm{C} 3 \mathrm{c}[\mathrm{mg} / \mathrm{dL}]$ & $105.4 \pm 17.2(72.7-154.0)$ & $90.0-180.0$ & 47 \\
\hline Fibrinogen [mg/ dL] & $262.4 \pm 35.9(194.1-347.3)$ & $210.0-400.0$ & 35 \\
\hline ATIII [\%] & $99.3 \pm 9.0(82.9-115.1)$ & $75.0-125.0$ & 35 \\
\hline PAl-1 [U/ mL] & $1.1 \pm 1.1(0.0-3.9)$ & $2.0-7.0$ & 35 \\
\hline
\end{tabular}

Table V-5 presents several relevant donor parameters, which were selected in order to compare them with the quality determining parameters of PCs. The age of donors did not significantly correlate to the expression of $\mathrm{CD} 62 \mathrm{P}$ in fresh platelets. A positive correlation between $\mathrm{BMI}$ and CD62P is likely to occur both on day 0 and on day 5 . A negative correlation of BMI to the amount of PL-EVs on day 5 was observed. This allows assumption, that the higher the BMI level, the lower PL-EV levels are detectable on day 5. Duration of apheresis expectedly affects the platelet count in PCs and further the expression of CD62P on platelets and the amount of PL-EVs on day 0. Two correlations were found to be the most essential: the first one relates to the application of single-needle against double-needle system in correlation to CD62P expression (Figure V-12). The second one relates to different apheresis instruments correlating to PL-EV levels (Figure V-13). It is worth notifying that the single-needle system requires less time in combination with higher flow rates and higher levels of citrate, which in turn lowers the $\mathrm{pH}$, thus affecting the activation of platelets. This induced considerable differences in vesiculation of fresh platelets obtained with the use of different apheresis instruments. It was also observed that the higher levels of immature platelet fraction (IFP) of donors, obtained before apheresis, revealed lower PL-EV levels detected on day 0 and higher levels on day 5. 
Table V-5: Relevant correlation of donor-specific, clinical or laboratory parameters to PC quality

\begin{tabular}{|c|c|c|c|c|c|c|c|c|}
\hline \multirow{4}{*}{$\begin{array}{l}\text { Parameter of } \\
\text { donors }(n=42)\end{array}$} & \multicolumn{8}{|c|}{ Quality parameter of plateletpheresis concentrates $(n=42)$} \\
\hline & \multicolumn{8}{|c|}{ Correlation coefficient (bold) and significance (Pearson, two-way analysis) } \\
\hline & \multicolumn{5}{|c|}{ day 0} & \multicolumn{3}{|c|}{ day 5} \\
\hline & 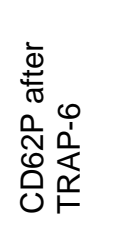 & 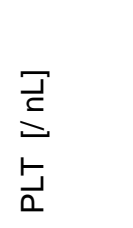 & 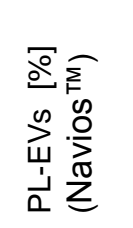 & 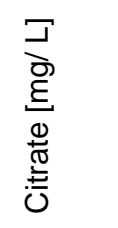 & $\begin{array}{l}00 \\
\frac{0}{D} \\
\frac{0}{\pi} \\
\frac{1}{2} \\
\frac{1}{2}\end{array}$ & 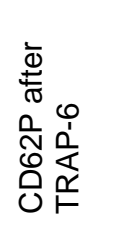 & $\begin{array}{l}\vec{\Xi} \\
\Xi \\
\sqcup \\
\square\end{array}$ & 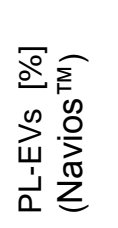 \\
\hline $\begin{array}{l}\text { single-needle/ } \\
\text { double-needle } \\
\text { system }\end{array}$ & $\begin{array}{l}-0.575^{\star *} \\
0.000\end{array}$ & - & - & $\begin{array}{l}\mathbf{0 . 4 1 2 ^ { \star }} \\
0.024\end{array}$ & $\begin{array}{l}-0.327^{\star} \\
0.035\end{array}$ & $\begin{array}{l}-0.412^{\star \star} \\
0.007\end{array}$ & 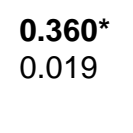 & - \\
\hline Age [years] & $\begin{array}{l}0.315^{*} \\
0.042\end{array}$ & - & - & - & - & - & - & - \\
\hline BMI & 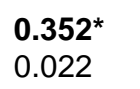 & - & - & - & - & $\begin{array}{l}\mathbf{0 . 4 2 3} 3^{\star *} \\
0.005\end{array}$ & - & $\begin{array}{l}-0.316^{\star} \\
0.041\end{array}$ \\
\hline $\begin{array}{l}\text { Apheresis } \\
\text { instrument }\end{array}$ & $\begin{array}{l}\mathbf{0 . 3 6 6 ^ { * }} \\
0.017\end{array}$ & - & $\begin{array}{l}-0.456^{*} \\
0.011\end{array}$ & - & - & 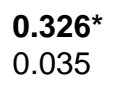 & $\begin{array}{l}\mathbf{0 . 3 3 5 ^ { \star }} \\
0.03\end{array}$ & $\begin{array}{l}-0.369^{\star} \\
0.016\end{array}$ \\
\hline $\begin{array}{l}\text { Duration of } \\
\text { apheresis }\end{array}$ & $\begin{array}{l}\mathbf{0 . 2 3 0} 0^{\star} \\
0.039\end{array}$ & $\begin{array}{l}0.406^{\star \star} \\
0.008\end{array}$ & $\begin{array}{l}\mathbf{0 . 4 1 7}^{\star} \\
0.022\end{array}$ & - & - & - & - & - \\
\hline IPF $[/ n L]$ & - & & $\begin{array}{l}-0.429^{*} \\
0.018\end{array}$ & - & - & - & $\begin{array}{l}0.308^{\star} \\
0.048\end{array}$ & $\begin{array}{l}\mathbf{0 . 4 2 2} 2^{\star \star} \\
0.005\end{array}$ \\
\hline $\begin{array}{l}\text { Cholesterol } \\
{[\mathrm{mg} / \mathrm{dL}]}\end{array}$ & $\begin{array}{l}\mathbf{0 . 3 4 6 ^ { * }} \\
0.025\end{array}$ & - & - & - & - & - & - & - \\
\hline $\begin{array}{l}\text { LDL cholesterol } \\
{[\mathrm{mg} / \mathrm{dL}]}\end{array}$ & 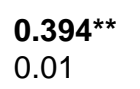 & - & - & - & - & - & - & - \\
\hline
\end{tabular}

Cholesterol level, especially LDL-Cholesterol, showed a weak but positive correlation to activation sensitivity (CD62P) of platelets on day 0 . Other donor- or apheresis-related parameters show no significant association with the quality determining parameters of PCs. 


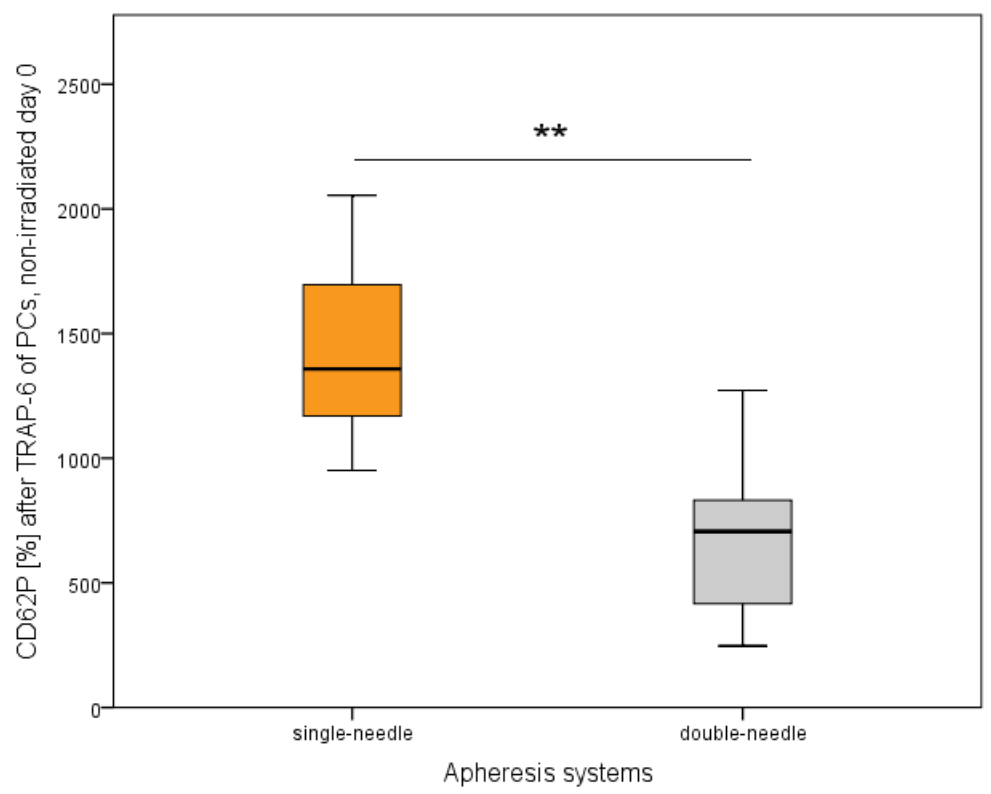

Figure V-12: Effect of single-needle versus double-needle apheresis system on CD62P

Box plots represent CD62P expression on fresh platelets obtained by two different apheresis systems. The correlation of CD62P expression to apheresis systems is significantly negative for the benefit of the single-needle system (Pearson two-way analysis, $\left.p=-0.575^{\star \star}\right)$.

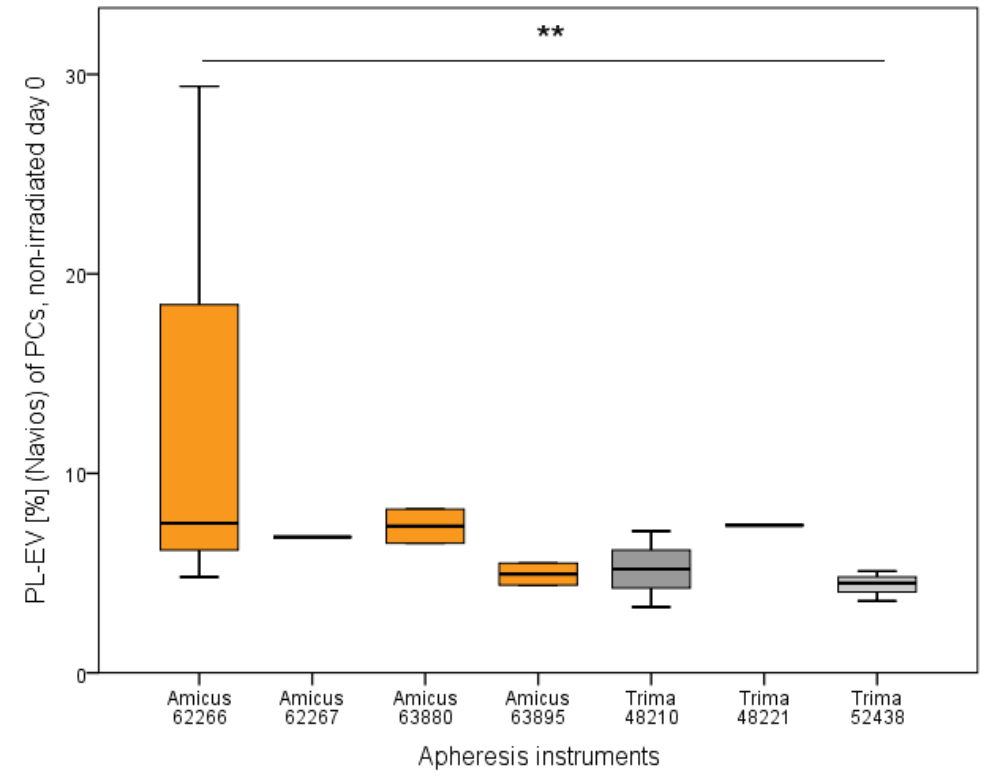

Figure V-13: Effect of apheresis instruments on PL-EV levels in PCs

Differences in the values of PL-EVs on day 0 before irradiation among the treatment groups (apheresis instruments) are greater than expected. There is a statistically significant difference between the results obtained with the help of the first instrument (A62266) and the results originating from the other instruments $(p=0,026$; Kruskal-Wallis one-way analysis of variance on ranks).The instruments were applied with the same frequency. 
BMI and age of the donors (Table V-5) correlate significantly positive to evaluated serum lipids, such as triglycerides (TRIG) and HDL-cholesterol (HDL-C, for BMI only), cholesterol (CHOL) and LDL-cholesterol (LDL-C, both for age only) and complement protein C3c (for BMI only).

Age of the donors correlated equally significant to apolipoprotein (Apo) B-100 and to the level of $\mathrm{HbA1c}$ (according to NGSP or IFCC).

As shown in Table V-6, a considerable correlations were found between TRIG and Apo E levels, constituents of remnants of chylomicrons and VLDL; and between HDL-C and Apo A-I, components of HDL particles. CHOL and LDL-C negatively correlated to glucose levels before apheresis, reflecting differences of the fasting or postprandial state of donors early in the morning.

Associations were found among red blood cells (RBC), hemoglobin (HGB) and hematocrit (HCT) on the one hand and among HDL-C, C3c (HGB and HCT only) and Apo A-I (RBC only) on the other hand. The RBC count and Apo A-I levels inversely correlated to higher concentration of RBC, which in turn corresponds to lower Apo A-I levels. In contrast, HDL-C levels positively correlated to RBC count. Positive correlation between RBC and HCT on one side and HDL-C on the other side was observed. However, HGB negatively correlated to HDLC. Complement proteins, in this case $\mathrm{C} 3 \mathrm{c}$, are known to bind to RBC under several conditions. According to results obtained in the current study, no positive correlation between C3c and RBC was found, but C3c correlated positively to HGB and HCT.

Evaluated donor-related serum parameters (not shown in the table) did not match any quality control defining parameters for PCs. 
Table V-6: Relevant donor specific parameters in correlation to one another

\begin{tabular}{|c|c|c|c|c|c|c|c|c|}
\hline \multirow{3}{*}{$\begin{array}{l}\text { Parameter of } \\
\text { donors }(n=42)\end{array}$} & \multicolumn{8}{|c|}{ Parameter of donors $(n=42)$} \\
\hline & \multicolumn{8}{|c|}{ Correlation coefficient (bold) and significance (Pearson, two-way analysis) } \\
\hline & 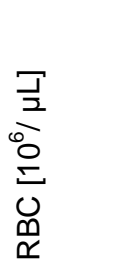 & $\begin{array}{l}\text { ㅁ } \\
\text { ర) } \\
\text { 0 } \\
\text { 조 }\end{array}$ & 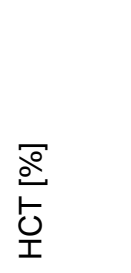 & 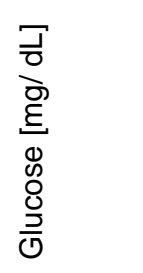 & $\bar{\Sigma}$ & 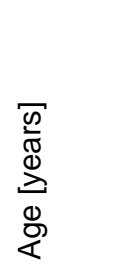 & $\begin{array}{l}\overline{1} \\
8 \\
\frac{8}{4}\end{array}$ & $\begin{array}{l}\text { ш } \\
\stackrel{\circ}{<}\end{array}$ \\
\hline Triglycerides & - & - & - & - & $0.332^{\star \star}$ & - & - & $0.745^{\star \star}$ \\
\hline$[\mathrm{mg} / \mathrm{dL}]$ & & & & & 0.002 & & & 0.009 \\
\hline Cholesterol & - & - & - & $-0.723^{\star \star}$ & - & $0.486^{\star \star}$ & - & - \\
\hline$[\mathrm{mg} / \mathrm{dL}]$ & & & & 0.008 & & 0.001 & & \\
\hline LDL-C & - & - & - & $-0.816^{\star \star}$ & - & $0.530^{\star \star}$ & - & - \\
\hline$[\mathrm{mg} / \mathrm{dL}]$ & & & & 0.001 & & 0.000 & & \\
\hline HDL-C & $0.631^{\star \star}$ & $-0.531^{\star \star}$ & $0.584^{\star \star}$ & - & $0.473^{\star \star}$ & & $0.84^{\star \star}$ & - \\
\hline$[\mathrm{mg} / \mathrm{dL}]$ & 0.000 & 0.000 & 0.000 & & $0-002$ & - & 0.001 & \\
\hline Apo A-I & $\begin{array}{l}-0.624^{\star} \\
0.03\end{array}$ & - & - & - & - & - & - & - \\
\hline ApoB100 & - & - & - & - & - & $\begin{array}{l}\mathbf{0 . 5 2 0} \\
0.001\end{array}$ & - & - \\
\hline $\mathrm{C} 3 \mathrm{c}$ & - & $\begin{array}{l}0.755^{\star \star} \\
0.005\end{array}$ & $\begin{array}{l}\mathbf{0 . 6 4 8} \\
0.023\end{array}$ & - & $\begin{array}{l}0.408^{\star *} \\
0.004\end{array}$ & - & - & - \\
\hline $\begin{array}{l}\mathrm{HbA1c} \\
\text { (NGSP) }\end{array}$ & - & - & - & - & - & $\begin{array}{l}\mathbf{0 . 5 1 9} \mathbf{A}^{\star *} \\
0.001\end{array}$ & - & - \\
\hline $\begin{array}{l}\mathrm{HbA1c} \\
\text { (IFCC) }\end{array}$ & - & - & - & - & - & $\begin{array}{l}\mathbf{0 . 5 2 0}{ }^{\star \star} \\
0.001\end{array}$ & - & - \\
\hline
\end{tabular}




\subsection{Platelet concentrate specific values}

\subsubsection{Characteristics of PCs over time}

In this study, the platelet count, immature platelet factor (IPF), citrate concentration in PCs, pH values and typical expression markers on the surface of platelets were evaluated, as well as the measurement of platelet-derived extracellular vesicles (PL-EVs) and plasma vesicles. The values obtained on day 0 and day 5 , together with the number of cases, are reported in Table V-7 and Table V-8.

The effects of sampling, irradiation and storage on the parameters are presented in the following chapters. The coherence of PL-EVs and plasma vesicles to the expression of $\mathrm{p}$ selectin, used here as a main characteristic of platelet viability, are presented separately (5.3.6 et seqq.).

Table V-7: Characteristic laboratory analysis of PCs over time

All values are reported as the mean \pm SD (range). Reference levels pertain to the standard test and acceptance certificates in plateletpheresis manufacturing ( $\dagger$ only on day 0 ; $\ddagger$ only on day 5 ; PC: samples from the whole PC; Tube: samples from tubing system).

\begin{tabular}{|c|c|c|c|c|c|}
\hline Variable & day 0 & $\mathbf{n}$ & day 5 & $\mathbf{n}$ & $\begin{array}{l}\text { Reference } \\
\text { level }\end{array}$ \\
\hline $\begin{array}{l}\text { PLT } \\
{\left[10^{3} / \mu L\right]}\end{array}$ & $\begin{array}{l}1180.2 \pm 324.6(9-1639) \\
1268.5 \pm 142.5(946-1639) \mathrm{PC} \\
1087.6 \pm 424.7(9-1533) \text { Tube }\end{array}$ & $\begin{array}{l}82 \\
42 \\
40\end{array}$ & $1284.3 \pm 180.2(849-1780)$ & 66 & $800-1600 \ddagger$ \\
\hline IPF [\%] & $1.8 \pm 0.9(0.4-3.8)$ & 41 & $3.4 \pm 1.6(1.0-7.3)$ & 25 & - \\
\hline $\begin{array}{l}\text { Citrate } \\
{[\mathrm{mg} / \mathrm{L}]}\end{array}$ & $3213.5 \pm 412.9(2554-4230)$ & 30 & - & - & - \\
\hline $\mathrm{pH}$ & $7.2 \pm 0.02(7.14-7.27)$ & 42 & $7.35 \pm 0.08(7.0-7.5)$ & 42 & $6.4-7.8 \dagger$ \\
\hline \multirow{3}{*}{$\begin{array}{l}\text { CD61- } \\
\text { PerCP [\%] }\end{array}$} & $141.7 \pm 19.3(98-182)$ & 69 & \multirow[t]{3}{*}{$142.1 \pm 35.3(25-221)$} & \multirow[t]{3}{*}{50} & \multirow[t]{3}{*}{-} \\
\hline & $146.5 \pm 18.6(98-177) P C$ & 30 & & & \\
\hline & $138.0 \pm 19.3(104-182)$ Tube & 39 & & & \\
\hline \multirow{3}{*}{$\begin{array}{l}\text { CD41- } \\
\text { PE [\%] }\end{array}$} & $188.9 \pm 40.6(104-349)$ & 68 & \multirow[t]{3}{*}{$187.9 \pm 97.3(73-802)$} & \multirow[t]{3}{*}{50} & \multirow[t]{3}{*}{-} \\
\hline & $194.9 \pm 42.3(104-349) P C$ & 30 & & & \\
\hline & $184.2 \pm 39.1(114-275)$ Tube & 38 & & & \\
\hline \multirow{3}{*}{$\begin{array}{l}\text { CD62P. } \\
\text { FITC [\%] }\end{array}$} & $1094.7 \pm 767.0(121-4232)$ & 81 & \multirow[t]{3}{*}{$547.3 \pm 294.8(103-1613)$} & \multirow[t]{3}{*}{62} & \multirow[t]{3}{*}{-} \\
\hline & $1328.3 \pm 775.4(288-4232) \mathrm{PC}$ & 42 & & & \\
\hline & $843.1 \pm 681.6(121-2737)$ Tube & 39 & & & \\
\hline
\end{tabular}




\subsubsection{Platelet-derived extracellular vesicles and storage}

The values of PL-EV ratio obtained by sd-FCM and hs-FCM on day 0 and day 5 as well as nonirradiated versus irradiated PCs are listed in Table V-8.

The table summarizes PL-EV events per $\mu \mathrm{L}$ both for all PL-EVs and for individual subpopulations (small, medium, large), discriminated by hs-FCM, in addition to platelet count, plasma vesicle concentration and plasma vesicle size distribution by NTA.

Plasma vesicles measured by NTA in fresh, non-irradiated PCs occurred in a concentration of $5.9687 \times 10^{8} / \mathrm{mL}$ (mean), whereas irradiated fresh PCs reached the concentration of 6.2753 $\mathrm{x} 10^{8} / \mathrm{mL}$ (mean). In irradiated PCs after expiry, the plasma vesicle concentration was evaluated at $6.06 \times 10^{8} / \mathrm{mL}$ as measured by NTA.

The medium sized PL-EVs (180 nm to $300 \mathrm{~nm}$ ) represented the major subfraction of all PL-EVs regardless of the duration of analysis constituting $57-58 \%$ of the population.

Flow cytometric quantification with the Apogee A-50 Micro showed that PL-EVs occurred in fresh, non-irradiated PCs at a concentration of 1633.75/ $\mu \mathrm{L}$ (mean) and decreased after irradiation to a concentration of 1359.03/ $\mu \mathrm{L}$ (mean). PL-EV events per $\mathrm{mL}$ from irradiated PCs after expiry appeared at a mean concentration of $2754.3 / \mu \mathrm{L}$.

The platelet count determined by Apogee A-50 Micro was sufficiently comparable to the platelet count measured by Sysmex automated hematology analyzer on day $0\left(1241.9 \times 10^{3} / \mu \mathrm{L} ; 1180\right.$ $\left.\mathrm{x} 10^{3} / \mu \mathrm{L}\right)$ and on day $5\left(1408.1 \times 10^{3} / \mu \mathrm{L} ; 1284.3 \times 10^{3} / \mu \mathrm{L}\right)$. 
Table V-8: PL-EV and plasma vesicle values in PCs analyzed by flow cytometry and NTA

The semiquatitative PL-EV-ratio [\%] was measured by the Navios ${ }^{\mathrm{TM}}$ system $\left({ }^{\circ}\right)$ with samples in a final dilution of 1:2500. The PL-EV-ratio [\%] and, further, the quantitative PL-EV analysis were performed with A-50 Micro (†) and plasma vesicle analysis by NTA with NS-500 (*) with the same samples in a final dilution of 1:500 [non-irrad.: non-irradiated; irrad.: irradiated; PC: samples from the whole PC; Tubes: samples from the PC tubing system].

\begin{tabular}{|c|c|c|c|c|c|}
\hline Variable & & day 0 & $\mathbf{n}$ & day 5 & $\mathbf{n}$ \\
\hline \multicolumn{6}{|l|}{ PL-EVs by FCM } \\
\hline \multirow[t]{3}{*}{ PL-EV ${ }^{\circ}$ non-irrad. } & \multirow{6}{*}{ 운 } & $6.1 \pm 3.7(2.4-17.4) P C$ & 15 & $7.1 \pm 3.1(3.1-18.7)$ & 27 \\
\hline & & $6.69 \pm 5.13(2.4-29.4) P C$ & 30 & & - \\
\hline & & $8.8 \pm 10.4(2.78-61.7)$ Tubes & 40 & - & - \\
\hline PL-EV ${ }^{\circ}$ irrad. & & $6.8 \pm 5.6(3.3-26.7) P C$ & 15 & $\begin{array}{l}- \\
11.8 \pm 5.8(4.4-24.3) \\
P C\end{array}$ & 22 \\
\hline PL-EV † non-irrad. & & $67.0 \pm 65(12-236)$ & 15 & - & - \\
\hline PL-EV † irrad. & & $54.0 \pm 61(8-235)$ & 15 & $97.0 \pm 63(19-213)$ & 15 \\
\hline Small PL-EV † non-irrad. & \multirow{10}{*}{ 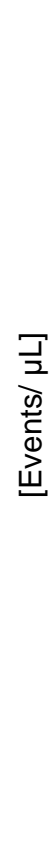 } & $55.08 \pm 53.77(10-212)$ & 15 & - & - \\
\hline Small PL-EV † irrad. & & $29.11 \pm 15.26(12-63)$ & 15 & $\begin{array}{l}164.4 \pm 222(18.5- \\
886.0)\end{array}$ & 15 \\
\hline Medium PL-EV † non-irrad. & & $951.27 \pm 1020.38(113-3687)$ & 15 & - & - \\
\hline Medium PL-EV † irrad. & & $794.53 \pm 951.33(61-3580)$ & 15 & $\begin{array}{l}1574.2 \pm 1169.2(160 \\
3459)\end{array}$ & 15 \\
\hline Large PL-EV † non-irrad. & & $627.4 \pm 664.25(162-2760)$ & 15 & - & - \\
\hline Large PL-EV † irrad. & & $535.4 \pm 618.13(116-2557)$ & 15 & $\begin{array}{l}1015.7 \pm 749.3(33- \\
2944)\end{array}$ & 15 \\
\hline Platelet count $†$ non-irrad. & & $2483.8 \pm 345.4(1568-2864)$ & 15 & - & - \\
\hline Platelet count † irrad. & & $2658.33 \pm 351.63(1956-3281)$ & 15 & $\begin{array}{l}2816.2 \pm 673.1(1699- \\
3920)\end{array}$ & 15 \\
\hline All PL-EV † non-irrad. & & $1633.75 \pm 1688.62(324-6589)$ & 15 & - & - \\
\hline All PL-EV † irrad. & & $1359.03 \pm 1555.15(233-6161)$ & 15 & $\begin{array}{l}2754.3 \pm 1958.3(508.5 \\
-7246)\end{array}$ & - \\
\hline \multicolumn{6}{|l|}{ Plasma vesicles by NTA } \\
\hline Concentration * non-irrad. & 豆 & $5.9687 \pm 2.99(2.28-11.96)$ & 15 & - & - \\
\hline Concentration * irrad. & 㞾 & $6.2753 \pm 3.27(2.48-13.02)$ & 15 & $\begin{array}{l}6.06 \pm 2.49(2.75- \\
11.08)\end{array}$ & 15 \\
\hline Size distribution * non-irrad. & 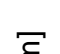 & $150.2667 \pm 16.45(124-175)$ & 15 & - & - \\
\hline Size distribution * irrad. & $\stackrel{\text { E }}{\subseteq}$ & $150.4667 \pm 19.39(129-201)$ & 15 & $\begin{array}{l}157.53 \pm 19.65(129- \\
194)\end{array}$ & 15 \\
\hline
\end{tabular}




\subsubsection{Comparability of sample types (PC samples and tube samples)}

Considering the fact that there may be alternative sampling methods for quality control analysis using residual samples from the tubes of the apheresis system on day 0 , we tested whether samples from the tubing system are comparable with samples from the whole PCs. The equivalence for platelet count was rejected, because of the high variance of platelet count, especially for the lower platelet count in samples from the tubing system, see Figure V-14.

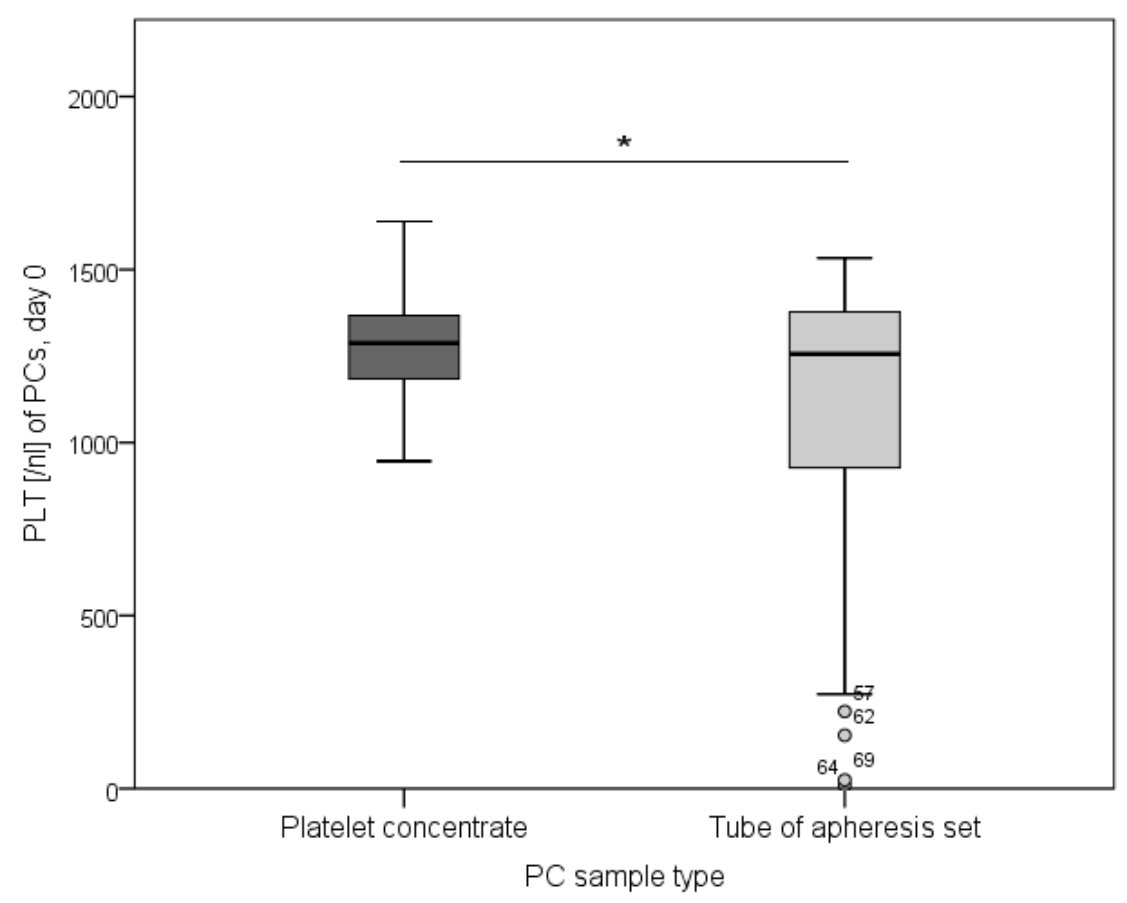

Figure V-14: Box plots for platelet count values of two sample types of plateletpheresis quality control analysis

PC samples $(n=42)$ and tube samples $(n=40)$ were compared; both groups were non-irradiated. Dark horizontal lines represent the mean within the boxes corresponding to the $25^{\text {th }}$ and $75^{\text {th }}$ percentiles and the whiskers depict the $5^{\text {th }}$ and $95^{\text {th }}$ percentiles (outliers represented by circles). Statistical analysis was performed with Mann-Whitney-U-test, $p=0.284$. In addition, the two groups were statistically analyzed for assumed equality of platelet count with an equivalencetest (TOST: two-one-sided-test, XLSTAT Excel) based on supposition that the groups are normally distributed. $90 \%$ confidence interval of the mean $(\alpha=0.05$, confidence interval $(1-2 x$ $0,05) \times 100=90 \%$ ) was used to explore the difference and to compare it to the defined TOST interval of upper bound and lower bound with $255 \mathrm{PLT} / \mathrm{nL}$, according to $20 \%$ of PLT/ $\mathrm{nL}$ value in standard samples (PC samples from the whole PC content). It is noticeable that at the total ${ }^{*} p$-value of 0.803 ( $p$ is not 0.005 for equality), the equivalence is rejected. 
In addition, the equivalence of both sampling methods has to be rejected since the expression of $p$-selectin (CD62P) on platelets (Figure V-15) is differentially distributed between PCs and tubes which show significant lower expression of $p$-selectin.

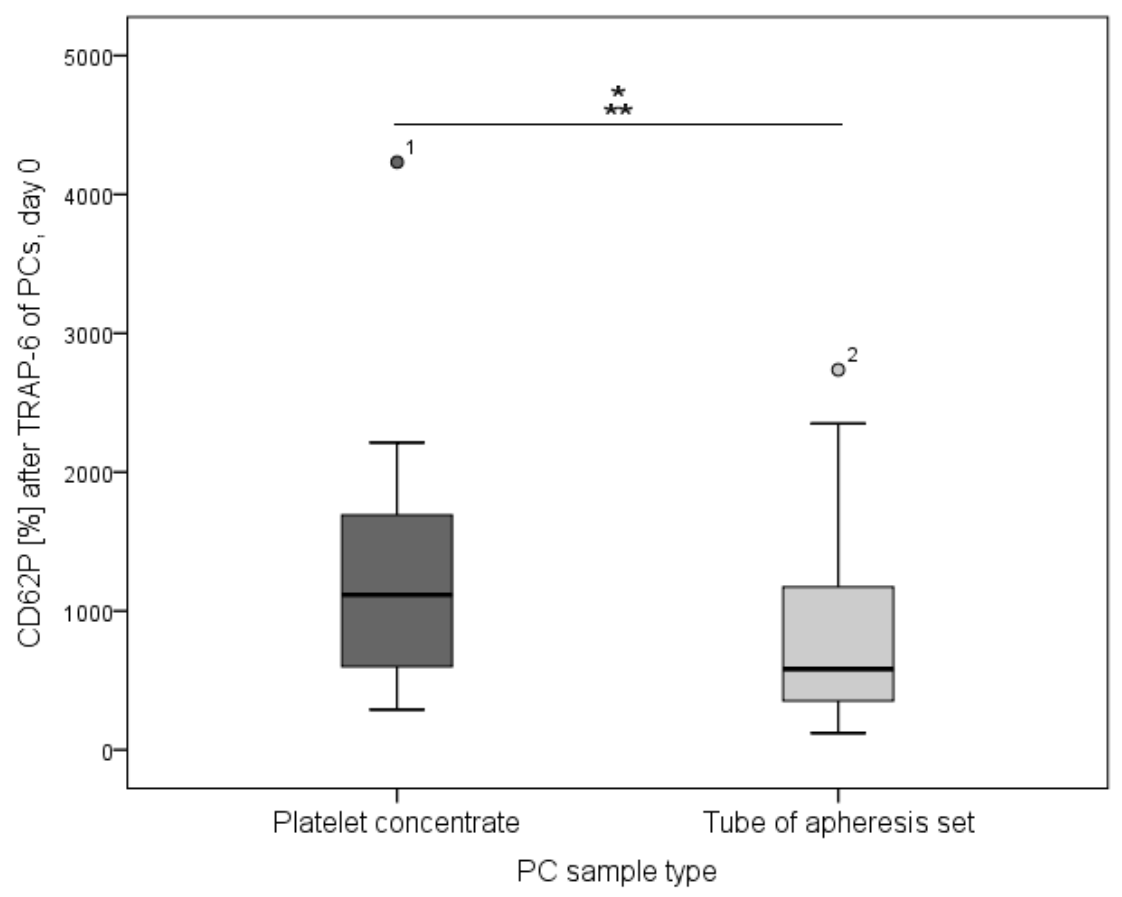

Figure V-15: Box plots for CD62P expression on platelets from PCs of two sample types

Samples of whole plateletpheresis concentrates $(n=42)$ versus samples of PC-filled tubes $(n=39)$, were compared for the analysis of immunolabeled platelets after stimulation with TRAP6 . The values were calculated as percentage of mean fluorescent intensity (MFI) from samples without stimulation (PBS-Dulbecco), which represent 100\%. Outliers are represented by circles. Statistical analysis was performed with Mann-Whitney-U-test, $p=0.04\left({ }^{*} p<0.05\right)$. In addition, the two groups were statistically analyzed for assumed equality of CD62P expression with an equivalence-test (XLSTAT Excel) on supposition that the groups are normally distributed. $90 \%$ confidence interval of the mean ( $\alpha=0.05$, confidence interval $\left.\left(1-2^{*} 0,05\right)^{*} 100=90 \%\right)$ was used to explore the difference and compare it to the defined TOST interval of upper bound and lower bound with $260 \%$, according to $20 \%$ of CD62P expression value in standard samples (PC samples from the whole PC content). It is obvious, that at the total ${ }^{* *} p$-value of 0.881 ( $p$ is not 0.005 for equality), the comparability is not sufficient.

In QC analysis, the values referring to PL-EV amount from tubes of PCs were inconsistently distributed as compared to the values of PL-EVs of the whole PCs (see Figure V-16).

The analysis of samples taken from the tubing system of PCs did not produce equivalent results for platelet count, $p$-selectin expression on platelets and the amount of PL-EVs as compared to the samples from the whole PC. Therefore, the use of tube samples for quality control is not recommended. 


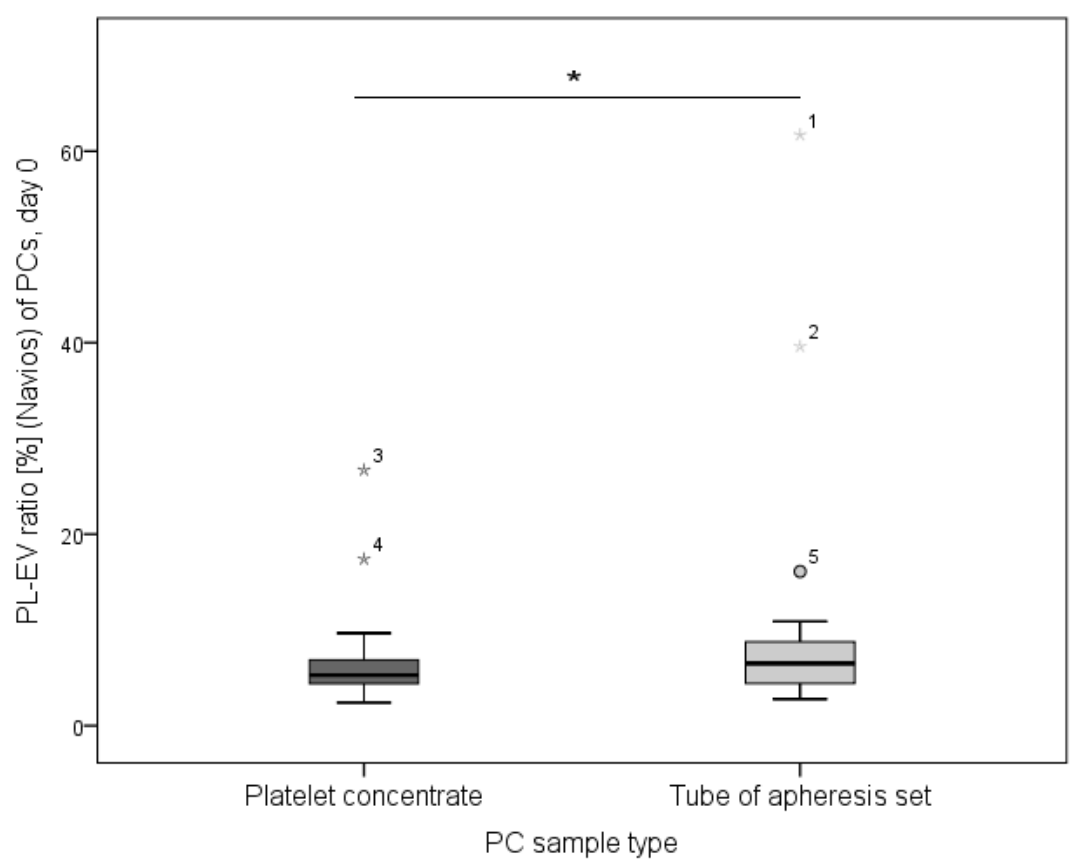

Figure V-16: Box plots of the interquartile range (IQR) for PL-EV raw data of different sample types

PC samples ( $n=30$, non-irradiated) and tube samples ( $n=40$, non-irradiated) were compared. The boxes signify the upper and lower quartiles and dark horizontal lines within the boxes represent the mean (outliers represented by circles, extreme outliers by stars). PL-EV levels in PC samples varied from 2.4 to $26.7 \%$ and in tube samples from 2.8 to $61.7 \%$. There are more extreme outliers of the tube samples than in PC samples, because of the inhomogeneity of the sample und platelet aggregates (for example, falsely determined ratio of PLT/ PL-EVs contributable to less platelet events measured by flow cytometry). Statistical analysis was performed with Mann-Whitney-U-test, $p=0.148$. In addition, the two groups were statistically analyzed for assumed equality of PL-EVs with an equivalence-test (XLSTAT Excel) on supposition that the groups are normally distributed. $90 \%$ confidence interval of the mean was used $\left(\alpha=0.05\right.$, confidence interval $\left.\left(1-2^{*} 0,05\right)^{*} 100=90 \%\right)$ to generate the difference and to compare it to the defined TOST interval of upper bound and lower bound with 1,28\%, according to $20 \%$ of PL-EV mean values in standard samples (PC samples from the whole PC content). With a total ${ }^{*} p$-value of 0.659 ( $p$ is not 0.005 for equality), the equivalence is not sufficient.

\subsubsection{Effects of irradiation (Differences between non-irradiated versus irradiated PCs)}

Counts of platelets on day 0 significantly differ $(p=0.035)$ between the two groups of nonirradiated PCs and irradiated PCs, but platelet count elevates during the gamma irradiation process (Figure V-17). Elevation of platelet count after irradiation analyzed by hs-FCM confirms this assumption (Figure V-20). No significant difference was observed for platelet count on day 5 between the two groups $(p=1.06)$. 


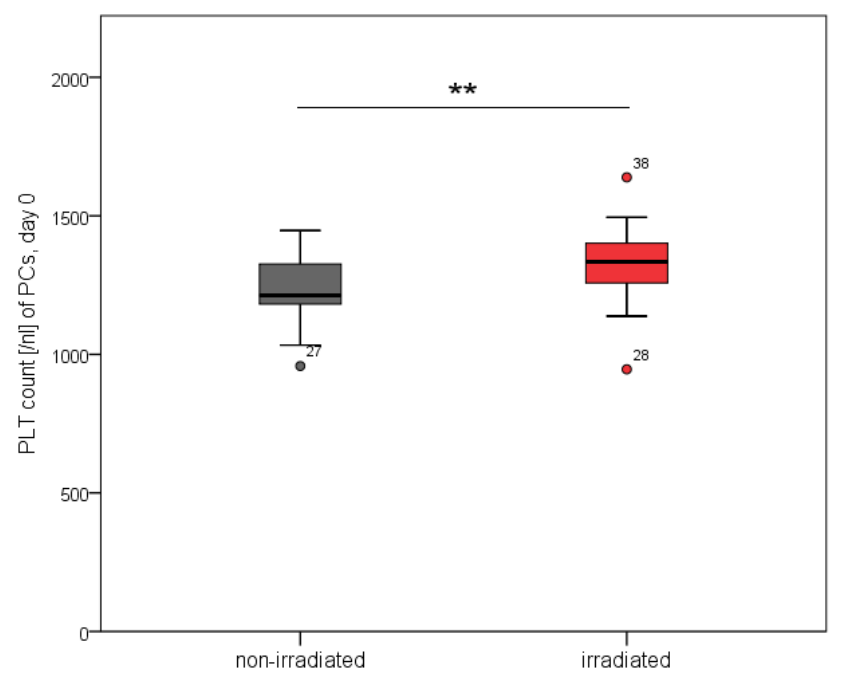

Figure V-17: Box plots illustrating the effect of irradiation on platelet count

Platelet count of plateletpheresis concentrates ( $n=42 ; 27$ non-irradiated, 15 irradiated) on day 0 were analyzed with XE-1500 (Sysmex). Statistical analysis with Mann-Whitney-tests supposes a statistically significant difference between both groups, $p=0.035^{\star \star}$. No significant difference was found in platelet count values for both groups on day $5(p=0.106)$.

The PL-EV ratio measured by sd-FCM (Navios ${ }^{\mathrm{TM}}$ ) showed strong deviations between the two groups on day $5(p=0.002)$, whereas no statistically significant PL-EV elevation measured by sdFCM on day 0 ( $p=0.56)$ was found. The PL-EV ratio visibly increased in irradiated PCs during storage; see Figure V-18.

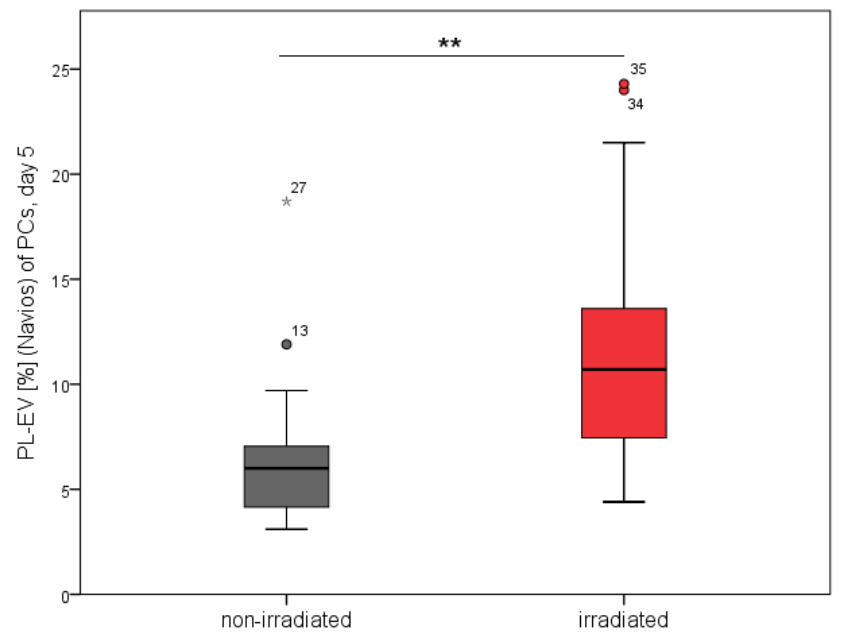

Figure V-18: Box plots illustrating the effect of irradiation on PL-EV levels measured by sd-FCM

The dissimilar shedding of platelet-derived extracellular vesicles not exposed (grey box) or exposed to irradiation (red box) is shown using PL-EV values on day 5 (Navios ${ }^{\mathrm{TM}}$, Beckman Coulter). Platelet concentrates ( $n=42 ; 27$ non-irradiated, 15 irradiated) were stored under standard blood banking conditions. Statistically significant differences within PL-EVs, measured by the Mann-Whitney test, $p=0.002^{\star *}$, were solely found on day 5 . 
These results were not confirmed by the analysis with hs-FCM (Apogee). Statistically significant differences of the PL-EV ratio on day 0 showed decreased PL-EV ratios after irradiation (Figure $\mathrm{V}-19$ and Figure $\mathrm{V}-20$ ) in contrast to the nonsignificant increase of the ratio after irradiation as determined by sd-FCM.

The PL-EV ratio depends on the platelet count in FCM. This count was evaluated by numbers of events per $\mu \mathrm{L}$ for platelets, which increased after irradiation. However, the PL-EV/ PLT ratio may decrease false positively because of the inappropriate counting of large platelets by an instrument that is configured for small particles (hs-FCM).

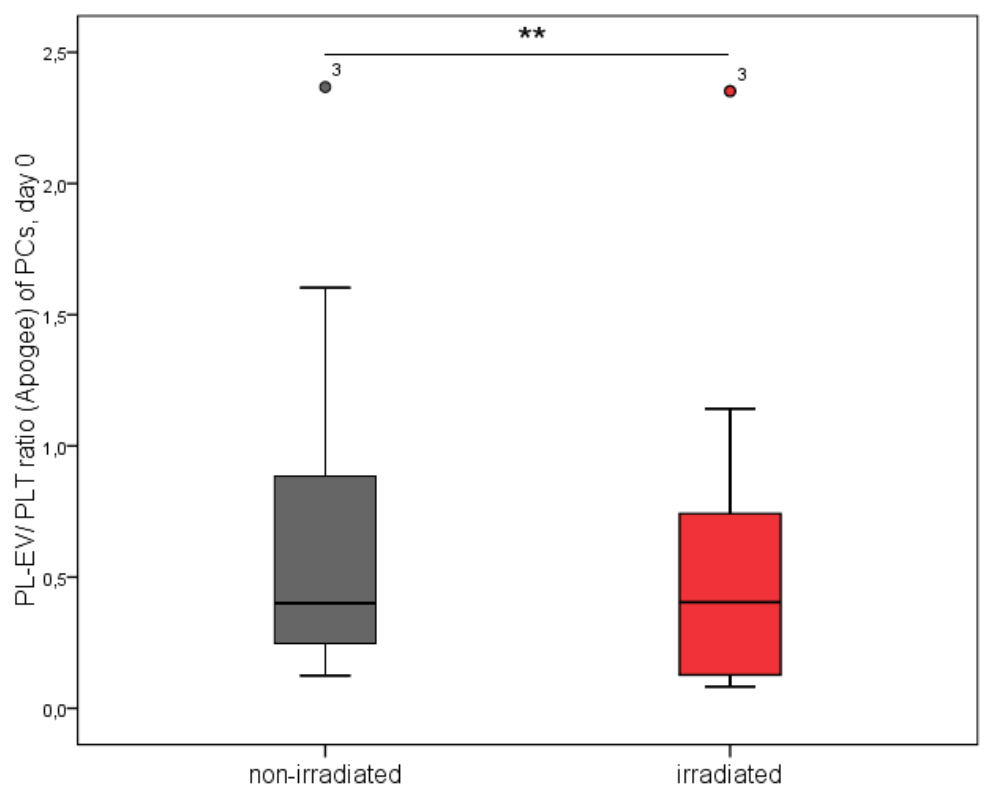

Figure V-19: Effect of irradiation on the PL-EV/ PLT ratio in fresh PCs measured by hs-FCM

The analysis was performed by hs-FCM with A50-Micro flow cytometer (Apogee Flow Systems Ltd., UK) on 15 samples of the same PCs before and after irradiation with 25 Gy. Statistically significant differences can be assumed (Wilcoxon test, $p=0.001$ ). 


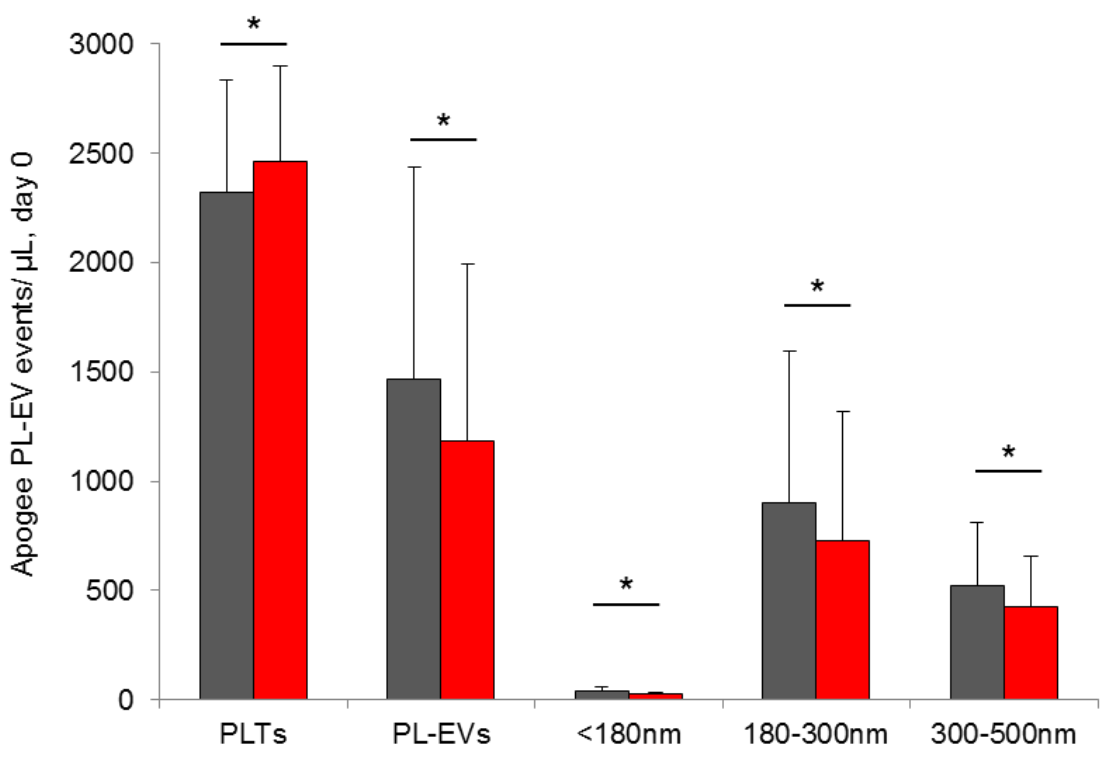

Figure V-20: Effect of irradiation on PL-EV and PLT count in PCs measured by hs-FCM

The analysis was performed with the same non-irradiated and subsequently irradiated PCs $(n=15)$ on day 0 by flow cytometry with A50-Micro flow cytometer (Apogee Flow Systems Ltd., UK). Statistically significant differences can be assumed among the samples of the same PCs before and after irradiation (Wilcoxon test, two-tailed; PLT $p=0.027^{*}$; PL-EV $p=0.02^{*}$; small PLEVs $p=0.031^{*}$; medium PL-EVs $p=0.001^{* *}$; large PL-EVs $\left.p=0.005^{\star *}\right)$.

In contrast, the values of all plasma vesicles, i.e. vesicle concentration and size distribution, evaluated by NTA did not differ between the two groups of PCs on day 0 as shown in Figure $\mathrm{V}-21$ and Figure $\mathrm{V}-22$.

There are no statistically significant differences between the two groups with non-irradiated or irradiated PCs ( $n=42$, in 15 cases irradiated) in the values of $p$-selectin expression on fresh platelets (day 0 ) found with Mann-Whitney-test: $p=0.265$ and Kruskal-Wallis-test, $p=0.265$. Further, p-selectin externalization did not exhibit any changes on day 5 (Mann-Whitney-test: $\mathrm{p}=0.59$ and Kruskal-Wallis-test, $\mathrm{p}=0.590$ ). 


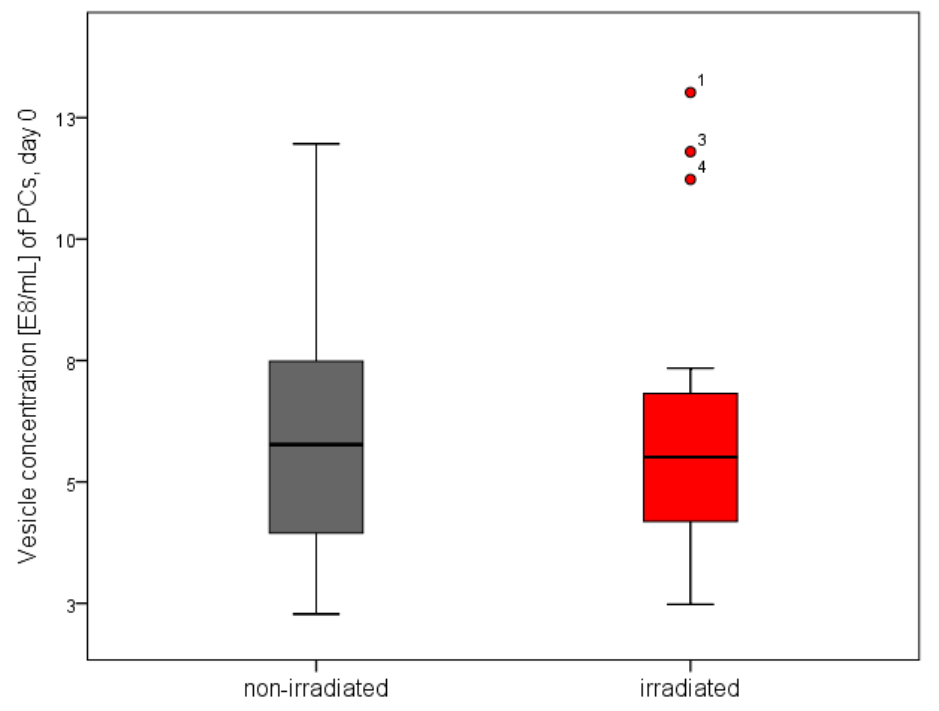

Figure V-21: Effect of irradiation on the vesicle concentration in PCs measured by NTA

The analysis was performed on 15 samples of the same PCs before and after irradiation with 25 Gy by NTA (NS500, NanoSight). No statistically significant difference can be assumed (Wilcoxon test, $\mathrm{p}=0.233$ ). There are more extreme upper outliers after irradiation, figured as red circles above the red box plot.

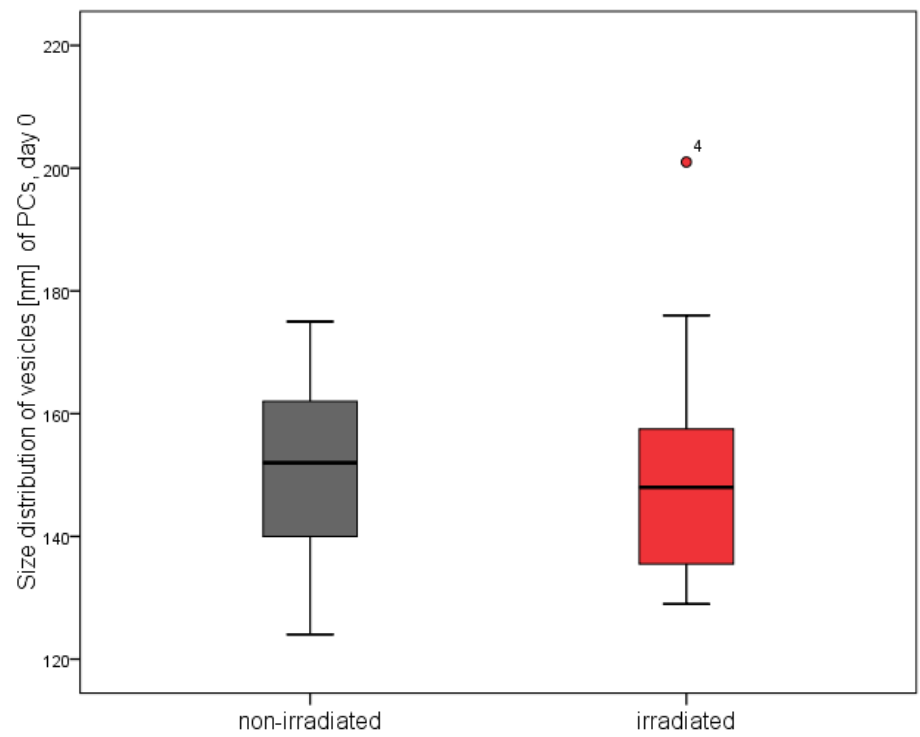

Figure V-22: Effect of irradiation on size distribution of vesicles in PCs measured by NTA

The EV analysis of PC samples before and after irradiation $(n=15)$ was performed on day 0 by NTA with NS500 (NanoSight). Statistically significant differences cannot be assumed (Wilcoxon, $\mathrm{p}=0.706)$. 


\subsubsection{Alteration of PCs during storage}

The platelet count of 42 PCs during 5 days of storage under standard blood banking conditions showed no statistically significant differences ( $p=0.573$; Figure V-23). Statistical analysis by Wilcoxon-test revealed comparable results for subgroups of non-irradiated PCs between day 0 and $5(p=0.517, n=27)$ and irradiated PCs over time ( $p=0.82, n=15$; not figured as box plots).

The expression of $p$-selectin on platelets upon stimulation differed between PCs on day 0 and PCs on day $5(p=0.000, n=42)$ as shown in Figure $V-24$, likewise in subgroups of non-irradiated versus irradiated PCs.

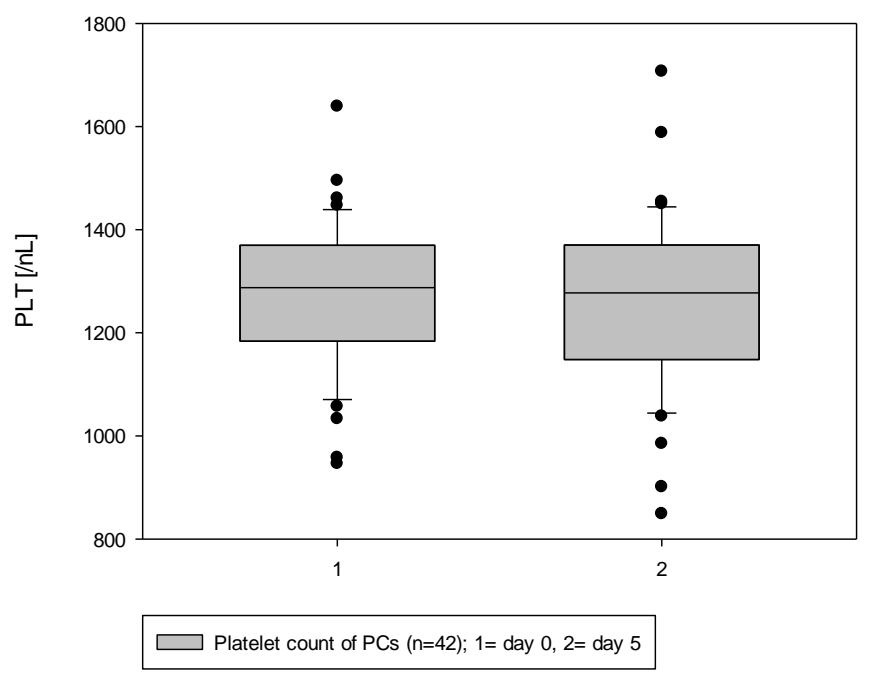

Figure V-23: Box plots demonstrating changes of platelet count over time

After analysis of PLT count (XE-1500, Sysmex) in 42 PCs on day 0 (27 non-irradiated, 15 irradiated) and continued analysis of the same PC samples on day 5, the figure corresponding to the obtained results presents no statistically significant differences between platelet count on the day of apheresis and on the day after date of expiry (statistical analysis was performed with the Wilcoxon test, $\mathrm{p}=0.573$ ). 
A

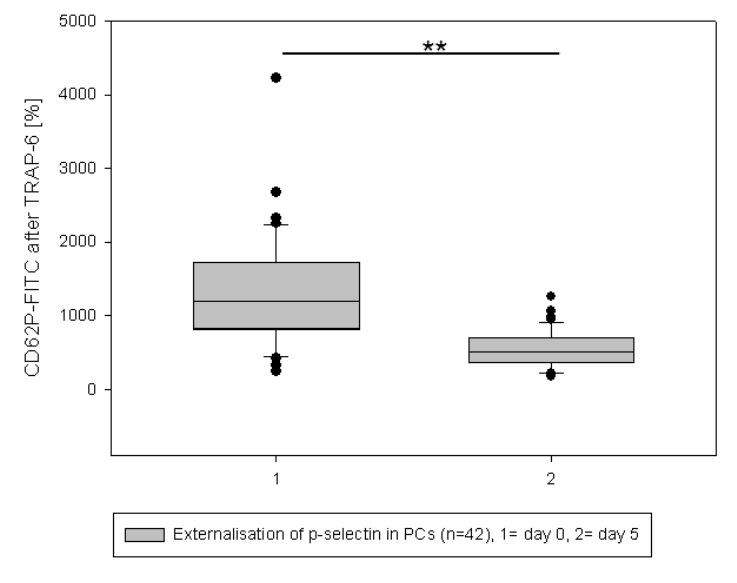

B

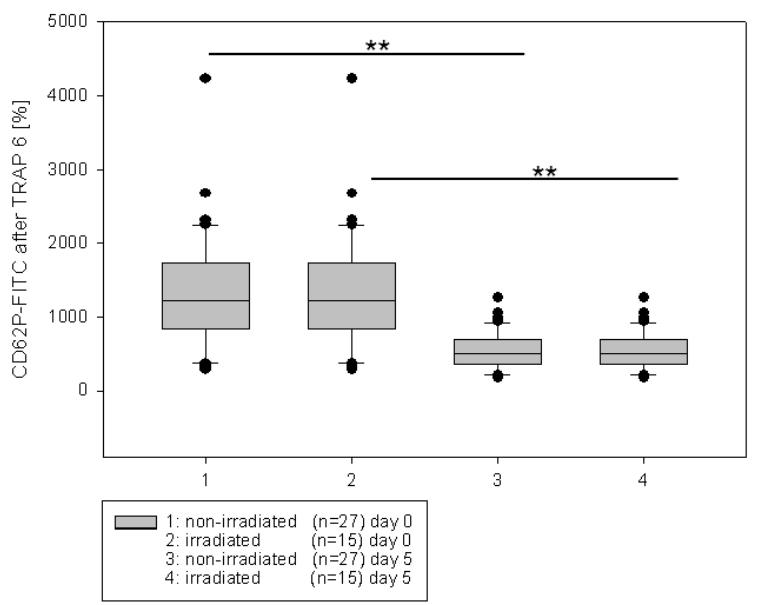

Figure V-24: Box plots of different CD62P externalization on platelets of PCs over time $(A)$ and after irradiation (B)

A: P-selectin after stimulation with TRAP-6 (platelet function test with Canto ${ }^{\mathrm{TM}} \mathrm{II}, \mathrm{BC}$ ) on day 0 and on day 5 of the same plateletpheresis concentrates (PCs, $n=42 ; 27$ non-irradiated, 15 irradiated) was evaluated. Statistical analysis was performed with the Wilcoxon test, $p=0.000^{\text {** }}$ and statistically significant differences are acceptable. B: Results of platelet function test on day 0 and day 5 showed no statistically significant inter-group differences between non-irradiated and irradiated PCs on day 0 ( 1 and 2 ) or on day 5 ( 3 and 4). Therefore, the results of intra-group comparison showed differences within non-irradiated PCs ( 1 and 3 , day 0 and day $5, p=0.000^{* *}$ ) and irradiated PCs ( 2 and 4 , day 0 and day $\left.5, p=0.001^{\star *}\right)$.

The PL-EV ratio measured by sd-FCM significantly differ between the group of PCs on day 0 [7.8 $\pm 8.4(2.4-61.7)]$ and PCs on day $5[8.6 \pm 4.7(3.1-24.3)](\mathrm{p}=0.000)$ as shown in Figure $\mathrm{V}-25$. The PL-EVs increase during the storage of PCs did not depend on irradiation on day 0. Both subgroups, non-irradiated $(p=0.009)$ and irradiated PCs $(p=0.002)$, exhibited increased levels of PL-EVs in comparison to the unaltered platelet count over time (Figure V-26).

When comparing PL-EVs from fresh PCs and PL-EVs from expired PCs, significant differences were observed. It is suggested that the higher PL-EV values on day 0 is accompanied by a higher PL-EV concentration on day 5. ( $\mathrm{cc}=0.725$; Figure V-27). The more PL-EVs were determined in fresh PCs, the more PL-EVs in PCs over time were measurable. 


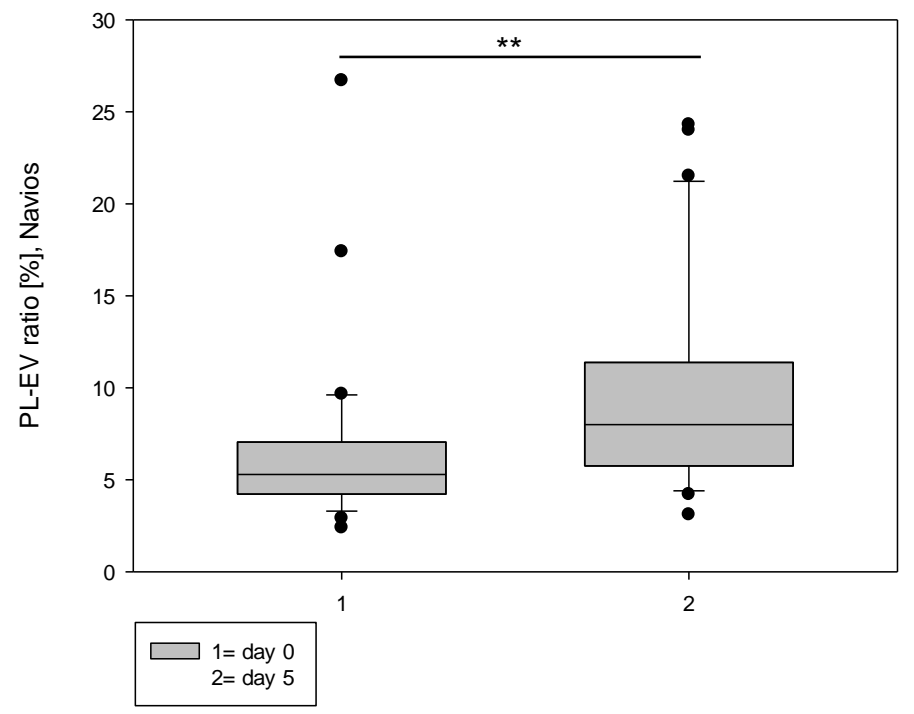

Figure V-25: Box plots of shedded PL-EVs in PCs over time measured by sd-FCM

PCs ( $n=30,15$ non-irradiated) on day 0 and on day 5 were analyzed by standard flow cytometry (sd-FCM) with Navios ${ }^{\mathrm{TM}}$ (Beckman Coulter). Statistical analysis was performed with the Wilcoxon test, $\mathrm{p}=0.000^{* *}$.

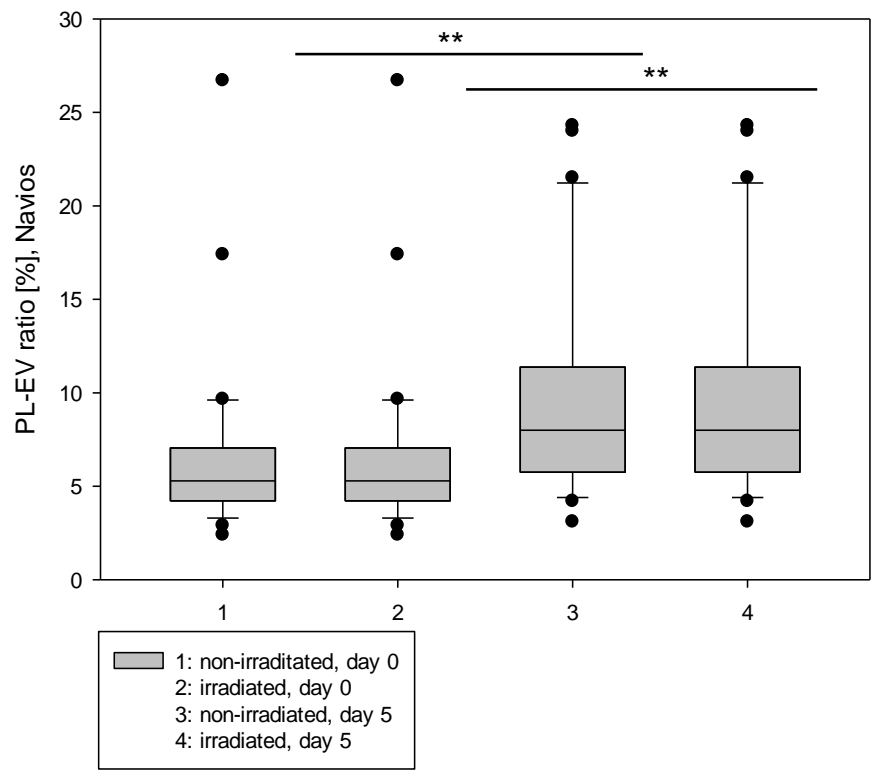

Figure V-26: Box plots of differences in PL-EVs depending on irradiation measured by sd-FCM

First group included 15 non-irradiated PCs (1 and 3) over time; second group included 15 irradiated PCs (2 and 4 ) on day 0 versus day 5 . Analysis was performed by sd-FCM with the Navios $^{\mathrm{TM}}$ (Beckman Coulter). Statistical analysis was performed with the Wilcoxon test and significant differences in both, the first group $\left(p=0.009^{\star *}\right)$ and the second group $\left(p=0.002^{* *}\right)$ are acceptable. 


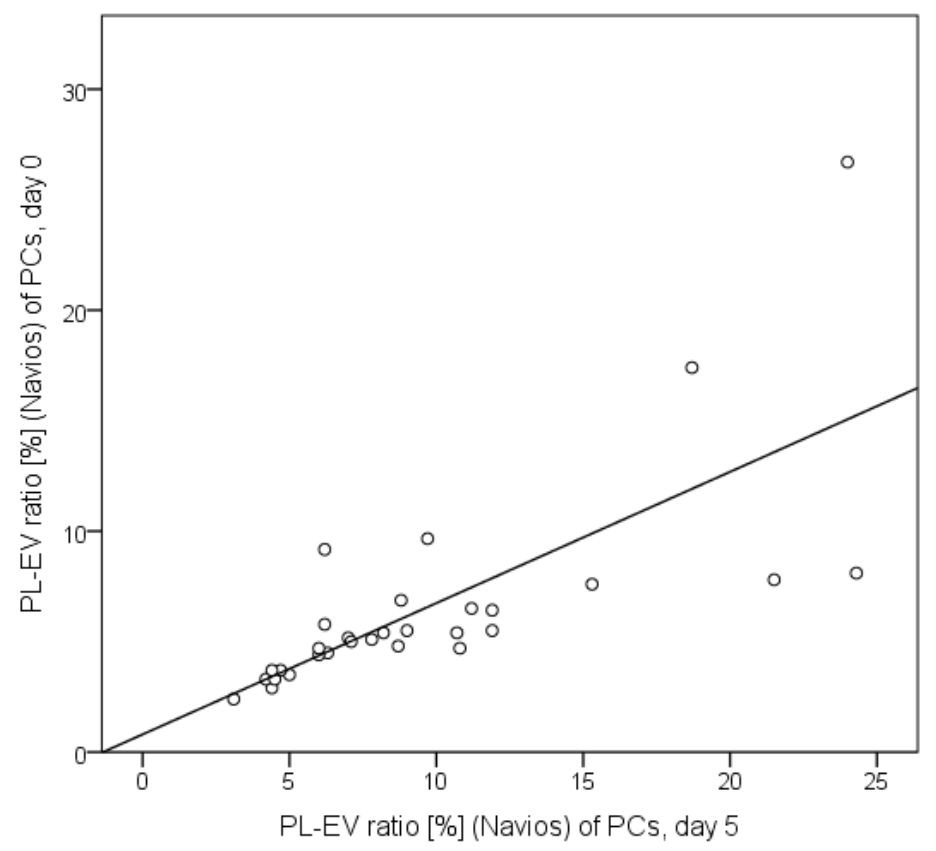

Figure V-27: Scatter plot of the correlation between PL-EVs of fresh versus expired PCs measured by sd-FCM

15 non-irradiated and 15 irradiated PL-EVs, originating from the same PCs $(n=30)$ were analyzed on day 0 and on day 5 and an important correlation between the PL-EV amount on day 0 and day 5 was noticed. Statistical analysis was performed by Pearson's correlation $\left(\mathrm{cC}=0.725^{\star *}, \mathrm{p}=0.000\right)$.

The increase of the PL-EV ratio over time, determined by sd-FCM, was confirmed by the evaluation of PL-EV ratio by hs-FCM, in samples originating from 15 irradiated PCs ( $P=0.006)$, see Figure V-28.

Plasma vesicle concentration did not change in the investigated irradiated PCs over time as measured by NTA (shown in Figure V-29).

Immature platelet fraction, figured in Figure V-30, also grew over time during storage $(p=0.000)$. The values of $\mathrm{pH}$ statistically significantly rose between day 0 [7.2 \pm 0.02 (7.14-7.27)] and day $5[7.35 \pm 0.08(7.0-7.5)]$ in both non-irradiated $(p=0.000)$ and irradiated groups $(p=0.003)$, shown in Figure V-31. 


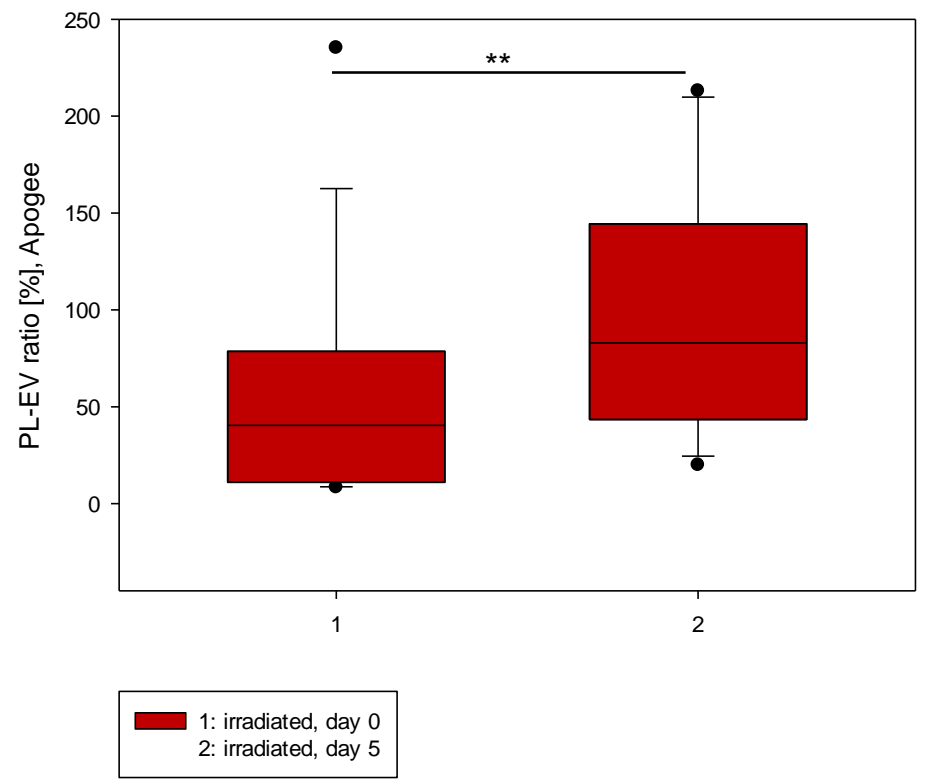

Figure V-28: Box plots of PL-EV ratio of irradiated PCs over time measured by hs-FCM

PL-EVs were analyzed with the Apogee A-50 Micro as CD61-PerCP positive immunolabeled events on day 0 and day 5 in samples of irradiated plateletpheresis concentrates $(n=15)$. Statistically significant difference on day 0 versus day 5 was acceptable $\left(p=0.006^{\star \star}\right.$ with Wilcoxon-test).

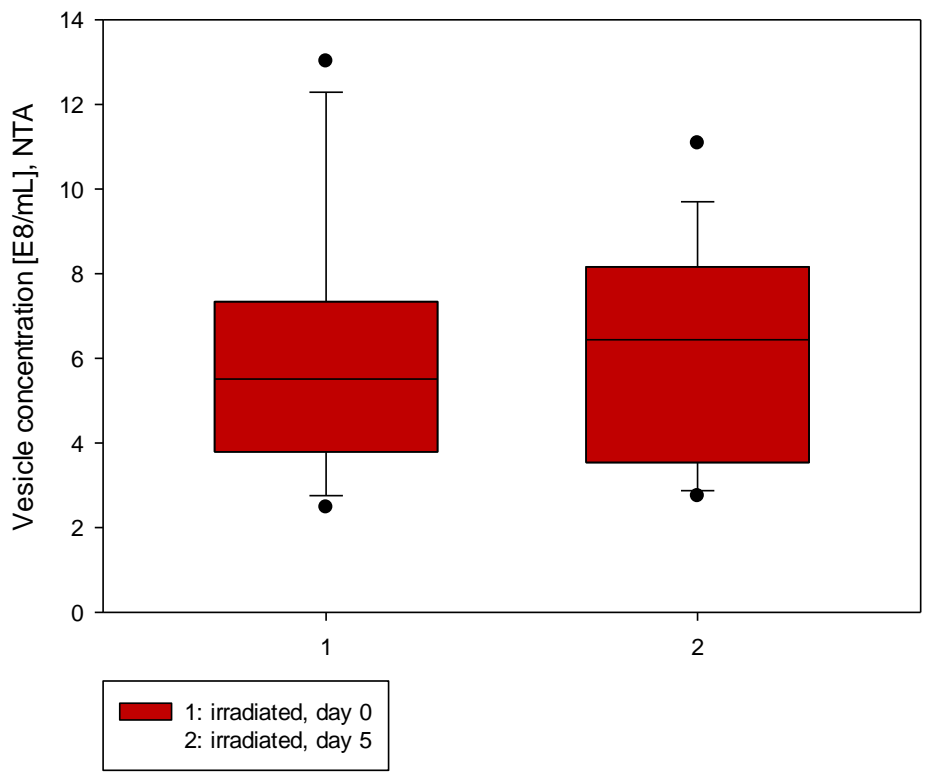

Figure V-29: Box plots of plasma vesicles concentration of irradiated PCs over time measured by NTA

PCs on day 0 and day 5 were analyzed by NTA with NS500 (NanoSight, UK). The irradiated PCs were stored during these days under standard blood banking conditions. No statistically significant difference was observed (Wilcoxon-test, $\mathrm{p}=0.570$ ). 


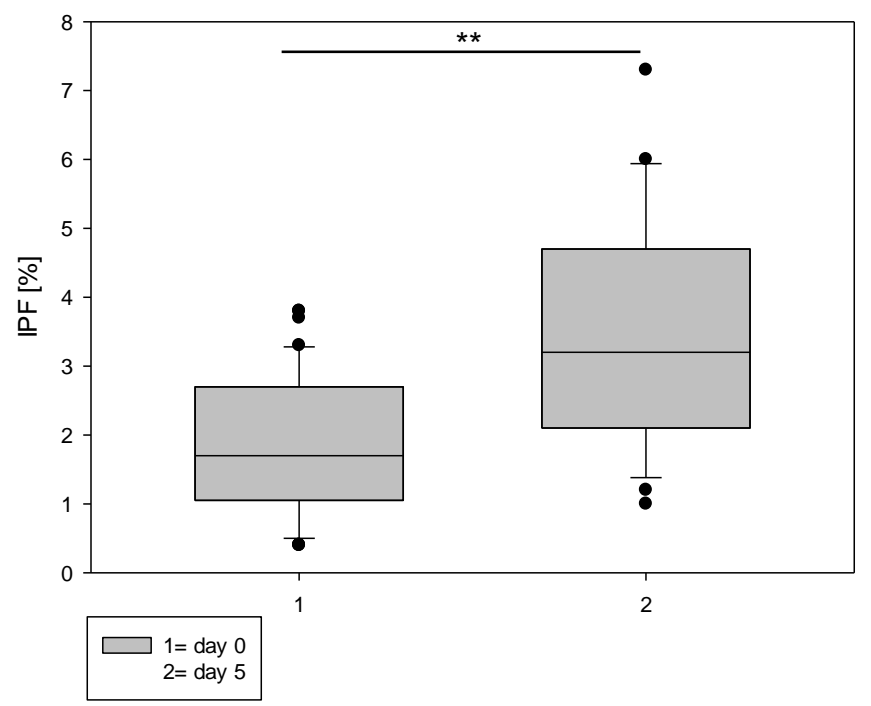

Figure V-30: Box plots of IPF of fresh and stored PCs measured by automated hematology analyzer

Immature platelet fraction (IPF) was measured with XE-1500 (Sysmex) on day $0(n=41)$ and day $5(n=30)$ of PCs (15 irradiated PCs were included). Statistical analysis was performed with the Wilcoxon test, $p=0.000^{\star *}$.

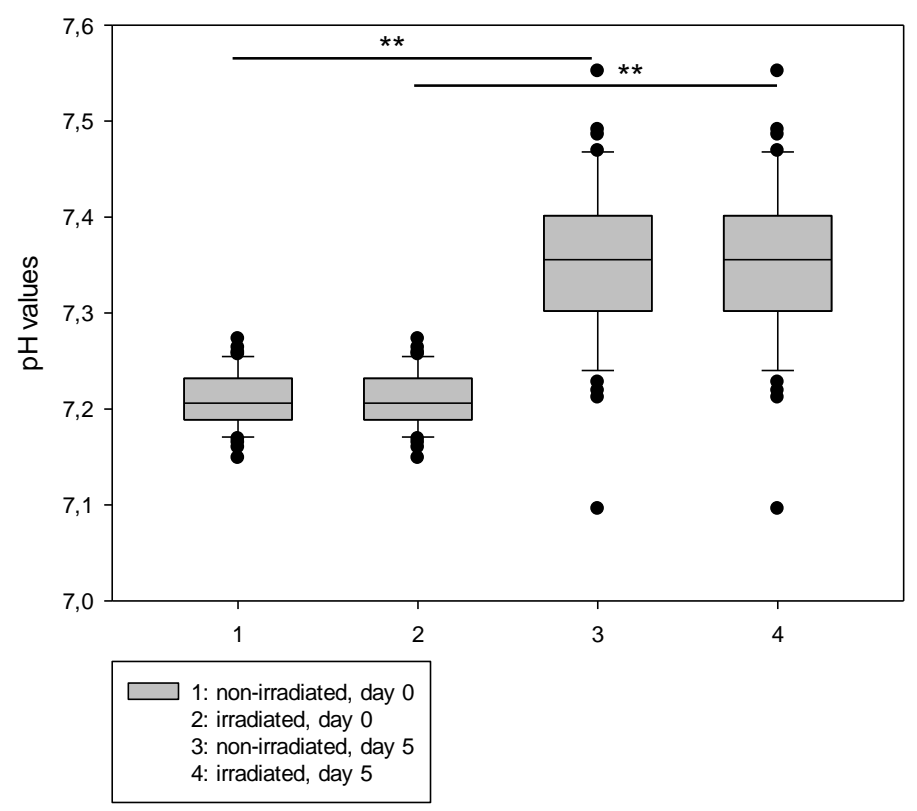

Figure V-31: Box plots of $\mathrm{pH}$ values of PCs over time and the effects of irradiation

PCs were analyzed on day 0 and day $5(n=42 ; 27$ PC were non-irradiated, 15 PC were irradiated) with ABL 90 FLEX (Willich). Statistical analysis was performed with Wilcoxon-test and significant differences within the two groups (non-irradiated, $\mathrm{p}=0.000^{* *}$; irradiated, $\mathrm{p}=0.003^{\star *}$ ) after five-day storage under standard conditions are acceptable. The $\mathrm{pH}$ values play a crucial role in the quality specifications referring to PCs on day 0 and on day 5 . 


\subsubsection{Linear regression analysis of platelet CD62P expression and PL-EV levels (sd- FCM)}

PL-EVs from fresh, non-irradiated PCs significantly negative correlated to the expression of CD62P on platelets $\left(\mathrm{cc}=-0.43^{*}, \mathrm{p}=0.018\right.$, Figure $\mathrm{V}-32$ ). The negative correlation between PL$E V s$ from fresh irradiated PCs and CD62P was not statistically significant $(c c=-0.43, p=0.11)$.

Vice versa, no significant correlation between PL-EVs and CD62P in non-irradiated PCs after expiry was found ( $\mathrm{c}=-0.122, \mathrm{p}=0.545)$, in contrast to irradiated $\mathrm{PCs}$ after expiry $\left(\mathrm{cc}=-0.686^{* *}\right.$, $p=0.005)$.

To explore the dependency of PL-EVs to CD62P, linear regression was used to determine the potential decrease of CD62P caused by vesiculation of fresh platelets. In non-irradiated fresh PCs, one percent of increased PL-EV ratio importantly affected the loss of CD62P expression up to 51.544 percent, $p=0.018$ (with a variability of 18.5 percentage due to the PL-EV ratio from PCs). A negative effect of vesiculation on the p-selectin (CD62P) expression in fresh, nonirradiated platelets from PCs was suggested.

In irradiated PCs, one percent of the increased PL-EV/ PLT ratio affected the loss of CD62P expression by approximately $47.7 \%, \mathrm{p}=0.11$ (with a variability of $18.5 \%$ due to the PL-EV ratio from PCs), but not statistically significant. There was no evidence given for a negative effect of platelet vesiculation on the p-selectin expression in fresh, irradiated platelets in PCs.

By contrast, in expired PCs, the CD62P expression was inversely correlated to PL-EVs in nonirradiated and irradiated PCs. In non-irradiated PCs on day 5, the effect of one percent increase of the PL-EV ratio lowered the CD62P expression by approximately $10.4 \%, p=0.545$ (with a variability of $12.2 \%$ ). However, in irradiated PCs on day 5 for the CD62P expression significant dependency was found with lower levels about $-16.9 \%, p=0.005$ by one percent of a higher PLEV ratio (variability of this effects due to the PL-EVs was found at $47 \%$ ). Thereby, the significantly negative effect of platelet vesiculation on platelet $p$-selectin (CD62P) expression from PCs after expiry was found only in irradiated PCs (shown in Figure V-32). 

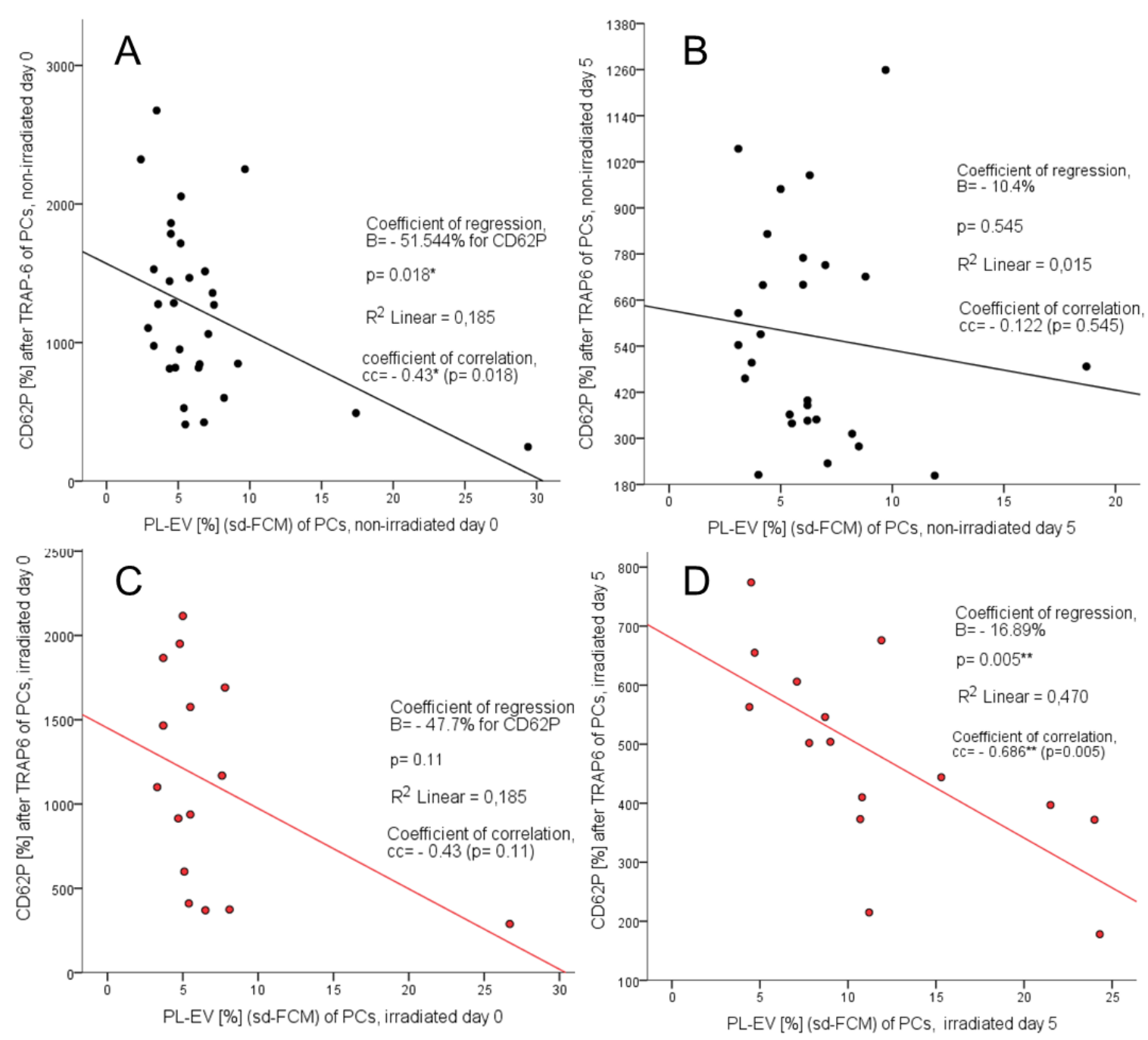

Figure V-32: Scatter plot distribution of PL-EVs in correlation/ linear regression to CD62P externalization

All PC samples were analyzed in order to assess the externalization of $p$-selectin (CD62P-FITC in [\%] of PBS-incubated platelets (as control) after stimulation with TRAP-6 in vitro. In all samples a negative correlation of PL-EV to CD62 expression was found. As figured in scatter plot A-D, a coherence of PL-EV was found with one more unit of PL-EV [\%], that decreased the CD62P externalization of platelets (outlined as coefficient of regression, $B$ in $\%$ ). $R^{2}$, as figured above, is the value of variability of CD62P due to the presence of PL-EVs in the concentrate. Pvalues were estimated using a linear ANOVA model and additionally linear regression was used for CD62P as a dependent variable and PL-EVs as independent variable (T). To correlate both parameters, coefficient of correlation (cc, according to Pearson's correlation) was estimated and significance $(p)$ of coherence was determined. A: For non-irradiated PCs $(n=30)$ on day 0 with observed data (as follows: $B=-51.544, R^{2}=18.5 \%, p=0.018^{*}, T=-2.521, c c=-0.43^{\star}, p=0.018$ ) a significant coherence was assumable. B: In samples drawn from non-irradiated PCs on day 5 $\left(B=-10.4, R^{2}=1.5 \%, p=0.545, T=-0.613\right)$, coefficient of correlation was estimated with $c c=-$ $0.122, p=0.545$ and no significant correlation was found. C: Irradiated fresh PCs $(n=15)$ on day $0\left(B=-47.7, R^{2}=18.5 \%, p=0.11, T=-1.716\right)$ showed no significant relation ( $\left.c c=-0.43, p=0.055\right)$ between PL-EVs and CD62P. D: In samples drawn from irradiated PCs $(n=15)$ on day $5(B=-$ 16.89, $R^{2}=47 \%, p=0.005^{\star *}, T=-3.397$ ) a significant correlation was observed $\left(c c=-0.686^{* *}\right.$, $\mathrm{p}=0.005)$. 


\subsubsection{Correlation between standard PL-EV analysis (sd-FCM) and the new systems (hs- FCM and NTA)}

The aim of the work was to compare a standardized EV analysis to two new methods, i.e. hsFCM (Figure V-34, A) and NTA (Figure V-35 A, B, C).

At first, the methods of PL-EVs analysis, sd-FCM and hs-FCM, applied to fresh non-irradiated PCs were compared to each other ( $\left.\mathrm{cc}=0.72^{* *}, \mathrm{p}=0.002, \mathrm{n}=15\right)$, and the results are displayed in Figure V-33 and Table V-9. Additionally, a positive correlation between sd-FCM and hs-FCM measurements on day 0 with irradiated PCs $(n=15)$, estimated by Pearson, two-way-tail ( $\mathrm{cc}=0.80^{* *}, \mathrm{p}=0.000$ ) was shown.

PL-EVs from irradiated PCs after expiry revealed no statistically significant correlation between both sd-FCM and hs-FCM ( $c c=0.491, p=0.063, n=15)$. Over time, positive correlations were found between PL-EVs of irradiated PCs measured by sd-FCM and the PL-EV concentration for all PL-EVs and, particularly for medium-sized PL-EVs $(180-300 \mathrm{~nm})$ and for the large-sized PLEVs (300-500 nm). No significant correlation between the amount of smallest PL-EV $(<180 \mathrm{~nm}$, measured by hs-FCM) and the amount of large PL-EV (>500 nm, measured by sd-FCM) populations were found. Both groups of PL-EVs, the medium and the large, from fresh nonirradiated PCs, showed a significant positive correlation.

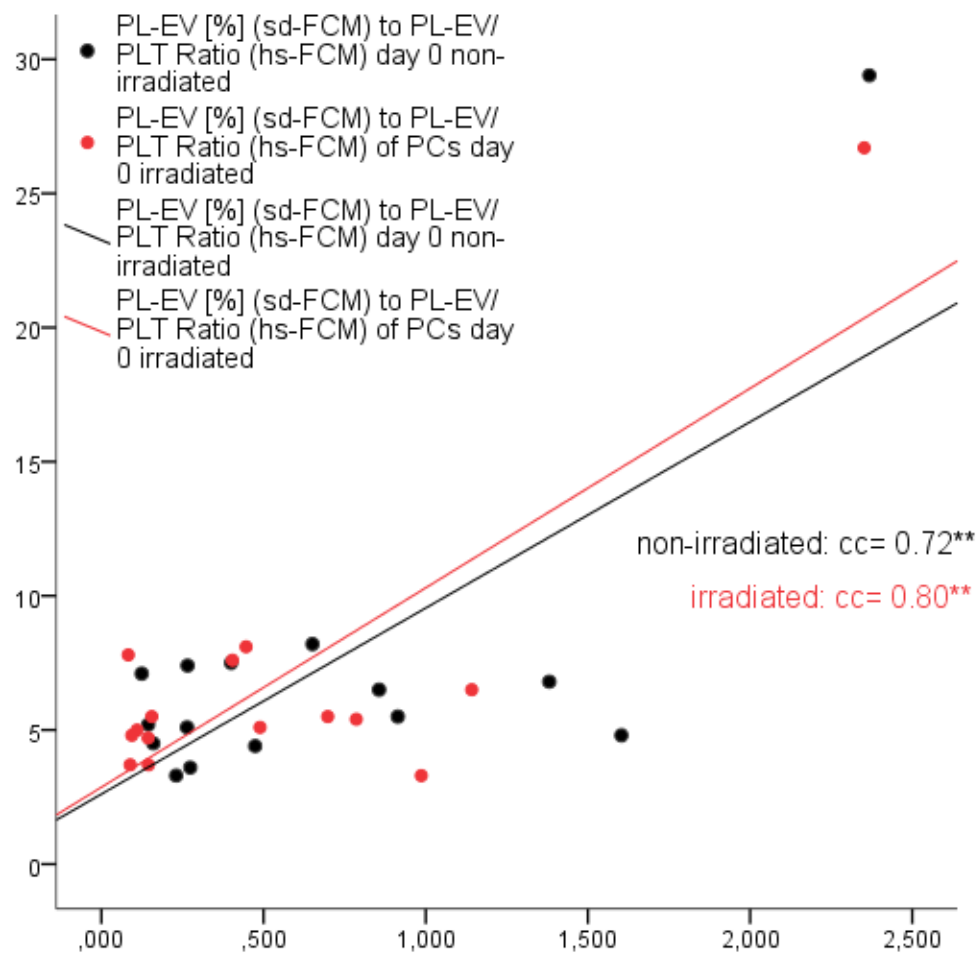

Figure V-33: Comparison of sd-FCM and hs-FCM PL-EV analysis on day 0

Pearson's correlation indicated a positive correlation between sd-FCM ( $y$-axis) and hs-FCM ( $x$ axis) for both non-irradiated PCs on day $0\left(\mathrm{cC}=0.72^{* *}, \mathrm{p}=0.002, \mathrm{n}=15\right)$ and irradiated PCs on day $0\left(\mathrm{cc}=0.8^{* *}, \mathrm{p}=0.000, \mathrm{n}=15\right)$ with statistical significance. 
Secondly, the concentration of plasma vesicles, measured by NTA, from fresh non-irradiated PCs ( $c c=0.014, p=0.962, n=15$ with Pearson analysis) and fresh irradiated PCs ( $c c=-0.068$, $\mathrm{p}=0.811, \mathrm{n}=15$ with Pearson analysis) were correlated to PL-EVs analyzed by sd-FCM (not figured). No statistically significant correlation was found. After expiry (data not shown), PL-EVs from irradiated PCs evaluated by sd-FCM did not significantly correlate to the concentration of plasma vesicles evaluated by NTA ( $c c=0.228, p=0.415, n=15$ by Pearson's correlation).
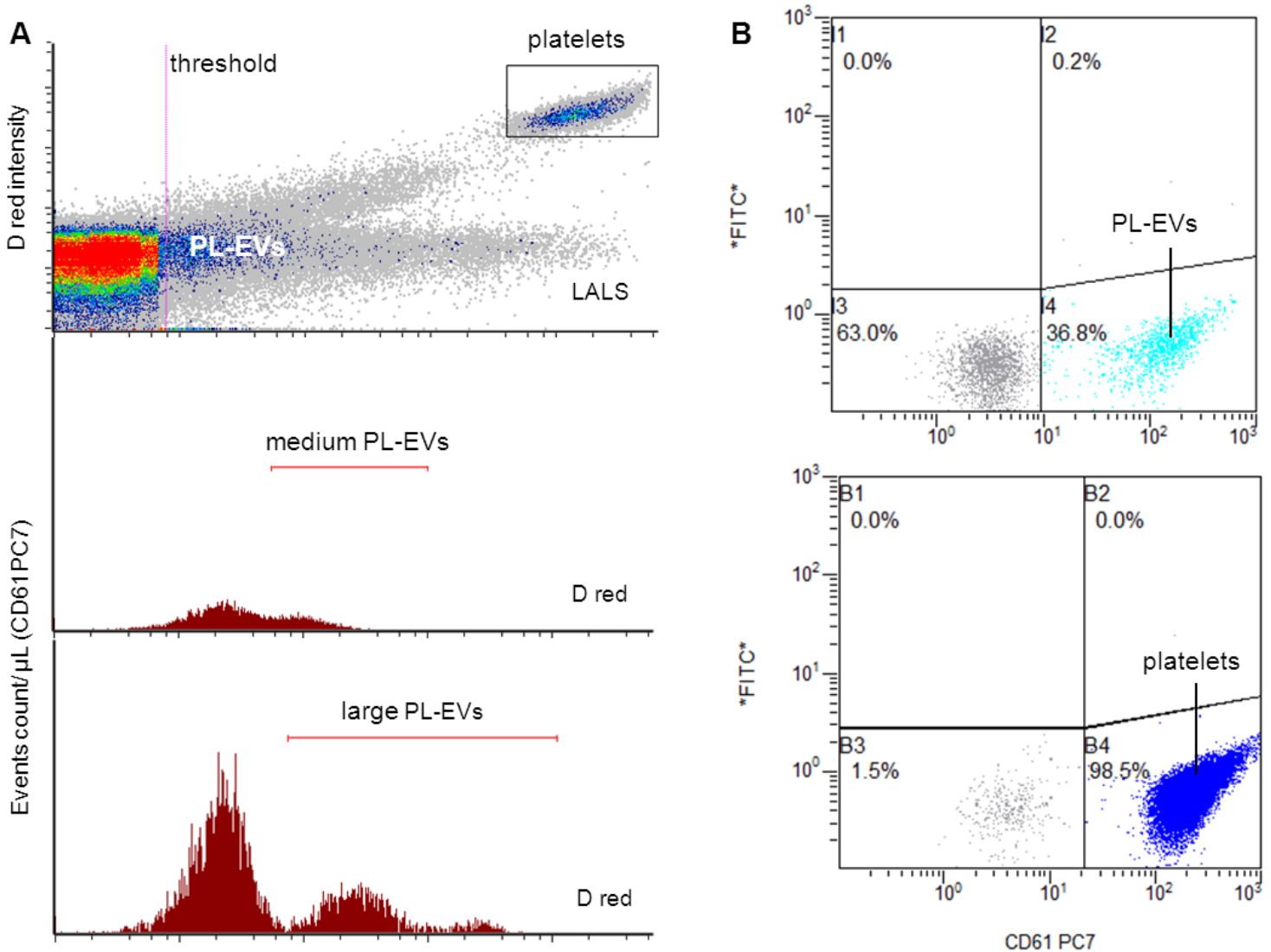

Figure V-34: PL-EV analysis by hs-FCM (A) and sd-FCM (B)

A: PL-EVs representative for fresh, non-irradiated PCs were determined by high sensitive flow cytometry (hs-FCM) [CD61-positive events/ $\mu \mathrm{L}=1037, \mathrm{PL}-\mathrm{EV}$ ratio=47\%]. B: Analysis representative for fresh, non-irradiated PCs was carried out by standard flow cytometry (sdFCM) [CD61-positive events $/ 500 \mu \mathrm{L}$ diluted sample=1050, PL-EV ratio=4.8\%]. 


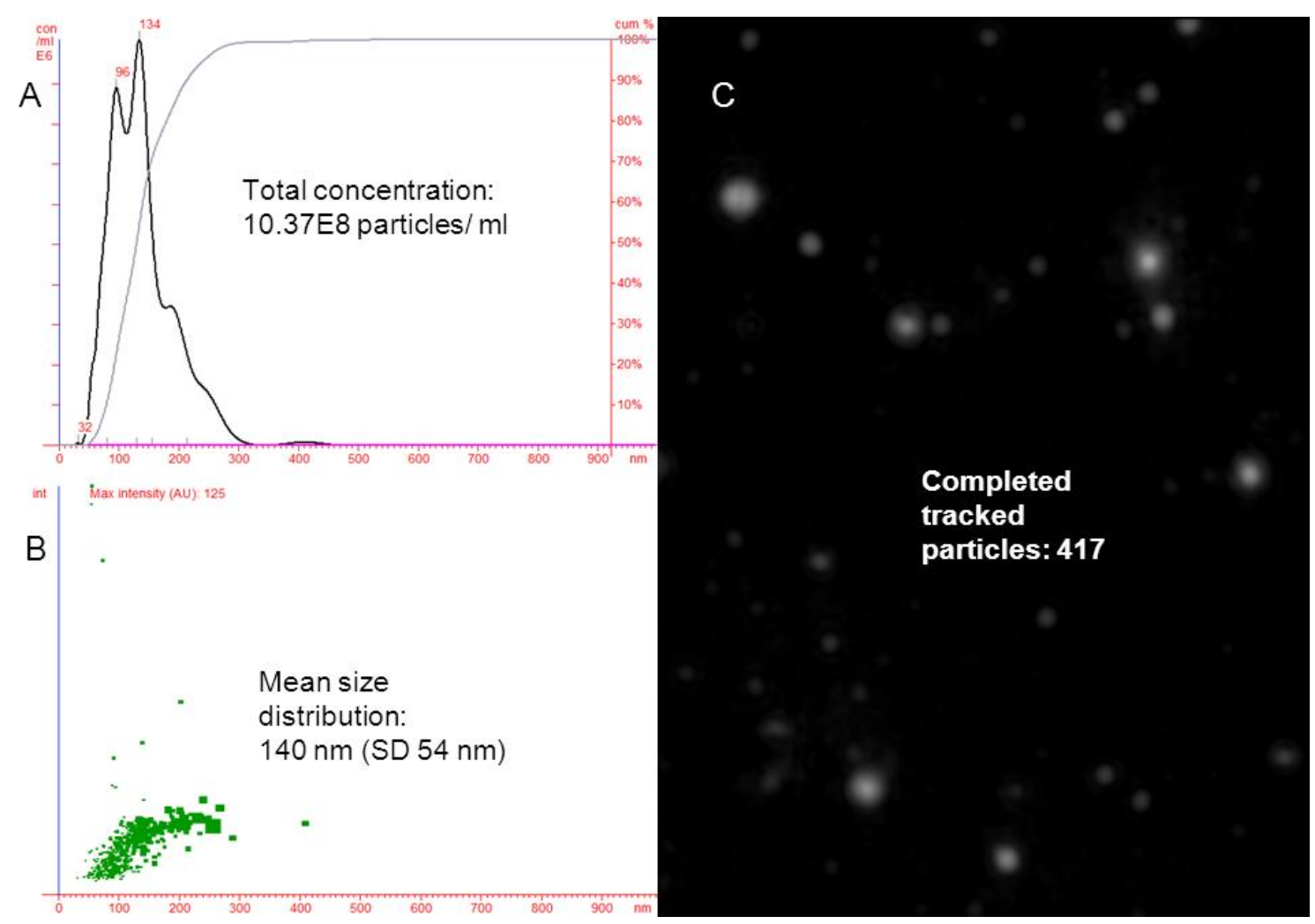

Figure V-35: Evaluation of plasma vesicles from PCs by NTA

A: Black Graph represents concentration $\left(\times 10^{8} / \mathrm{mL}\right)$ of plasma vesicles representative for fresh, non-irradiated PC in correlation to vesicle size $(\mathrm{nm})$. B: Size of vesicles versus intensity of scattered light is shown and green boxes represent every single vesicle measured. C: Freeze image of a video representative for tracked vesicles (white dots) is shown. 
Table V-9: PL-EV analysis by sd-FCM versus hs-FCM or NTA

\begin{tabular}{|c|c|c|c|c|c|}
\hline & & & \multicolumn{3}{|c|}{ PL-EV [\%] (sd-FCM) } \\
\hline & & & $\begin{array}{c}\text { day } 0 \\
\text { non-irradiated }\end{array}$ & $\begin{array}{c}\text { day } 0 \\
\text { irradiated }\end{array}$ & $\begin{array}{c}\text { day } 5 \\
\text { irradiated }\end{array}$ \\
\hline \multirow{18}{*}{$\sum_{\substack{5 \\
\dot{1}}}$} & \multirow{3}{*}{$\begin{array}{c}\text { Small } \\
\text { PL-EVs } \\
{[\text { Events/ } \mu \mathrm{L}]}\end{array}$} & \multirow{3}{*}{$\begin{array}{l}\text { day } 0, \text { non-irradiated } \\
\text { day } 0, \text { irradiated } \\
\text { day } 5, \text { irradiated }\end{array}$} & 0.493 & & \\
\hline & & & & -0.025 & \\
\hline & & & & & 0.474 \\
\hline & \multirow{3}{*}{$\begin{array}{c}\text { Medium } \\
\text { PL-EVs } \\
{[\text { Events/ } \mu \mathrm{L}]}\end{array}$} & \multirow{3}{*}{$\begin{array}{l}\text { day } 0, \text { non-irradiated } \\
\text { day } 0, \text { irradiated } \\
\text { day } 5, \text { irradiated }\end{array}$} & $0.753^{n \pi}$ & & \\
\hline & & & & $0.801^{* *}$ & \\
\hline & & & & & 0.479 \\
\hline & \multirow{3}{*}{$\begin{array}{c}\text { Large } \\
\text { PL-EVs } \\
{[\text { Events } / \mu \mathrm{L}]}\end{array}$} & \multirow{3}{*}{$\begin{array}{l}\text { day } 0, \text { non-irradiated } \\
\text { day } 0 \text {, irradiated } \\
\text { day } 5, \text { irradiated }\end{array}$} & $0.897^{n n}$ & & \\
\hline & & & & $0.902^{\star \star}$ & \\
\hline & & & & & $0.864^{\star \star}$ \\
\hline & \multirow{3}{*}{$\begin{array}{l}\text { PLT count } \\
{[\text { Events/ } \mu \mathrm{L}]}\end{array}$} & \multirow{3}{*}{$\begin{array}{l}\text { day } 0, \text { non-irradiated } \\
\text { day } 0 \text {, irradiated } \\
\text { day } 5 \text {, irradiated }\end{array}$} & 0.243 & & \\
\hline & & & & 0.025 & \\
\hline & & & & & 0.467 \\
\hline & \multirow{3}{*}{$\begin{array}{c}\text { All } \\
\text { PL-EVs } \\
{[\text { Events } / \mu L]}\end{array}$} & \multirow{3}{*}{$\begin{array}{l}\text { day } 0, \text { non-irradiated } \\
\text { day } 0, \text { irradiated } \\
\text { day } 5 \text {, irradiated }\end{array}$} & $0.824^{n n}$ & & \\
\hline & & & & $0.848^{\star \star}$ & \\
\hline & & & & & $0.671^{\star *}$ \\
\hline & \multirow{3}{*}{$\begin{array}{c}\text { PL-EV Ratio } \\
{[\%]}\end{array}$} & \multirow{3}{*}{$\begin{array}{l}\text { day } 0, \text { non-irradiated } \\
\text { day } 0 \text {, irradiated } \\
\text { day } 5 \text {, irradiated }\end{array}$} & $0.72^{\star \star}$ & & \\
\hline & & & & $0.8^{\star *}$ & \\
\hline & & & & & 0.491 \\
\hline \multirow{6}{*}{ 乒 } & \multirow{3}{*}{$\begin{array}{c}\text { Plasma EV } \\
\text { concentration } \\
{\left[\times 10^{8} / \mathrm{mL}\right]}\end{array}$} & \multirow{3}{*}{$\begin{array}{l}\text { day } 0, \text { non-irradiated } \\
\text { day } 0, \text { irradiated } \\
\text { day } 5, \text { irradiated }\end{array}$} & 0.014 & & \\
\hline & & & & -0.068 & \\
\hline & & & & & 0.109 \\
\hline & \multirow{3}{*}{$\begin{array}{c}\text { Size } \\
\text { distribution } \\
{[\mathrm{nm}]}\end{array}$} & \multirow{3}{*}{$\begin{array}{l}\text { day } 0, \text { non-irradiated } \\
\text { day } 0, \text { irradiated } \\
\text { day } 5 \text {, irradiated }\end{array}$} & -0.139 & & \\
\hline & & & & 0.034 & \\
\hline & & & & & 0.228 \\
\hline
\end{tabular}

Correlation is assumed as significant at $p \leq 0.01^{* *}$ and $p \leq 0.05^{*}$ with two-tailed Pearson analysis. (hs-FCM denotes high sensitivity flow cytometry; sd-FCM denotes standard flow cytometry).

\subsubsection{Correlation between platelets CD62P and PL-EV analyses by hs-FCM and NTA}

Although a significant correlation between hs-FM and sd-FM analyses of PL-EVs has been found, it was also investigated whether there is a relation between PL-EV ratio determined by hs-FM and platelet p-selectin expression upon TRAP-6 stimulation. A significant negative correlation was found among all measured samples from non-irradiated and irradiated PCs on day 0 and from irradiated PCs on day $5\left(\mathrm{cc}=-0.736^{* *}(\mathrm{p}=0.002) ; \mathrm{cc}=-0,646^{\star *} \quad(p=0,005)\right.$; $\mathrm{cc}=-0.660^{* *}(\mathrm{p}=0.007)$ respectively). A strong negative correlation is shown in Figure $\mathrm{V}-36$ and data are presented in Table $\mathrm{V}-10$. 


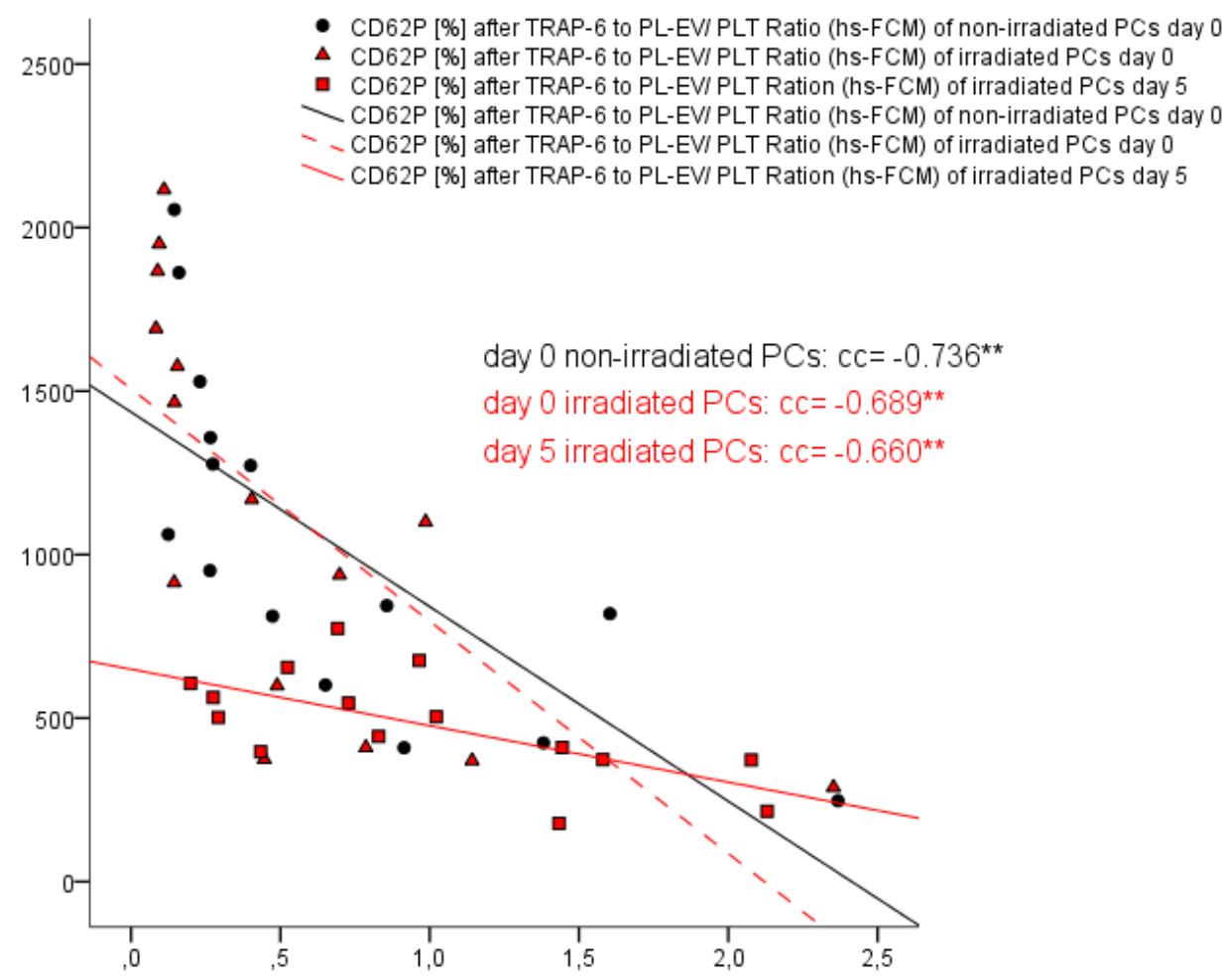

Figure V-36: Correlation between CD62P expression and the PL-EV-ratio by hs-FCM

Non-irradiated PCs and irradiated PCs on day 0 and day 5 were analyzed $(n=15)$. A strong negative relation between the PL-EV ratio ( $x$-axis) and activatability of platelets ( $y$-axis) was found (determined with the correlation coefficient, two-way-tailed). Values of CD61-positive PLEV events were correlated to CD61-positive platelets events and graphically presented as dots. Subsequently, the ratio of PL-EVs to platelets was calculated. Values of CD62P expression are shown as the mean in percent to basal CD62P expression. Correlation was assumed as significant at $p \leq 0.01^{* *}$ and $p \leq 0.05^{*}$.

A statistically significant correlation between the plasma vesicle concentration measured by NTA and platelet CD62P expressed after TRAP-6 stimulation was not detectable. Neither in 15 fresh non-irradiated ( $\mathrm{cc}=0.264, \mathrm{p}=0.343)$ / irradiated $\mathrm{PCs}(\mathrm{cc}=0.351, \mathrm{p}=0.2)$, or 15 irradiated expired PCs ( $c c=0.118, p=0.676)$ any statistically significant correlation between vesicles and CD62P expression has been found (not figured, analyzed by Pearson's test of correlation coefficient, one-way-tailed). Similar results showing no important correlation between CD62P after TRAP-6 stimulation and the size of plasma vesicles, measured by NTA, were obtained (data reported in Table V-10). 
Table V-10: Correlation between platelet CD62P expression after TRAP-6 determined by hsFCM and by NTA

Correlation is assumed as significant at $\mathrm{p} \leq 0.01^{* *}$ and $\mathrm{p} \leq 0.05^{*}$ (two-tailed by Pearson' correlation).

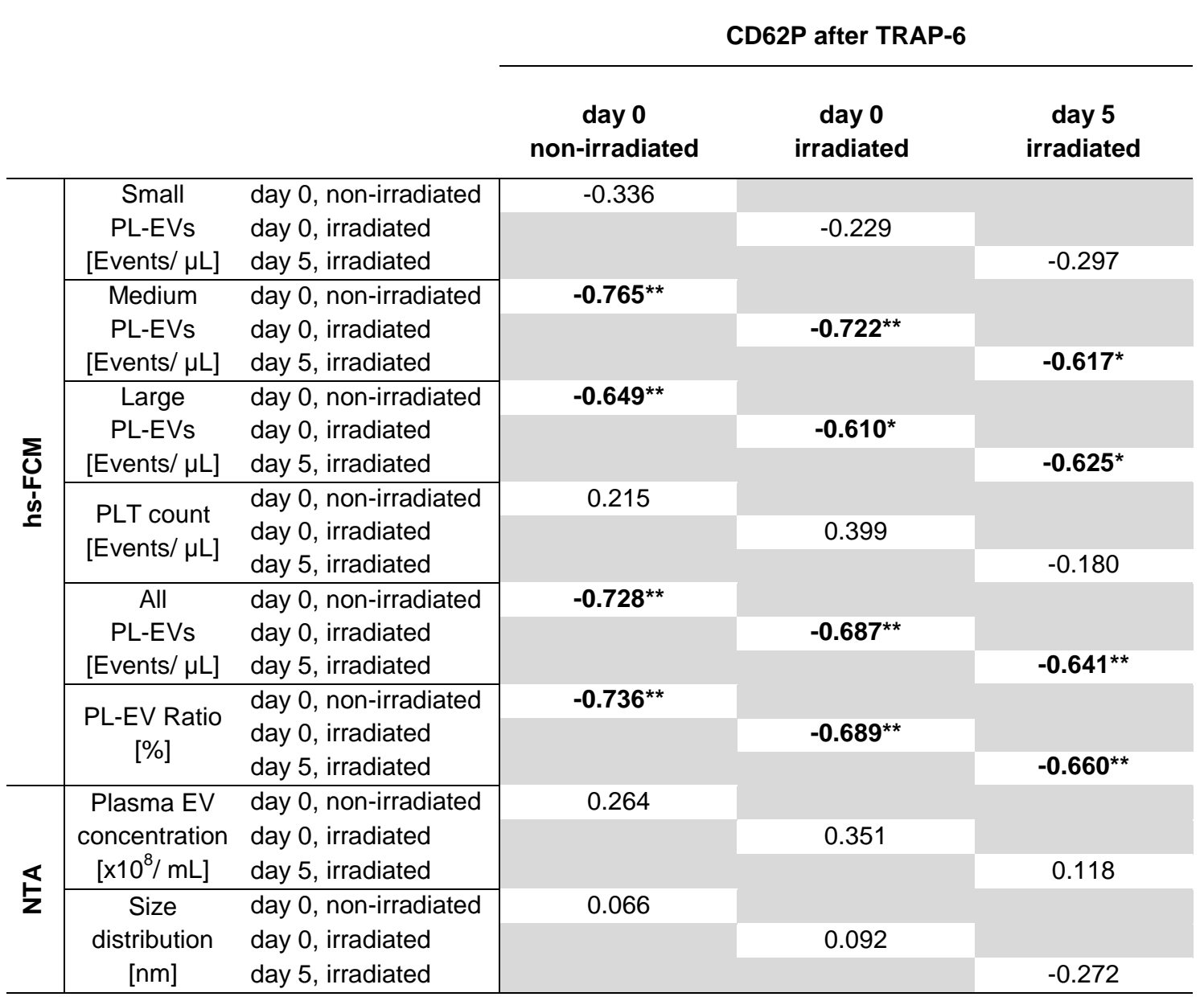

The method of NTA turned out to be unhelpful in answering the main question whether hs-FCM or NTA are useful as a quality control approach. However, with hs-FCM it was possible to show additional significant correlation between the CD62P expression after TRAP-6 stimulation and single subpopulations of PL-EVs and between the CD62P expression and all PL-EVs. Not only was the PL-EV ratio visibly negative in correlation to the responsiveness of platelets, but the concentration of PL-EVs was recorded as CD61-positive events per $\mu \mathrm{L}$.

The population of all PL-EVs included PL-EVs smaller than $180 \mathrm{~nm}$, medium-sized PL-EV with a size range of $180-300 \mathrm{~nm}$ and large PL-EVs with diameters ranging from 300 to $500 \mathrm{~nm}$. The smallest PL-EVs as a minor part of the whole fraction negatively correlated to platelet CD62P expression, but did not reach the level of significance. In contrast, the medium and large PLEVs showed significance for negative correlation to platelet CD62P expression after TRAP-6 
and to the PL-EV ratio analyzed by hs-FCM. Correlation between values of PL-EVs, especially of the medium-sized PL-EVs, and the expression of CD62P was more significant than the correlation between semiquantitative analysis of PL-EVs and CD62P analysis (for both nonirradiated and irradiated PCs).

To visualize this correlation, three dot plots were created for $15 \mathrm{PCs}$, analyzed on day 0 (shown in Figure V-37, then investigated upon irradiation (Figure V-38) and finally after five-day storage (Figure V-39).

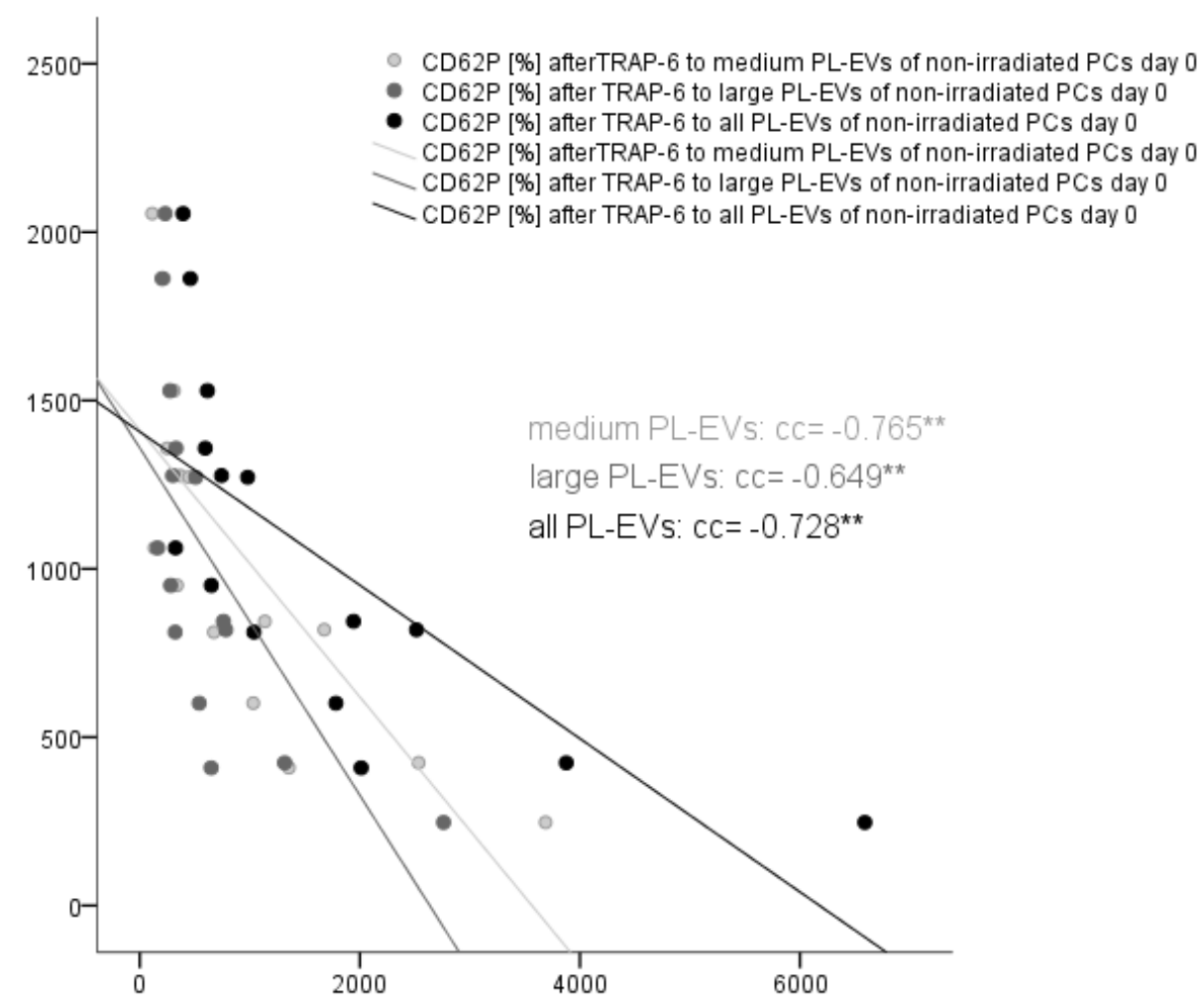

Figure V-37: Correlation between CD62P (after stimulation with TRAP-6) and PL-EV subpopulations in fresh non-irradiated PCs measured by hs-FCM

Non-irradiated PCs on day $0(n=15)$ were analyzed. A strong negative relation between PL-EVs (x-axis) and activatability of platelets (y-axis) was found, determined with the correlation coefficient (two-way-tailed). Levels of the CD61-positive PL-EVs are pictured as the mean [Events/ $\mu \mathrm{L}$ ], values of CD62P expression are shown as the mean in percent to basal CD62P expression. Correlation was assumed as significant at $p \leq 0.01^{* *}$ and $p \leq 0.05^{*}$. 


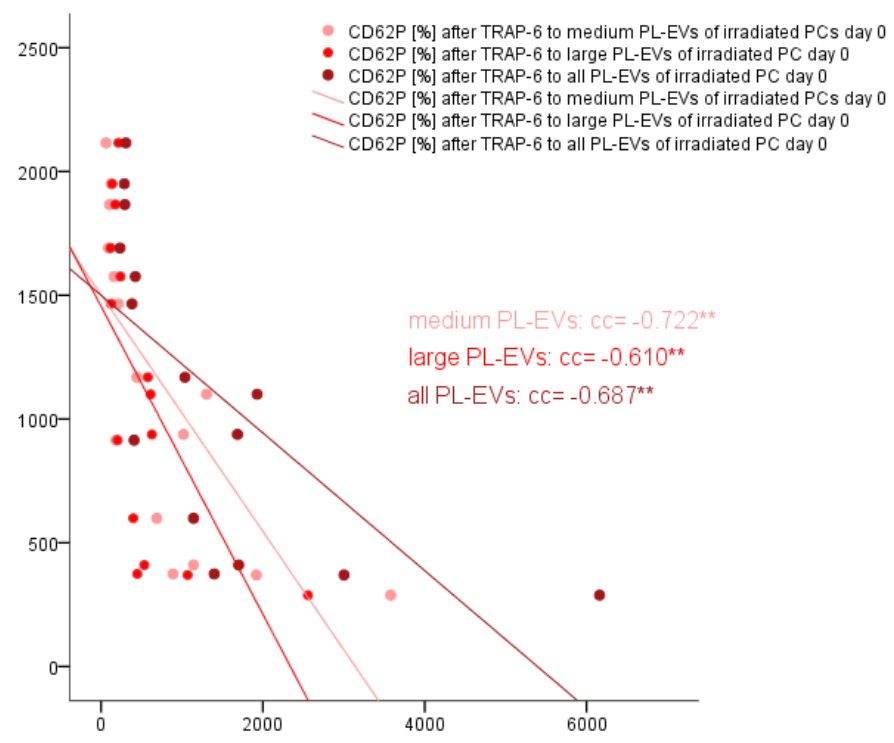

Figure V-38: Correlation between CD62P (after TRAP-6 stimulation) and PL-EV subpopulations in fresh irradiated PCs measured by hs-FCM

Irradiated PCs on day $0(n=15)$ were analyzed. A strong negative relation between PL-EVs $(x-$ axis) and activatability of platelets (y-axis) was found, analyzed with the correlation coefficient (two-way-tailed). Levels of the CD61-positive PL-EVs are pictured as the mean [Events/ $\mu \mathrm{L}$ ], values of CD62P expression are shown as the mean in percent to basal CD62P expression. Correlation was assumed as significant at $p \leq 0.01^{* *}$ and $p \leq 0.05^{*}$.

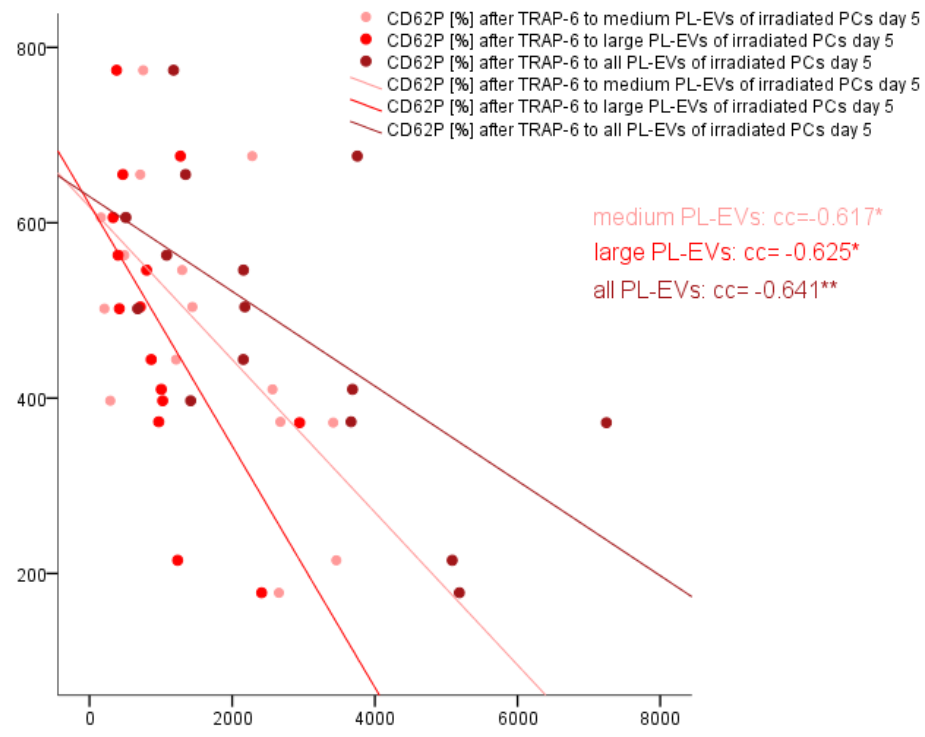

Figure V-39: Correlation between CD62P (after stimulation with TRAP-6) and PL-EV subpopulations in senescent irradiated PCs measured by hs-FCM

Irradiated PCs on day $5(n=15)$ were analyzed. A strong negative relation between PL-EVs $(x-$ axis) and activatability of platelets (y-axis) was found, analyzed with the correlation coefficient (two-way-tailed). Levels of the CD61-positive PL-EVs are pictured as the mean [Events/ $\mu \mathrm{L}$ ], the values of CD62P expression are shown as the mean in percent to basal CD62P expression. Correlation was assumed as significant at $p \leq 0.01^{* *}$ and $p \leq 0.05^{*}$. 
In contrast to the sd-FCM analysis, the hs-FCM measured quantitative PL-EV levels. The correlation between platelet CD62P expression and absolute PL-EV values analyzed by hsFCM was more significant, suggesting that there is a relevant relation of vesiculation and platelet function in PCs at any time. A negative correlation between CD62P expression and PLEVs in samples of fresh non-irradiated PCs, fresh irradiated PCs as well as of irradiated PCs after expiry was observed. To use PL-EVs as a parameter in a quality control of PCs, the new system, i.e. hs-FCM method, is favored over sd-FCM in PL-EV analysis. With hs-FCM, PL-EVs analysis provides a plausibility check which enables estimating platelet functionality.

\subsection{Extracellular vesicle composition in plateletpheresis concentrates and red blood cell units}

Due to shear stress during plateletpheresis, artificial extracellular vesicle released from other blood cells such as red blood cells (RBCs) or leukocytes might appear and vesicles may be stored in PCs. Therefore, we tested, whether PCs contain cell-derived EVs originating from red blood cells (RBC-EVs) or leukocytes (L-EVs).The analysis was performed under the same conditions as described for PL-EVs. In PCs, independent of the time of analysis (day 0, day2 and day 5), the ratio of RBC-EVs in PCs as measured by sd-FCM was less than $2 \%$ and L-EVs were not measurable, shown in Figure V-40. PL-EV analysis by hs-FCM showed similar results, that means, no relevant ratios of RBC-EVs were found (data not shown). The composition of cell-derived vesicles was constituted mostly of CD61-positive PL-EVs (98-99\%) of all measured events. In flow cytometric analysis, vesicles were labeled with moABs against CD45 as a panleukocyte marker, CD61 as a platelet marker as well as against CD235 as a marker for RBCs. 
A

All PCs ( $n=30)$

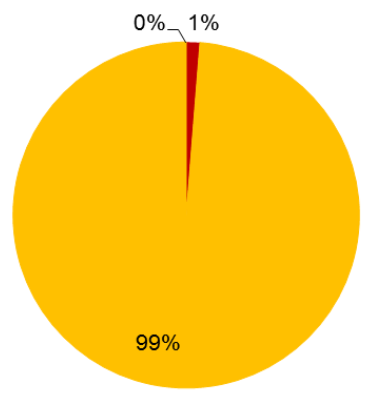

C

PCs on day $2(n=2)$

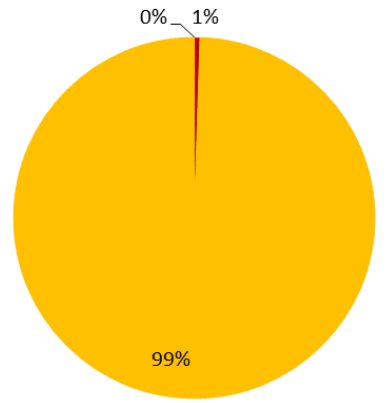

E

PCs day 0,2 and day $5(n=15) 1: 100$

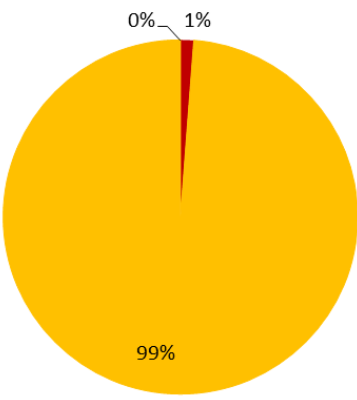

B PCs on day $0(n=23)$

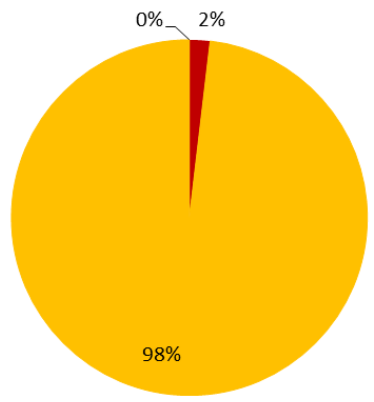

D PCs on day $5(n=5)$

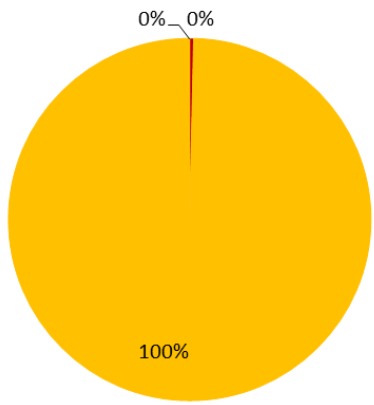

F PCs day 0 and day $5(n=15) 1: 500$

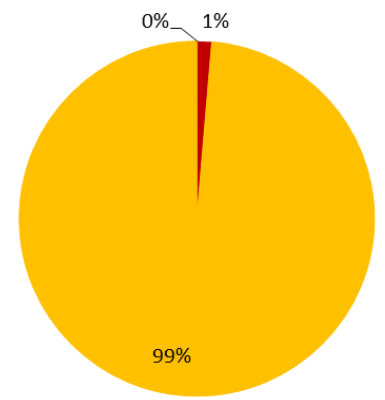

Figure V-40: Composition of cell-derived vesicles in PCs

Vesicles of a size of approx. 300 - $500 \mathrm{~nm}$ were labeled with monospecific fluorescent antibodies against erythrocytes (red), platelets (yellow) and leukocytes (green, no events measurable) and analyzed by sd-FCM (Navios ${ }^{\mathrm{TM}}$ ). The main fraction of vesicles in PCs originated from platelets, whereas vesicles from leukocytes were not measurable (A-F). Vesicles from erythrocytes represented a ratio less than $2 \%$ on day 0 (B), on day 2 (C) and on day 5 (D). For samples in different dilutions, one of 1:100 (E) and of 1:500 (F) no significant changes in composition were found.

According to the method of preparation of platelet-derived exosomes (PL-EXs) from PCs, RBCderived EVs were prepared by ultra-centrifugation from RBC units. Different pellets were obtained and analyzed. The pellet after four centrifugation steps with $120.000 \mathrm{~g}$ was named P4 (see Figure V-41, B). The cell-derived vesicles (P4) of RBC units did not originate from the main cell component - the RBCs. In contrast, undiluted samples of RBC units comprise of RBC-EVs amounting to $80 \%$ of all cell-derived vesicles (see Figure V-41, A). Values of RBC-EVs, PL-EVs 
and L-EVs from the EV-pellets (P4), which included exosomes more concentrated than the supernatant of RBC units, let assume, that, especially in the third fraction after density gradient centrifugation (Figure V-41, C-E), only PL-EVs were obtained.
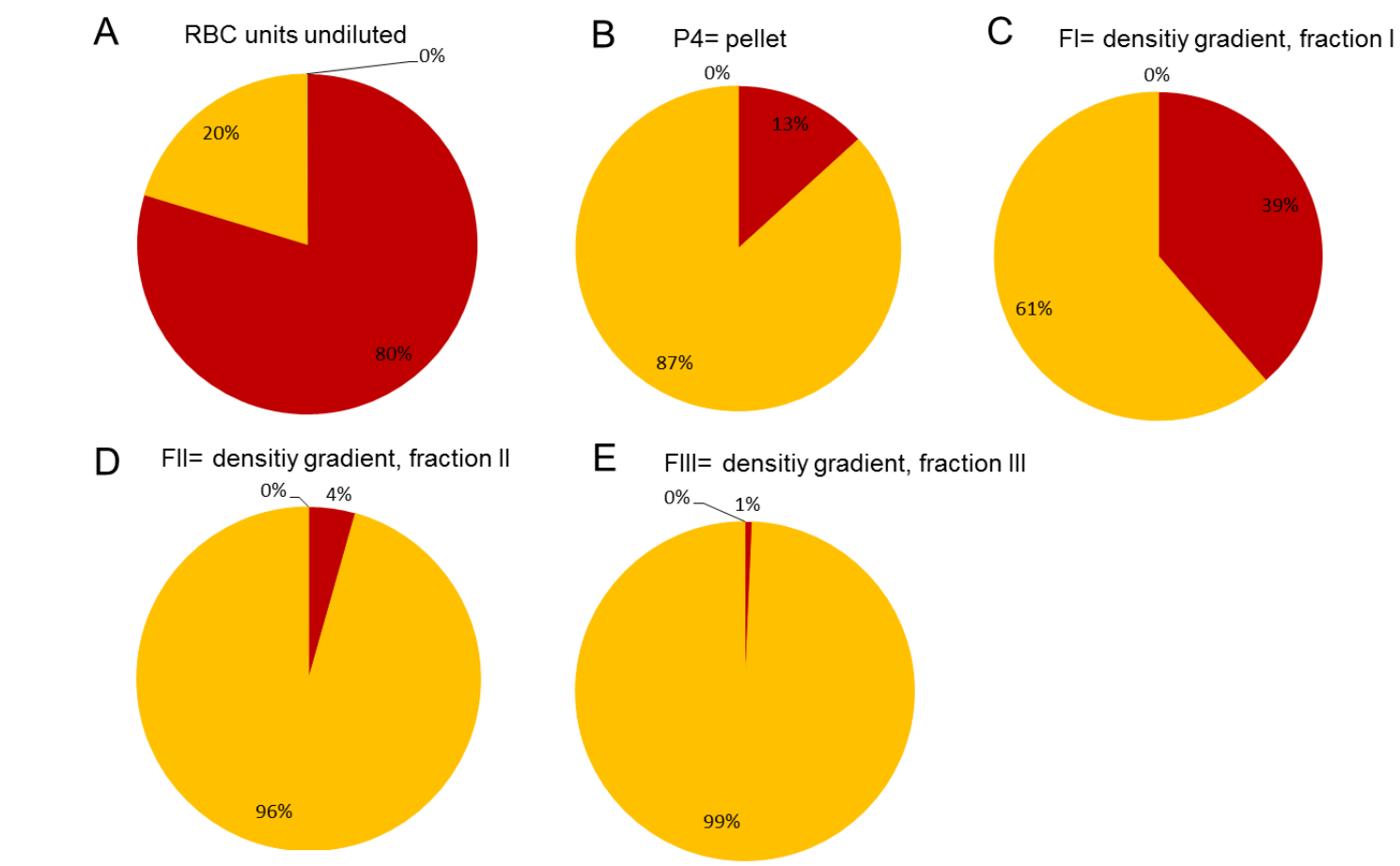

Figure V-41: Pie chart of the cell-derived EV percentage in red blood cell units

EV analysis of RBC units ( $n=3$ ) was performed by sd-FCM with Navios ${ }^{\mathrm{TM}}$. The main fraction of undiluted concentrates $(A)$ of vesicles originated from erythrocytes, whereas the vesicle pellet $(\mathrm{P} 4, \mathrm{~B})$ in a tenth part of one unit included predominantly platelet-derived vesicles. These results were confirmed by EV-analysis from single fractions of this pellet, i.e. the fraction I (C), fraction II (D) and fraction III (E) collected after density gradient centrifugation. Vesicles from leukocytes were not found.

In earlier centrifugation steps during preparation of pellet 4 (P4), as described in Table IV-2, three pellets were obtained after wash steps (90 min, $120.000 \mathrm{~g}$ ) and they were quantitatively analyzed by hs-FCM.

Exemplarily, the PL-EV event count per $\mu \mathrm{L}$ was measured in one quarter of pellet 3 comprising of vesicles of a size ranging from 30 to $500 \mathrm{~nm}$ (Figure V-42). Interestingly, in this vesicle pellet of RBC units, PL-EV concentration was found at the level of $2056^{*} / \mu \mathrm{L}$. However, the RBC-EV concentration from the same pellet was determined at the level of $967^{*} / \mu \mathrm{L}$ (* shown in Figure $\mathrm{V}-42$ ). With the help of the semiquantitative EV analysis performed by sd-FCM, it was possible to demonstrated that in $500 \mu \mathrm{L}$ of pellet 3 there were 13538 CD61-positive events (PL-EVs) and 20070 CD235a-positive events (RBC-EVs) (data not shown). 


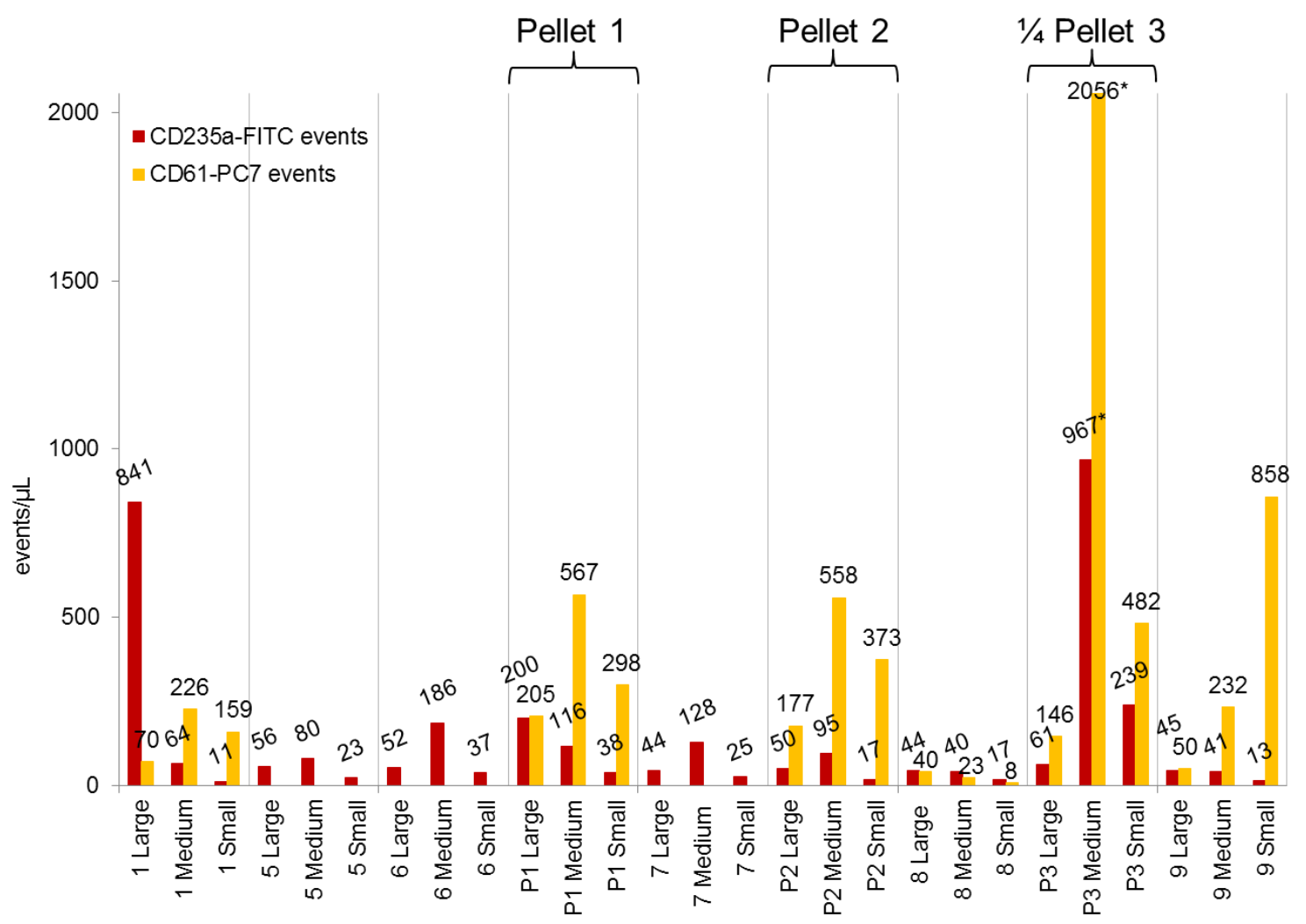

Figure V-42: Platelet-derived EVs and erythrocyte-derived EVs from red blood cell units

EV analysis of red blood cell units $(n=2)$ was performed by hs-FCM with A50-Micro Flow Cytometer (Apogee Flow Systems Ltd., UK). For each single preparation step (1, 5 - 9, see Table IV-2) and pellets 1 - 3 (P1 - P3), vesicle quantification was reported for small $(\leq 180 \mathrm{~nm})$, medium $(180-300 \mathrm{~nm})$ and large $(\geq 300 \mathrm{~nm})$ vesicles. Values are reported as mean and written in italic for CD235a-positive labeled vesicles or normal written for CD61-positive labeled vesicles. Marked values $\left({ }^{*}\right)$, exemplarily, represent unequal distribution between PL-EVs and RBC-EVs in a pellet of a RBC unit.

A size diagram of all cell-derived and plasma containing vesicles stemming from RBC units and a diagram of vesicle concentration are depicted in a form of line charts representing one single pellet fraction (see Figure V-43).

Particle size in the first pellet (Pellet 1, Figure V-43) was determined with median particle size of $228 \mathrm{~nm}$, to the second pellet (Pellet 2, Figure V-43) with median particle size of $232 \mathrm{~nm}$ and to the last pellet (Pellet 3, Figure V-43), including the exosome fraction, with particle size of 143 $\mathrm{nm}$. Pellet 1 and 2 contained larger vesicles at a lower concentration, whereas pellet 3 contained higher concentrated, but smaller vesicles. 

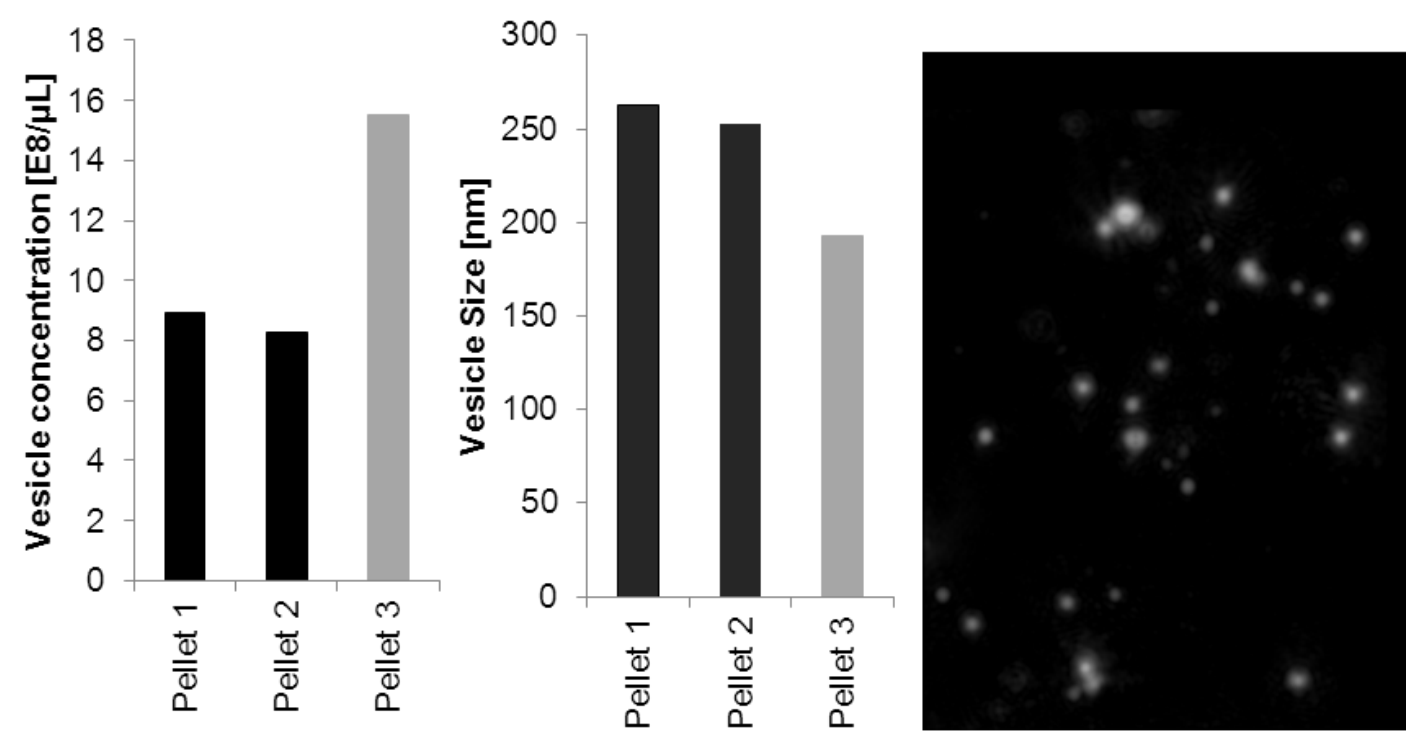

Medium and large vesicles in lower concentration

Small vesicles in high concentration

Figure V-43: Plasma and cell-derived vesicles from red blood cell units measured by NTA

After isolation of EVs and EXs from RBC units $(n=3)$, vesicle concentration at a final dilution of 1:500 (first diagram) and vesicle particle size (second diagram) were measured with NS500 (NanoSight, UK). Values are reported as mean. Black bars represent the medium (mean size of $228 \mathrm{~nm}$ ) and large vesicles (mean size of $232 \mathrm{~nm}$ ) at a lower concentration $\left(8.4-9.0 \times 10^{8} / \mu \mathrm{L}\right.$ ). The pellet 3 included smaller vesicles (size ranging from 65 to $192 \mathrm{~nm}$, mean size of $143 \mathrm{~nm}$ ) at a higher concentration of $16.0 \times 10^{8} / \mu \mathrm{L}$, shown as grey bars. Freeze image of a video representative for tracked vesicles (white dots) is shown on the right.

In this study, the vesicle composition of PCs and RBC units was investigated by sd-FCM, hsFCM and NTA. The results obtained from both flow cytometers, i.e. hs-FCM and sd-FCM, showed that PL-EVs constituted the main vesicle part of PCs, whereas RBC-EV concentration did not exceed the level of $2 \%$ during 5 days of the analysis. The L-EV amount measured in PCs turned out to be negligible.

Interestingly, with the help of flow cytometry, it was found that PL-EVs acted as a main vesicle part of RBC units. After preparation of RBC units, the vesicle quantification applied to vesicles from several pellets confirmed this thesis. Analysis by NTA allowed estimation of the size of prepared vesicles, but no immunolabeling was performed in order to determine the cell specificity. It remained unclear, whether PL-EVs were found in RBC units as a major subpopulation among all vesicles because of their resistant membranes, or due to the fact, that platelet vesiculation was more pronounced in comparison to vesiculation of RBCs during manufacturing of RBC units. 


\section{Discussion}

Red blood cell units, fresh frozen plasma and platelet concentrates are the most commonly administered blood concentrates. This study focused on platelet concentrates, obtained from apheresis donation, in order to improve their in vitro quality measurements.

Application of plateletpheresis concentrates (PCs) is an essential and life-saving treatment of patients undergoing a surgery, suffering from bleeding disorders or bone marrow failure syndromes. Platelets play a fundamental role in preventing vascular disintegration. In Germany, a consistent annual increment of PC preparation was investigated (263). More than 580,000 whole blood and apheresis platelet products were manufactured in the year 2012 (264). Compared with other blood concentrates, PCs have a relatively short half life and their durability was restricted to 5 days, including the day of apheresis. This fact results in the alterations of platelet function in vitro, namely, leads to the so-called platelet storage lesion (PSL) (17), which is characterized by reduced platelet recovery and survival in vivo (265). According to the best suitable description, PSL is a sum of all changes in platelet activity, its morphology, metabolic activity, signaling and functional features such as adhesion and coagulation in vitro. Weakened recovery and survival of platelets is frequently accompanied by bacterial contamination, which has an additional impact on the storage of platelets (266). Therefore, because of these obstacles, the production and consumption of PC products have to be balanced in order to guarantee a proper treatment of all patients.

In relation to the whole amount of manufactured PCs all over Germany, more than 490,000 concentrates were used in the treatment of thrombocytopenic recipients (264), therefrom 5588 at the University Hospital Regensburg. In order to prevent the application of low quality, nonsterile PCs or those of a non-fairly consistent dose, the preparation and storage of PCs underlay rigorous guidelines for approval and delivery according to the standard test and acceptance certificates in plateletpheresis manufacturing. Standard test are embedded in strict quality control (QC) programs with commonly accepted standards worldwide, allowing, however, minor deviations from manufacturer to manufacturer.

In order to improve the quality-defining analysis of $Q C$ in the view of current requirements referring to the influence of extracellular vesicles (EVs) on health and disease, this study implemented the investigation on platelet-derived extracellular vesicles (PL-EVs) from PCs as a quality control approach. Relevant fragments of results obtained in this study deal with emerging donor-related, preparation-related and storage-related differences within PL-EVs from PCs.

Vast knowledge on coagulatory and anticoagulatory functions of PL-EVs $(9,47,140)$ could be beneficial in evaluation of not only the physiological distribution of vesicles in blood circulation, but also of their distribution after application of PCs. However, until today, this aspect has attracted only a little attention $(5,15,228)$. It must be considered, that a certain amount of PL- 
EVs underlies changes within PSL and it is not obvious, whether and how the viability and activatability of platelets in stored PCs in vitro are affected by the vesiculation of platelets. It has also to be established how the coagulatory and anticoagulatory forces of PL-EVs in vivo after application of PCs are manifested. The assumed effects of PL-EVs on the changes in platelets during storage are transferable to other cell-derived vesicles within PCs (i.e. vesicles derived from red blood cells) and to other blood components and their storage lesions, for example red blood cell units. This aspect was considered as the last part of this study.

The most critical question in evaluating PL-EVs originating from PCs relates to the methodological issues dealing with detection of small particles by standard or new technologies. In order to facilitate the answer, the conventional flow cytometry (FCM) was validated as a gold standard. When detecting PL-EVs derived from fresh and altered PCs, the method, defined as a standard method (sd-FCM) by Navios ${ }^{\mathrm{TM}}$ (Beckman Coulter), proved to be reliable, stable and precise. In comparison to the scientifically present state of the art, the analysis of cell-derived EVs from several body fluids by sd-FCM has proved to be a qualified and satisfactory method (245). Further, sd-FCM analysis of PL-EVs originating from PCs was described as an assumably reliable method (15).

This knowledge provides the basis for consideration of the aspects of PL-EV analysis, the measurement of absolute vesicle concentration and the determination of vesicle size. It is beyond a doubt that vesicles can be determined in a reliable way, but it has not yet been clear if detected vesicles possess the expected size. The size of membrane-derived vesicles depends on the resolution of the flow cytometer and the calibration of the machine by beads with the physically distinct characteristics in scattering of light. The polystyrene beads used to calibrate sd-FCM by setting the vesicle gate, differ to membrane vesicles in a higher refractive index and, thereby, lower intensity of light is necessary to scatter light in the same way like cell-derived vesicles do.

It has been reported that due to the differences between refractive features of vesicles and beads, the captured cell-derived vesicles reveal a size which varies from this of polystyrene beads. In case, $0.5 \mu \mathrm{m}$ polystyrene beads are applied to the calibration of vesicle-gates, the cell-derived vesicle size corresponds to the size of $1.0 \mu \mathrm{m}(49)$.

The PL-EV analysis by sd-FCM with limited size detection and calibration with polystyrene beads, employed in this study, let assume that only PL-EVs and no larger platelets (PLTs), of approx. $1 \mu \mathrm{m}$ size were detected with the help of this method. Therefore, a new flow cytometer (hs-FCM, Apogee) of high resolution, that means of high sensitivity, was implemented in the PLEV analysis, that turned out to be able to detect smaller cell-derived vesicles of the equal size down to $400 \mathrm{~nm}$ (214). The assumption that the hs-FCM is able to recognize smaller vesicles was confirmed by the comparing results of the PL-EV-ratio measured by both systems. The ratio analyzed by the new system was 10-fold higher than the ratio of PL-EV to platelets 
measured by the standard FCM. It has to be kept in mind, that the high resolution for small particles does not comprise the capture of normal sized PLTs.

Furthermore, the additional technique, applied to determine the vesicle size with the use of the nanoparticle tracking analysis (NTA, NS-500, NanoSight) demonstrates that there is a surprisingly high amount of vesicles of decreasing size down to $50 \mathrm{~nm}$. These smaller vesicles also include lipid vesicles, protein aggregates and immune complexes. The recent knowledge and the results of the present study demonstrate that the size of PL-EVs is cell-specific measurable for a certainty down to $400 \mathrm{~nm}$ but not down to $50 \mathrm{~nm}$. More investigations with labeled PL-EVs, measurable by NTA, are crucial in order to satisfactorily solve the size issue. With regard to the detection of size by NTA, the validation of method results in obtaining high accuracy and recovery of both, monodispers and polydisperse polystyrene bead solutions. The results proving intra-assay precision in the determination of size (coefficient of variation [CV] of approx. $10.8 \%$ for PCs on day 7 ) and concentration (coefficients of variations up to $25.9 \%$ for PCs on day 7), were satisfactory.

Similarly, as in the case of the method which bases on the effects of physical characteristics of scattering light on flow cytometry, the NTA method uses scattered light for tracking illuminated vesicles and it calculates the size using the rapidity of movement due to the Brownian motion. Here, the scattered light of NTA rather limits the simultaneous analysis of high polydisperse body fluids or solutions of cell-derived vesicles. During the analysis, larger vesicles scatter more light than the smaller ones and smaller vesicles are underestimated by adapted instrument settings, i.e. low camera level. Conversely, large vesicle population is undetectable in high dilution and with the instrument settings for small vesicles. One of the inventors of the technique (Dr. B. Carr) pointed out, that particles of the same size, regardless of which material, i.e. gold or plastic spheres, exhibiting certain mathematical and physical properties, show the same Brownian motions under the same conditions (such as temperature or viscosity of suspension). Therefore, the question of measurement of cell-derived EVs may be answered using NTA for detection of accurate complementary size relating to the illuminated moving particles.

The question of EV-concentration could not be answered with the help of this method, because it was unclear, whether the camera was able to capture within one shot all vesicles in the continuum of size. The manufacturer's advice on the optimized determination of particle concentration in a solution (per $\mathrm{mL}$ ) was confirmed by results during validation of the method. In order to guarantee linearity in reproducibility of values, the recommended particle concentration ranging between $10^{8} / \mathrm{mL}$ and $10^{9} / \mathrm{mL}$ had to be achieved. As such, elution of larger particles must be mentioned again. However, during the first measurement, the particle concentration of a new sample is unknown. In body fluids and samples from PCs, the vesicle concentration varies individually and it depends on steps in sample preparation before analysis. Mostly, body fluid samples are highly polydisperse with vesicles in a size range of less or equal $50 \mathrm{~nm}$ up to $1000 \mathrm{~nm}$ and more. In order to capture all movements of the particles by NTA, a three stage 
setting should be used: at first, low dilution of the sample and lower incidence of light should be applied; secondly, medium dilution with medium incidence of light should be administered and finally, high dilution with a high incidence of light should be used to capture even the smallest particles, which scatter less light due to their size. This measurement record proved to be the best one employed in order to acquire a high reproducibility of data, however it also turned out to be time-consuming.

In the first tests with immunolabeled PL-EVs using NTA only for a short time, i.e. for less than 2 seconds, PL-EVs were visualized in the fluorescent mode by NTA. Detected plasma vesicles from PCs are likely to contain PL-EVs, but the green laser bleached the conventional fluorescent monoclonal antibodies, i.e. CD61-PC7. This effect occurs due to the light exposure which stimulates the molecules into fluorescing, but otherwise diminishes them. Although, it has not been mentioned in this study, the labeling of vesicles with sypro-red dye which stains proteins and glycoproteins as well, was stable for minutes and showed a huge amount of intact cell-derived vesicles. It is important that more investigations employing photobleaching-stable and cell-specific fluorochromes and the use of a syringe pump for very long captures should be carried out in order to analyze the absolute PL-EV concentration by NTA. Without a plateletspecific labeling and without additional preparation steps, as density gradient centrifugation of prepared exosomes, determination of vesicle size and vesicle concentration by NTA is not completed. Although, the proof of linearity of plasma vesicle concentration from PCs by NTA was demonstrated, the fact concerning the lack of specificity of PL-EVs provokes more investigations and prompts questions of the discrepancy between PL-EV concentration measured by hs-FCM (Apogee) and plasma vesicle concentration from PCs analyzed by NTA.

Regardless of the fact, whether blood samples or PC samples were analyzed, the concentration of PL-EVs was controversially documented in literature (see Table VI-1). Differences were reported not only among several flow cytometers, but also between flow cytometry as a method and other detection techniques, i.e. atomic force microscopy (AFM) and electron microscopy (EM), to name a few. In this study, the standard method of PL-EV-analysis was a semiquantitative method with an indication of a PL-EV ratio according to the platelet events. As depicted in Table VI-1, the PL-EV ratio amounting to approximately six percent on day 1 was similar to the PL-EV ratio reported in literature. The concentration of CD61-positive PL-EVs measured by semiquantitative method was not comparable with the absolute concentration of CD61-positive PL-EVs measured with hs-FCM. Nevertheless, the reported PL-EV concentration in PCs also varied even if measured with the same hs-FCM method (256) and there is no common pre-analytical standard applicable to the analysis of PC samples. A standard protocol of EV analysis in samples stemming from several body fluids has been recently presented (267). This protocol was revised in the current study and was successfully employed in a running metacentric study conducted in four transfusion centers for QC of PCs. 
Table VI-1: Comparison of PL-EV concentration from PCs on day 0 or day 1 reported in recent literature

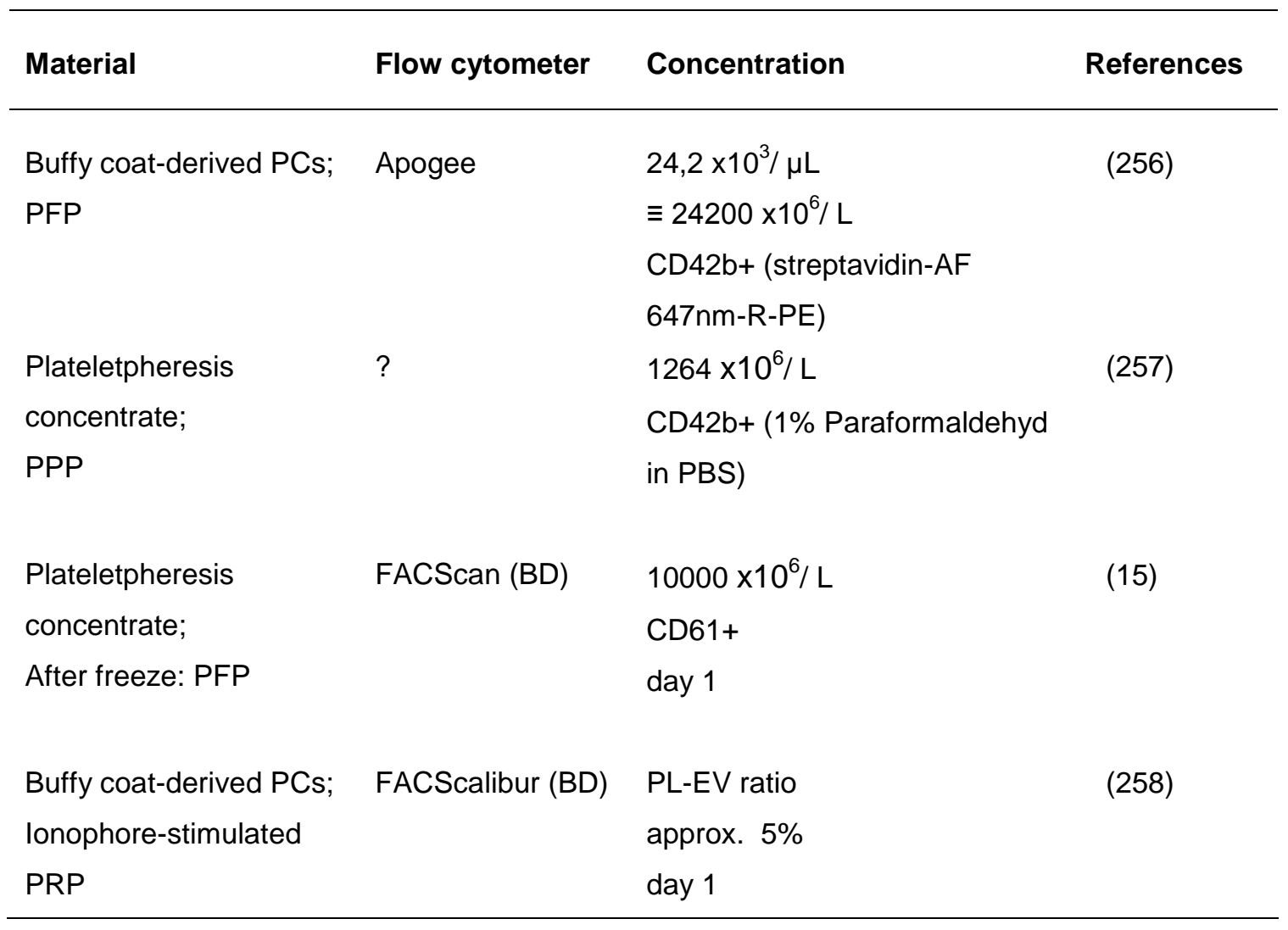

The semiquantitative method could be a useful parameter of the comparison of the results of a metacentric study because the performance with sd-FCM may allow the most centers to participate in this investigation. Further, the PL-EV ratio may be acceptable as a quality control parameter because the platelet events determined by sd-FCM correlate to the platelet count measured by an automated blood cell counter (Sysmex); no significant changes in the count during storage during 5 days were observed. Therefore, the change of the PL-EV-ratio during storage, despite of the stable PLT count, may be the earliest and quickest test for a standardized detection of vesiculation in a QC program. In fresh, non-irradiated PCs, the PL-EV averaged 6.1 percent in contrast to the mean value of 7.1 percent for PL-EVs in PCs after expiry. In irradiated PCs, the values averaged 6.8 percent on day 0 and 11.8 percent on day 5 (that means day 6 after preparation), confirming the QC test. It is not clear, whether the higher vesiculation rate during storage affects the viability of PLTs after application of PCs in vivo, but coherence between PL-EVs and the activatability of PLTs is very probable. The effects of storage on the externalization of $p$-selectin (CD62P) at the surface of PLTs are described in detail later. 
Further, investigations of sd-FCM with count beads are recommended in order to compare the results obtained with quantitative methods of PL-EV analysis. As a means to confirm linearity, small-count beads, exhibiting the most similar characteristics to membrane vesicles, i.e. silica beads, could be applied instead of physiological, cell-derived reference control beads for spiking samples. With the purpose of improving the method, it has to be mentioned, that the chosen monoclonal antibody CD61 binds in a reduced form to HPA-1b (human platelet antigen-1b), which is one of the expressed forms of the two P1A alleles of the glycoprotein (GP) Ilb/llla gene (268). This results in stronger mean fluorescence intensity (MFI) of the P1A1/P1A1 variation in platelets and less MFI values of the P1A1/P1A2 polymorphism. The CD61-positive events of vesicles and platelets analyzed by this method originate from the same donor and, therefore, it is assumable that PLTs and PL-EVs show the equal CD61 expression at their membrane surface, without interference with the PL-EV-ratio.

PLTs are known to convey cytoplasmic mRNA as a molecular tool for protein biosynthesis and they are able to synthesize GPIlla during 7-day storage (269). In case, PL-EVs containing mRNA are not able to synthesize proteins in amount equivalent to the amount of proteins synthesized by platelets, the volume of PLTs for semiquantitative CD61 positive PL-EV-ratio analysis will be overestimated, in contrast to the volume of PL-EVs from stored PCs. Moreover, integrin signaling, which influences protein biosynthesis during storage of PCs, indicates a link to the pathways of PSL (269). During the storage of platelets, alterations in talin, tubulin and thrombospondin were found $(30,270)$. In respect to the microparticle formation, generally and specifically for PL-EVs, talin, as degraded cytoskeleton molecule, beside myosin, and Rasrelated protein 1 (Rap1) effector RIAM (Rap1-GTP-interacting adapter molecule), plays an important role in an agonist stimulation in integrin allbß3 binding and activation (271). Additionally, Rap1 activation was diminished in an inhibitor-induced study targeting PI3-kinase, accompanied by reduction of integrin allbß3 activation and release of $\alpha$-granules $(22,272)$. Therefore, it is assumed that the agonist stimulation of platelets, leading to integrin activation and Rap1-induced formation of an integrin activation complex, is involved in vesicle development in apoptosis or alteration during storage.

As far as an optimized QC analysis is concerned, the correlating proteomic data, i.e. data on increase of cytoskeleton element talin, vinculin and moesin, as well as on signal transduction proteins 14-3-3, RhoGDI (Rho GDP-dissociation inhibitor) and Rap1, were suggested as a profile to predict PC quality (273). In this research, the decrease of these proteins translated to a poor platelet recovery and survival. In addition to protein profiling analysis and a research aiming to avoid expensive und extravagant radiolabeled survival-tests in vivo, the PL-EV analysis and, furthermore, proteomic data on PL-EV study should be integrated in a forwardlooking QC process.

Surface antigens on PL-EVs such as CD41, CD42b, CD63, annexin V, phosphatidylserine (PS) and cleaved filamentin A $(9,256)$ were also detected during PL-EV analysis, however, the 
antigens could not be detected with the standard method applied to this study. First measurements permit to remark, that 50-60 percentage of all PL-EVs are annexin V-positive. Further investigations to enclose the potentially changing immunophenotype of PL-EVs by a day-by-day analysis for QC tests are necessary.

Inconsistent results gathered from different NTA and FCM methods, carried out either with fresh PCs or with irradiated PCs after expiry, were also expected. Interestingly, Canto ${ }^{\mathrm{TM}}$ II, a standard FCM method similar to Navios ${ }^{\mathrm{TM}}$, was able to recognize silica beads according to the forward scatter (FSC) size determination down to $240 \mathrm{~nm}$. As a result of the comparison of the three FCMs (Navios ${ }^{\mathrm{TM}}{ }^{\text {, Canto }}{ }^{\mathrm{TM}}$ II and Apogee) and samples from PCs on day 4, similar PL-EV-ratios were obtained in an intra-assay test with CV about $6.2 \%$. The results of the analysis of the samples from the seventh day exceed the acceptance criteria (set to $\leq 15 \%$ and they amounted to $\mathrm{CV}=16.2 \%$. On day 7, Apogee (hs-FCM) detected more vesicles than the other both flow cytometers. This can be explained by the fact that the amount of smaller vesicles grows after expiry. Moreover, the vesicles are platelet-specific and detectable only by hs-FCM with a resolution for PL-EV smaller than $240 \mathrm{~nm}$. Results of NTA analysis confirmed the thesis; the mean size distribution does not reflect the increased amount of smaller vesicles, though. The changes of PL-EVs during the relevant time of applicability (day 1 to day 4 ) have to be analyzed separately based on the validated protocol for PL-EV-measurement.

Before PL-EV analysis was performed with the use of fresh samples from PCs and samples after expiry, the thesis on platelet activation in relationship to vesiculation had been confirmed. In response to the activation by thrombin-receptor-activating-peptide 6 (TRAP-6) or by application of shear stress as a second mechanical activator (induced by a strong sampling), of a separated group of PC samples, an increased shedding of PL-EVs from platelets was induced. Activation by TRAP- 6 generated an average increase of $1 / 4$ more PL-EVs in fresh PCs than in the same PC samples without mechanical activation. Beside the strong agonist, TRAP6 , shear stress is also known as a platelet activator that induces vesiculation (230). The data on PL-EVs in this study showed no statistically significant differences between the activated and non-activated platelets of different sampling types, but the results demonstrated clear differences in the correlation between PL-EVs and the CD62P expression after activation with TRAP-6. CD62P was used as a gold standard to show the activatability of platelets. On the one hand, PL-EVs positively affected the activatability of unstressed platelets and, on the other hand, there was a negative effect influenced by the rigorous type of a sampling. The results, collected within 144 hours after sampling, were completely opposing between activated and non-activated platelets and it is likely that activated platelets underwent shape change accompanied by an increased vesiculation that negatively influences the activatability. Activated platelets were not able to reach the resting state as in case of the platelets in unstimulated samples from the same PCs. Results from the rebound phenomenon test let suppose that, despite shear stress during production, the functionality of platelets recurred. Further, the more 
PL-EVs are shedded after 24 hours, the more positive relationship with the platelet function is assumable in samples not subjected to shear stress. By contrast, more PL-EVs and platelet function is assumably negative related in samples with stressed platelets. Inadequate activation of platelets during manufacturing process leads to reduction of their functionality accompanied by a higher PL-EV amount, especially four hours after sampling.

According to the QC standard, samples with abnormally high CD62P values and a higher amount of PL-EVs on the day of apheresis imply lower quality of platelets in PCs. PL-EVs are useful as plausibility check in QC. This confirms the thesis, that there are different ways of platelet vesiculation within different types of PL-EVs and resting platelets exhibit less vesiculation (15). It remains obscure, whether PLTs originating from different manufacturing technologies vary in their in vitro functional activities (274). Additionally, it would be of great interest to investigate, whether PL-EV functional activity might be independent on their concentration. More researches applying other agonists are necessary to be conducted with a purpose of detection other ways of vesicle formation from platelets, indicative of different functionality of PL-EVs.

With the help of impedance aggregometry used in this study, it was possible to confirm the results reported in 1967 by Wolf (8). The PL-EVs were originally called "platelet dust" and it was possible to prove their coagulant properties with the help of the clotting time measurement. Recently, it has been also possible to demonstrate by means of Impedance Aggregometry that PL-EVs cause aggregation in platelet-free plasma (PFP). Even platelet-derived exosomes (PLEXs) exhibited this phenomenon. However, the control samples, EV-free plasma alone or PBS manifested no coagulant properties and did not provoke any measurable aggregation. Results showed significantly increased aggregation in PL-EV-rich samples in comparison to the control. Therefore, the method of aggregometry has been rejected as a method of improved PC quality control. Different components of PCs, platelets, plasma and PL-EVs are not distinguishable in relation to function by this method and the results of aggregometry do not imply the activatability of platelets. Aggregometry does not reflect specific platelet activation because of the artificial procoagulant activity of PL-EVs.

The donor-specific and preparation-specific values include the most relevant results that showed coherence to the PL-EV amount from PCs. No gender-, or age-related differences of PL-EVs were recognized, neither on day 0 nor day 5, confirmed by the recent results reported in literature (15). Moreover, the age of donors might positively affect the CD62P expression on platelets on day 0 . Subsequent positive coherence was demonstrated between the CD62P expression and BMI (body mass index) for both, CD62P values at the time after apheresis and at the time after expiry. Interestingly, this phenomenon was accompanied by a negative correlation to PL-EVs after expiry. The high BMI, in general, is parallel to elevated cholesterol levels and elevated LDL-levels. However, as it negatively correlates to the amount of PL-EVs, the relevant coherence between the BMI and the PL-EVs is questionable. Nevertheless, 
elevated cholesterol and LDL levels positively correlated to the CD62P expression. These results disprove the significantly higher BMI values found in a low-responder group for PLT responsiveness of activation in contrast to midrange or high-responder groups reported by Garner et al. (275). Mean BMI of the low-responder group accounted for 30.1 in contrast to the mean BMI of 24.5 found in the course of this study. Within the cohort of healthy donors, the lowresponder group was characterized by a minimum of platelet response after stimulation with adenosine diphosphate (ADP) and cross-linked collagen-related peptide. Fibrinogen binding and CD62P expression were determined as activation markers. It is likely that there is no coherence between BMI values and platelet activation due to the fact that the cohorts differ too much in their BMI values. However, in fact, BMI as a standard value of case history and clinical examination before apheresis could be useful for estimation of activatability of platelets from PCs.

Another parameter, also called the immature platelet factor (IPF) of donors' pre-donation samples, was discussed. Immature, young or so-called reticulated PLTs containing residual RNA are present in circulation after release from megakaryocytes in bone marrow. This parameter reflects the stage of megakaryocyte proliferation. A significant negative relationship between IPF and PL-EVs is assumable on day 0 , whereas positive relationship among IPF, PLEV and platelet count was observed on day 5 . The negative relationship between IPF and PLEVs within fresh PCs may demonstrate a high turnover and younger PLTs with either less vesiculation of PLTs in the peripheral blood or more viable, activatable PLTs pooled in the spleen (275). The coherence in altered PCs may indicate that some donors have particularly susceptible PLTs that are more viable during storage and are more capable of vesiculation in contrast to the apparent genetic determinants influencing modification of PLT function.

It is also worth discussing whether all PC-derived CD61-positive vesicles originate from platelets or from megakaryocytes of plasma of healthy volunteers (276). This would result in coherence between IPF and PL-EV amount in the donor's plasma, but it still cannot explain the negative relationship between IPF and PL-EVs from PCs. More investigations are necessary in order to characterize the phenotype of PL-EVs.

As far as the preparation-related differences within PL-EVs are concerned, very interesting results were obtained for single-needle and double-needle systems. As a result of the application of single-needle-system during apheresis process, a lower activatability of platelets and decreased CD62P expression on day 0 and on day 5 were noticed. Further, an increased citrate concentration and, consequently, a decreased $\mathrm{pH}$ within PCs were evaluated because of the higher demand for anticoagulation when apheresis time was shorter, when single-needle system was used. Neither single-needle nor double-needle systems affected PL-EVs statistically significant; however, a typical imbalance in PLT count occurred. Application of double-needle system of lower flow rates requires longer time of apheresis; yet, the resulting collection of platelets above the buffy coat is likely to be more effective and leads to higher PLT 
count. Aggregation of PL-EVs and forming larger particles, and detection of false positives within platelet populations may explain why both apheresis systems generate similar amount of PL-EVs in PCs. However, higher citrate consumption in concentrates obtained in the course of single-needle procedure speaks against aggregation of PL-EVs.

The rebound phenomenon tests did not confirm any discrepancies within PC-derived PL-EVs, which were subjected to shear stress of varied intensity and manufactured by single-needle or double-needle systems (5.1.5). Additionally, PL-EV populations did not significantly differ from one another, however, the PL-EV concentration negatively correlated to CD62P expression in samples drawn under greater shear stress. Therefore, PL-EVs are not applicable as a single parameter of QC; rather they may serve as a marker of the plausibility check of platelet activatability.

The variation within PL-EVs could be evaluated solely with the help of instruments employing double-needle system. With regard to different cell separators, it was previously shown that the instruments variably influence induction of PLT activation (277) and longer processing times are associated with increased activation. Among all instruments applied, there was only one separator whose measurements indicated considerably higher levels of PL-EV ratio on day 0. The amount of processed PCs was equal. Provided, that PL-EVs originate from the platelets enclosed within PCs, only, and not from the donor plasma obtained during apheresis (15), specific settings of apheresis apparatus affect the activation of platelets through shear stress or another non-physiological, mechanical stress. In the case of this particular instrument, the CD62P expression was decreased after TRAP-6 activation, accompanied by elevated levels of PL-EVs. Therefore, with regard to the quality control (QC) management, PL-EVs are applicable as an indicator of unfavorable apheresis settings and, consequently, lower activatability of platelets, originating from PCs. Therefore, prior to the launch of the method applying PCs, further research on the influence of the aforementioned instrument on other QC parameters is essential.

Commonly accepted quality defining criteria for PCs, i.e. platelet count, sterility on day 5 or $\mathrm{pH}$, do not exceed the reference levels for the approval of concentrates used in this study. Only one PC exceeded platelet count defined by the reference levels and, thus, it did not meet the requirements. Therefore, the quality of PCs used in this study is acceptable. The expression of CD62P on day 0 averaged 1094.7 percent after activation with TRAP- 6 and it dropped to 547.3 percent on day 5 after activation with TRAP-6, as compared with the control values. CD62P expression at the platelet surface is one of the most common markers of platelet activation in PCs and there were standardized protocols established to compare the activation of platelets during storage with increased CD62P level over time (37, 278-280). Reported in literature, data on increased CD62P level, not influenced by an additional activating stimulus, were obtained from partly fixed platelets, not stimulated by TRAP-6. Our data confirmed these results of increased basal CD62P values during the storage. Until today, no in vitro test has received 
exclusive approval as a standard test. In general, it is accepted that agonists induce alterations in platelet shape and surface antigen expression. They are useful for protocols on platelet function tests in QC of PCs $(5,281)$.

In the present study, this criterion was fixed as a gold standard for the evaluation of PL-EV levels as a quality control approach.

Unlike the level of PC-derived plasma vesicles measured by NTA, the concentration of PL-EVs evaluated by flow cytometry has been revealed in the recent literature (15). The concentration was computed with the help of a formula by Berckman (86) employing PL-EV concentration of approx. $10000 \times 10^{6} / \mathrm{L}$ on day 1 and approx. $7500 \times 10^{6} / \mathrm{L}$ on day 5 (data of CD61 positive MP events obtained on the base of published figures, not written-out). Another study, reported in literature, demonstrated values of PC-derived PL-EVs labeled with CD42b moAbs. The concentration of PL-EVs in PCs reached the level of approx. $1264 \times 10^{6} / \mathrm{L}$ and the level of approx. $1702 \times 10^{6} / \mathrm{L}$ on day 0 and day 5 , respectively. In order to compare the data aforementioned and data from the current study, numerous aspects have to be discussed.

At first, the formula by Berckman is not applicable to the semiquantitative PL-EV analysis by Navios $^{\mathrm{TM}}$. Secondly, the PL-EV concentration analyzed by hs-FCM (Apogee) corresponds to the presence of small, medium and large-sized vesicles ranging from $180 \mathrm{~nm}$ to $500 \mathrm{~nm}$. PL-EV concentration measured by hs-FCM in this study was 450 fold higher $\left[4509150 \times 10^{6} / \mathrm{L}\right.$; $10000 \times 10^{6} / \mathrm{L}$ ] than the concentration obtained by Rank et al., and more than 3500 fold higher than the PL-EV level reported by Nollet et.al. (257). This indicates that there are differences in statements of PL-EV concentration in PCs varying from approx. 1000 to millions of PL-EVs per liter. Of course, different instruments were used in analysis by FCM, but until today, it has conveyed an impression of measuring ballpark figures. The values of PL-EV concentration of the largest subpopulation ( $300 \mathrm{~nm}$ to $500 \mathrm{~nm}$ ), as determined by hs-FCM in this study, are not comparable to the results obtained by the aforementioned research groups, either. Further investigations are necessary to standardize techniques and methods of analysis of PL-EVs from PCs. However, the high resolution flow cytometer is likely to separate much more PL-EV vesicles than previously predicted. Even with the help of the analysis with optical single particle tracking, it is allowed obtaining higher concentrations of vesicles within PCs.

Concluding, the PL-EV concentration, exemplarily on day 0, measured by hs-FCM [approx. $4500 \times 10^{6} / \mathrm{mL}$ ] constitutes 1.5 percent of the total amount of plasma vesicles determined by NTA [approx. $300000 \times 10^{6} / \mathrm{mL}$ ]. This result is comparable with the results acquired from PFP, labeled with cell-tracker dye and two decimal power lower cell-derived vesicles, which are in fluorescent modus versus all measured plasma vesicles (248).

The size of cell-derived vesicles from PFP ranged from $50 \mathrm{~nm}$ to $300 \mathrm{~nm}$ with a peak size around $80 \mathrm{~nm}$. According to a general expectation that the majority of vesicles in human plasma derive from platelets $(15,142)$, these results conform to the size of PL-EVs. Another group reported that PL-EVs are expected to be of a size of approx. $125 \mathrm{~nm}$ in diameter as measured 
by atomic force microscopy (282). Although, the average size of the plasma vesicles amounts to approx. $150 \mathrm{~nm}$, it does not refer to the size of PL-EVs. Additionally, Dragovic et al., assumed, that the majority of plasma vesicles are lipid vesicles (248), which may influence the dimensions of the structures constituting PCs. Even some additional EV particles, i.e. high-density lipoproteins (HDL), cannot be isolated from EVs by means of sucrose gradient ultracentrifugation, due to the fact that their densities overlap (283). Large protein aggregates, which constitute other contaminating structures in plasma-containing samples, are not distinguished from PL-EVs by NTA based on the phenomenon of Brownian motion (267). In fact, the current literature does not provide enough information and no consensus on the exact size and concentration of PL-EVs from PCs determined by hs-FCM and NTA. This study implies existence of platelet-derived vesicles, stained with CD61 moAbs, of a size between $180 \mathrm{~nm}$ and $1 \mu \mathrm{m}$.

Further, two groups of PC samples were compared for QC testing. Samples from tubes from the apheresis container, filled with PC content on the day of preparation, and samples from the whole PCs constituted the investigated groups. The results of the comparison demonstrate that the material from the tubes is not useful for $Q C$ analysis. When comparing the data on platelet count, CD62P expression on platelets and on the amount of PL-EVs, it was noticed that the samples originating from the same platelet concentrate differ from one another what is likely to be caused by the presence of inadequate mixture of the tubing system. As a result, the equivalence was rejected. However, standardized stripping prior to sampling process may prevent the rejection. Extreme discrepancies in results of the material originating from the tubes, obtained for the latter specified parameters, reflect this assumption. Flexibility in standardized sampling of QC analysis would prevent the destruction of worthwhile pharmaceuticals. The process needs further validation and approval by regulatory authorities.

In order to fulfill requirements for the evaluation of qualified PC samples corresponding to the real content of PC, the results of PL-EV analysis were interpreted only on the base of samples of the whole PC.

The present study investigated a potential effect of gamma irradiation on platelets originating from fresh PCs and on vesiculation and PLT activation. This issue appeared to be more interesting than expected at the beginning of this research, due to the fact that, more than 50 percent of the administered PCs at the University Hospital Regensburg underwent irradiation in the past year. Gamma irradiation of 25 Gy exposed to PCs prevents the proliferation of leukocytes before transfusion $(284,285)$. This process reduces the potential life-threatening transfusion associated graft-versus-host disease (TA-GvHD) in immunocompromised patients. Provided that RNA-containing platelets and even PL-EVs translate functional proteins during alteration, UVA gamma irradiation may inhibit PSL-linked protein production. With the help of PL-EV proteomic analysis by LC-MS/MS it has been discovered that five percent of proteins involved in molecular function belong to transcription and translation processes (286). Taking 
into consideration that the aim of this study is to obtain long-lasting products, the methods of protein and PL-EV analysis are certainly subject for improvement. Results presented in the current literature do not confirm any obvious changes in platelet count observed between irradiated (with 20 to $25 \mathrm{~Gy}$ ) non-irradiated platelets $(29,280,287)$, but results obtained in the course of this study show significant differences in the platelet count between the two groups on day 0 . The dosed gamma irradiation used in this research was constant and not subject to change. The fact, that diverse blood cell counters (i.e. ModelZM, Coulter; Cell-Dyn 4000, Abbott; analyzer from Rock et.al. not known) were applied to three mentioned studies, is likely to be a reason of discrepancies between the results obtained in the aforementioned studies and in the analysis by Sysmex XE-5000. Generally, an analysis of platelet count performed with automated hematology analyzer is validated with the help of blood samples but rarely validated with the help of samples from PCs. PLT count analysis of PCs with Sysmex XE-5000 was based on the earlier validation performed with samples originating from PCs. The evaluation of platelet count of irradiated PCs versus non-irradiated PCs during monthly QC analysis has to be continued. However, platelet count performed by hs-FCM approved the results of elevated platelet count after irradiation on day 0 .

Beside the change of the CD62P expression, no differences were found between irradiated or non-irradiated PCs, confirming the common assumption reported in the recent literature (29, 280). The results of platelet vesiculation in PCs after $y$-radiation have not been presented in the literature. Nevertheless, it was reported that human and mouse lung cancer cell lines subjected to irradiation with 10 Gy secreted up to four times more microvesicles (MVs) after 24 to 48 hours without affecting the viability of cells (288). These cells are nucleated in contrast to platelets, which remain unaffected by irradiation. However, the data suggest an influence of irradiation on the amount of PL-EVs. The sd-FCM and the hs-FCM showed significantly altered PL-EV ratio after irradiation of PCs.

In aspect of vesiculation depending on storage effects to platelets, with the help of hs-FCM it was possible to determine the differences of PL-EV amount accompanied by lowered PL-EV ratio on day 0 , whereas the results of sd-FCM showed significant changes after expiry of the PCs only, in form of elevated PL-EV ratio. These results, however, are not quite contradicting one another. The grow of platelet amount should lead to lower PL-EV/ PLT-ratio as measured by hs-FCM on day 0 in contrast to not significantly elevated $(n=15)$ or stable $(n=30)$ PL-EV/ PLT-ratio as determined by sd-FCM. This discrepancy on day 0 is based on the overestimated or underestimated platelet count by different FCM instruments, bearing in mind that the sd-FCM is a semiquantitative method. Platelet events detected by sd-FCM, are 10-fold more frequent than PL-EV events, whereas the platelet events detected by hs-FCM are only twice that frequent than PL-EVs. Changes in platelet events, measured by hs-FCM, lead to the fact that the PL-EV ratio is more susceptible to alteration. Latest results do not include the comparison of PL-EV ratio (estimated by hs-FCM) between non-irradiated and irradiated PCs on day 5. 
However, it is supposed that the absent data would confirm the elevated PL-EV ratio measured by sd-FCM during storage. Anyway, the concentration of the main vesicle fraction, the lipid vesicles, does not change after irradiation of fresh PCs. In conclusion, it has been noticed that the platelets vesiculation is the sole phenomenon, which could explain adverse effects after transfusion of irradiated PCs. PL-EV ratio is the only indicator which changed after irradiation and more investigations of this topic are necessary to estimate modifications after irradiation of PCs with the hs-FCM and labeled PL-EVs by NTA.

In order to prove the quality of the storage conditions of PCs, e.g. the storage temperature, agitation, storage containers, solution and anticoagulants, the measured stable platelet count was used as a positive, predictive indicator of proper conditions The platelet count is one of the variables of approval and delivery according to the standard test and acceptance certificates in plateletpheresis manufacturing. These results were reported earlier and stay consistent with our data $(25,280)$. The $\mathrm{pH}$ values, which correspond to the metabolic processes under nonphysiological condition during storage after removing PLTs from the circulation, also match the reported data (279) (5). In this study, $\mathrm{pH}$ does not exceed the reference level at any time of storage. Our data on expression of CD62P on platelets during storage are also conformed to general findings. Disclosed data mainly demonstrated increased level of basal CD62P surface expression (37, 278-280), observed in platelets which were not stimulated and their activation is solely a result of the non-physiological conditions in storage container. The initial increase of CD62P was confirmed by our data; nevertheless, it was of interest to search whether the activatability of platelets at the beginning and the end of storage may serve as an applicable indicator of in vivo viability. In another study, similar changes of CD62P expression after TRAP6 activation during storage of PCs over 12 days were recognized, even accompanied by a complete loss of responsiveness after that time (258). The loss of platelet activatability during five days of storage time obviously appeared and did not depend on irradiation of PCs on day 0 . This loss of activatability and an increased basal CD62P exposure indicate one of the effects called platelet storage lesion and this effect served as a gold standard of functionality. Therefore, it is plausible to employ the standard as a basis for evaluation of PL-EVs as a QC approach.

PL-EV increased also during storage, independently of the irradiation. In literature, there are discrepant results. Decreased level of PL-EVs labeled with CD61 moAbs (15) was reported as well as increased concentration of PL-EV labeled with CD42b moAbs (257) and increased level of PL-EV labeled with CD61 moAbs (258) over time. In our study, the analysis by hs-FCM confirms the results by sd-FCM at least, as far as elevated PL-EV ratio during storage in irradiated PCs is concerned. Further, the PL-EV ratio on day 0 , as determined by sd-FCM, correlates to the PL-EV ratio from the fifth day. This may indicate that the PC-derived platelets undergo vesiculation already at the beginning of storage and this notion could facilitate a decision whether specific PCs should be applied in a therapy prior to other PCs. 
Plasma vesicle concentration determined by NTA does not change during storage, as expected, due to the fact that PL-EVs translate to 1.5 percent of all plasma vesicles measured in PCs.

The increase of PC-derived IPF is another noticeable change during storage. This phenomenon stays consistent with previous data and was explained by mainly unspecific binding of nucleic acid dyes to the membrane surface of platelets or residuals of mRNA (25). In addition, the fact that similar results of IPF increase and stable platelet count over time were acquired, the interpretation of the data of this study allows suggesting coherence between elevated IPF values and increased PL-EVs in PCs. This coherence could be explained based on the supposition that PL-EVs contain nucleic acid, i.e. RNA that is labeled similarly to IPF and, thus, provides false results. Furthermore, it was demonstrated that there is a relation among IPF, PLEV and CD62P on the fifth day of storage, only; further analysis is required, though.

The correlation between the expressions of CD62P, employed as a QC gold standard of platelet activatability during storage and PL-EVs from the PCs was in focus. The question was whether PL-EVs might be useful as an indicator of the platelet activatability.

According to the results obtained, PL-EVs were confirmed as a marker of fresh, non-irradiated PCs and irradiated PCs after expiry on the fifth day as determined by sd-FCM. With knowledge of several experiments described in literature in last years, it is assumable that sd-FCM used in this study distinguishes the size of PL-EVs of approx. 0.5 to $1 \mu \mathrm{m}$ (214).

First, the sd-FCM without high resolution is able to determine particles of approx. $300 \mathrm{~nm}(246$, 247 ) and secondly, the vesicle gate for the tests was adjusted to smaller or equal size of $0.5 \mu \mathrm{m}$ calibration beads consisting of polystyrene. It is conceivable that the PL-EV analysis by sdFCM, performed in this study, covered more microvesicles than exosomes derived from platelets. Otherwise, one cannot rule out the possibility that vesicles of a size of exosomes are detected as 'swarm within the iceberg' $(49,255)$.

The results of correlation and linear regression are significant for the coherence between PLEVs and CD62P in fresh, non-irradiated PCs showing a considerably lower expression of CD62P, after activation with TRAP-6, reaching approx. 51.5 percent due to the 1 percent increase in PL-EV ratio. The significantly lower activatability of platelets from irradiated PCs after expiry reached approx. 16.9 percent in parallel to 1 percent increase of PL-EV ratio. The same negative correlation between TRAP-6 stimulated CD62P and PL-EV amount was previously evaluated, but it was of no significance (258).

What are the reasons for this coherence? We know that the platelet count was stable during five days of storage and the count itself is not responsible for changes in PL-EV ratio. Further, we know that PL-EVs are likely to originate from platelets, accumulated in the storage container, but not from the donor plasma gathered during preparation (15). Thirdly, platelets of PC origin change their shape upon activation, produce more vesicles through blebbing and form less vesicles under the condition when platelet activation inhibitors are added to the storage 
containers (14). Fourthly, during storage, the PC-derived PL-EVs are less involved in apoptosis than in activation (258). Finally, the loss of responsiveness to TRAP-6 and reduced CD62P expression is a sign of exhaustion, affected by secretion of granules after activation and senescence or early apoptosis. This exhaustion was demonstrated in fresh activated platelets which were present under non-physiological and extracorporeal conditions. This may lead to a conclusion that PL-EVs in PCs provide a helpful predictor of platelet responsiveness. To summarize, one reason for the negative PL-EV/ CD62P coherence is the degranulation of activated platelets. Activation starts with response of platelet receptors to agonists, with crosslinking of receptors or changes of membrane integrity. Activation signaling occurs as 'outside-in' and 'inside-out' signaling (289) and is associated, among others, with secretion of granules and membrane-derived vesicles. Former activated platelets form vesicles and are less responsive to reactivation.

Moreover, nowadays, the interest in identification of signs of PSL process during storage in PCs is not limited to the amount of derived vesicles only. Proteomics of PLTs has gained importance as a tool to address pathways involved in the loss of morphologic, biochemical and functional changes during storage of blood products (269). Recently it has been found that approximately three percent of the cytosolic PLT proteome underwent changes within nine days of storage (20). An increase in the concentrations of septin 2, B-actin and gelsolin was observed, however the proteins were not among those 587 proteins which were identified in PL-EVs (290), research on http://evpedia.info/). Nevertheless, it may happen that the changes in proteomics, found in platelets during storage, might be also identified in stored PL-EVs.

One of the aims of the present study was to prove with the help of the obtained results the speculated relationship between the platelet activatability and the vesicle production. Additionally, new systems of PL-EV analysis with high-resolution FCM (Apogee) and the optical single particle tracking analysis (NTA) were favored as recent supporting techniques in the QC program of PCs. The results of PL-EV analysis by sd-FCM were compared to the results of both new systems. As far as both cytometric methods (the semi-quantitative (sd-FCM) and quantitative analysis (hs-FCM) are concerned, the ratio of PL-EVs to platelets was calculated based on the positive labeled CD61 events. The FCM methods delivered outstanding results comparing one another, which demonstrated a positive correlation of PL-EV ratios to CD62 expression in all analyzed samples from fresh PCs. There was a significant coherence between the non-irradiated $\left(\mathrm{cc}=0.72^{\star *}\right)$ and the irradiated PCs $\left(\mathrm{cc}=0.80^{* *}\right)$. In contrast, no significant coherence was detected between both systems determining the amount of PL-EVs after a storage period of five days under blood banking conditions. The fact that a considerable coherence has not been observed might be controversial because other, additionally acquired by a new system, parameters correlated very well. These parameters refer to the concentration of PL-EVs as CD61-positive events per $\mu \mathrm{L}$. 
The hs-FCM can distinguish among several subpopulations among PL-EVs from PCs. No attention was paid to the fraction of small PL-EVs with diameter lower than $180 \mathrm{~nm}$. It is uneasy to separate the events from the background noise and this may be one of the subsequent reasons for a low concentration of small PL-EVs and for an attained detection limit for human membrane vesicles with a refractive index of 1.39 in the size of $180 \mathrm{~nm}$ (214) personal communication with $\mathrm{O}$. Kenyon). Despite the fact that the PL-EV ratio failed to reach a significant correlation between the standard and new systems for expired PCs, the concentration of all PL-EVs from PCs and large PL-EVs (300 to $500 \mathrm{~nm}$ ) showed significant coherence to the PL-EV ratio as assessed by standard system for PCs applied regardless of the maturity of PCs. The concentration of the medium-sized PL-EVs (180 to $300 \mathrm{~nm}$ ) did not match the PL-EV ratio of expired PCs in significance of correlation to the standard system. This can be explained by the fact that during storage of platelets the more small formed vesicles are underestimated and, thus, do not influence the PL-EV ratio.

The notion can be confirmed by an experience in application of the NTA methods to measurement of "old samples from PCs" characterized by an enormous increase of very small particles which escaped the analysis in the current dilution protocol. Although the plasma vesicles of the expired PCs were in average larger as measured by NTA, more peaks of vesicle smaller than $100 \mathrm{~nm}$ were recognized. The analysis by NTA does not show any correlation to a completely different method of flow cytometry at any step of the investigation. The method is for a certainty well suited for the estimation of all vesicles in PC samples, even for the plasma samples comprising high amounts of lipid vesicles and protein aggregates, and it provides a tool to determine the size of particles or vesicles in the samples. Recent investigations showed vesicle determination in PCs with a similar method, dynamic light scattering with ThromboLUX®, to define good quality of products (291). Labrie et al. involved microparticles in a scoring system according to platelet increment after application of PCs and concluded that all microparticles have to be excluded from PCs to guarantee sufficient product quality. In order to different origins of vesicles (i.e. proteins, cells, lipids, immuncomplexes) it was not examined, if separated vesicles may provide additional transfusion related functions. Nevertheless, without any categorical labeling of platelet-derived or membrane-derived vesicles, the specificity of the NTA method in this study is not congruent with the requirements of $Q C$ analysis of cell components or any other pharmaceutical product.

Taking into consideration the fact that hs-FCM may be applied as a possible method of PL-EV analysis in PC control, it has to be addressed, whether the PL-EV ratio or the PL-EV concentration are reliably coherent to a gold standard of QC analysis. Without exception, the PL-EV levels measured by hs-FCM, negatively correlate to the expression of CD62P after its activation with TRAP-6. The method is suitable as a predictive test of the quality of platelets derived from both fresh and expired PCs. Therefore, it is necessary to continue investigation elucidating the fact whether the PL-EV ratio, the concentration of medium, larger or all PL-EVs 
are the most effective predictors. To sum up, the new FCM system can be accepted as a future method of choice in vesicle analysis in QC program of PCs. The method could be fast and easily implemented in routine QC tests in parallel to gold standard platelet function analysis. It is more useful than sd-FCM to detect the relationship between former activation of platelets/ loss of responsiveness and their vesicle secretion.

As mentioned above, PL-EVs or membrane-derived vesicles may constitute the most abundant components in the composition of all vesicles in human body fluids (248) and, as far as this study is concerned in PCs. As the results of the present research and further investigations of cell-derived vesicles from PCs demonstrate, no contamination with leukocyte-derived or red blood cell-derived vesicles was found (146).

More than 95 percent of all PC vesicles detected with the sd-FCM method originated from platelets. The storage of platelets and the vesiculation of residual erythrocytes or leukocytes in PCs over time do not affect the aforementioned amount. It is speculated that leukocyte-derived vesicles in PC samples are hardly detectable and, consequently, underestimated by sd-FCM analysis. Additional researches are of importance in order to improve the identification of residual vesicles of remaining external cells. However, in the present study these vesicles were not in focus of interest as a component of vesicle population of PCs.

In contrast, parallel tests on red blood cell (RBC) units which were analyzed in terms of vesicle composition, showed enormous contamination with foreign membrane vesicles, i.e. plateletderived. In undiluted RBC units, approx. 20 percent of PL-EVs were detected by sd-FCM. One can question these results and criticize inadequate pre-analytical preparation of the samples from RBC units exhibiting too high concentration of RBCs for a valid measurement by flow cytometry. However, the more RBC units were diluted, the more percent of PL-EV were obtained. Interestingly, the preparation of exosome fraction by ultra-centrifugation showed, particularly in the third pellet after preparation, cell-derived vesicles originated from other celltypes primary not included in the concentrates. This aspect needs to be further investigated. However, it needs urgent research whether PL-EVs derived from RBC units are useful as procoagulant vesicles transferred during the application of RBC units to anemia patients or are carriers of specific proteins that affect both, red blood cells in concentrates and patient response after transfusion, thus causing adverse effects,

Despite of the fact that every milliliter of concentrate gained in apheresis is extremely valuable, further investigations are necessary with the purpose of determination of extracellular vesicles in concentrates of peripheral blood stem cell apheresis.

It is true that some cell-derived vesicles, for example, erythrocyte-derived vesicles (47), may be less influenced by washing steps or activation during centrifugation and they could be interpreted as false positive contamination of the blood concentrate of foreign cell type lineage; yet, the fact remains that type-specific vesicles are not the only structures found in the appropriate concentrates. They could appear due to the cell stress during apheresis as well as 
due to other preparation techniques and they may be implemented in the plasma components of apheresis concentrates. Otherwise, the residual erythrocytes and residual leukocytes are sufficient to form MVE (multivesicular endosomes) and their secretion or degradation might be the only longer living breath of cells in vitro.

On the whole, findings of EVs in blood components raise an important clinical question in context of the application of blood products to patients, namely, whether activation of PLTs and procoagulant PL-EVs in PCs are more effective in general or applicable to a selective group of patients only. For example, patients with severe hematologic-oncologic diseases need concentrates with less activated and less shedded PLTs for prophylactic treatment. These platelets may exhibit longer survival and viability in circulation and are not subjected to rapid clearance. In contrast, patients with polytraumata settings, with sepsis or MODS may benefit from more coagulant PCs consisting of differentially affected PLTs. The application of various types of vesicles may influence the clinical treatment as there is a choice between fresh PCs and altered PLTs/ PL-EVs.

Finally, it has to be kept in mind that PCs comprise different amount of PL-EVs. PL-EVs range in size between approx. $70 \mathrm{~nm}$ and $550 \mathrm{~nm}$. Moreover, considering the results of this study, it would be of importance to elucidate what kind of platelet activatability is to be expected after five days of storage. Such information could be obtained through a simple, applicable analysis of PL-EV amount in concentrates.

In conclusion, many different aspects affecting the activation of PLTs from PCs and, consequently, EV production were presented and discussed in this dissertation. The amount of PL-EVs in PCs may be influenced by numerous factors such as donor-specific variables, the course of the manufacturing process and storage, pre-analytical factors and level of responsiveness of PLTs. Thus, it is crucial to gather more information on PL-EVs concerning their phenotype, formation process, proteomics and functional genomics in order to unravel mechanisms of coagulation of PL-EVs in PCs. The same information should be provided in order to transfusion that is more successful. 


\section{Summary}

Platelet-derived extracellular vesicles (PL-EVs) constitute the major part of circulating EVs in blood. They are also present in platelet concentrates (PCs) and may influence the quality of PCs as recent reports implicate significant pro-thrombotic and pro-coagulant activities of PLEVs. The aim of the present study was to analyze PC-derived PL-EVs and to correlate them with standard quality control (QC) parameters of PCs (e.g. functional capacity of platelets, duration of hemapheresis) and with donor-specific laboratory parameters (e.g. BMI, immature platelet fraction).

PL-EVs, shed from activated and senescent platelets, were analyzed by nanoparticle tracking analysis (NTA) and standard (Navios ${ }^{\mathrm{TM}}$ ) as well as by advanced high sensitivity (Apogee $50 \mathrm{M}$ ) flow cytometry. A hematology analyzer was applied to the determination of the platelet count and immature platelet fraction (IPF). Functional capacity of platelets (i.e. externalization of CD62P in response to standard TRAP-6 activation) was measured by flow cytometry. Standard QC parameters were recorded during platelet hemapheresis. All in vitro measurements were carried out on day 0 (PC-production, 'fresh platelets') and on day 5 (after PC expiry, 'senescent platelets'). Altogether, $n=106 \mathrm{PC}$-samples were investigated and $\mathrm{n}=42$ (15 of them were irradiated with 25 Gy on day 0 ) were included in the specific statistical analysis.

The externalization of CD62P, indicative for intact platelet function in PCs, significantly decreased during in vitro platelet senescence and this was inversely correlated with the significant increase of PL-EVs concentration. The size of PL-EVs varied in all PCs investigated, ranging from approx. $70 \mathrm{~nm}$ to $550 \mathrm{~nm}$, suggesting that the size spectrum of PL-EVs depends on different vesicle formation mechanisms based on corresponding PLT condition.

Interestingly, in fresh PCs it was found that there is a significant correlation between PL-EVs and PC-production with different hemapheresis instruments, duration of platelet apheresis and the IPF count in peripheral blood of the donor prior to apheresis. In senescent PCs, the BMI of donors inversely correlated with the PL-EV counts.

Accurate measurement of PL-EVs is highly recommended in the regular QC of PCs as a plausibility check of platelet function. Shedding of PL-EVs in PCs depends on shear stress in hemapheresis and diverse pre-analytical conditions, leading to a conclusion that the quality of platelet concentrates is strongly influenced by PL-EVs. 


\section{Zusammenfassung}

Im zirkulierenden Blut des Menschen stammt der überwiegende Anteil der extrazellulären Vesikel (EVs) von Thrombozyten und Megakaryozyten ab. Die thrombozytären EVs kommen ebenfalls in Apheresekonzentraten, wie beispielsweise Thrombozytapheresekonzentraten (TKs) vor und beeinflussen die Qualität der Blutprodukte auf unterschiedliche, noch nicht umfassend geklärte Weise. Unter anderem haben thrombozytäre EVs erwiesenermaßen pro-thrombotische und pro-koagulatorische Eigenschaften, die damit auch die pharmazeutische Wirkung der Blutprodukte beeinflussen.

Ziel der vorliegenden Studie war, den qualitativen und quantitativen Nachweis von thrombozytären EVs in TKs mit den aktuell standardisierten Parametern der Qualitätskontrolle (QK) zu korrelieren. Standardtests umfassten dabei unter anderem die funktionelle Kapazität von Thrombozyten im TK und die Apheresedauer bei Herstellung der TKs und die damit verbundene Aktivierung der Plättchen durch Scherkräfte. Es wurden ebenfalls Spenderspezifische Laborparameter, wie Glukose, Thrombozytenzahl vor Apherese oder unreife Thrombozytenfraktion, als auch klinisch orientierende Parameter (z.B. Body Mass Index, BMI) auf einen Zusammenhang mit dem Nachweis von thrombozytären EVs hin untersucht.

Thrombozytäre EVs werden von der Membran aktivierter und/ oder alternder Plättchen abgeschnürt. Diese wurden mittels eines Nanoparticle Tracking Analysis (NTA)-Verfahrens, eines Standard Durchflusszytometers (Navios ${ }^{\mathrm{TM}}$ ), als auch mit einem hochauflösenden Durchflusszytometer (Apogee 50M) gemessen. Die Thrombozytenzahl und Fraktion unreifer Thrombozyten wurde mit einem automatisierten Hämatologie-Automaten bestimmt. Die funktionelle Kapazität von Thrombozyten anhand der Expression von P-Selectin (CD62P) nach Aktivierung mit TRAP-6-Reagenz wurde durchflusszytometrisch erfasst. Die übrigen StandardParameter für die QK wurden während der Aphereseprozedur ermittelt. Alle in vitro Tests wurden an frischen Thrombozyten am Tag der Herstellung ( $\operatorname{Tag} 0)$ und mit gealterten Thrombozyten bei sachgerechter Lagerung der Konzentrate nach Ablauf der Haltbarkeit (Tag 5) durchgeführt. Insgesamt wurden 106 TK-Proben untersucht. Von allen Proben wurden 42 TKs, darunter 15 bestrahlte TKs (25 Gy am Tag der Herstellung), in die spezielle Auswertung eingeschlossen.

Die Expression von CD62P als Indikator für intakte, funktionsfähige Thrombozyten, nahm während der in vitro Lagerung der TKs über einen Zeitraum von 5 Tagen signifikant ab. Diese Funktionsabnahme der Plättchen korrelierte signifikant invers mit der Zunahme von thrombozytären EVs pro Thrombozytenzahl. Die Größe der thrombozytären EVs der untersuchten TKs variierte heterogen in einem Bereich von $70-550 \mathrm{~nm}$, was darauf schließen lässt, dass verschiedene Mechanismen der Produktion von EVs zu Grunde liegen. Interessanterweise zeigten sich signifikante Unterschiede des EV-Gehaltes in frischen TKs durch den Einsatz unterschiedlicher Apherese-Maschinen, durch die unterschiedliche 
Apheresedauer und die Fraktion unreifer Thrombozyten aus peripherem Blut der Spender vor der Apherese. In TKs mit gealterten Thrombozyten korrelierte der Anteil thrombozytärer EVs invers mit dem BMI der Spender.

Die Messung des Anteils an thrombozytären EVs in TKs ist als sinnvoller Plausibilitätstests zur Funktionalität der Thrombozyten in der regulären QK zu empfehlen. Die Vesikulation von thrombozytären EVs in TKs ist abhängig vom Scherstress der Thrombozyten während der Herstellung und ist abhängig von verschiedenen anderen präanalytischen und Spenderspezifischen Faktoren. Demzufolge beeinflusst der Anteil an thrombozytären EVs die Qualität von Thrombozytapheresekonzentraten. 


\section{Publications}

Matysik S, Orsó E, Black A, Ahrens N, Schmitz G.; Monitoring of 7a-hydroxy-4-cholesten-3-one during therapy of cerebrotendinous xanthomatosis: a case report. Chem Phys Lipids. 2011 Sep; 164(6):530-4

Black A., Mack M., Dullinger K., Schmitz G., Ahrens N.; Isoagglutinin apheresis for ABOincompatible renal Transplantation. Transfus Med Hemother 2011;38(suppl 1):1-72; Abstract

Black A., Ditz D., Holler E., Schmitz G., Ahrens N.; Are antibodies to the blood group antigen M clinically relevant? Transfus Med Hemother 2011;38(suppl 1):1-72; Abstract

Black A., Pienimäki-Römer A., Orsó, E., Schmitz, G.; Platelet-derived extracellular vesicles influence the quality of platelet concentrates. Transfus Med Hemother 2013; Abstract

Pienimaeki Roemer, A. ", Katja Kuhlmann, K. ${ }^{*}$, Böttcher, A., Konovalova, T., Black, A., Orsó, E., Liebisch, G., Ahrens, M., Eisenacher, M., Meyer, H.E., Schmitz, G.; *contributed equally to the manuscript; Lipidomic and proteomic characterization of platelet extracellular vesicle subfractions from senescent platelets; Trans-2014-0279.R2, in press

Black A., Pienimäki-Römer A., Kenyon, O., Orsó, E., Schmitz, G.; Platelet-derived extracellular vesicles in plateletpheresis concentrates as a quality control approach, Trans-2014-0351.R1, Revised manuscript in Draft 


\section{Curriculum vitae}

\section{Professional experiences}

$03 / 2010-03 / 2012$

University Hospital Regensburg, (Germany)

Department: Clinical chemistry, laboratory medicine and transfusion medicine

- Assistant physician (Transfusion medicine)

since $04 / 2012$

University Hospital Regensburg, (Germany)

Department: Clinical chemistry, laboratory medicine and transfusion medicine

- Assistant physician (Laboratory medicine)

$10 / 2008-01 / 2009$

St. Hedwig, Barmherzige Brüder Regensburg (Germany)

Department: Pediatric hematology and oncology

- Practical year of education

06/2008 - 10/2008 Klinikum Passau, (Germany)

Department: Thoracic and visceral surgery

- Practical year of education

$02 / 2008-06 / 2008$

Klinikum Passau, (Germany)

Department: Internal medicine

- Practical year of education

$\underline{\text { Academic career }}$

10/2002 - 08/2009 University of Regensburg (Germany)

Study of medicine

09/2004-07/2007 Marie-Curie-Gymnasium, Wittenberge (Germany)

Higher education entrance qualification 
Extra-curricular activities

08. - 10.03.2013 Advanced hematological cytology workshop (Eschweiler, Germany)

02/2012 Attend classes as a guest physician in Immunohematology and Clinical Diagnostics, Prof. Salama, Charité Berlin (Germany)

06/2010 Lipid-Apheresis User-Meeting, Charité Berlin (Germany)

27. $-30.10 .2009 \quad$ Bone marrow cytology workshop, Leipzig (Germany)

08/2009-11/2009 Scientific assistant at Department of Pediatric hematology and oncology, St. Hedwig, Barmherzige Brüder Regensburg (Germany)

12/2005 - 12/2007 Practical assistant of General Practitioner Dr. V. Schnell, Regensburg

10/2003 - 10/2004 Student assistant at the Institute of anatomy, University of Regensburg 


\section{Acknowledgements}

Ich bedanke mich besonders bei Prof. Dr. Gerd Schmitz für die Möglichkeit der Durchführung meiner medizinischen Dissertation am Institut für Klinische Chemie und Laboratoriumsmedizin mit den nötigen technischen Voraussetzungen. Die Umsetzung der Arbeit wurde unmittelbar durch die Genehmigung zur Fortführung meiner Facharztausbildung als Laboratoriumsmedizinerin an diesem Hause unterstützt, die er im Wesentlichen beeinflusst hat.

Ganz besonders bedanken möchte ich mich bei Dr. Evelyn Orsó, die mit Ihrer fachlichen Kompetenz jederzeit für Fragen zur Verfügung stand und dazu beigetragen hat, das Thema der Arbeit zu verifizieren. Insbesondere ist im durchflusszytometrischen Arbeitsbereich mein Interesse am Verfahren und dessen analytischen Möglichkeiten durch ihre konstante und unermüdliche Bereitschaft der Wissensvermittlung gewachsen. Ihre Kompetenz in diesem Bereich ist in großem Umfang in die Durchführung der gesamten Arbeit mit eingeflossen.

Vielen weiteren Mitarbeitern unterschiedlicher Arbeitsbereiche des Instituts gebührt ebenfalls mein Dank. Zu erwähnen ist, neben der Bereitschaft der Spender, die qualitativ hochwertige und freundliche Arbeit des Personals der Transfusionsmedizin bei der Herstellung der Thrombozytapheresekonzentrate und die Arbeit der Leitung der Qualitätskontrolle. Ebenfalls danke ich den medizinisch technischen Angestellten für die präzise und kompetente Bearbeitung der Spender- und TK-Proben an den automatisierten Geräten des Labors im Bereich der klinischen Chemie, Rheumatologie, Endokrinologie, Toxikologie, der zellulären Diagnostik und der Blutbank, sowie auch der Probenannahme.

Bei der Erarbeitung der Methode zur Messung der EVs aus Erythrozytenkonzentraten konnte ich auf die langjährige und fundierte Erfahrung von Dr. Alfred Böttcher zurückgreifen und bedanke mich bei inm und Renate Kick für die Hilfe bei der Präparation der EKs.

Für die Hilfe bei den Messungen am Apogee und bei den Aggregometrie-Messungen danke ich herzlich Dr. Annika Piennimaeki-Römer. Ich danke Florian Zeman, Zentrum für klinische Studien, für die Hilfestellung zur statistischen Auswertung. Ebenfalls bedanke ich mich ganz herzlich bei Dr. Anna Schmitz-Madry für die Beratung zur Verbesserung des sprachlichen Stils dieser Arbeit.

Moralische Unterstützung und Motivation verdanke ich herzlichst meinen Arbeitskollegen und Arbeitskolleginnen Dr. Susanne Heimerl, Dr. Silke Matysik, Jana Kirsten, Sabine Sattler, Dr. Thomas Kopf, PD Dr. Gerhard Liebisch, Dr. Alexander Sigrüner und Dr. Stefan Wallner.

Meine Arbeit ist im Besonderen begleitet worden von der unendlichen Unterstützung meiner Familie. Ich danke meiner mutigen Tochter, meinem geduldigen Lebenspartner, meinen Eltern und Geschwistern, die mir nicht nur Verständnis entgegengebracht, sondern auch immer das Vertrauen an mich selbst bewahrt haben. 


\section{Eidesstattliche Erklärung}

Ich erkläre, dass ich den Doktorgrad der Medizin nicht schon an einer Hochschule der Bundesrepublik Deutschland erworben habe. Ebenfalls bin ich nicht an anderer Stelle zu einem Promotionsverfahren zum Erwerb des medizinischen Doktorgrades zugelassen. Ferner habe ich nicht schon eine medizinische Doktorprüfung an einer Hochschule der Bundesrepublik Deutschland endgültig bestanden.

Außerdem erkläre ich, dass mir keine Tatsachen bekannt sind, die mich zur Führung eines akademischen Grades im Sinne des Gesetzes über die Führung akademischer Grade unwürdig erscheinen lassen. Ich versichere, dass ich die vorliegende Arbeit ohne unzulässige Hilfe Dritter und ohne Benutzung anderer als der angegebenen Hilfsmittel angefertigt habe. Die aus anderen Quellen direkt oder indirekt übernommenen Daten und Konzepte sind unter Angabe der Quelle gekennzeichnet. Insbesondere habe ich nicht die entgeltliche Hilfe von Vermittlungsbzw. Beratungsdiensten (Promotionsberater oder andere Personen) in Anspruch genommen. Niemand hat von mir unmittelbar oder mittelbar geldwerte Leistungen für Arbeit erhalten, die im Zusammenhang mit dem Inhalt der vorgelegten Dissertation stehen. Die Arbeit wurde bisher weder im In- noch im Ausland in gleicher oder ähnlicher Form einer anderen Prüfungsbehörde vorgelegt. 


\section{References}

(1) Hartley PS. Platelet senescence and death. Clin Lab 2007;53(3-4):157-66.

(2) Josefsson EC, Dowling MR, Lebios M, Kile BT. The regulation of platelet life span. 3rd ed. 2012. p. 51.

(3) Flaumenhaft R. Platelet secretion. 3rd ed. 2013. p. 343.

(4) Ohto $\mathrm{H}$, Nollet KE. Overview on platelet preservation: better controls over storage lesion. Transfus Apher Sci 2011 Jun;44(3):321-5.

(5) Perrotta PL, Parsons J, Rinder HM, Snyder EL. Platelet transfusion medicine. 3rd ed. 2012. p. 1275.

(6) Badlou BA, ljseldijk MJ, Smid WM, Akkerman JW. Prolonged platelet preservation by transient metabolic suppression. Transfusion 2005 Feb;45(2):214-22.

(7) George JN, Pickett EB, Heinz R. Platelet membrane glycoprotein changes during the preparation and storage of platelet concentrates. Transfusion 1988 Mar;28(2):123-6.

(8) Wolf $\mathrm{P}$. The nature and significance of platelet products in human plasma. $\mathrm{Br} \mathrm{J}$ Haematol 1967 May;13(3):269-88.

(9) Nieuwland R, van der Pol E, Gardiner C, Sturk A. Platelet-derived microparticles. 3rd ed. 2012. p. 453.

(10) Bode AP, Miller DT. Analysis of platelet factor 3 in platelet concentrates stored for transfusion. Vox Sang 1986;51(4):299-305.

(11) Flaumenhaft R. Formation and fate of platelet microparticles. Blood Cells Mol Dis $2006 \operatorname{Mar} ; 36(2): 182-7$.

(12) Solberg C, Holme S, Little C. Morphological changes associated with $\mathrm{pH}$ changes during storage of platelet concentrates. Beitr Infusionther Klin Ernahr 1986;15:107-17.

(13) Solberg C, Osterud B, Little C. Platelet storage lesion: formation of platelet fragments with platelet factor 3 activity. Thromb Res 1987 Dec 1;48(5):559-65.

(14) Bode AP, Orton SM, Frye MJ, Udis BJ. Vesiculation of platelets during in vitro aging. Blood 1991 Feb 15;77(4):887-95.

(15) Rank A, Nieuwland R, Liebhardt S, Iberer M, Grutzner S, Toth B, et al. Apheresis platelet concentrates contain platelet-derived and endothelial cell-derived microparticles. Vox Sang 2011 Feb;100(2):179-86.

(16) Sloand EM, Yu M, Klein HG. Comparison of random-donor platelet concentrates prepared from whole blood units and platelets prepared from single-donor apheresis collections. Transfusion $1996 \mathrm{Nov;36(11-12):955-9.}$

(17) Seghatchian J, Krailadsiri P. The platelet storage lesion. Transfus Med Rev 1997 Apr;11(2):130-44.

(18) Leytin V, Freedman J. Platelet apoptosis in stored platelet concentrates and other models. Transfus Apher Sci 2003 Jun;28(3):285-95. 
(19) Bertino AM, Qi XQ, Li J, Xia Y, Kuter DJ. Apoptotic markers are increased in platelets stored at 37 degrees C. Transfusion 2003 Jul;43(7):857-66.

(20) Thiele T, Steil L, Gebhard S, Scharf C, Hammer E, Brigulla M, et al. Profiling of alterations in platelet proteins during storage of platelet concentrates. Transfusion 2007 Jul;47(7):1221-33.

(21) Thiele T, luga C, Janetzky S, Schwertz H, Gesell SM, Furll B, et al. Early storage lesions in apheresis platelets are induced by the activation of the integrin alphallbbeta(3) and focal adhesion signaling pathways. J Proteomics 2012 Dec 5;76 Spec No.:297-315.

(22) Schubert P, Thon JN, Walsh GM, Chen CH, Moore ED, Devine DV, et al. A signaling pathway contributing to platelet storage lesion development: targeting PI3-kinasedependent Rap1 activation slows storage-induced platelet deterioration. Transfusion 2009 Sep;49(9):1944-55.

(23) Perrotta PL, Perrotta CL, Snyder EL. Apoptotic activity in stored human platelets. Transfusion 2003 Apr;43(4):526-35.

(24) Albanyan AM, Harrison P, Murphy MF. Markers of platelet activation and apoptosis during storage of apheresis- and buffy coat-derived platelet concentrates for 7 days. Transfusion 2009 Jan;49(1):108-17.

(25) Albanyan A, Murphy MF, Wilcock M, Harrison P. Changes in the immature platelet fraction within ageing platelet concentrates. J Thromb Haemost 2008 Dec;6(12):22135.

(26) Hunter MP, Ismail N, Zhang X, Aguda BD, Lee EJ, Yu L, et al. Detection of microRNA expression in human peripheral blood microvesicles. PLoS One 2008;3(11):e3694.

(27) Valadi H, Ekstrom K, Bossios A, Sjostrand M, Lee JJ, Lotvall JO. Exosome-mediated transfer of mRNAs and microRNAs is a novel mechanism of genetic exchange between cells. Nat Cell Biol 2007 Jun;9(6):654-9.

(28) Collino F, Deregibus MC, Bruno S, Sterpone L, Aghemo G, Viltono L, et al. Microvesicles derived from adult human bone marrow and tissue specific mesenchymal stem cells shuttle selected pattern of miRNAs. PLoS One 2010;5(7):e11803.

(29) Tynngard N, Studer M, Lindahl TL, Trinks M, Berlin G. The effect of gamma irradiation on the quality of apheresis platelets during storage for 7 days. Transfusion 2008 Aug;48(8):1669-75.

(30) Thiele T, Sablewski A, luga C, Bakchoul T, Bente A, Gorg S, et al. Profiling alterations in platelets induced by Amotosalen/UVA pathogen reduction and gamma irradiation-a LC-ESI-MS/MS-based proteomics approach. Blood Transfus 2012 May;10 Suppl 2:s63-s70.

(31) American Association of Blood Banks. AABB's Technical Manual. 17th ed. 2011.

(32) Guide to the preparation, use and quality assurance of blood components: Recommendation No. R(95) 15. 14th ed. Council of Europe, 2008.

(33) Bundesärztekammer, Paul-Ehrlich-Institut. Richtlinien zur Gewinnung von Blut und Blutbestandteilen und zur Anwendung von Blutprodukten (Hämotherapie). 2010. 
(34) Murphy S. Radiolabeling of PLTs to assess viability: a proposal for a standard. Transfusion 2004 Jan;44(1):131-3.

(35) Bertolini F, Agazzi A, Peccatori F, Martinelli G, Sandri MT. The absence of swirling in platelet concentrates is highly predictive of poor posttransfusion platelet count increments and increased risk of a transfusion reaction. Transfusion 2000 Jan;40(1):121-2.

(36) Divers SG, Kannan K, Stewart RM, Betzing KW, Dempsey D, Fukuda M, et al. Quantitation of CD62, soluble CD62, and lysosome-associated membrane proteins 1 and 2 for evaluation of the quality of stored platelet concentrates. Transfusion 1995 Apr;35(4):292-7.

(37) Holme S, Sweeney JD, Sawyer S, Elfath MD. The expression of p-selectin during collection, processing, and storage of platelet concentrates: relationship to loss of in vivo viability. Transfusion 1997 Jan;37(1):12-7.

(38) Berger G, Hartwell DW, Wagner DD. P-Selectin and platelet clearance. Blood 1998 Dec 1;92(11):4446-52.

(39) Michelson AD, Barnard MR, Hechtman HB, MacGregor H, Connolly RJ, Loscalzo J, et al. In vivo tracking of platelets: circulating degranulated platelets rapidly lose surface P-selectin but continue to circulate and function. Proc Natl Acad Sci U S A 1996 Oct 15;93(21):11877-82.

(40) Caby MP, Lankar D, Vincendeau-Scherrer C, Raposo G, Bonnerot C. Exosomal-like vesicles are present in human blood plasma. Int Immunol 2005 Jul;17(7):879-87.

(41) Taylor DD, Akyol S, Gercel-Taylor C. Pregnancy-associated exosomes and their modulation of T cell signaling. J Immunol 2006 Feb 1;176(3):1534-42.

(42) Admyre C, Johansson SM, Qazi KR, Filen JJ, Lahesmaa R, Norman M, et al. Exosomes with immune modulatory features are present in human breast milk. $\mathrm{J}$ Immunol 2007 Aug 1;179(3):1969-78.

(43) Asea A, Jean-Pierre C, Kaur P, Rao P, Linhares IM, Skupski D, et al. Heat shock protein-containing exosomes in mid-trimester amniotic fluids. J Reprod Immunol 2008 Oct;79(1):12-7.

(44) Pisitkun T, Shen RF, Knepper MA. Identification and proteomic profiling of exosomes in human urine. Proc Natl Acad Sci U S A 2004 Sep 7;101(36):13368-73.

(45) Nieuwland R, Sturk A. Why do cells release vesicles? Thromb Res 2010 Apr;125 Suppl 1:S49-S51.

(46) Théry $C$, Ostrowski $M$, Segura E. Membrane vesicles as conveyors of immune responses. Nat Rev Immunol 2009 Aug;9(8):581-93.

(47) van der Pol E, Boing AN, Harrison P, Sturk A, Nieuwland R. Classification, functions, and clinical relevance of extracellular vesicles. Pharmacol Rev 2012 Jul;64(3):676705.

(48) Gould SJ, Raposo G. As we wait: coping with an imperfect nomenclature for extracellular vesicles. Journal of Extracellular Vesicles; Vol 2 (2013) 2013 Feb 15. 
(49) van der Pol E, van Gemert MJ, Sturk A, Nieuwland R, van Leeuwen TG. Single vs. swarm detection of microparticles and exosomes by flow cytometry. J Thromb Haemost 2012 May;10(5):919-30.

(50) Webber J, Clayton A. How pure are your vesicles? Journal of Extracellular Vesicles; Vol 2 (2013) 2013 Jan 14.

(51) Théry C, Boussac M, Veron P, Ricciardi-Castagnoli P, Raposo G, Garin J, et al. Proteomic analysis of dendritic cell-derived exosomes: a secreted subcellular compartment distinct from apoptotic vesicles. J Immunol 2001 Jun 15;166(12):730918.

(52) Akers JC, Gonda D, Kim R, Carter BS, Chen CC. Biogenesis of extracellular vesicles $(\mathrm{EV})$ : exosomes, microvesicles, retrovirus-like vesicles, and apoptotic bodies. J Neurooncol 2013 Mar 2.

(53) Raposo G, Nijman HW, Stoorvogel W, Liejendekker R, Harding CV, Melief CJ, et al. B lymphocytes secrete antigen-presenting vesicles. J Exp Med 1996 Mar 1;183(3):1161-72.

(54) Heijnen HF, Schiel AE, Fijnheer R, Geuze HJ, Sixma JJ. Activated platelets release two types of membrane vesicles: microvesicles by surface shedding and exosomes derived from exocytosis of multivesicular bodies and alpha-granules. Blood 1999 Dec 1;94(11):3791-9.

(55) Cocucci E, Racchetti G, Meldolesi J. Shedding microvesicles: artefacts no more. Trends Cell Biol 2009 Feb;19(2):43-51.

(56) Orozco AF, Lewis DE. Flow cytometric analysis of circulating microparticles in plasma. Cytometry A 2010 Jun;77(6):502-14.

(57) Elmore S. Apoptosis: a review of programmed cell death. Toxicol Pathol 2007 Jun;35(4):495-516.

(58) Hristov M, Erl W, Linder S, Weber PC. Apoptotic bodies from endothelial cells enhance the number and initiate the differentiation of human endothelial progenitor cells in vitro. Blood 2004 Nov 1;104(9):2761-6.

(59) Ihara $T$, Yamamoto $T$, Sugamata $M$, Okumura $H$, Ueno $Y$. The process of ultrastructural changes from nuclei to apoptotic body. Virchows Arch 1998 Nov;433(5):443-7.

(60) del Conde I, Shrimpton CN, Thiagarajan P, Lopez JA. Tissue-factor-bearing microvesicles arise from lipid rafts and fuse with activated platelets to initiate coagulation. Blood 2005 Sep 1;106(5):1604-11.

(61) Klibi J, Niki T, Riedel A, Pioche-Durieu C, Souquere S, Rubinstein E, et al. Blood diffusion and Th1-suppressive effects of galectin-9-containing exosomes released by Epstein-Barr virus-infected nasopharyngeal carcinoma cells. Blood 2009 Feb 26;113(9):1957-66.

(62) Sprague DL, Elzey BD, Crist SA, Waldschmidt TJ, Jensen RJ, Ratliff TL. Plateletmediated modulation of adaptive immunity: unique delivery of CD154 signal by platelet-derived membrane vesicles. Blood 2008 May 15;111(10):5028-36. 
(63) Skog J, Wurdinger T, van RS, Meijer DH, Gainche L, Sena-Esteves M, et al. Glioblastoma microvesicles transport RNA and proteins that promote tumour growth and provide diagnostic biomarkers. Nat Cell Biol 2008 Dec;10(12):1470-6.

(64) Hong BS, Cho JH, Kim H, Choi EJ, Rho S, Kim J, et al. Colorectal cancer cell-derived microvesicles are enriched in cell cycle-related mRNAs that promote proliferation of endothelial cells. BMC Genomics 2009;10:556.

(65) Mittelbrunn M, Gutierrez-Vazquez C, Villarroya-Beltri C, Gonzalez S, Sanchez-Cabo $\mathrm{F}$, Gonzalez MA, et al. Unidirectional transfer of microRNA-loaded exosomes from T cells to antigen-presenting cells. Nat Commun 2011;2:282.

(66) Montecalvo A, Larregina AT, Shufesky WJ, Stolz DB, Sullivan ML, Karlsson JM, et al. Mechanism of transfer of functional microRNAs between mouse dendritic cells via exosomes. Blood 2012 Jan 19;119(3):756-66.

(67) Pegtel DM, Cosmopoulos K, Thorley-Lawson DA, van Eijndhoven MA, Hopmans ES, Lindenberg JL, et al. Functional delivery of viral miRNAs via exosomes. Proc Natl Acad Sci U S A 2010 Apr 6;107(14):6328-33.

(68) Lässer C, Alikhani VS, Ekstrom K, Eldh M, Paredes PT, Bossios A, et al. Human saliva, plasma and breast milk exosomes contain RNA: uptake by macrophages. J Transl Med 2011;9:9.

(69) Sims PJ, Faioni EM, Wiedmer T, Shattil SJ. Complement proteins C5b-9 cause release of membrane vesicles from the platelet surface that are enriched in the membrane receptor for coagulation factor $\mathrm{Va}$ and express prothrombinase activity. $\mathrm{J}$ Biol Chem 1988 Dec 5;263(34):18205-12.

(70) Shedden K, Xie XT, Chandaroy P, Chang YT, Rosania GR. Expulsion of small molecules in vesicles shed by cancer cells: association with gene expression and chemosensitivity profiles. Cancer Res 2003 Aug 1;63(15):4331-7.

(71) Abid Hussein MN, Boing AN, Sturk A, Hau CM, Nieuwland R. Inhibition of microparticle release triggers endothelial cell apoptosis and detachment. Thromb Haemost 2007 Nov;98(5):1096-107.

(72) de Gassart A., Geminard C, Fevrier B, Raposo G, Vidal M. Lipid raft-associated protein sorting in exosomes. Blood 2003 Dec 15;102(13):4336-44.

(73) Merten M, Pakala R, Thiagarajan P, Benedict CR. Platelet microparticles promote platelet interaction with subendothelial matrix in a glycoprotein Ilb/Illa-dependent mechanism. Circulation 1999 May 18;99(19):2577-82.

(74) Clayton A, Turkes A, Dewitt S, Steadman R, Mason MD, Hallett MB. Adhesion and signaling by $B$ cell-derived exosomes: the role of integrins. FASEB J 2004 Jun;18(9):977-9.

(75) Sirois I, Raymond MA, Brassard N, Cailhier JF, Fedjaev M, Hamelin K, et al. Caspase-3-dependent export of TCTP: a novel pathway for antiapoptotic intercellular communication. Cell Death Differ 2011 Mar;18(3):549-62.

(76) Castaman G, Yu-Feng L, Battistin E, Rodeghiero F. Characterization of a novel bleeding disorder with isolated prolonged bleeding time and deficiency of platelet microvesicle generation. Br J Haematol 1997 Mar;96(3):458-63. 
(77) Castaman G, Yu-Feng L, Rodeghiero F. A bleeding disorder characterised by isolated deficiency of platelet microvesicle generation. Lancet 1996 Mar 9;347(9002):700-1.

(78) Morel O, Jesel L, Freyssinet JM, Toti F. Cellular mechanisms underlying the formation of circulating microparticles. Arterioscler Thromb Vasc Biol 2011 Jan;31(1):15-26.

(79) Owens AP, III, Mackman N. Microparticles in hemostasis and thrombosis. Circ Res 2011 May 13;108(10):1284-97.

(80) Perez-Pujol S, Marker PH, Key NS. Platelet microparticles are heterogeneous and highly dependent on the activation mechanism: studies using a new digital flow cytometer. Cytometry A 2007 Jan;71(1):38-45.

(81) Connor DE, Exner T, Ma DD, Joseph JE. The majority of circulating platelet-derived microparticles fail to bind annexin V, lack phospholipid-dependent procoagulant activity and demonstrate greater expression of glycoprotein Ib. Thromb Haemost 2010 May;103(5):1044-52.

(82) Joop K, Berckmans RJ, Nieuwland R, Berkhout J, Romijn FP, Hack CE, et al. Microparticles from patients with multiple organ dysfunction syndrome and sepsis support coagulation through multiple mechanisms. Thromb Haemost 2001 May;85(5):810-20.

(83) Broze GJ, Jr. Tissue factor pathway inhibitor. Thromb Haemost 1995 Jul;74(1):90-3.

(84) Mackman N, Tilley RE, Key NS. Role of the extrinsic pathway of blood coagulation in hemostasis and thrombosis. Arterioscler Thromb Vasc Biol 2007 Aug;27(8):1687-93.

(85) Berckmans RJ, Sturk A, van Tienen LM, Schaap MC, Nieuwland R. Cell-derived vesicles exposing coagulant tissue factor in saliva. Blood 2011 Mar 17;117(11):317280.

(86) Berckmans RJ, Nieuwland R, Boing AN, Romijn FP, Hack CE, Sturk A. Cell-derived microparticles circulate in healthy humans and support low grade thrombin generation. Thromb Haemost 2001 Apr;85(4):639-46.

(87) Sinauridze El, Kireev DA, Popenko NY, Pichugin AV, Panteleev MA, Krymskaya OV, et al. Platelet microparticle membranes have 50- to 100-fold higher specific procoagulant activity than activated platelets. Thromb Haemost 2007 Mar;97(3):42534.

(88) Tesselaar ME, Romijn FP, van der Linden IK, Prins FA, Bertina RM, Osanto S. Microparticle-associated tissue factor activity: a link between cancer and thrombosis? J Thromb Haemost 2007 Mar;5(3):520-7.

(89) Hugel B, Socie G, Vu T, Toti F, Gluckman E, Freyssinet JM, et al. Elevated levels of circulating procoagulant microparticles in patients with paroxysmal nocturnal hemoglobinuria and aplastic anemia. Blood 1999 May 15;93(10):3451-6.

(90) Manno S, Takakuwa Y, Mohandas N. Identification of a functional role for lipid asymmetry in biological membranes: Phosphatidylserine-skeletal protein interactions modulate membrane stability. Proc Natl Acad Sci U S A 2002 Feb 19;99(4):1943-8.

(91) Bevers EM, Comfurius P, Dekkers DW, Zwaal RF. Lipid translocation across the plasma membrane of mammalian cells. Biochim Biophys Acta 1999 Aug 18;1439(3):317-30. 
(92) Freyssinet JM, Toti F. Formation of procoagulant microparticles and properties. Thromb Res 2010 Apr;125 Suppl 1:S46-S48.

(93) Schroit AJ, Zwaal RF. Transbilayer movement of phospholipids in red cell and platelet membranes. Biochim Biophys Acta 1991 Nov 13;1071(3):313-29.

(94) Morel O, Toti F, Jesel L, Freyssinet JM. Mechanisms of microparticle generation: on the trail of the mitochondrion! Semin Thromb Hemost 2010 Nov;36(8):833-44.

(95) van Helvoort A., Smith AJ, Sprong H, Fritzsche I, Schinkel AH, Borst P, et al. MDR1 P-glycoprotein is a lipid translocase of broad specificity, while MDR3 P-glycoprotein specifically translocates phosphatidylcholine. Cell 1996 Nov 1;87(3):507-17.

(96) Zwaal RF, Comfurius P, Bevers EM. Surface exposure of phosphatidylserine in pathological cells. Cell Mol Life Sci 2005 May;62(9):971-88.

(97) Rosing J, Bevers EM, Comfurius P, Hemker HC, van DG, Weiss HJ, et al. Impaired factor $\mathrm{X}$ and prothrombin activation associated with decreased phospholipid exposure in platelets from a patient with a bleeding disorder. Blood 1985 Jun;65(6):1557-61.

(98) Toti F, Satta N, Fressinaud E, Meyer D, Freyssinet JM. Scott syndrome, characterized by impaired transmembrane migration of procoagulant phosphatidylserine and hemorrhagic complications, is an inherited disorder. Blood 1996 Feb 15;87(4):1409-15.

(99) Henderson RM, Edwardson JM, Geisse NA, Saslowsky DE. Lipid rafts: feeling is believing. News Physiol Sci 2004 Apr;19:39-43.

(100) Hugel B, Martinez MC, Kunzelmann C, Freyssinet JM. Membrane microparticles: two sides of the coin. Physiology (Bethesda ) 2005 Feb;20:22-7.

(101) Varga-Szabo D, Braun A, Nieswandt B. Calcium signaling in platelets. J Thromb Haemost 2009 Jul;7(7):1057-66.

(102) MacKenzie A, Wilson HL, Kiss-Toth E, Dower SK, North RA, Surprenant A. Rapid secretion of interleukin-1beta by microvesicle shedding. Immunity 2001 Nov;15(5):825-35.

(103) Martinez MC, Martin S, Toti F, Fressinaud E, Dachary-Prigent J, Meyer D, et al. Significance of capacitative $\mathrm{Ca} 2+$ entry in the regulation of phosphatidylserine expression at the surface of stimulated cells. Biochemistry 1999 Aug 3;38(31):100928.

(104) Parekh AB, Putney JW, Jr. Store-operated calcium channels. Physiol Rev 2005 Apr;85(2):757-810.

(105) Kovacs T, Corvazier E, Papp B, Magnier C, Bredoux R, Enyedi A, et al. Controlled proteolysis of $\mathrm{Ca}(2+)$-ATPases in human platelet and non-muscle cell membrane vesicles. Evidence for a multi-sarco/endoplasmic reticulum $\mathrm{Ca}(2+)$-ATPase system. J Biol Chem 1994 Feb 25;269(8):6177-84.

(106) Gilio K, van KR, Braun A, Berna-Erro A, Feijge MA, Stegner D, et al. Roles of platelet STIM1 and Orai1 in glycoprotein VI- and thrombin-dependent procoagulant activity and thrombus formation. J Biol Chem 2010 Jul 30;285(31):23629-38.

(107) Cauwenberghs S, Feijge MA, Harper AG, Sage SO, Curvers J, Heemskerk JW. Shedding of procoagulant microparticles from unstimulated platelets by integrin- 
mediated destabilization of actin cytoskeleton. FEBS Lett 2006 Oct 2;580(22):531320.

(108) Fox JE, Reynolds CC, Austin CD. The role of calpain in stimulus-response coupling: evidence that calpain mediates agonist-induced expression of procoagulant activity in platelets. Blood 1990 Dec 15;76(12):2510-9.

(109) Sapet C, Simoncini S, Loriod B, Puthier D, Sampol J, Nguyen C, et al. Thrombininduced endothelial microparticle generation: identification of a novel pathway involving ROCK-II activation by caspase-2. Blood 2006 Sep 15;108(6):1868-76.

(110) Tramontano AF, O'Leary J, Black AD, Muniyappa R, Cutaia MV, El-Sherif N. Statin decreases endothelial microparticle release from human coronary artery endothelial cells: implication for the Rho-kinase pathway. Biochem Biophys Res Commun 2004 Jul 16;320(1):34-8.

(111) Curtis AM, Wilkinson PF, Gui M, Gales TL, Hu E, Edelberg JM. p38 mitogen-activated protein kinase targets the production of proinflammatory endothelial microparticles. J Thromb Haemost 2009 Apr;7(4):701-9.

(112) Obregon C, Rothen-Rutishauser B, Gitahi SK, Gehr P, Nicod LP. Exovesicles from human activated dendritic cells fuse with resting dendritic cells, allowing them to present alloantigens. Am J Pathol 2006 Dec;169(6):2127-36.

(113) Hashimoto K, Jayachandran M, Owen WG, Miller VM. Aggregation and microparticle production through toll-like receptor 4 activation in platelets from recently menopausal women. J Cardiovasc Pharmacol 2009 Jul;54(1):57-62.

(114) Elliott JI, Sardini A, Cooper JC, Alexander DR, Davanture S, Chimini G, et al. Phosphatidylserine exposure in B lymphocytes: a role for lipid packing. Blood 2006 Sep 1;108(5):1611-7.

(115) Raphael RM, Waugh RE, Svetina S, Zeks B. Fractional occurrence of defects in membranes and mechanically driven interleaflet phospholipid transport. Phys Rev E Stat Nonlin Soft Matter Phys 2001 Nov;64(5 Pt 1):051913.

(116) Bevers EM, Williamson PL. Phospholipid scramblase: an update. FEBS Lett 2010 Jul 2;584(13):2724-30.

(117) Yang MY, Chuang H, Chen RF, Yang KD. Reversible phosphatidylserine expression on blood granulocytes related to membrane perturbation but not DNA strand breaks. J Leukoc Biol 2002 Feb;71(2):231-7.

(118) Shcherbina A, Remold-O'Donnell E. Role of caspase in a subset of human platelet activation responses. Blood 1999 Jun 15;93(12):4222-31.

(119) Zhang H, Nimmer PM, Tahir SK, Chen J, Fryer RM, Hahn KR, et al. Bcl-2 family proteins are essential for platelet survival. Cell Death Differ 2007 May;14(5):943-51.

(120) Sebbagh M, Renvoize C, Hamelin J, Riche N, Bertoglio J, Breard J. Caspase-3mediated cleavage of ROCK I induces MLC phosphorylation and apoptotic membrane blebbing. Nat Cell Biol 2001 Apr;3(4):346-52.

(121) Babst M. A protein's final ESCRT. Traffic 2005 Jan;6(1):2-9.

(122) Michelet X, Djeddi A, Legouis R. Developmental and cellular functions of the ESCRT machinery in pluricellular organisms. Biol Cell 2010 Mar;102(3):191-202. 
(123) Tamai K, Tanaka N, Nakano T, Kakazu E, Kondo Y, Inoue J, et al. Exosome secretion of dendritic cells is regulated by Hrs, an ESCRT-0 protein. Biochem Biophys Res Commun 2010 Aug 27;399(3):384-90.

(124) Trajkovic K, Hsu C, Chiantia S, Rajendran L, Wenzel D, Wieland F, et al. Ceramide triggers budding of exosome vesicles into multivesicular endosomes. Science 2008 Feb 29;319(5867):1244-7.

(125) Möbius W, Ohno-Iwashita Y, van Donselaar EG, Oorschot VM, Shimada Y, Fujimoto $\mathrm{T}$, et al. Immunoelectron microscopic localization of cholesterol using biotinylated and non-cytolytic perfringolysin O. J Histochem Cytochem 2002 Jan;50(1):43-55.

(126) Matsuo H, Chevallier J, Mayran N, Le B, I, Ferguson C, Faure J, et al. Role of LBPA and Alix in multivesicular liposome formation and endosome organization. Science 2004 Jan 23;303(5657):531-4.

(127) White IJ, Bailey LM, Aghakhani MR, Moss SE, Futter CE. EGF stimulates annexin 1dependent inward vesiculation in a multivesicular endosome subpopulation. EMBO J 2006 Jan $11 ; 25(1): 1-12$.

(128) van NG, Charrin S, Simoes S, Romao M, Rochin L, Saftig P, et al. The tetraspanin CD63 regulates ESCRT-independent and -dependent endosomal sorting during melanogenesis. Dev Cell 2011 Oct 18;21(4):708-21.

(129) Buschow SI, Nolte-'t Hoen EN, van NG, Pols MS, ten BT, Lauwen M, et al. MHC II in dendritic cells is targeted to lysosomes or $\mathrm{T}$ cell-induced exosomes via distinct multivesicular body pathways. Traffic 2009 Oct;10(10):1528-42.

(130) Savina A, Fader CM, Damiani MT, Colombo MI. Rab11 promotes docking and fusion of multivesicular bodies in a calcium-dependent manner. Traffic 2005 Feb;6(2):13143.

(131) Ostrowski M, Carmo NB, Krumeich S, Fanget I, Raposo G, Savina A, et al. Rab27a and Rab27b control different steps of the exosome secretion pathway. Nat Cell Biol 2010 Jan;12(1):19-30.

(132) Hsu C, Morohashi Y, Yoshimura S, Manrique-Hoyos N, Jung S, Lauterbach MA, et al. Regulation of exosome secretion by Rab35 and its GTPase-activating proteins TBC1D10A-C. J Cell Biol 2010 Apr 19;189(2):223-32.

(133) Rao SK, Huynh C, Proux-Gillardeaux V, Galli T, Andrews NW. Identification of SNAREs involved in synaptotagmin VII-regulated lysosomal exocytosis. J Biol Chem 2004 May 7;279(19):20471-9.

(134) Lenassi M, Cagney G, Liao M, Vaupotic T, Bartholomeeusen K, Cheng Y, et al. HIV Nef is secreted in exosomes and triggers apoptosis in bystander CD4+ T cells. Traffic 2010 Jan;11(1):110-22.

(135) Fang Y, Wu N, Gan X, Yan W, Morrell JC, Gould SJ. Higher-order oligomerization targets plasma membrane proteins and HIV gag to exosomes. PLoS Biol 2007 Jun;5(6):e158.

(136) Booth AM, Fang Y, Fallon JK, Yang JM, Hildreth JE, Gould SJ. Exosomes and HIV Gag bud from endosome-like domains of the T cell plasma membrane. J Cell Biol 2006 Mar 13;172(6):923-35. 
(137) CHARGAFF E, WEST R. The biological significance of the thromboplastic protein of blood. J Biol Chem 1946 Nov;166(1):189-97.

(138) Warren BA, Vales $O$. The release of vesicles from platelets following adhesion to vessel walls in vitro. Br J Exp Pathol 1972 Apr;53(2):206-15.

(139) Polasek J, Richardson M, Moore MA, Blajchman MA. Evidence for an alternative mechanism of human platelet secretion involving peripheralization of secretory granules and formation of membrane-associated multivesicular structures. Thromb Res 1987 Mar 15;45(6):771-82.

(140) Aatonen M, Gronholm M, Siljander PR. Platelet-derived microvesicles: multitalented participants in intercellular communication. Semin Thromb Hemost 2012 Feb;38(1):102-13.

(141) Dasgupta SK, Argaiz ER, Mercado JE, Maul HO, Garza J, Enriquez AB, et al. Platelet senescence and phosphatidylserine exposure. Transfusion 2010 Oct;50(10):2167-75.

(142) Horstman LL, Ahn YS. Platelet microparticles: a wide-angle perspective. Crit Rev Oncol Hematol 1999 Apr;30(2):111-42.

(143) Barry OP, Pratico D, Savani RC, FitzGerald GA. Modulation of monocyte-endothelial cell interactions by platelet microparticles. J Clin Invest 1998 Jul 1;102(1):136-44.

(144) Barry OP, Kazanietz MG, Pratico D, FitzGerald GA. Arachidonic acid in platelet microparticles up-regulates cyclooxygenase-2-dependent prostaglandin formation via a protein kinase $\mathrm{C} /$ mitogen-activated protein kinase-dependent pathway. J Biol Chem 1999 Mar 12;274(11):7545-56.

(145) Jy W, Mao WW, Horstman L, Tao J, Ahn YS. Platelet microparticles bind, activate and aggregate neutrophils in vitro. Blood Cells Mol Dis 1995;21(3):217-31.

(146) Sadallah S, Eken C, Martin PJ, Schifferli JA. Microparticles (ectosomes) shed by stored human platelets downregulate macrophages and modify the development of dendritic cells. J Immunol 2011 Jun 1;186(11):6543-52.

(147) Sprague DL, Sowa JM, Elzey BD, Ratliff TL. The role of platelet CD154 in the modulation in adaptive immunity. Immunol Res 2007;39(1-3):185-93.

(148) Biro E, Nieuwland R, Tak PP, Pronk LM, Schaap MC, Sturk A, et al. Activated complement components and complement activator molecules on the surface of cellderived microparticles in patients with rheumatoid arthritis and healthy individuals. Ann Rheum Dis 2007 Aug;66(8):1085-92.

(149) Falanga A, Tartari CJ, Marchetti M. Microparticles in tumor progression. Thromb Res 2012 Apr;129 Suppl 1:S132-S136.

(150) Janowska-Wieczorek A, Majka M, Kijowski J, Baj-Krzyworzeka M, Reca R, Turner $A R$, et al. Platelet-derived microparticles bind to hematopoietic stem/progenitor cells and enhance their engraftment. Blood 2001 Nov 15;98(10):3143-9.

(151) Kim HK, Song KS, Chung JH, Lee KR, Lee SN. Platelet microparticles induce angiogenesis in vitro. Br J Haematol 2004 Feb;124(3):376-84.

(152) Ueba T, Nomura S, Inami N, Nishikawa T, Kajiwara M, Iwata R, et al. Plasma level of platelet-derived microparticles is associated with coronary heart disease risk score in healthy men. J Atheroscler Thromb 2010 Apr 30;17(4):342-9. 
(153) Nomura S, Uehata S, Saito S, Osumi K, Ozeki Y, Kimura Y. Enzyme immunoassay detection of platelet-derived microparticles and RANTES in acute coronary syndrome. Thromb Haemost 2003 Mar;89(3):506-12.

(154) Mallat Z, Benamer H, Hugel B, Benessiano J, Steg PG, Freyssinet JM, et al. Elevated levels of shed membrane microparticles with procoagulant potential in the peripheral circulating blood of patients with acute coronary syndromes. Circulation 2000 Feb 29;101(8):841-3.

(155) Bernal-Mizrachi L, Jy W, Jimenez JJ, Pastor J, Mauro LM, Horstman LL, et al. High levels of circulating endothelial microparticles in patients with acute coronary syndromes. Am Heart J 2003 Jun;145(6):962-70.

(156) van der Zee PM, Biro E, Ko Y, de Winter RJ, Hack CE, Sturk A, et al. P-selectin- and CD63-exposing platelet microparticles reflect platelet activation in peripheral arterial disease and myocardial infarction. Clin Chem 2006 Apr;52(4):657-64.

(157) Zeiger F, Stephan S, Hoheisel G, Pfeiffer D, Ruehlmann C, Koksch M. P-Selectin expression, platelet aggregates, and platelet-derived microparticle formation are increased in peripheral arterial disease. Blood Coagul Fibrinolysis 2000 Dec;11(8):723-8.

(158) Preston RA, Jy W, Jimenez JJ, Mauro LM, Horstman LL, Valle M, et al. Effects of severe hypertension on endothelial and platelet microparticles. Hypertension 2003 Feb;41(2):211-7.

(159) Diehl P, Aleker M, Helbing T, Sossong V, Germann M, Sorichter S, et al. Increased platelet, leukocyte and endothelial microparticles predict enhanced coagulation and vascular inflammation in pulmonary hypertension. J Thromb Thrombolysis 2011 Feb;31(2):173-9.

(160) Bal L, Ederhy S, Di AE, Toti F, Zobairi F, Dufaitre G, et al. Factors influencing the level of circulating procoagulant microparticles in acute pulmonary embolism. Arch Cardiovasc Dis 2010 Jun;103(6-7):394-403.

(161) Azzam H, Zagloul M. Elevated platelet microparticle levels in valvular atrial fibrillation. Hematology 2009 Dec;14(6):357-60.

(162) Rectenwald JE, Myers DD, Jr., Hawley AE, Longo C, Henke PK, Guire KE, et al. Ddimer, P-selectin, and microparticles: novel markers to predict deep venous thrombosis. A pilot study. Thromb Haemost 2005 Dec;94(6):1312-7.

(163) Lee YJ, Jy W, Horstman LL, Janania J, Reyes Y, Kelley RE, et al. Elevated platelet microparticles in transient ischemic attacks, lacunar infarcts, and multiinfarct dementias. Thromb Res 1993 Nov 15;72(4):295-304.

(164) Lukasik M, Rozalski M, Luzak B, Michalak M, Ambrosius W, Watala C, et al. Enhanced platelet-derived microparticle formation is associated with carotid atherosclerosis in convalescent stroke patients. Platelets 2013;24(1):63-70.

(165) Lackner P, Dietmann A, Beer R, Fischer M, Broessner G, Helbok R, et al. Cellular microparticles as a marker for cerebral vasospasm in spontaneous subarachnoid hemorrhage. Stroke 2010 Oct;41(10):2353-7.

(166) Tantawy AA, Matter RM, Hamed AA, Shams El Din El Telbany MA. Platelet microparticles in immune thrombocytopenic purpura in pediatrics. Pediatr Hematol Oncol 2010 May;27(4):283-96. 
(167) Galli M, Grassi A, Barbui T. Platelet-derived microvesicles in thrombotic thrombocytopenic purpura and hemolytic uremic syndrome. Thromb Haemost 1996 Mar;75(3):427-31.

(168) Sewify EM, Sayed D, Abdel AAI RF, Ahmad HM, Abdou MA. Increased circulating red cell microparticles (RMP) and platelet microparticles (PMP) in immune thrombocytopenic purpura. Thrombosis Research 2013 Feb;131(2):e59-e63.

(169) Wiedmer T, Hall SE, Ortel TL, Kane WH, Rosse WF, Sims PJ. Complement-induced vesiculation and exposure of membrane prothrombinase sites in platelets of paroxysmal nocturnal hemoglobinuria. Blood 1993 Aug 15;82(4):1192-6.

(170) Warkentin TE, Hayward CP, Boshkov LK, Santos AV, Sheppard JA, Bode AP, et al. Sera from patients with heparin-induced thrombocytopenia generate platelet-derived microparticles with procoagulant activity: an explanation for the thrombotic complications of heparin-induced thrombocytopenia. Blood 1994 Dec 1;84(11):36919.

(171) Hughes M, Hayward CP, Warkentin TE, Horsewood P, Chorneyko KA, Kelton JG. Morphological analysis of microparticle generation in heparin-induced thrombocytopenia. Blood 2000 Jul 1;96(1):188-94.

(172) Gemmell $\mathrm{CH}$, Sefton MV, Yeo EL. Platelet-derived microparticle formation involves glycoprotein Ilb-IIla. Inhibition by RGDS and a Glanzmann's thrombasthenia defect. J Biol Chem 1993 Jul 15;268(20):14586-9.

(173) Sims PJ, Wiedmer T, Esmon CT, Weiss HJ, Shattil SJ. Assembly of the platelet prothrombinase complex is linked to vesiculation of the platelet plasma membrane. Studies in Scott syndrome: an isolated defect in platelet procoagulant activity. J Biol Chem 1989 Oct 15;264(29):17049-57.

(174) Stormorken H, Holmsen H, Sund R, Sakariassen KS, Hovig T, Jellum E, et al. Studies on the haemostatic defect in a complicated syndrome. An inverse Scott syndrome platelet membrane abnormality? Thromb Haemost 1995 Nov;74(5):1244-51.

(175) Shet AS, Aras O, Gupta K, Hass MJ, Rausch DJ, Saba N, et al. Sickle blood contains tissue factor-positive microparticles derived from endothelial cells and monocytes. Blood 2003 Oct 1;102(7):2678-83.

(176) Tomer A, Harker LA, Kasey S, Eckman JR. Thrombogenesis in sickle cell disease. J Lab Clin Med 2001 Jun;137(6):398-407.

(177) Kim HK, Song KS, Park YS, Kang YH, Lee YJ, Lee KR, et al. Elevated levels of circulating platelet microparticles, VEGF, IL-6 and RANTES in patients with gastric cancer: possible role of a metastasis predictor. Eur J Cancer 2003 Jan;39(2):184-91.

(178) Janowska-Wieczorek A, Wysoczynski M, Kijowski J, Marquez-Curtis L, Machalinski B, Ratajczak J, et al. Microvesicles derived from activated platelets induce metastasis and angiogenesis in lung cancer. Int J Cancer 2005 Feb 20;113(5):752-60.

(179) Janowska-Wieczorek A, Marquez-Curtis LA, Wysoczynski M, Ratajczak MZ. Enhancing effect of platelet-derived microvesicles on the invasive potential of breast cancer cells. Transfusion 2006 Jul;46(7):1199-209.

(180) Toth B, Liebhardt S, Steinig K, Ditsch N, Rank A, Bauerfeind I, et al. Platelet-derived microparticles and coagulation activation in breast cancer patients. Thromb Haemost 2008 Oct;100(4):663-9. 
(181) Dashevsky O, Varon D, Brill A. Platelet-derived microparticles promote invasiveness of prostate cancer cells via upregulation of MMP-2 production. Int J Cancer 2009 Apr 15;124(8):1773-7.

(182) Helley D, Banu E, Bouziane A, Banu A, Scotte F, Fischer AM, et al. Platelet microparticles: a potential predictive factor of survival in hormone-refractory prostate cancer patients treated with docetaxel-based chemotherapy. Eur Urol 2009 Sep;56(3):479-84.

(183) Mostefai HA, Meziani F, Mastronardi ML, Agouni A, Heymes C, Sargentini C, et al. Circulating microparticles from patients with septic shock exert protective role in vascular function. Am J Respir Crit Care Med 2008 Dec 1;178(11):1148-55.

(184) Soriano AO, Jy W, Chirinos JA, Valdivia MA, Velasquez HS, Jimenez JJ, et al. Levels of endothelial and platelet microparticles and their interactions with leukocytes negatively correlate with organ dysfunction and predict mortality in severe sepsis. Crit Care Med 2005 Nov;33(11):2540-6.

(185) Robertson C, Booth SA, Beniac DR, Coulthart MB, Booth TF, McNicol A. Cellular prion protein is released on exosomes from activated platelets. Blood 2006 May 15;107(10):3907-11.

(186) Pereira J, Alfaro G, Goycoolea M, Quiroga T, Ocqueteau M, Massardo L, et al. Circulating platelet-derived microparticles in systemic lupus erythematosus. Association with increased thrombin generation and procoagulant state. Thromb Haemost 2006 Jan;95(1):94-9.

(187) Sellam J, Proulle V, Jungel A, Ittah M, Miceli RC, Gottenberg JE, et al. Increased levels of circulating microparticles in primary Sjogren's syndrome, systemic lupus erythematosus and rheumatoid arthritis and relation with disease activity. Arthritis Res Ther 2009;11(5):R156.

(188) Jy W, Tiede M, Bidot CJ, Horstman LL, Jimenez JJ, Chirinos J, et al. Platelet activation rather than endothelial injury identifies risk of thrombosis in subjects positive for antiphospholipid antibodies. Thromb Res 2007;121(3):319-25.

(189) Guiducci S, Distler JH, Jungel A, Huscher D, Huber LC, Michel BA, et al. The relationship between plasma microparticles and disease manifestations in patients with systemic sclerosis. Arthritis Rheum 2008 Sep;58(9):2845-53.

(190) Nomura S, Inami N, Ozaki Y, Kagawa H, Fukuhara S. Significance of microparticles in progressive systemic sclerosis with interstitial pneumonia. Platelets 2008 May;19(3):192-8.

(191) Boilard E, Nigrovic PA, Larabee K, Watts GF, Coblyn JS, Weinblatt ME, et al. Platelets amplify inflammation in arthritis via collagen-dependent microparticle production. Science 2010 Jan 29;327(5965):580-3.

(192) Knijff-Dutmer EA, Koerts J, Nieuwland R, Kalsbeek-Batenburg EM, van de Laar MA. Elevated levels of platelet microparticles are associated with disease activity in rheumatoid arthritis. Arthritis Rheum 2002 Jun;46(6):1498-503.

(193) Daniel L, Fakhouri F, Joly D, Mouthon L, Nusbaum P, Grunfeld JP, et al. Increase of circulating neutrophil and platelet microparticles during acute vasculitis and hemodialysis. Kidney Int 2006 Apr;69(8):1416-23. 
(194) Sabatier F, Darmon P, Hugel B, Combes V, Sanmarco M, Velut JG, et al. Type 1 and type 2 diabetic patients display different patterns of cellular microparticles. Diabetes 2002 Sep;51(9):2840-5.

(195) Sheremata WA, Jy W, Horstman LL, Ahn YS, Alexander JS, Minagar A. Evidence of platelet activation in multiple sclerosis. J Neuroinflammation 2008;5:27.

(196) Diamant M, Nieuwland R, Pablo RF, Sturk A, Smit JW, Radder JK. Elevated numbers of tissue-factor exposing microparticles correlate with components of the metabolic syndrome in uncomplicated type 2 diabetes mellitus. Circulation 2002 Nov 5;106(19):2442-7.

(197) Tan KT, Tayebjee MH, Lim HS, Lip GY. Clinically apparent atherosclerotic disease in diabetes is associated with an increase in platelet microparticle levels. Diabet Med 2005 Dec;22(12):1657-62.

(198) Koga H, Sugiyama S, Kugiyama K, Fukushima H, Watanabe K, Sakamoto T, et al. Elevated levels of remnant lipoproteins are associated with plasma platelet microparticles in patients with type-2 diabetes mellitus without obstructive coronary artery disease. Eur Heart J 2006 Apr;27(7):817-23.

(199) Shouzu A, Nomura S, Omoto S, Hayakawa T, Nishikawa M, Iwasaka T. Effect of ticlopidine on monocyte-derived microparticles and activated platelet markers in diabetes mellitus. Clin Appl Thromb Hemost 2004 Apr;10(2):167-73.

(200) Agouni A, Lagrue-Lak-Hal AH, Ducluzeau PH, Mostefai HA, Draunet-Busson C, Leftheriotis $\mathrm{G}$, et al. Endothelial dysfunction caused by circulating microparticles from patients with metabolic syndrome. Am J Pathol 2008 Oct;173(4):1210-9.

(201) Murakami T, Horigome H, Tanaka K, Nakata Y, Katayama Y, Matsui A. Effects of diet with or without exercise on leptin and anticoagulation proteins levels in obesity. Blood Coagul Fibrinolysis 2007 Jul;18(5):389-94.

(202) Ayers L, Ferry B, Craig S, Nicoll D, Stradling JR, Kohler M. Circulating cell-derived microparticles in patients with minimally symptomatic obstructive sleep apnoea. Eur Respir J 2009 Mar;33(3):574-80.

(203) Ando M, Iwata A, Ozeki Y, Tsuchiya K, Akiba T, Nihei H. Circulating platelet-derived microparticles with procoagulant activity may be a potential cause of thrombosis in uremic patients. Kidney Int 2002 Nov;62(5):1757-63.

(204) Faille D, Combes V, Mitchell AJ, Fontaine A, Juhan-Vague I, Alessi MC, et al. Platelet microparticles: a new player in malaria parasite cytoadherence to human brain endothelium. FASEB J 2009 Oct;23(10):3449-58.

(205) Thom SR, Yang M, Bhopale VM, Huang S, Milovanova TN. Microparticles initiate decompression-induced neutrophil activation and subsequent vascular injuries. J Appl Physiol 2011 Feb;110(2):340-51.

(206) Lok CA, Nieuwland R, Sturk A, Hau CM, Boer K, Vanbavel E, et al. Microparticleassociated P-selectin reflects platelet activation in preeclampsia. Platelets 2007 Feb;18(1):68-72.

(207) Lok CA, Van Der Post JA, Sargent IL, Hau CM, Sturk A, Boer K, et al. Changes in microparticle numbers and cellular origin during pregnancy and preeclampsia. Hypertens Pregnancy 2008;27(4):344-60. 
(208) VanWijk MJ, Nieuwland R, Boer K, Van Der Post JA, Vanbavel E, Sturk A. Microparticle subpopulations are increased in preeclampsia: possible involvement in vascular dysfunction? Am J Obstet Gynecol 2002 Aug;187(2):450-6.

(209) Kaptan K, Beyan C, Ifran A, Pekel A. Platelet-derived microparticle levels in women with recurrent spontaneous abortion. Int J Gynaecol Obstet 2008 Sep;102(3):271-4.

(210) Matsubara E, Shoji M, Murakami T, Abe K, Frangione B, Ghiso J. Platelet microparticles as carriers of soluble Alzheimer's amyloid beta (sAbeta). Ann N Y Acad Sci 2002 Nov;977:340-8.

(211) Sevush S, Jy W, Horstman LL, Mao WW, Kolodny L, Ahn YS. Platelet activation in Alzheimer disease. Arch Neurol 1998 Apr;55(4):530-6.

(212) Oyabu C, Morinobu A, Sugiyama D, Saegusa J, Tanaka S, Morinobu S, et al. Plasma platelet-derived microparticles in patients with connective tissue diseases. J Rheumatol $2011 \mathrm{Apr} ; 38(4): 680-4$.

(213) Siljander PR. Platelet-derived microparticles - an updated perspective. Thromb Res 2011 Jan;127 Suppl 2:S30-S33.

(214) Chandler WL, Yeung W, Tait JF. A new microparticle size calibration standard for use in measuring smaller microparticles using a new flow cytometer. J Thromb Haemost 2011 Jun;9(6):1216-24.

(215) Cramer EM, Norol F, Guichard J, Breton-Gorius J, Vainchenker W, Masse JM, et al. Ultrastructure of platelet formation by human megakaryocytes cultured with the Mpl ligand. Blood 1997 Apr 1;89(7):2336-46.

(216) Rank A, Nieuwland R, Delker R, Pihusch V, Wilkowski R, Toth B, et al. Surveillance of megakaryocytic function by measurement of CD61-exposing microparticles in allogeneic hematopoietic stem cell recipients. Clin Transplant 2011 May;25(3):E233E242.

(217) Toth B, Nikolajek K, Rank A, Nieuwland R, Lohse P, Pihusch V, et al. Gender-specific and menstrual cycle dependent differences in circulating microparticles. Platelets 2007 Nov;18(7):515-21.

(218) Robert S, Poncelet P, Lacroix R, Arnaud L, Giraudo L, Hauchard A, et al. Standardization of platelet-derived microparticle counting using calibrated beads and a Cytomics FC500 routine flow cytometer: a first step towards multicenter studies? J Thromb Haemost 2009 Jan;7(1):190-7.

(219) Bretelle F, Sabatier F, Desprez D, Camoin L, Grunebaum L, Combes V, et al. Circulating microparticles: a marker of procoagulant state in normal pregnancy and pregnancy complicated by preeclampsia or intrauterine growth restriction. Thromb Haemost 2003 Mar;89(3):486-92.

(220) Chaar V, Romana M, Tripette J, Broquere C, Huisse MG, Hue O, et al. Effect of strenuous physical exercise on circulating cell-derived microparticles. Clin Hemorheol Microcirc 2011;47(1):15-25.

(221) Sossdorf M, Otto GP, Claus RA, Gabriel HH, Losche W. Cell-derived microparticles promote coagulation after moderate exercise. Med Sci Sports Exerc 2011 Jul;43(7):1169-76. 
(222) Rank A, Nieuwland R, Nikolajek K, Rosner S, Wallwiener LM, Hiller E, et al. Hormone replacement therapy leads to increased plasma levels of platelet derived microparticles in postmenopausal women. Arch Gynecol Obstet 2012 Apr;285(4):1035-41.

(223) Rank A, Nieuwland R, Roesner S, Nikolajek K, Hiller E, Toth B. Climacteric lowers plasma levels of platelet-derived microparticles: a pilot study in pre- versus postmenopausal women. Acta Haematol 2012;128(1):53-9.

(224) Krailadsiri P, Seghatchian J. Are all leucodepleted platelet concentrates equivalent? Comparison of Cobe LRS Turbo, Haemonetics MCS+ LD, and filtered pooled buffycoat-derived platelets. Vox Sang 2000;78(3):171-5.

(225) Chang CP, Zhao J, Wiedmer T, Sims PJ. Contribution of platelet microparticle formation and granule secretion to the transmembrane migration of phosphatidylserine. J Biol Chem 1993 Apr 5;268(10):7171-8.

(226) Flaumenhaft R, Dilks JR, Richardson J, Alden E, Patel-Hett SR, Battinelli E, et al. Megakaryocyte-derived microparticles: direct visualization and distinction from platelet-derived microparticles. Blood 2009 Jan 29;113(5):1112-21.

(227) Italiano JE, Jr., Mairuhu AT, Flaumenhaft R. Clinical relevance of microparticles from platelets and megakaryocytes. Curr Opin Hematol 2010 Nov;17(6):578-84.

(228) Rank A, Nieuwland R, Crispin A, Grutzner S, Iberer M, Toth B, et al. Clearance of platelet microparticles in vivo. Platelets 2011;22(2):111-6.

(229) Wiedmer T, Shattil SJ, Cunningham M, Sims PJ. Role of calcium and calpain in complement-induced vesiculation of the platelet plasma membrane and in the exposure of the platelet factor Va receptor. Biochemistry 1990 Jan 23;29(3):623-32.

(230) Miyazaki Y, Nomura S, Miyake T, Kagawa H, Kitada C, Taniguchi H, et al. High shear stress can initiate both platelet aggregation and shedding of procoagulant containing microparticles. Blood 1996 Nov 1;88(9):3456-64.

(231) Chow TW, Hellums JD, Thiagarajan P. Thrombin receptor activating peptide (SFLLRN) potentiates shear-induced platelet microvesiculation. J Lab Clin Med 2000 Jan;135(1):66-72.

(232) Sakariassen KS, Holme PA, Orvim U, Barstad RM, Solum NO, Brosstad FR. Shearinduced platelet activation and platelet microparticle formation in native human blood. Thromb Res 1998 Dec 15;92(6 Suppl 2):S33-S41.

(233) Holme PA, Orvim U, Hamers MJ, Solum NO, Brosstad FR, Barstad RM, et al. Shearinduced platelet activation and platelet microparticle formation at blood flow conditions as in arteries with a severe stenosis. Arterioscler Thromb Vasc Biol 1997 Apr;17(4):646-53.

(234) Gemmell CH, Ramirez SM, Yeo EL, Sefton MV. Platelet activation in whole blood by artificial surfaces: identification of platelet-derived microparticles and activated platelet binding to leukocytes as material-induced activation events. J Lab Clin Med 1995 Feb;125(2):276-87.

(235) Bode AP, Knupp CL. Effect of cold storage on platelet glycoprotein lb and vesiculation. Transfusion 1994 Aug;34(8):690-6. 
(236) Siljander P, Carpen O, Lassila R. Platelet-derived microparticles associate with fibrin during thrombosis. Blood 1996 Jun 1;87(11):4651-63.

(237) Rodman NF, Jr., Painter JC, McDEVITT NB. Platelet disintegration during clotting. J Cell Biol 1963 Feb;16:225-41.

(238) Dale GL, Friese P. Bax activators potentiate coated-platelet formation. J Thromb Haemost 2006 Dec;4(12):2664-9.

(239) Rand ML, Wang H, Bang KW, Packham MA, Freedman J. Rapid clearance of procoagulant platelet-derived microparticles from the circulation of rabbits. J Thromb Haemost 2006 Jul;4(7):1621-3.

(240) Ravichandran KS. Beginnings of a good apoptotic meal: the find-me and eat-me signaling pathways. Immunity 2011 Oct 28;35(4):445-55.

(241) Lemke G, Burstyn-Cohen T. TAM receptors and the clearance of apoptotic cells. Ann N Y Acad Sci 2010 Oct;1209:23-9.

(242) Dasgupta SK, Abdel-Monem H, Niravath P, Le A, Bellera RV, Langlois K, et al. Lactadherin and clearance of platelet-derived microvesicles. Blood 2009 Feb $5 ; 113(6): 1332-9$.

(243) Fontana V, Jy W, Ahn ER, Dudkiewicz P, Horstman LL, Duncan R, et al. Increased procoagulant cell-derived microparticles (C-MP) in splenectomized patients with ITP. Thrombosis Research 2008;122(5):599-603.

(244) Pisetsky DS. Platelet microparticles: making blood a bad humor. J Rheumatol 2011 Apr;38(4):590-2.

(245) Lacroix R, Robert S, Poncelet P, Kasthuri RS, Key NS, Dignat-George F. Standardization of platelet-derived microparticle enumeration by flow cytometry with calibrated beads: results of the International Society on Thrombosis and Haemostasis SSC Collaborative workshop. J Thromb Haemost 2010 Nov;8(11):2571-4.

(246) Robert S, Lacroix R, Poncelet P, Harhouri K, Bouriche T, Judicone C, et al. Highsensitivity flow cytometry provides access to standardized measurement of small-size microparticles--brief report. Arterioscler Thromb Vasc Biol 2012 Apr;32(4):1054-8.

(247) Raposo G, Stoorvogel W. Extracellular vesicles: Exosomes, microvesicles, and friends. The Journal of Cell Biology 2013 Feb 18;200(4):373-83.

(248) Dragovic RA, Gardiner C, Brooks AS, Tannetta DS, Ferguson DJ, Hole P, et al. Sizing and phenotyping of cellular vesicles using Nanoparticle Tracking Analysis. Nanomedicine 2011 Dec;7(6):780-8.

(249) van der Pol E, Hoekstra AG, Sturk A, Otto C, van Leeuwen TG, Nieuwland R. Optical and non-optical methods for detection and characterization of microparticles and exosomes. J Thromb Haemost 2010 Dec;8(12):2596-607.

(250) Zwicker JI, Lacroix R, Dignat-George F, Furie BC, Furie B. Measurement of platelet microparticles. Methods Mol Biol 2012;788:127-39.

(251) Lacroix R, Judicone C, Poncelet P, Robert S, Arnaud L, Sampol J, et al. Impact of pre-analytical parameters on the measurement of circulating microparticles: towards standardization of protocol. J Thromb Haemost 2012 Mar;10(3):437-46. 
(252) Lacroix R, Robert S, Poncelet P, Dignat-George F. Overcoming limitations of microparticle measurement by flow cytometry. Semin Thromb Hemost 2010 Nov;36(8):807-18.

(253) Shah MD, Bergeron AL, Dong JF, Lopez JA. Flow cytometric measurement of microparticles: pitfalls and protocol modifications. Platelets 2008 Aug;19(5):365-72.

(254) Freyssinet JM, Toti F. Membrane microparticle determination: at least seeing what's being sized! J Thromb Haemost 2010 Feb;8(2):311-4.

(255) Harrison P, Gardiner C. Invisible vesicles swarm within the iceberg. J Thromb Haemost 2012 May;10(5):916-8.

(256) Montoro-Garcia S, Shantsila E, Orenes-Pinero E, Lozano ML, Lip GY. An innovative flow cytometric approach for small-size platelet microparticles: influence of calcium. Thromb Haemost 2012 Aug;108(2):373-83.

(257) Nollet KE, Saito S, Ono T, Ngoma A, Ohto H. Microparticle formation in apheresis platelets is not affected by three leukoreduction filters. Transfusion 2013 Jan 24.

(258) Cookson P, Sutherland J, Turner C, Bashir S, Wiltshire M, Hancock V, et al. Platelet apoptosis and activation in platelet concentrates stored for up to 12 days in plasma or additive solution. Transfus Med 2010 Dec;20(6):392-402.

(259) Gardiner C, Ferreira YJ, Dragovic RA, Redman CWG, Sargent IL. Extracellular vesicle sizing and enumeration by nanoparticle tracking analysis. Journal of Extracellular Vesicles; Vol 2 (2013) 2013 Feb 15.

(260) Hoen EN, van der Vlist EJ, Aalberts M, Mertens HC, Bosch BJ, Bartelink W, et al. Quantitative and qualitative flow cytometric analysis of nanosized cell-derived membrane vesicles. Nanomedicine 2012 Jul;8(5):712-20.

(261) Pienimaeki-Roemer A, Ruebsaamen K, Boettcher A, Orso E, Scherer M, Liebisch G, et al. Stored platelets alter glycerophospholipid and sphingolipid species, which are differentially transferred to newly released extracellular vesicles. Transfusion $2012 \mathrm{Jul}$ 15.

(262) NanoSight. NanoSight NTA, Operating Manual. 2012.

(263) Henseler O, Heiden M, Haschberger B. Bericht zur Meldung nach §21 TFG für das Jahr 2007. Paul-Ehrlich-Institut; Bundesamt für Sera und Impfstoffe; 2013.

(264) Henseler O. PEI Meldung Blutprodukte TFG-21. 2013.

(265) Slichter SJ, Bolgiano D, Jones MK, Christoffel T, Corson J, Rose L, et al. Viability and function of 8-day-stored apheresis platelets. Transfusion 2006 Oct;46(10):1763-9.

(266) Anderson KC, Lew MA, Gorgone BC, Martel J, Leamy CB, Sullivan B. Transfusionrelated sepsis after prolonged platelet storage. Am J Med 1986 Sep;81(3):405-11.

(267) Witwer KW, Buz+ís El, Bemis LT, Bora A, L+ñsser C, L+Âtvall J, et al. Standardization of sample collection, isolation and analysis methods in extracellular vesicle research. Journal of Extracellular Vesicles; Vol 2 (2013) incl Supplements 2013 May 27. 
(268) Weiss EJ, Goldschmidt-Clermont PJ, Grigoryev D, Jin Y, Kickler TS, Bray PF. A monoclonal antibody (SZ21) specific for platelet GPIIla distinguishes P1A1 from P1A2. Tissue Antigens 1995 Nov;46(5):374-81.

(269) Thon JN, Devine DV. Translation of glycoprotein Illa in stored blood platelets. Transfusion 2007 Dec;47(12):2260-70.

(270) Thon JN, Schubert P, Devine DV. Platelet storage lesion: a new understanding from a proteomic perspective. Transfus Med Rev 2008 Oct;22(4):268-79.

(271) Han J, Lim CJ, Watanabe N, Soriani A, Ratnikov B, Calderwood DA, et al. Reconstructing and deconstructing agonist-induced activation of integrin alphallbbeta3. Curr Biol 2006 Sep 19;16(18):1796-806.

(272) Schubert P, Devine DV. Proteomics meets blood banking: identification of protein targets for the improvement of platelet quality. J Proteomics 2010 Jan 3;73(3):436-44.

(273) Schubert P, Culibrk B, Karwal S, Slichter SJ, Devine DV. Optimization of platelet concentrate quality: application of proteomic technologies to donor management. $J$ Proteomics 2012 Dec 5;76 Spec No.:329-36.

(274) Vasconcelos E, Figueiredo AC, Seghatchian J. Quality of platelet concentrates derived by platelet rich plasma, buffy coat and Apheresis. Transfus Apher Sci 2003 Aug;29(1):13-6.

(275) Garner SF, Jones Cl, Stephens J, Burns P, Walton J, Bernard A, et al. Apheresis donors and platelet function: inherent platelet responsiveness influences platelet quality. Transfusion $2008 \mathrm{Apr} ; 48(4): 673-80$.

(276) Flaumenhaft R, Mairuhu AT, Italiano JE. Platelet- and megakaryocyte-derived microparticles. Semin Thromb Hemost 2010 Nov;36(8):881-7.

(277) Hagberg IA, Akkok CA, Lyberg T, Kjeldsen-Kragh J. Apheresis-induced platelet activation:comparison of three types of cell separators. Transfusion 2000 Feb;40(2):182-92.

(278) Dumont LJ, Vandenbroeke T, Ault KA. Platelet surface P-selectin measurements in platelet preparations: an international collaborative study. Biomedical Excellence for Safer Transfusion (BEST) Working Party of the International Society of Blood Transfusion (ISBT). Transfus Med Rev 1999 Jan;13(1):31-42.

(279) Dumont LJ, Vandenbroeke T. Seven-day storage of apheresis platelets: report of an in vitro study. Transfusion 2003 Feb;43(2):143-50.

(280) Sweeney JD, Holme S, Moroff G. Storage of apheresis platelets after gamma radiation. Transfusion 1994 Sep;34(9):779-83.

(281) Sweeney JD, Holme S, Heaton A. Quality of platelet concentrates. Immunol Invest 1995 Jan;24(1-2):353-70.

(282) Siedlecki CA, Wang IW, Higashi JM, Kottke-Marchant K, Marchant RE. Plateletderived microparticles on synthetic surfaces observed by atomic force microscopy and fluorescence microscopy. Biomaterials 1999 Aug;20(16):1521-9.

(283) Vickers KC, Palmisano BT, Shoucri BM, Shamburek RD, Remaley AT. MicroRNAs are transported in plasma and delivered to recipient cells by high-density lipoproteins. Nat Cell Biol 2011 Apr;13(4):423-33. 
(284) Federici AB, Vanelli C, Arrigoni L. Transfusion issues in cancer patients. Thromb Res 2012 Apr;129 Suppl 1:S60-S65.

(285) Slichter SJ. Platelet transfusion therapy. Hematol Oncol Clin North Am 2007 Aug;21(4):697-729, vii.

(286) Garcia BA, Smalley DM, Cho H, Shabanowitz J, Ley K, Hunt DF. The platelet microparticle proteome. J Proteome Res 2005 Sep;4(5):1516-21.

(287) Rock G, Adams GA, Labow RS. The effects of irradiation on platelet function. Transfusion 1988 Sep;28(5):451-5.

(288) Wysoczynski M, Ratajczak MZ. Lung cancer secreted microvesicles: underappreciated modulators of microenvironment in expanding tumors. Int $\mathrm{J}$ Cancer 2009 Oct 1;125(7):1595-603.

(289) Shattil SJ, Ginsberg MH, Brugge JS. Adhesive signaling in platelets. Curr Opin Cell Biol 1994 Oct;6(5):695-704.

(290) Garcia BA, Smalley DM, Cho H, Shabanowitz J, Ley K, Hunt DF. The platelet microparticle proteome. J Proteome Res 2005 Sep;4(5):1516-21.

(291) Labrie A, Marshall A, Bedi H, Maurer-Spurej E. Characterization of platelet concentrates using dynamic light scattering. Transfus Med Hemother 2013 Apr;40(2):93-100. 